\title{
Active cancellation of 3D Tollmien-Schlichting waves in the presence of sound and vibrations
}

\author{
Dissertation \\ zur Erlangung des Doktorgrades \\ der Mathematisch-Naturwissenschaftlichen Fakultäten \\ der Georg-August-Universität Göttingen
}

vorgelegt von

Holger Opfer

aus

Bad Hersfeld

Göttingen 2002 
D 7

Referent: Prof. Dr. D. Ronneberger

Korreferent: Prof. Dr. H.Eckelmann

Tag der mündlichen Prüfung: 19.9.2002 


\section{Contents}

1 Introduction 1

1.1 The relevance of the subject . . . . . . . . . . . . . . 1

1.2 Previous experimental studies in the field . . . . . . . . . . . . . 2

1.3 The objectives of this investigation . . . . . . . . . . . . . 4

2 Dynamics of the boundary layer 5

2.1 Receptivity ........................... 5

2.2 Linear instability . . . . . . . . . . . . . . . 6

2.3 Dispersion characteristics of oblique waves . . . . . . . . . . . 9

3 Demands on the active wave cancellation system 13

3.1 The influence of the receptivity and the dispersion characteristics on the design of a wave cancellation system . . . . . . . . . . . . . . 14

3.2 The optimum linear model of the system . . . . . . . . . . . . . . 18

3.3 The observability and controllability of the disturbance wave field . . . 21

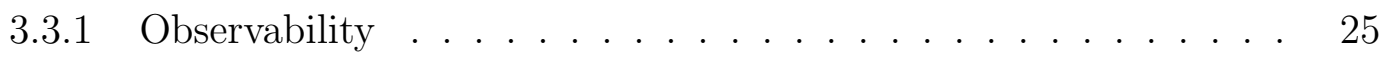

3.3.2 Controllability . . . . . . . . . . . . . . . 25

3.4 The constraint of causality . . . . . . . . . . . . . . . 26

3.5 Consideration of the dispersion characteristics . . . . . . . . . . 27

3.6 The real-time constraint to the controller . . . . . . . . . . . . . 27

4 Adaptive signal processing methods $\quad 29$

4.1 Adaptive filters . . . . . . . . . . . . . . . . . . . . . . . . . . 29

4.1 .1 Digital filters . . . . . . . . . . . . . . . . . 29 
4.1.2 Least-mean-square (LMS) algorithm . . . . . . . . . . . . . . 30

4.2 Active wave cancellation $(\mathrm{AWC}) \ldots \ldots . \ldots . \ldots . \ldots 34$

4.2 .1 System identification . . . . . . . . . . . . . . . 34

4.2.2 Single-input-single-output (SISO) system . . . . . . . . . . . 35

4.2.3 Multiple-input-multiple-output (MIMO) system . . . . . . . . . 38

4.2.4 Convergence and stability of the MIMO-filtered-X-LMS . . . . . 42

$5 \quad$ Experimental set-up $\quad 45$

5.1 The wind tunnel and the experimental equipment . . . . . . . . . 46

5.2 Hot-wire measurements . . . . . . . . . . . . . . . . . . 48

5.3 The real-time signal processing unit . . . . . . . . . . . . . . 49

5.4 Digital reduction of uncorrelated electric contamination of the signals . 51

6 Development of the active wave cancellation device 53

6.1 Preliminary cancellation device (Type I) . . . . . . . . . . . . . 53

6.2 Improved cancellation device (Type II) . . . . . . . . . . . . . . 62

7 Disturbance development in the boundary layer $\quad 69$

7.1 Blasius condition of the boundary layer . . . . . . . . . . . . . . 69

7.2 Point source experiment . . . . . . . . . . . . . . . . 73

8 Active wave cancellation experiments $\quad 89$

8.1 Application of the MIMO-AWC system to naturally occurring disturbance 89

8.2 The spatial spectrum of naturally occurring disturbances . . . . . . . 95

8.3 The application of the MIMO AWC system to broadband point-source disturbances . . . . . . . . . . . . . . . . . . 99 99

9 Acoustic and vibrational influences on the control system 103

9.1 Modification of the experimental set-up . . . . . . . . . . . . . . . 103

9.2 SISO AWC system in a noisy environment . . . . . . . . . . . . . . . . 104

9.3 Acoustic feedback effects . . . . . . . . . . . . . . . . . . . . . . 110

9.4 Sensitivity of the sensors to the sound particle velocity . . . . . . . . . 111 
10 Influence of 3D effects on a nonlinear AWC.

113

11 Extension to the demands on an AWC system

119

11.1 Observability . . . . . . . . . . . . . . . . . . 119

11.1.1 Acoustic sensitivity of the sensors . . . . . . . . . . . . . . 119

11.1.2 Wall shear stress dependence of the modes . . . . . . . . . . . 120

11.2 Controllability . . . . . . . . . . . . . . . . 120

12 Summary, conclusions, and outlook 123

$\begin{array}{ll}\text { Bibliography } & 131\end{array}$

$\begin{array}{ll}\text { Acknowledgement } & 136\end{array}$ 


\section{List of Figures}

2.1 The dispersion characteristics of 3D TS waves in Blasius flow obtained by spatial stability analysis using spectral methods (carried out by Boiko $(2000)) . \ldots \ldots \ldots \ldots \ldots$

2.2 Eigenfunctions of the streamwise velocity component $|u|$ (normalized to one) for three different spanwise wavenumbers at $\mathrm{F}=120, \mathrm{Re}=420$ (marked in Fig. $2.1(\mathrm{a})$ ). . . . . . . . . . . . . . . . . . .

2.3 Dispersion characteristics of 3D TS waves for the Blasius flow. A comparison between experimental and theoretical investigations (from Kachanov (1995)) . . . . . . . . . . . . . . . . . . 10

3.1 The schematic sketch of the cancellation device. . . . . . . . . . . . . . 14

3.2 Control strategy in terms of continuous temporal-spatial spectra. In the following, the quantities at the streamwise location of the reference plane are denoted by an index $r$, similarly for the error plane by an index $e$. .

3.3 A general diagram of a sensor-actuator-sensor configuration and its notation, here with equal spanwise spacing for its components. The configuration with $J=K=M$ will be referred to as symmetric configuration. 18

3.4 Signal flow diagram of the system in terms of transfer matrices . . . . . 19

3.5 Refinement of the signal flow diagram of the system in terms of transfer matrices. . . . . . . . . . . . . . . . . . 23

4.1 The principal scheme of an adaptive filter. . . . . . . . . . . . . . . 31

4.2 The system identification scheme for estimation of the secondary path. 35

4.3 The active wave cancellation scheme for a single reference sensor, actuator, and error sensor. . . . . . . . . . . . . . . 36

4.4 The block diagram of the filtered-X-LMS algorithm for active wave cancellation. . . . . . . . . . . . . . . . . . . 
4.5 Scheme of the MIMO active wave cancellation system consisting of 2 reference sensors, 2 actuators (sharing one slit), and 2 error sensors. The 4 adaptive filters (marked in green) are optimized by the 2-input/2output filtered-X-LMS algorithm. . . . . . . . . . . . . .

5.1 The low-turbulence wind tunnel TUG of the DLR Göttingen (side view and top view, all dimensions in meters). . . . . . . . . . . . 44 46

5.2 Side view and top view of the plate, all dimensions are given in millimeters. 47

5.3 Spectrum (PSD) of a typical hot-film sensor signal showing the reduction of electrically induced noise by adaptive filtering. . . . . . . . . . . .

6.1 Actuator Type I: Schematic spanwise pressure distribution and simplified top view (left). A cross section of the actuator in streamwise direction, all dimensions in millimeters (right). . . . . . . . . . . .

6.2 Comparison of SISO AWC system applied to 'natural' (left side) and 'artificial' (right side) TS-waves. . . . . . . . . . . . . . . . . . .

6.3 Lateral correlations of 'natural' TS-waves with and without the control by the SISO AWC system.

6.4 The spanwise RMS-distribution (100-260 Hz) of two spanwise independent SISO-AWC systems applied to 'artificial' 2D TS-waves (measured at $x=74 \mathrm{~cm}) \ldots \ldots \ldots \ldots \ldots \ldots \ldots \ldots$

6.5 The influence of the roughness caused by the disturbance generator (DG) slot on the estimation of $\mathbf{S}, \Delta x_{a e}=6 \mathrm{~cm} \ldots \ldots \ldots$

6.6 Spectra and coherence measurements indicating the receptivity of the disturbance generator slot. . . . . . . . . . . . . . . . 61

6.7 Wall-hot wire sensor as described in Baumann (1999). . . . . . . . . . 63

6.8 Side view and top view of the plate including the cancellation device (Configuration Type II), all dimensions are given in millimeters. . . . . 64

6.9 Actuator Type II: Schematic spanwise pressure distribution and simplified top view (left). A cross section of the actuator in streamwise direction, all dimensions in millimeters (right). . . . . . . . . . . .

6.10 Spatial frequency spectra for the different actuators. The spanwise wavenumber is divided by the Nyquist wavenumber of the sensor configuration and the colors indicate the logarithmic scale, given in decibels. 66

6.11 Streamwise development of the actuator frequency and wavenumber spectrum, $U_{\infty}=18 \mathrm{~m} / \mathrm{s} \ldots \ldots \ldots \ldots \ldots$ 
7.1 Variation of the pressure gradient for $U_{\infty}=18 \mathrm{~m} / \mathrm{s} \ldots \ldots \ldots$

7.2 Comparison of the experimental data to the Blasius solution. . . . . . . 71

7.3 The stability diagram for 2D disturbances in Blasius flow, the device components are marked for $U_{\infty}=18 \mathrm{~m} / \mathrm{s} \ldots \ldots \ldots$. . . . . . .

7.4 Streamwise development of the mean velocity profiles and the RMSprofiles for natural TS-waves and for the excitation by the point source (PS). The profiles are measured at the centerline $(z=0)$ and the wall normal coordinate is normalized by $\delta_{1}$. Note that the scales of $u^{\prime} / U_{\infty}$ differ between the diagrams for the different x-locations. . . . . 77

7.5 Set-up and system identification scheme in the point-source experiment. 78

7.6 Downstream development of a normalized pulse disturbance described by the assembled impulse responses $W_{1}(z) \ldots \ldots \ldots \ldots$

7.7 Spanwise magnitude (left) and phase (right) distribution of the transfer function between the indicated streamwise locations. . . . . . . . . .

7.8 Phase and group velocity versus frequency. The error bars denote the standard deviation of the average calculated for the region $20 \mathrm{~cm} \leq x \leq$ $50 \mathrm{~cm}$. . . . . . . . . . . . . . . . 82

7.9 Spatial stability characteristics of the investigated boundary layer. . . . 82

7.10 Downstream development of a point-source disturbance pulse $\left(W_{1}(z)\right)$ in the frequency-wavenumber domain (for details see table 7.1). . . . .

7.11 RMS-spectra of the point-source disturbance for different excitation amplitudes, at $x=72 \mathrm{~cm} \ldots \ldots \ldots \ldots \ldots \ldots$

7.12 Qualitative comparison between the correlated streamwise velocity fluctuations of the measured pulse and a pulse obtained by a DNS from Konzelmann (1990) (negative values are marked by the dashed isolines).

8.1 Adapted filters modelling the transfer matrix $\mathbf{S}$ between the actuators and the error sensors (left) and the transfer matrix between the reference sensors and the actuators (right) at $U_{\infty}=18 \mathrm{~m} / \mathrm{s} \ldots \ldots . . . . .$.

8.2 Application of the AWC system to naturally occurring TS-waves operating in two different control modi. . . . . . . . . . . . . . . . .

8.3 Spectra and coherence showing the performance of the AWC applied to natural TS-waves. . . . . . . . . . . . . . . . . . 93

8.4 Spanwise coherence of naturally occurring disturbances. . . . . . . . . . 95 
8.5 Spatial impulse response between the acoustic source in the settling chamber of the wind tunnel and a spanwise traversed hot-wire probe, illustrating the acoustic receptivity of the point-source slot $(z=0 \mathrm{~cm})$. The colors indicate the pulse amplitude. . . . . . . . . . . . . . . . 96

8.6 Comparison between the excitation by the point source and the one by a loudspeaker. . . . . . . . . . . . . . . .

8.7 Left side: Spatial pulse of a point source disturbance measured at $x=74 \mathrm{~cm}$ for $U_{\infty}=18 \mathrm{~m} / \mathrm{s}$ uncontrolled (a), controlled by independent channels (c), controlled by coupled channels (e), and the related spanwise wavenumber spectra (right side). . . . . . . . . . . . . . . 101

9.1 The influence of sound and vibrations on the modelling by a SISO-AWC system. . . . . . . . . . . . . . . . . . 105

9.2 The influence of the roughness of the actuator slot on $H_{r e} \quad \ldots \ldots 8$

9.3 Spectra and correlations for a SISO AWC system in a noisy environment.109

9.4 Schematic diagram of the acoustic feedback effect. . . . . . . . . . . . . 111

10.1 AWC of artificial broadband disturbances taking into account the occurring nonlinear effects. . . . . . . . . . . . . . . . . 114

10.2 Nonlinear wave cancellation using an error signal detected at the wall. (from Evert (2000)) . . . . . . . . . . . . . . 115

10.3 Nonlinear wave cancellation using an error signal detected in the boundary layer. (from Evert (2000)) . . . . . . . . . . . . . 115

10.4 Linear stability characteristics of the investigated boundary layer concerning 3D disturbances. (obtained by a linear stability solver from A.V.Boiko described in (Boiko (2000)) . . . . . . . . . . . 117 


\section{List of Tables}

7.1 Boundary layer parameters: $\delta_{1}, \delta_{2}, H_{12}, U_{e}, R e_{\delta 1}$ and $R e \ldots . . . .72$

8.1 The standard deviation for different values of the coherence function $\gamma_{r e}^{2}$ evaluated for $n_{a v}=300 \ldots \ldots \ldots \ldots . \ldots \ldots$

9.1 Schematic overview of several propagation processes identified in the correlation data. The experimentally determined $\left(\left\{\int c_{g r} \mathrm{~d} t\right\}_{\text {exp }}\right)$ and the theoretically expected $\left(\left\{\int c_{g r} \mathrm{~d} t\right\}_{\text {theo }}\right)$ travelling distances are compared for the different propagation processes. Abbreviations: DG: disturbance generator, RS: reference sensor, A: actuator, ES: error sensor. . . . . 107 


\section{List of symbols}

\section{Latin Letters}

$\begin{array}{ll}A(\beta, \omega) & \text { transfer function between the reference plane and the error plane } \\ \mathbf{A} & \text { transfer matrix between the reference sensor and the error array } \\ A, B, n & \text { calibration constants in King's Law } \\ F & \text { partial derivative operator } \frac{\partial}{\partial y} \\ F & \text { the non-dimensional frequency, } F=\omega \nu / U_{\infty}^{2} \\ J, K, M & \text { number of reference sensors, actuators and error sensors } \\ N_{\max } & \text { maximum number of degrees of freedom of the disturbance wave-field } \\ \mathbf{p} & \text { cross-correlation vector (matrix) between the reference sensor signal(s) } \\ U_{e} & \text { and the error sensor signal(s), SISO (MIMO)-system } \\ U_{\infty} & \text { velocity at the boundary layer edge } \\ W(\beta, \omega) & \text { freestream velocity } \\ \mathbf{W} & \text { transfer function between the reference plane and the control plane } \\ \mathbf{R} & \text { transfer matrix between the reference sensor and the actuator array } \\ R e_{x} & \text { autocorrelation matrix of the reference sensor signal(s), SISO (MIMO)-sys. } \\ R e & \text { Reynolds number based on the distance } \\ R e_{\delta_{1}} & \text { from the leading edge } R e_{x}=\frac{U_{\infty} x}{\nu} \\ S(\beta, \omega) & \text { Reynolds number based on Blasius length, } R e=\sqrt{R_{x}}=U_{\infty} \delta / \nu \\ \mathbf{S} & \text { Reynolds number based on the local displacement thickness } \\ T(x, \beta, \omega) & \text { transfer function between the control plane and the error plane } \\ & \text { transfer matrix between the actuator array and the error sensor array } \\ & \text { frequency-wavenumber spectrum at streamwise location } x, \\ c_{e} & T(x, \beta, \omega)=\mathcal{F}_{t, z}\{\tau(x, z, t)\} \\ c_{g r} & \text { envelope velocity } \\ c_{p h} & \text { group velocity, } c_{g r}=\text { d } \omega / \mathrm{d} k \\ f & \text { frequency } \\ f_{s} & \text { sampling frequency }\end{array}$


$t \quad$ time

$x, y, z \quad$ streamwise, spanwise, and wall-normal coordinate

\section{Greek Letters}

$\begin{array}{ll}\alpha & \text { streamwise wavenumber, } \alpha \in \mathbb{C}, \alpha=\alpha_{r}+i \alpha_{i} \\ \beta & \text { spanwise wavenumber, } \beta \in \mathbb{R} \\ \beta^{*} & \text { dimensional spanwise wavenumber } \beta \\ \Delta \beta_{\max } & \text { maximum } \beta \text { contained in the disturbance spectrum } \\ \Delta z_{\max } & \text { maximum spanwise correlation length of the disturbance wave-field } \\ \delta & \text { Blasius length, } \delta=\sqrt{\frac{\nu x}{U_{\infty}}} \\ \delta_{99 \%} & \text { boundary layer thickness, } 99 \% \\ \delta_{1} & \text { local displacement thickness } \delta_{1}=\int_{0}^{\infty}\left(1-U(y) / U_{\infty}\right) d y \\ \delta_{2} & \text { local momentum thickness } \delta_{2}=\int_{0}^{\infty} U(y) / U_{\infty}\left(1-U(y) / U_{\infty}\right) d y \\ \nu & \text { kinematic viscosity } \\ \mu & \text { step-size parameter in the LMS algorithm } \\ \rho & \text { density of air } \\ \omega & \text { circular frequency, } \omega=2 \pi f \\ \tau_{W} & \text { wall shear stress } \\ \tau(x, z, t) & \text { temporal-spatial disturbance distribution at streamwise location } x, \\ & \tau(x, z, t)=\mathcal{F}_{\omega, \beta}^{-1}\{T(x, \beta, \omega)\}\end{array}$

\section{Formular Symbols}

$\begin{array}{ll}\mathbf{X} & \text { matrix } \mathbf{X} \\ \mathbf{x} & \text { vector } \mathbf{x} \\ \Re\{X\} & \text { real part of } X \\ \Im\{X\} & \text { imaginary part of } X \\ <X> & \text { expectation operator } \\ |X| & \text { modulus of } X \\ \bar{X} & \text { time average of } X \\ \hat{X} & \text { estimator of } X \\ X^{*} & \text { conjugate complex of } X \\ X^{T} & \text { the transpose of } X \\ X^{H} & \text { hermitian of } X, \text { the transpose of the conjugate complex } \\ \mathcal{F}_{v}\{X\} & \text { Fourier transform of } X \text { with respect to } v \in\{t, z, f, \beta\} \\ \nabla & \text { Nabla operator }\end{array}$




\section{Indices}

$\begin{array}{ll}a & \text { actuator } \\ e & \text { error sensor } \\ N y & \text { Nyquist (wavenumber) } \\ r & \text { reference sensor } \\ \text { rms } & \text { root-mean-square value }\end{array}$

\section{Abbreviations}

AWC

ENR

LMS

LTI

MIMO

PSD

SISO

sFTF

SNR

TS

RMS active wave cancellation

electrical induced noise reduction

least mean square

linear time invariant

multiple-input-multiple-output

power spectral density

single-input-single-output

stabilized fast transversal filter

signal-to-noise ratio

Tollmien-Schlichting

root-mean-square 


\section{Chapter 1}

\section{Introduction}

\subsection{The relevance of the subject}

The delay of the laminar to turbulent transition is crucial for the reduction of the viscous part of the drag as experienced by bodies in a flow. The increased drag results from the high mixing in turbulent boundary layers which causes an increased momentum loss and energy dissipation.

Among others aircraft industries have a great interest to reduce the enormous fuel consumption of modern aircrafts (e.g. the one of an Airbus A320 of about 5 liters of Kerosin per 100 kilometres per person). The potential savings can be estimated by the fact that up to $50 \%$ of the total drag are caused by viscous drag and by the fact that the drag caused by a laminar boundary layer is only the tenth part of the turbulent one at the same Reynolds number (Bushnell 1990; Joslin 1998).

Among these technical reasons, the scientific interest in the phenomenon of turbulence itself led to a high interest of many engineers and scientists who have been working on the laminar-turbulent transition within the last decades. The origin of turbulence at the swept transonic wing, which is a standard for transonic speeds in civil aviation, arises from different mechanisms. It starts with the leading-edge instability followed by the cross-flow instability that is caused by an inflection point of the spanwise velocity profile. Without precautions these instabilities lead to turbulence within a few centimeters from the leading edge (depending on the sweep angle of the wing). It has been shown theoretically and experimentally that these instabilities can be suppressed by boundary layer suction. Lately, this has even been applied successfully to the fin of an Airbus A320 (Henke 1999).

Numerous experimental and theoretical studies are centered around this method which is known as active flow control. With respect to the fact that stabilization 
is achieved here by influencing the mean velocity-profile directly due to the applied suction (similar to the design of the wing profiles), this method could more suitably be called a passive method in the sense of the present work. In other words there is no active reaction to the actual state of the boundary layer.

Provided a successful delay of the laminar-turbulent transition in the front part of the wing is accomplished, the Tollmien-Schlichting instability becomes the dominating cause of transition in the rear part enhanced by an adverse pressure gradient. Disturbances of the mean-profile excited by external perturbations propagate downstream while getting amplified or damped depending on the frequency, the Reynolds number, and the spanwise wavenumber. First, Tollmien (1929) predicted the existence of these convective instability waves by an analytic stability analysis. Schlichting (1933) published the first calculus of their growth rates. More than one decade later Schubauer and Skramstad (1948) proved the existence of these Tollmien-Schlichting waves experimentally. On the way downstream the amplitude of the Tollmien-Schlichting waves is further increased and a secondary instability comes into play (Klebanoff et al. 1962), which causes a three-dimensional distortion of the mean flow leading to the generation of lambda-structures. A possible way of the transition to a fully turbulent flow is then initiated by the occurrence of streaky structures and turbulent spots.

One possibility to delay transition is to use the stabilizing effect of a negative streamwise pressure gradient, which leads to the development of the laminar wing profiles whereby the pressure minimum is shifted towards the rear of the wing. The combination of this technique with boundary layer suction at the leading edge is called Hybrid-Laminar-Flow-Control. In contrast to these methods that are based on the modification of the mean flow profile, the method investigated here aims at the delay of transition by a direct adaptive interaction with the disturbance waves initiating the process. This active wave cancellation (AWC) method is based on the wave superposition principle, i.e. to superimpose artificially generated counter-phase disturbances on the naturally occurring disturbances in order to cancel these.

\subsection{Previous experimental studies in the field}

First experiments were carried out by Wehrmann (1965) and Schilz (1965) and demonstrated that a single frequency TS-wave produced by a disturbance generator (vibrating ribbon and oscillating piston, respectively) could be cancelled by an active flexible wall. The generated propagating flexural wave was adjusted to the TS-wave in the boundary layer, namely in wavelength, amplitude, and phase, and a decrease of the velocity fluctuations have been observed for appropriate settings at a sensor further downstream. In both investigations a noticeable delay of transition was observed. 
Milling (1981) and Liepmann and Nosenchuk (1982) carried out a similar experiment in water and similarly observed that a single frequency TS-wave produced by a disturbance generator could be cancelled by a second TS-wave generated at an actuator further downstream. The magnitude and phase of the compensating TS-wave were adjusted manually to minimize an error signal detected further downstream. Both experiments have been carried out with a flat plate in a water channel and the TS-waves were generated by a vibrating ribbon and a periodically heated surface element, respectively. With dye visualization technique, Milling observed a shift of the transition location from $R e_{x}=5.2 \times 10^{5}$ beyond the trailing edge of the plate $\left(R e_{x}>6.7 \times 10^{5}\right)$, caused by the cancellation of the primary disturbance. In realistic transition scenarios the primary disturbances are unknown and therefore have to be detected upstream of of the actuator by an additional sensor, known as reference sensor. In order to calculate the optimum compensation signal, it is necessary to model accurately the propagation between the reference and error sensor of these naturally occurring disturbances that are generally of broadband nature. Despite of this fact, the early publications in the field reduced the physical model of propagation to a simple delay and an amplification independent of frequency, which restricted the achieved cancellation mainly to single frequencies (Thomas 1983; Liepmann and Nosenchuck 1982).

The cancellation of broadband disturbances was first demonstrated by Pupator and Saric (1989), who eliminated the distorting influence of the electro-mechanical devices by filtering the reference signal with the inverse transfer function of the electromechanical path. This improved the model significantly (Thomas 1990). Ladd and Hendricks E.W. (1988) presented a completely new approach modelling the propagation by means of a linear adaptive digital filter. For non-obvious reasons this method was only applied to an artificially generated single frequency wave, whereas later cancellation experiments of naturally occurring TS-waves were carried out with a simple model of a delay and an amplification adjusted by a simple optimization algorithm (Ladd 1990). A good overview of the work referenced so far is given by (Thomas 1990).

Some newer experimental work in the field of active wave cancellation has been performed by Raguse (1998), and Evert (2000) at the German Aerospace Center DLR in Göttingen as well as by Baumann (1999) and Sturzebecher and Nitsche (2002) at the TU Berlin. The investigations of Raguse and Baumann concentrated on the application of linear adaptive filters to the modelling problem in the boundary layer of a flat plate and a wing-like profile, respectively. In both investigations remaining disturbances have been observed in the frequency range of the Tollmien-Schlichting instability that could not be eliminated by the active wave cancellation. For this reason Evert (2000) focussed on the nonlinear modelling of the disturbance propagation process in a flat plate boundary layer. He developed a nonlinear active wave cancellation system that was applied to two-dimensional broadband TS-waves of high amplitude that have been generated by means of acoustic blowing and suction. The disturbances remaining after 
linear control have been affected by the nonlinear method. However, it turned out that the wall normal profile of the nonlinearly controlled disturbances was different from the known profile of two-dimensional Tollmien-Schlichting waves.

In direct numerical simulations (DNS), Gmelin (2001) was able to damp disturbances in the nonlinear stage of transition by instantaneous feedback of the $\pi / 2$-phaseshifted spanwise vorticity component to the wall-normal velocity that was generated by a disturbance strip. Herein, a continuous distribution of both quantities was evaluated over a streamwise distance of about 5 times the TS-wavelength. The mechanism of this approach is somewhat different from the wave cancellation methodes in so far as the boundary layer is stabilized by changing boundary conditions at the wall. This active wall has not yet been realized experimentally for broadband TS-waves.

According to Squire's theorem (Squire 1933), namely that two-dimensional waves will be amplified first, the above mentioned investigations concentrated on the control of this two-dimensional component. Even though these two-dimensional waves are dominating the linear stage of transition, the initially weaker three-dimensional components may be most important in the nonlinear breakdown. No general statement can be given on the influence of the three-dimensional disturbances on the breakdown to turbulence. Direct numerical simulation (DNS) modelling of different breakdown scenarios in a boundary layer of a wing by Stemmer (2001) showed that the streamwise position of the breakdown did not shift due to filtering out the two-dimensional wave in the incident spectra. Furthermore he concluded that two-dimensional disturbances do not play any leading role, rather they are a side effect.

\subsection{The objectives of this investigation}

The objective of this study is to investigate the active wave cancellation method with respect to simultaneously occurring two-dimensional (2D) and threedimensional (3D) waves. Of special interest is the question, if it is necessary to treat $2 \mathrm{D}$ and $3 \mathrm{D}$ waves differently. In order to design an active wave cancellation device that can adequately cope with the differences of these disturbances, the demands to such a device must be specified. The angle dependence of the dispersion relation will influence such demands and needs therefore to be investigated as well.

Simultaneously to the present work, Sturzebecher approached the control of 3D waves in a wing-like boundary layer by lining-up a number of 2D-systems in spanwise direction with small distances in between. In contrast to the present study, he did not distinguish between 2D and 3D waves. His results will be cited at appropriate places in the text. 


\section{Chapter 2}

\section{Dynamics of the boundary layer}

In fluids, turbulent rather than laminar motion is observed mostly because the Reynolds-number range of laminar motion is generally limited. The process of laminarto-turbulent transition starts far upstream of the breakdown to turbulence. The onset of the transition scenario is outlined in this chapter.

\section{$2.1 \quad$ Receptivity}

Disturbances in the free stream, such as sound or vorticity, enter the boundary layer as steady and/or unsteady disturbances of the basic state. Generally, an unsteady external perturbation is scattered at non-uniformities in the boundary layer, thereby generating an unsteadiness in the boundary layer with a broad wavenumber spectrum and a part of these will match such wavenumbers found to be unstable in the considered boundary layer.

A variety of different excitation processes are known, e.g. the scattering of longwavelength sound waves at roughness elements or other non-uniformities of the wall surface, or short-wavelength pressure fluctuations due to free-stream turbulence. Also surface vibrations in connection with surface non-uniformities and the interaction of sound or vibrations with steady free-stream non-uniformities are a possible source of these fluctuations as well as nonlinear interactions between all these sources.

The mechanisms by which forced disturbances enter the laminar boundary layer are usually addressed by the word receptivity, named by Morkovin (1968). Although these mechanism are still not well understood, they provide the vital initial conditions of amplitude, frequency and phase for the breakdown of the laminar flow. Initially these disturbances may be too small to measure and they are observed only after the onset of the instability. 
Over the decades these processes were investigated by several scientists. Only extracts of a few investigations which are of interest for the present work are mentioned here.

Gilyov and Kozlov (1984) studied experimentally the generation of TS-waves by 2D surface vibrations and found that a disturbance source placed directly at the wall excites 2D TS-waves rather effectively.

A comparison of the theoretical and experimental results concerning receptivity was performed by Ivanov et al. (1998). Very good qualitative agreement was found, namely, the growth of receptivity with frequency of surface vibrations, and a higher receptivity of the Blasius boundary layer to 3D perturbations versus 2D waves.

In further theoretical and experimental investigations (Kerschen et al. 1990) it was shown that a boundary layer is strongly receptive in regions where it is subject to streamwise velocity gradients, i.e. at the leading edge or at local surface nonuniformities (e.g. hump, step or roughness).

Neemann et al. (2001) presented experimental and theoretical investigations concerning the receptivity of boundary layers to $3 \mathrm{D}$ wall vibrations. They found that the receptivity of the Blasius boundary layer to $3 \mathrm{D}$ wall vibrations is higher than to $2 \mathrm{D}$ vibrations. This angle dependence becomes weaker for adverse pressure gradients. Furthermore the vibrational receptivity mechanism is compared to the receptivity due to blowing or suction at the wall, which led to the conclusion that the latter is 1-2 orders of magnitude higher than the vibrational one.

Ivanov et al. (2001) used a modulation technique to extract the 3D acoustic receptivity in the presence of microscopic surface vibrations. They found that external acoustic perturbations are transformed more effectively into $3 \mathrm{D}$ waves than into $2 \mathrm{D}$-waves and that this difference in receptivity becomes more significant for higher frequencies of the TS-waves.

From recent results in receptivity research one may conclude: The more stable the boundary layer is with respect to a specific disturbance the more receptive it is also.

\subsection{Linear instability}

Once the perturbations have found their way into the boundary layer being transformed to small amplitude waves, they are either linearly damped or amplified while propagating downstream. This process can be described theoretically by classic, linear stability analysis which leads to the well known Orr-Sommerfeld equation, given here for threedimensional waves in a two-dimensional flow (for the derivation see e.g. Cousteix (1992) 


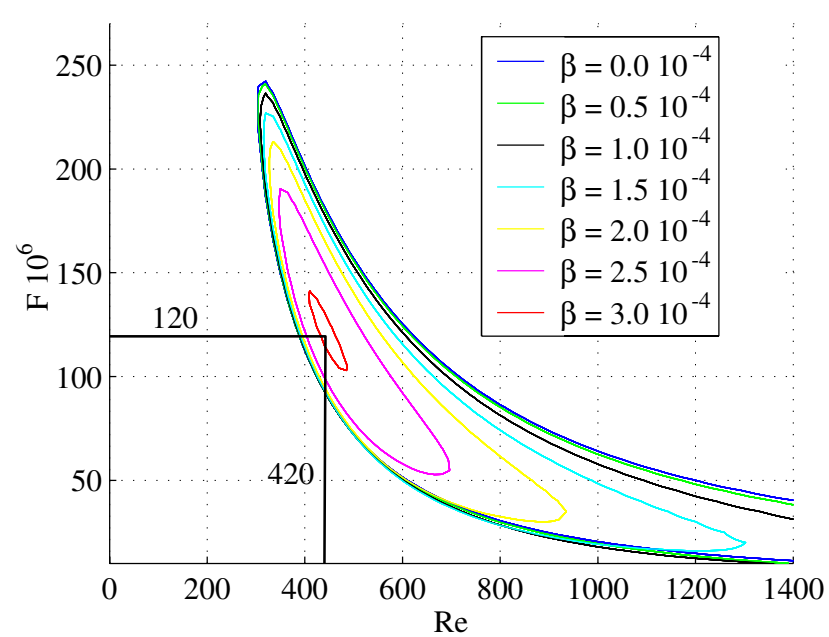

(a) Neutral stability curves are plotted for different spanwise wave numbers $\beta=\beta^{*} \nu / U_{\infty}$ in the plane spanned by the non-dimensional frequency $F$ and the Reynolds number $R e$.

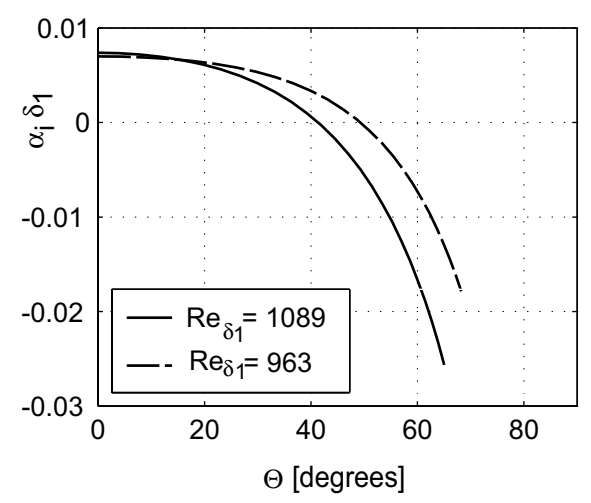

(b) The growth rates $\alpha_{i} \delta_{1}$ are plotted versus the propagation angle for two Reynolds numbers at the nondimensional frequency $F=94.1 \times 10^{-6}$. Compare with Fig. 2.3 (c).

Figure 2.1: The dispersion characteristics of 3D TS waves in Blasius flow obtained by spatial stability analysis using spectral methods (carried out by Boiko (2000)).

or Mack (1984)):

$$
\left(D^{2}-\alpha^{2}-\beta^{2}\right)^{2} \tilde{v}=i \operatorname{Re}\left[-\left(\alpha D^{2} U\right)+(\alpha U-\omega)\left(D^{2}-\alpha^{2}-\beta^{2}\right)\right] \tilde{v},
$$

where $D$ stands for the operator $\frac{\partial}{\partial y}$ applied to the wall normal velocity component $\tilde{v}$ and $R e$ denotes the Reynolds number with respect to the Blasius length $\sqrt{\frac{\nu x}{U_{\infty}}}$.

Due to the linearity of equation (2.1) and its the homogeneity in the variables $x, z$, and $t$ a wave-Ansatz can be made such that a solution for $\tilde{v}$ can be computed. Thus, the streamwise velocity component $\tilde{u}$ can be obtained using a procedure e.g. described in Boiko, Grek, Dovgal, and Kozlov (2002). Hence, the streamwise velocity component $\tilde{u}$ is of the form

$$
\tilde{u}=\tilde{u}_{0}(y) e^{i(\alpha(\omega, \beta, R e) x+\beta z-\omega t)}, \quad \beta, \omega \in \mathbb{R}, \alpha \in \mathbb{C}
$$

wherein the dispersion relation $\alpha(\omega, \beta, R e)$ describes the dynamics of the boundary layer. The well known stability diagram, i.e. the curves of zero-amplification $(\Im\{\alpha\}=0)$ in the plane spanned by the Reynolds number $R e$ and the non-dimensional 


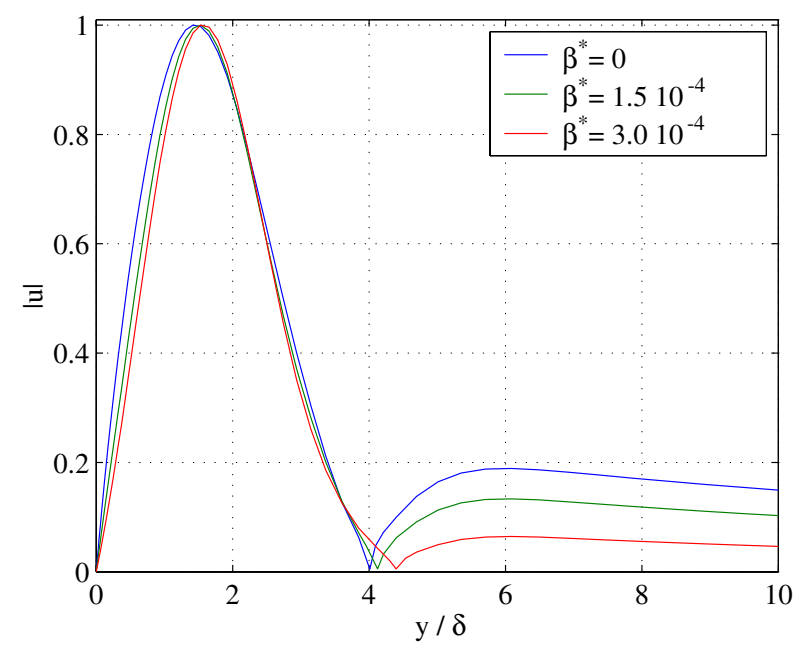

Figure 2.2: Eigenfunctions of the streamwise velocity component $|u|$ (normalized to one) for three different spanwise wavenumbers at $\mathrm{F}=120, \mathrm{Re}=420$ (marked in Fig. 2.1(a)).

frequency $F=\omega \nu / U_{\infty}^{2}$, is shown in Fig. 2.1 a) for different values of the spanwise wavenumber $\beta$ (also in non-dimensional form $\beta=\beta^{*} \nu / U_{\infty}$, where $\beta^{*}$ is the dimensional spanwise wavenumber). The critical Reynolds number increases with higher values of the spanwise wavenumber $\beta$ (according to the Squire theorem) and the unstable region becomes smaller. Fig. 2.1 (b) shows the dependence of the growth rate on the propagation angle $\theta$ and indicates that oblique waves up to $\theta \approx 40^{\circ}$ are growing. The comparison to Fig. 2.3(c) reveals that the results agree well with the ones obtained by other authors.

In Fig. 2.2 the eigenfunctions of the streamwise velocity component $|u|$ are plotted for the tuple $(\mathrm{F}=120, \mathrm{Re}=420)$ indicated by the marked point in the stability diagram of Fig. 2.1 (a). The figure reveals that for increasing the spanwise wavenumber, the inner maximum shifts slightly away from the wall and the outer maximum significantly decreases. Related to the outward shift of the inner maximum is a decrease of the gradient at the wall and thereby of the wall-shear stress fluctuations $\dot{\tau}_{W}=\left.\mu \frac{\partial u}{\partial y}\right|_{y=0}$.

Pressure gradients influence the stability characteristics of the boundary layer, significantly. The effect of pressure gradients on the stability diagram can be obtained by solving the Orr-Sommerfeld equation for the Falkner-Skan velocity profiles characterized by the shape parameter $H$. The shape parameter for Blasius condition is $H=2.59$. The neutral curve is wider for positive pressure gradients $(H>2.59)$ and when the Reynolds number tends to infinity the range of the unstable frequencies is non-zero: a positive pressure gradient is destabilizing. The reason for this destabiliza- 
tion lies in an inflection point of the velocity profile, what was shown first by Rayleigh. He evaluated the equation, named after him,

$$
\left((\alpha U-\omega)\left(D^{2}-\alpha^{2}\right)-\left(\alpha D^{2} U\right)\right) \tilde{v}=0
$$

which can be obtained by neglecting the viscosity in the Orr-Sommerfeld equation for $\beta=0$. Thus, this inflectional instability is called non-viscous instability and plays an important role in stability theory. The critical Reynolds number decreases very much with a positive pressure gradient: for the separation velocity profile $(H=4.029)$ $R e_{\delta 1}=67$, whereas for $H=2.216 R e_{\delta 1}=12490$ is found (Cousteix 1992).

\subsection{Dispersion characteristics of oblique waves}

Kachanov (1995) gives an overview of the experimental and theoretical investigations that have been carried out within the last decades concerning the 3D instability waves in boundary layers. This section mainly summarizes the results which are of importance for the present work. The first successful experimental investigation of 3D instability waves in the flat-plate boundary layer was conducted by Gaster and Grant (1975) and the results of this study were compared with calculations by Gaster (1975) and later with a direct numerical simulation (DNS) by Konzelmann (1990).

A complete set of stability characteristics of the Blasius boundary layer was obtained 1983 by Gilyov ${ }^{1}$, and later additionally processed for comparison with parallel linear stability theory (PLST) by Kachanov and Michalke (1994). Another set of experiments in Blasius flow was conducted by Kachanov and Obolentseva (1996) in order to investigate in more detail the stability characteristics, non-parallel effects, as well as the angle-dependence of the eigenfunctions of 3D TS modes. In both experiments disturbances, harmonic in time, were introduced into the boundary layer by means of localized disturbance sources of two types, namely by a point source and by a circular membrane-type vibrator. In both cases, in the data processing the wave trains were decomposed into normal oblique TS waves inclined at various angles with respect to the flow direction, as described in detail by Kachanov and Michalke (1994).

The dependence of the non-dimensional streamwise wavenumber $\alpha_{r} \delta_{1}$ on the wave angle $\theta=\tan ^{-1}\left[\beta / \alpha_{r}(\beta)\right]$ is shown in Fig. 2.3 (a) for three different frequency parameters $F=\omega \nu / U_{o}^{2}$. The quantitative agreement between the experimental and theoretical results is good for a wide range of wave angles, but some deviations are found at very large angles $\left(\theta>50^{\circ}\right)$. Also a good agreement has been obtained for the downstream phase velocity $\left(C_{x}=\omega / \alpha_{r}\right)$ shown in Fig. $2.3(\mathrm{~b})$

\footnotetext{
${ }^{1}$ See (Kachanov 1995) and references therein.
} 


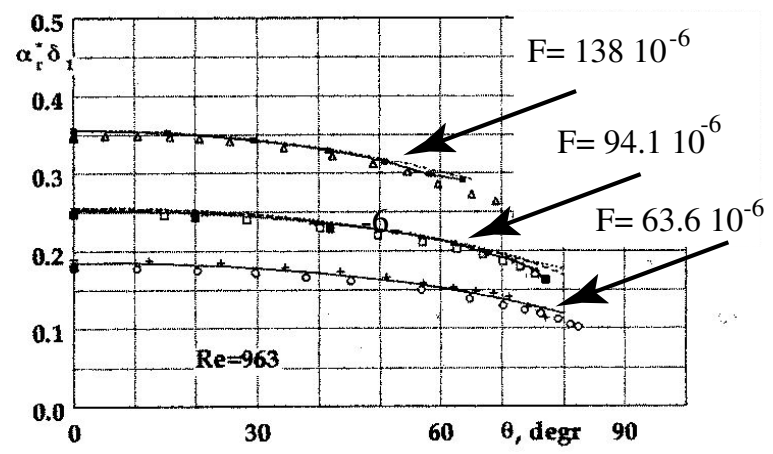

(a) Streamwise wavenumbers versus wave angle (see Kachanov (1995) for details).

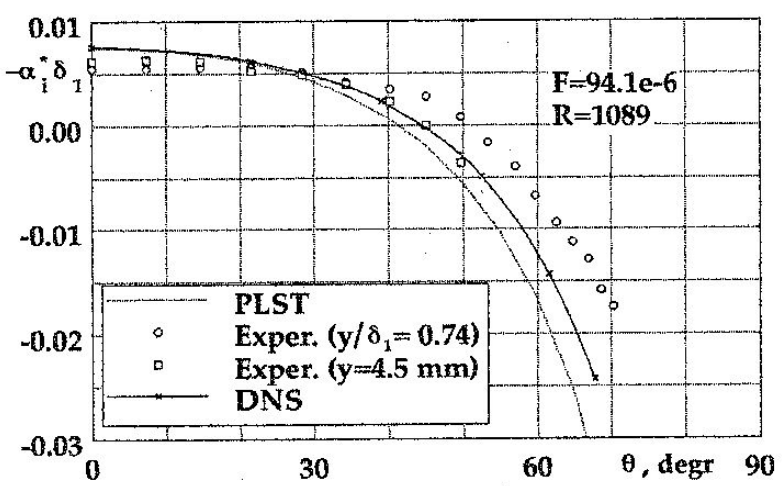

(c) Growth rate versus propagation angle.

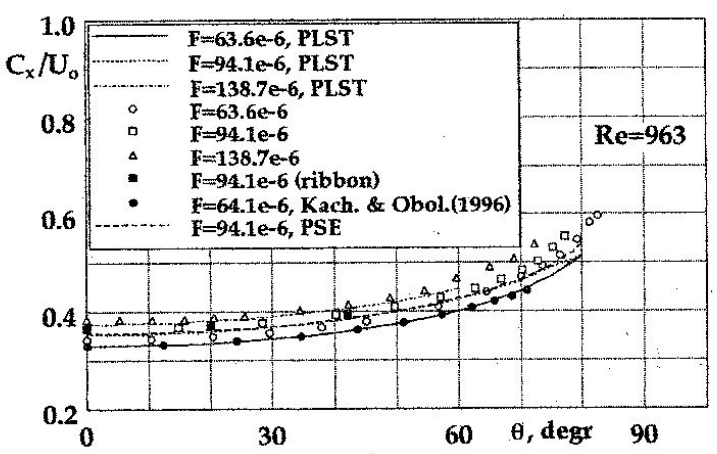

(b) Phase velocities versus propagation angle.

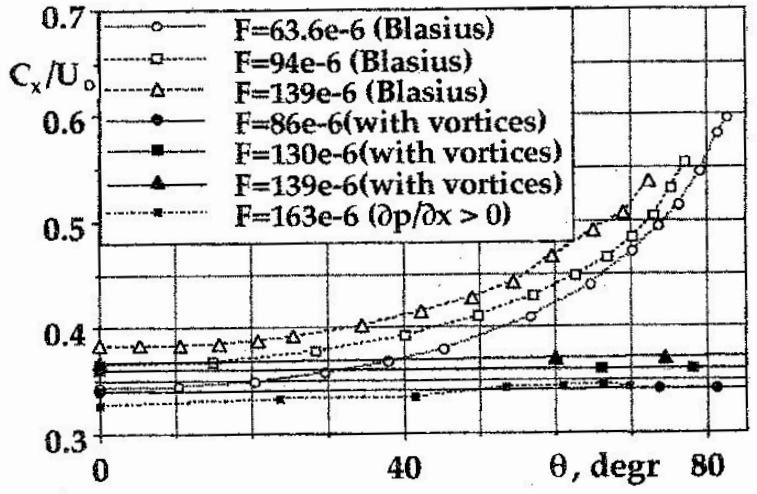

(d) The influence of streamwise vortices and pressure gradients on the phase velocity.

Figure 2.3: Dispersion characteristics of 3D TS waves for the Blasius flow. A comparison between experimental and theoretical investigations (from Kachanov (1995))

The theoretical prediction of the growth rates $\left(-\alpha_{i}\right)$ represents a much more complicated problem. Kachanov (1995) states, Good quantitative agreement for $-\alpha_{i}$ between experimental and theoretical values has never been obtained for the flat-plate boundary layer; not for 3D modes, and not even for $2 D$ modes. A comparison of experimentally and theoretically (PLST by Michalke and DNS by Ebert) obtained growth rates is shown in Fig. 2.3 (c). There are several causes for the discrepancies between experimental and theoretical growth rates. One of them is the dependence of the shape of 
the eigenfunctions on the wave angle, (Bertolotti 1991).

Gilyov et al. investigated the dispersion characteristics of 3D TS waves in the boundary layer of an airfoil. In contrast to the Blasius flow they found the streamwise wavenumber to be independent of the spanwise wavenumber (not considering the growth rate) in the region of adverse pressure gradient $(\partial p / \partial x>0)$, while in the region of favorable pressure gradient $(\partial p / \partial x<0)$ this dependence becomes even stronger than in the Blasius flow, and in contrast to the first case it depends significantly on the Reynolds number and the local pressure gradient.

Kachanov (1995) concluded from the observations in the experiments that the disappearance of the dispersion of 3D modes is associated with the prevailing role of the inviscid instability of the flow with inflectional profiles, i.e. that the most unstable inviscid modes propagate with the phase speed close to the flow velocity in the inflexion point.

Disappearance of the angle dependence of dispersion (here considered as the $\Re(\alpha(\beta))$ only) does not only play an important role at the late weakly nonlinear stages of transition, where some resonant interactions between 2D and 3D instability waves occur but also for the design of an active wave cancellation system. 


\section{Chapter 3}

\section{Demands on the active wave cancellation system}

The principles described in this chapter have been recognized partially during different stages of this investigation. They will be summarized here to facilitate the interpretation of following results.

In general, the demands on an active wave cancellation system obviously depend on the properties of the specific boundary layer and its dispersion characteristics, as well as on the nature of the external perturbations and the receptivity of the boundary layer to them. Furthermore, they will be influenced by the desired cancellation result and the complexity of the control system, that can be afforded. Therefore the present chapter will aim at giving an overview of the different parameters that are important to such a system and at some specific insights into the control of 3D disturbances rather than to present a complete set of design guidelines to an active wave cancellation system.

In order to develop the principles, it is appropriate to start with the general form of a multiple-sensor-actuator-sensor cancellation system on a flat plate as shown in Fig. 3.1. The flow from the left forms a boundary layer on the plate which is perturbed by external excitations, e.g. sound and free-stream turbulence, as outlined in chapter 2.1. Hence, a bandlimited disturbance wave field in the boundary layer is generated which is deformed and amplified by the dispersion characteristics of the Tollmien-Schlichting instability while propagating downstream (see Chapter 2.2). A spanwise array of reference sensors samples the incident wave field. The obtained signals are fed into a digital controller which models the propagation of the disturbances by means of digital filters. The digital filters are adapted by decorrelating the reference and error sensor signals. The output driving the actuators is computed such that the wave cancellation is achieved simultaneously for all the oblique waves contained in the incident disturbance wave field. This results in a minimization of the energy of the wave field at the 


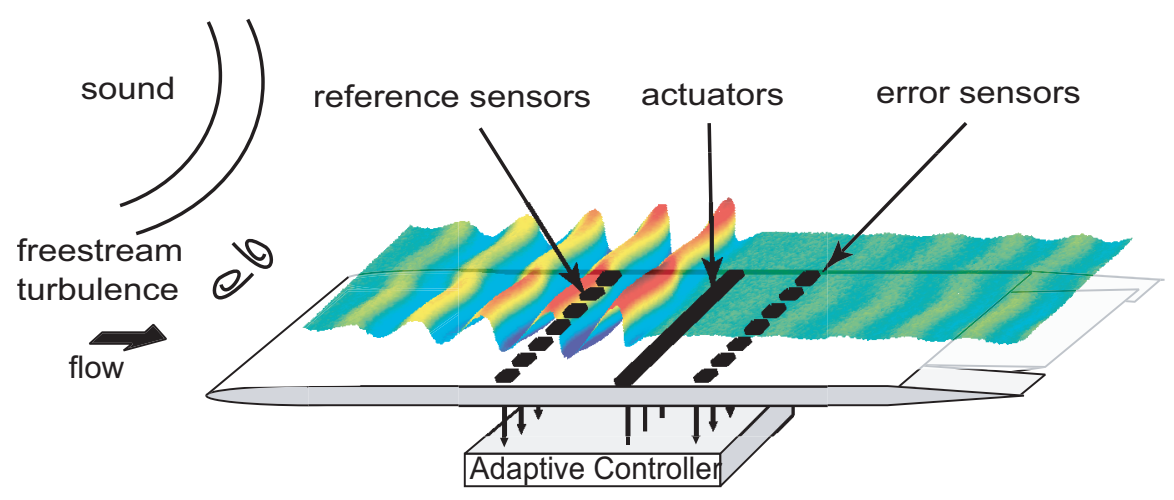

Figure 3.1: The schematic sketch of the cancellation device.

downstream array of error sensors. To work out the demands to such a control system, it is reasonable to start with the disturbance wave field under consideration.

\subsection{The influence of the receptivity and the dis- persion characteristics on the design of a wave cancellation system}

The key of determining the demands on a specific active wave cancellation system lies in the knowledge about the incident wave field the system is exposed to, and in the dispersion characteristics of the boundary layer.

The influence of the receptivity on the active wave cancellation system can obviously be divided into two aspects.

First, the frequency and wavenumber dependence of the transformation of the external perturbation into the disturbance wave field is of interest.

Second, the receptivity of the disturbances artificially introduced by means of the actuators is obviously of great interest in order to optimize the efficiency of the actuators.

Once the external perturbations of whatever origin are transformed to TS-waves, the spatial development is governed by the dispersion characteristics of the specific boundary layer. As outlined above, the dispersion relation depends on a variety of parameters, e.g. frequency, spanwise wavenumber, Reynolds number, streamwise and spanwise pressure gradients. 
3.1 The influence of the receptivity and the dispersion characteristics on the design of a wave cancellation system

The spatial and temporal correlation of the resulting wave field plays an important role in the active wave cancellation.

How does the spanwise correlation function of the disturbance wave field depend on the dispersion?

In order to give an answer to this question, a boundary layer on a flat plate with the streamwise and spanwise coordinates $x$ and $z$, respectively, is considered. Assuming that disturbances $\tau\left(x_{1}, z, t\right)$ are generated at a streamwise position $x_{1}$, e.g. at the leading edge $x_{1}=0$, their spanwise correlation can be written as

$$
R_{\tilde{\tau} \tilde{\tau}}\left(x_{1}, \Delta z, \omega\right)=\left\langle\tilde{\tau}\left(x_{1}, z, \omega\right) \tilde{\tau}\left(x_{1}, z+\Delta z, \omega\right)^{*}\right\rangle,
$$

where $\tilde{\tau}=\mathcal{F}_{t}\{\tau\}$ denotes here the temporal Fourier transform.

In the following, the incident disturbance distribution is considered in the wavenumber-frequency domain for streamwise position $x_{1}$

$$
T\left(x_{1}, \beta, \omega\right)=\mathcal{F}_{z, t}\left\{\tau\left(x_{1}, z, t\right)\right\} .
$$

The propagation to the streamwise location $x_{2}$ can be described as

$$
T\left(x_{2}, \beta, \omega\right)=T\left(x_{1}, \beta, \omega\right) e^{i \int_{x_{1}}^{x_{2}} \alpha(\beta, \omega, x) d x} .
$$

Hence, the streamwise development of the spanwise correlation can be written as

$$
\begin{aligned}
R_{\tilde{\tau} \tilde{\tau}}\left(x_{2}, \Delta z, \omega\right) & =\mathcal{F}_{\beta}^{-1}\left\{\overline{\left|T\left(x_{1}, \beta, \omega\right)\right|^{2}} e^{2 \Im\left\{\int_{x_{1}}^{x_{2}} \alpha(\beta, \omega) d x\right\}}\right\} \\
& =R_{\tilde{\tau} \tilde{\tau}}\left(x_{1}, \Delta z, \omega\right) * \mathcal{F}_{\beta}^{-1}\left\{e^{-2 \Im\left\{\int_{x_{1}}^{x_{2}} \alpha(\beta, \omega) d x\right\}}\right\} .
\end{aligned}
$$

Therefore the streamwise development of the spanwise correlation of the disturbance wave field can be obtained by the convolution of the incident spanwise correlation function by the inverse Fourier transform of the growth function which depends on Reynolds number, frequency, and spanwise wavenumber, respectively. This spanwise correlation function determines the optimum spanwise spacing of the sensors and the actuators as pointed out later.

Considering the streamwise transfer function in the $(\beta, \omega)$-plane, the next question which may arise is: What are the properties of this transfer function and which influences play a role? As already applied to equation (3.3), the streamwise propagation of the wave field between two downstream planes $x_{1}$ and $x_{2}$ can be described by the transfer function

$$
A(\beta, \omega)=e^{i \int_{x_{1}}^{x_{2}} \alpha(\beta, \omega, x) d x}
$$

Obviously, $A(\beta, \omega)$ will approximate 1 and the influence of the dispersion will decrease when the distance $x_{2}-x_{1}$ between these planes is decreased. This particularly means 
that the relation between the various $\beta$-modes does not change very much on their way from $x_{1}$ to $x_{2}$ or in other words $A(\beta, \omega)$ is sufficiently described by its values at a few points in the $(\beta, \omega)$-plane which also means that the spatial structure of the wave field is nearly preserved. This may be described by the fact that the flat transfer function $A(\omega, \beta)$ has an inverse Fourier transform $\tilde{A}(\Delta z, \omega)=\mathcal{F}_{\beta}^{-1}\{A\}$ that is practically zero outside a narrow range $|\Delta z|<\Delta z^{(A)}$. Therefore, in the prediction of the wave field at the streamwise position $x_{2}$

$$
\tilde{\tau}\left(x_{2}, z, \omega\right)=\tilde{\tau}\left(x_{1}, z, \omega\right) * \tilde{A}(\Delta z, \omega)
$$

only the narrow spanwise range $z \pm \Delta z^{(A)}$ has to be considered.

How could a possible control system look like?

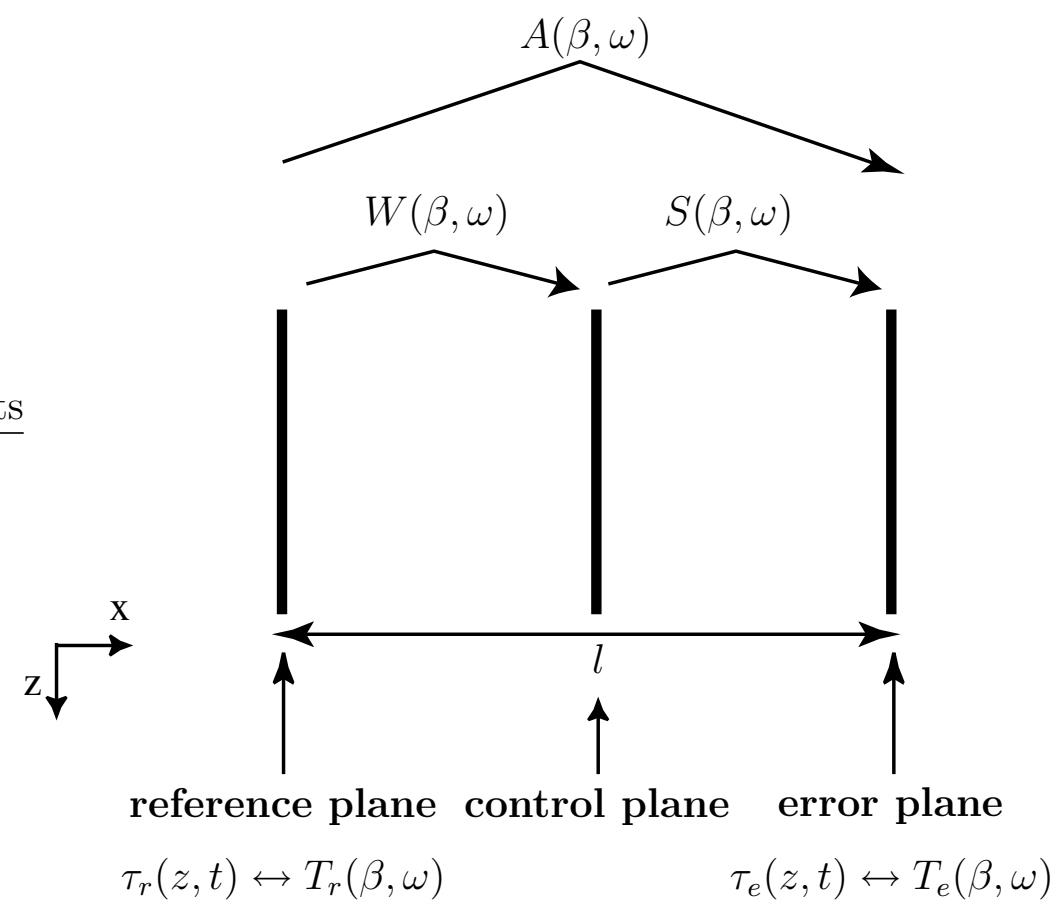

Figure 3.2: Control strategy in terms of continuous temporal-spatial spectra. In the following, the quantities at the streamwise location of the reference plane are denoted by an index $r$, similarly for the error plane by an index $e$.

Fig. 3.2 shows such a control system, where three spanwise planes are considered: the reference plane, the control plane, and the error plane. Assuming that the continuous spectra at the reference plane $T_{r}(\beta, \omega)$ and the error plane $T_{e}(\beta, \omega)$ are known, the transfer function between these planes is obtained as $\tilde{A}(\beta, \omega)=e^{i \int_{x_{r}}^{x_{e}} \alpha(\beta, \omega, x) d x}$. Furthermore, assuming that a continuous spectrum can be generated at the control plane such 
3.1 The influence of the receptivity and the dispersion characteristics on the design of a wave cancellation system

that the spectrum $T_{e}(\beta, \omega)$ at the error plane is cancelled, the transfer function $S(\beta, \omega)$ has to be taken into account. The way of generating such a control spectrum is one of the central objective of the present investigation. Generally speaking, the control spectrum is computed from $T_{r}(\beta, \omega)$ by multiplication with the transfer function $W(\beta, \omega)$ between the reference plane and the control plane, which to find experimentally is an essential task in present study. The optimum transfer function fulfills the condition:

$$
W(\beta, \omega) \stackrel{!}{=}-\frac{A(\beta, \omega)}{S(\beta, \omega)}
$$

As pointed out previously, $W(\beta, \omega)$ can be reduced to simple form by decreasing the streamwise distance between reference and the error plane. The possibility of minimizing this distance in a real system however is limited by a constraint that will be explained later. Thus, the question may be asked, if there are further possibilities to achieve this simplification. One way to accomplish this, may be to design the physical part of the control system such that $S(\beta, \omega)$ becomes as similar to $A(\beta, \omega)$ as possible.

The practical implementation of a control system needs a spatially and temporally discrete formulation of the relations that have been described with continuous variables in the preceding paragraph. This is possible according to the sampling theorem, since the considered wave fields are bandlimited with respect to space and time.

Due to the stochastic nature of these wave fields their spatial structure can be characterized either by its spanwise wavenumber spectrum or by its spanwise correlation both of which depend on the temporal frequency. The maximum spanwise wavenumber $\Delta \beta_{\max }$ found in the wavenumber spectrum and the extent $\Delta z_{\max }$ of the spanwise correlation function determine the number $N_{\max }$ of degrees of freedom in the wave field as

$$
N_{\max }=\frac{1}{\pi} \Delta \beta_{\max } \Delta z_{\max }
$$

Herein, $N_{\max }$ obviously depends on the definition of $\Delta \beta_{\max }$ and $\Delta z_{\max }$, i.e. on the values to which the wavenumber spectrum and the correlation function have to drop at $\beta=\Delta \beta_{\max }$ and $\Delta z=\Delta z_{\max }$, respectively. The optimum cancellation wave field should have equal amplitude as the wave field to be cancelled but counter-phase and therefore contains the same number of degrees of freedom. Then the accuracy of cancellation will depend on the definition of $\Delta \beta_{\max }$ and $\Delta z_{\max }$ provided this optimum wave field can be generated by the control system.

The following sections deal with the derivation of a discrete control system that is able to model the transfer function $W(\beta, \omega)$. 


\subsection{The optimum linear model of the system}

In order to develop an optimum linear model of the system achieving an optimum cancellation of the wave field, one may preliminarily switch to a different way of describing the propagation of the wave field, namely in terms of point-to-point transfer functions between sensor signals and signals driving the actuators.

Starting with a sensor-actuator configuration as sketched in Fig. 3.3, the wave field is sampled by an array of $J$ reference sensors; the obtained signals are filtered by the controller to drive an array of $K$ actuators generating a counter-phase wave field to minimize the signals measured by $M$ error sensors. This can be achieved in an optimum sense by minimizing all correlations between the reference and error sensors.



Figure 3.3: A general diagram of a sensor-actuator-sensor configuration and its notation, here with equal spanwise spacing for its components. The configuration with $J=K=M$ will be referred to as symmetric configuration.

To find the optimum settings of the controller, one may start with the signal flow diagram shown in Fig. 3.4. The superposition of the incident wave field and the cancellation wave field, measured at the error sensors, is considered first. The $M$ error signals can be expressed as the sum of both components,

$$
\mathbf{e}=\mathbf{d}+\mathbf{y}
$$

where $\mathbf{d}^{1}$ denotes the original wave field, which is intended to be cancelled by the actuator wave field $\mathbf{y}$. In equation (3.9) the signals are denoted in the frequency domain

\footnotetext{
${ }^{1}$ The common notation " $d "$ means desired and stems from the aim that the desired output $y$ of the cancellation system equals $-d$ in order to achieve perfect cancellation.
} 


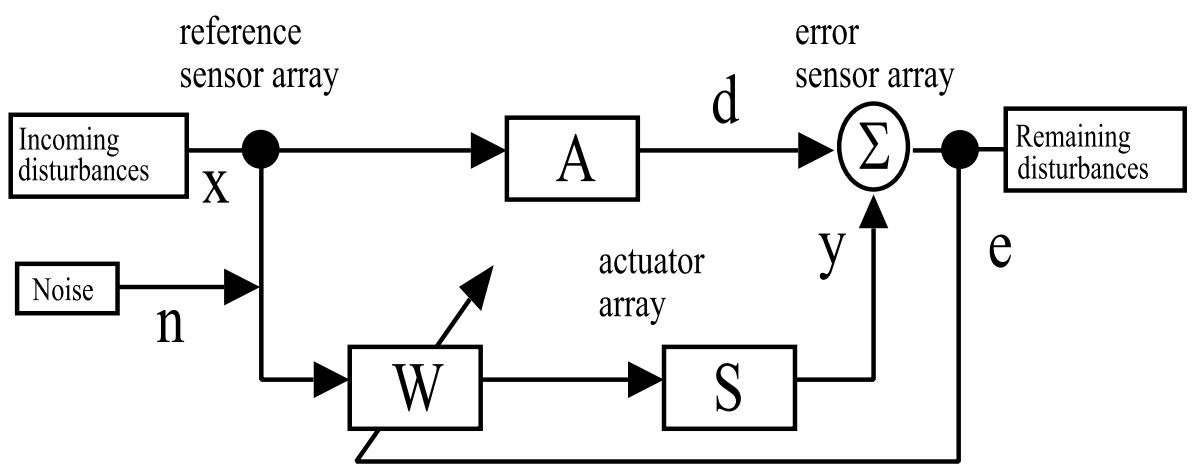

Figure 3.4: Signal flow diagram of the system in terms of transfer matrices

such that the $M$ Fourier transforms of the signals are comprised in a vector, e.g.

$$
\mathbf{e}=\left(e_{1}(\omega), e_{2}(\omega), e_{3}(\omega), \ldots, e_{M}(\omega)\right)^{T} .
$$

Expressing $\mathbf{d}$ and $\mathbf{y}$ in equation (3.9) in terms of transfer functions as shown in Fig. 3.4 leads to

$$
\mathbf{e}=\mathbf{A x}+\mathbf{S W}(\mathbf{x}+\mathbf{n})
$$

The complex transfer functions between the $J$ reference sensors and the $M$ error sensors are comprised in the matrix

$$
\mathbf{A}=\left[\begin{array}{cccc}
A_{11}(\omega) & A_{12}(\omega) & \cdots & A_{1 J}(\omega) \\
A_{21}(\omega) & A_{22}(\omega) & \cdots & A_{2 J}(\omega) \\
\vdots & \vdots & \ddots & \vdots \\
A_{M 1}(\omega) & A_{M 2}(\omega) & \cdots & A_{M J}(\omega)
\end{array}\right]
$$

The $J$ measured reference signals, which are usually composed of the "ideal signal"

$$
\mathbf{x}=\left(x_{1}(\omega), x_{2}(\omega), x_{3}(\omega), \ldots, x_{J}(\omega)\right)^{T}
$$

and some uncorrelated noise $\mathbf{n},\left(\left\langle\mathbf{n} \mathbf{x}^{\mathbf{H}}\right\rangle=0\right)$, are filtered by the transfer matrix of the controller

$$
\mathbf{W}=\left[\begin{array}{cccc}
W_{11}(\omega) & W_{12}(\omega) & \cdots & W_{1 J}(\omega) \\
W_{21}(\omega) & W_{22}(\omega) & \cdots & W_{2 J}(\omega) \\
\vdots & \vdots & \ddots & \vdots \\
W_{K 1}(\omega) & W_{K 2}(\omega) & \cdots & W_{K J}(\omega)
\end{array}\right]
$$


to drive the array of $K$ actuators. Herein, $W_{k j}(\omega)$ denotes the transfer function from the $j$ 'th reference sensor to the $k$ 'th actuator.

The special case of equal equidistant spacing between the elements of the three arrays of sensors and actuators, respectively, and for streamwise alignment of "channels", each consisting of a reference sensor, an actuator, and an error sensor, is denoted as symmetric configuration, below. In this case the diagonal elements of $\mathbf{W}$ will describe the streamwise transfer functions and the off-diagonal elements represent the connections in the oblique directions. This applies also to the matrices $\mathbf{A}$ and $\mathbf{S}$.

$\mathbf{S}$ represents the transfer matrix from the $K$ actuators to the $M$ error sensors,

$$
\mathbf{S}=\left[\begin{array}{cccc}
S_{11}(\omega) & S_{12}(\omega) & \cdots & S_{1 K}(\omega) \\
S_{21}(\omega) & S_{22}(\omega) & \cdots & S_{2 K}(\omega) \\
\vdots & \vdots & \ddots & \vdots \\
S_{M 1}(\omega) & S_{M 2}(\omega) & \cdots & S_{M K}(\omega)
\end{array}\right]
$$

According to the theory of Wiener filters the optimum filter settings $\mathbf{W}_{\text {opt }}$ cancel the correlations between the reference signals $\mathbf{x}+\mathbf{n}$ and the remaining error signals $\mathbf{e}$.

$$
\begin{aligned}
0 & \stackrel{!}{=}\left\langle\mathbf{e}(\mathbf{x}+\mathbf{n})^{\mathbf{H}}\right\rangle \\
& =\left\langle(\mathbf{d}+\mathbf{y})(\mathbf{x}+\mathbf{n})^{\mathbf{H}}\right\rangle \\
& =\mathbf{A}\left\langle\mathbf{x}(\mathbf{x}+\mathbf{n})^{\mathbf{H}}\right\rangle+\mathbf{S} \mathbf{W}_{\mathbf{~ o p t}}\left\langle(\mathbf{x}+\mathbf{n})(\mathbf{x}+\mathbf{n})^{\mathbf{H}}\right\rangle \\
& =\mathbf{P}+\mathbf{S} \mathbf{W}_{\text {opt }} \mathbf{R} .
\end{aligned}
$$

Herein, the correlations between the $J$ signals of the reference sensors $\left\langle(\mathbf{x}+\mathbf{n})(\mathbf{x}+\mathbf{n})^{\mathbf{H}}\right\rangle$ are comprised in the matrix

$$
\mathbf{R}=\left[\begin{array}{cccc}
R_{11}(\omega) & R_{12}(\omega) & \cdots & R_{1 J}(\omega) \\
R_{21}(\omega) & R_{22}(\omega) & \cdots & R_{2 J}(\omega) \\
\vdots & \vdots & \ddots & \vdots \\
R_{J 1}(\omega) & R_{J 2}(\omega) & \cdots & R_{J J}(\omega)
\end{array}\right]
$$

and the cross-correlations between reference and error sensors $\mathbf{A}\left\langle\mathbf{x} \mathbf{x}^{\mathbf{H}}\right\rangle$ are contained in the matrix

$$
\mathbf{P}=\left[\begin{array}{cccc}
P_{11}(\omega) & P_{12}(\omega) & \cdots & P_{1 M}(\omega) \\
P_{21}(\omega) & P_{22}(\omega) & \cdots & P_{2 M}(\omega) \\
\vdots & \vdots & \ddots & \vdots \\
P_{J 1}(\omega) & P_{J 2}(\omega) & \cdots & P_{J M}(\omega)
\end{array}\right]
$$


Hence, provided the matrices $\mathbf{S}$ and $\mathbf{R}$ are invertible, the optimum solution for the filter transfer functions is given by the (causally unconstrained) Wiener-filter

$$
\mathbf{W}_{\text {opt }}=-\mathbf{S}^{-1} \mathbf{P} \mathbf{R}^{-1}
$$

By eliminating the correlation between the reference and the error signals, this filter minimizes the remaining disturbances. This filter is causally unconstrained because its inverse Fourier transform may contain components at negative time. As pointed out below, only the part at times greater than zero can be considered properly by the control system and solely if the rest is zero.

In the case of the symmetric configuration (see Fig.3.3) the term $\mathbf{P} \mathbf{R}^{-\mathbf{1}}$ models the transfer matrix between the reference sensors and the error sensor, corresponding to the transfer function $A(\beta, \omega)$ in the previous section. Thus, the diagonal elements of $\mathbf{W}_{\text {opt }}$ describe the filters in streamwise direction while the off-diagonal elements represent the connections in oblique directions. Apart from edge effects, the dispersion relation of oblique TS-waves, the streamwise extension of the cancellation device as well as the necessary spatial filtering of the actuators affect these transfer functions and correlation functions. Due to the Toeplitz-form of the related matrices, the streamwise channels (the diagonal elements of the matrices) consisting each of a reference sensor, an actuator and an error sensor (arranged in the streamwise direction) are the most important components of the cancellation system, and, in fact, they are the only needed components if pure 2D TS-waves were to be eliminated.

Summarizing, the optimum setting of a MIMO-filter has been derived, that eliminates all correlations between two finite sets of sensor signals by driving a finite set of actuators which affect the error signals while the reference signals remain unaffected. In principle, no assumptions have been made concerning the spatial arrangement and homogeneity of the components.

Thus, the question arises how the description of the wave cancellation by means of discrete transfer functions and, on the other hand, by propagating modes are related to each other.

\subsection{The observability and controllability of the dis- turbance wave field}

In order to define the conditions which have to be satisfied to obtain observability and controllability of the disturbance wave field, it is necessary to refine the model derived above. Particularly, the mapping of the continuous spectrum of modes, in terms of oblique wavenumbers, to the discrete representation by the controller has to be worked 
out. This discretization should be carried out such that parts of the spectrum which propagate similarly are clustered to a single transfer function.

As pointed out previously, the disturbance wave field is confined to a wavenumber range and a spanwise correlation length and can therefore be composed by a finite number of discrete modes. The number of degrees of freedom $N_{\max }$ are determined by both the maximum spanwise correlation length and the maximum spanwise wavenumber spectrum (see equation (3.8)). Thus, the optimum control system must be able to model $N_{\max }$ independent modes in the worst case. As pointed out in section 3.1 it should be possible however to further reduce the number of modes that have to be treated independently. In order to achieve a complete discrete representation of the continuous bandlimited mode spectrum, the spatial Nyquist-theorem has to be fulfilled. Hereby, the maximum spanwise spacing $\Delta z_{N y}$ of the device components, e.g. the reference sensors, is given by

$$
\Delta z_{N y} \stackrel{!}{\leq} \frac{\pi}{\beta_{\max }}=\frac{\Delta z_{\max }}{N_{\max }}
$$

In the case of a large symmetric configuration $\left(J=K=M \gg N_{\max }\right.$, neglecting edge-effects), the amplitudes of the $N=N_{\max }$ modes contained in the incident wave field are estimated from the $J$ reference signals. The following mappings of the complex mode amplitudes $\boldsymbol{\Phi}_{\mathbf{x}}$ at the reference sensor array (see Fig. 3.5) can be noted:

$$
\mathbf{x}=\mathbf{H}_{\mathbf{x}} \boldsymbol{\Phi}_{\mathbf{x}}, \quad \mathbf{H}_{\mathbf{x}} \in \mathbb{C}^{(J \times N)}
$$

and $\boldsymbol{\Phi}_{\mathbf{e}}$ at the error sensor array:

$$
\mathbf{e}=\mathbf{H}_{\mathbf{e}} \boldsymbol{\Phi}_{\mathbf{e}} \quad \mathbf{H}_{\mathbf{e}} \in \mathbb{C}^{(M \times N)} .
$$

The explicit dependence on $\omega$ has been dropped for notational convenience. The matrices $\mathbf{H}_{\mathbf{x}}$ and $\mathbf{H}_{\mathbf{e}}$ represent the sensitivity of the sensor arrays to the specific modes and may vary with frequency and spanwise wavenumber. Similarly, a mapping can be expressed for the actuator array generating a mode spectrum $\mathbf{\Phi}_{\mathbf{y}}$, driven by $K$ output signals $\mathbf{y}$ :

$$
\boldsymbol{\Phi}_{\mathbf{y}}=\mathbf{H}_{\mathbf{y}} \mathbf{y} \quad \mathbf{H}_{\mathbf{y}} \in \mathbb{C}^{(N \times K)} .
$$

Following the same Ansatz as in equation (3.11)

$$
\begin{aligned}
\mathbf{e} & =\mathbf{H}_{\mathbf{e}}\left\{\mathbf{A}_{\boldsymbol{\Phi}} \boldsymbol{\Phi}_{\mathbf{x}}+\mathbf{S}_{\boldsymbol{\Phi}} \boldsymbol{\Phi}_{\mathbf{y}}\right\} \\
& =\mathbf{H}_{\mathbf{e}}\left\{\mathbf{A}_{\boldsymbol{\Phi}} \boldsymbol{\Phi}_{\mathbf{x}}+\mathbf{S}_{\boldsymbol{\Phi}} \mathbf{H}_{\mathbf{y}} \mathbf{W}\left(\mathbf{H}_{\mathbf{x}} \boldsymbol{\Phi}_{\mathbf{x}}+\mathbf{n}\right)\right\}
\end{aligned}
$$

is obtained for the error signals and

$$
\hat{\mathbf{x}}=\left(\mathbf{H}_{\mathbf{x}} \boldsymbol{\Phi}_{\mathbf{x}}+\mathbf{n}\right)
$$




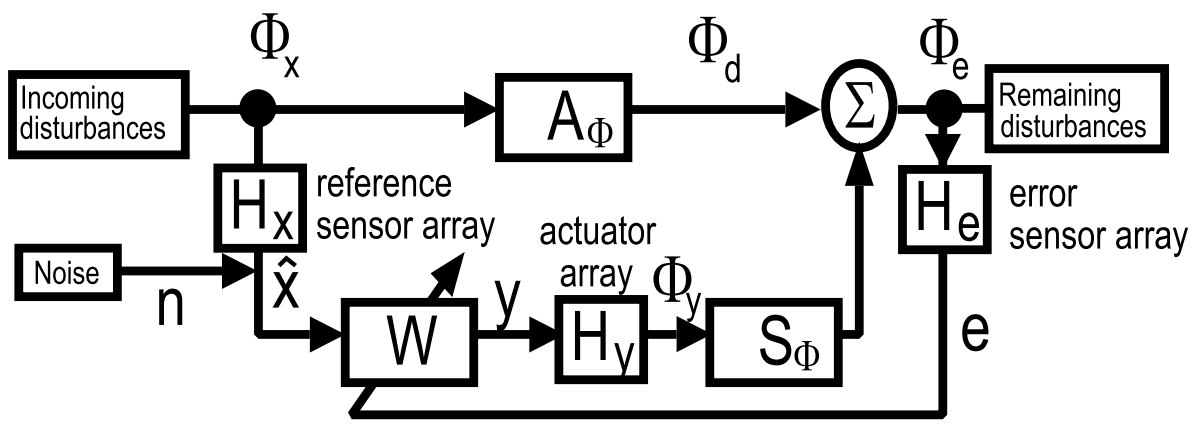

Figure 3.5: Refinement of the signal flow diagram of the system in terms of transfer matrices.

for the reference signals. The optimum filter can again be determined by the vanishing correlation between both signals:

$$
\begin{aligned}
0 & \stackrel{!}{=}\left\langle\mathbf{e} \hat{\mathbf{x}}^{\mathbf{H}}\right\rangle \\
& =\left\langle\mathbf{H}_{\mathbf{e}}\left\{\mathbf{A}_{\boldsymbol{\Phi}} \boldsymbol{\Phi}_{\mathbf{x}}+\mathbf{S}_{\boldsymbol{\Phi}} \mathbf{H}_{\mathbf{y}} \mathbf{W}_{\mathbf{o p t}}\left(\mathbf{H}_{\mathbf{x}} \boldsymbol{\Phi}_{\mathbf{x}}+\mathbf{n}\right)\right\}\left(\mathbf{H}_{\mathbf{x}} \boldsymbol{\Phi}_{\mathbf{x}}+\mathbf{n}\right)^{\mathbf{H}}\right\rangle \\
& =\mathbf{H}_{\mathbf{e}} \underbrace{\mathbf{A}_{\boldsymbol{\Phi}}\left\langle\boldsymbol{\Phi}_{\mathbf{x}} \boldsymbol{\Phi}_{\mathbf{x}}^{\mathbf{H}}\right\rangle}_{\equiv \mathbf{P}_{\boldsymbol{\Phi}}} \mathbf{H}_{\mathbf{x}}^{\mathbf{H}}+\mathbf{H}_{\mathbf{e}} \mathbf{S}_{\boldsymbol{\Phi}} \mathbf{H}_{\mathbf{y}} \mathbf{W}_{\mathbf{o p t}} \underbrace{\left\langle\left(\mathbf{H}_{\mathbf{x}} \boldsymbol{\Phi}_{\mathbf{x}}+\mathbf{n}\right)\left(\mathbf{H}_{\mathbf{x}} \boldsymbol{\Phi}_{\mathbf{x}}+\mathbf{n}\right)^{\mathbf{H}}\right\rangle}_{\equiv \mathbf{R}_{\boldsymbol{\Phi}}} \\
& =\mathbf{H}_{\mathbf{e}}\left\{\mathbf{P}_{\boldsymbol{\Phi}} \mathbf{H}_{\mathbf{x}}^{\mathbf{H}}+\mathbf{S}_{\boldsymbol{\Phi}} \mathbf{H}_{\mathbf{y}} \mathbf{W}_{\mathbf{o p t}} \mathbf{R}_{\boldsymbol{\Phi}}\right\}
\end{aligned}
$$

If the matrices $\mathbf{H}_{\mathbf{y}}, \mathbf{S}_{\boldsymbol{\Phi}}$ and $\mathbf{R}_{\boldsymbol{\Phi}}$ are invertible, the elimination of all correlations between reference and error signals is achieved by the optimum filter settings:

$$
\mathrm{W}_{\mathrm{opt}}=-\mathrm{H}_{\mathrm{y}}^{-1} \mathbf{S}_{\Phi}^{-1} \mathbf{P}_{\Phi} \mathbf{H}_{\mathrm{x}}^{\mathrm{H}} \mathbf{R}_{\Phi}^{-1}
$$

By neglecting the noise component $\mathbf{n}$ the matrix $\mathbf{R}_{\boldsymbol{\Phi}}$ can be rewritten as

$$
\begin{aligned}
\mathbf{R}_{\Phi} & =\left\langle\left(\mathbf{H}_{\mathbf{x}} \boldsymbol{\Phi}_{\mathbf{x}}+\mathbf{n}\right)\left(\mathbf{H}_{\mathbf{x}} \boldsymbol{\Phi}_{\mathbf{x}}+\mathbf{n}\right)^{\mathbf{H}}\right\rangle \\
& \approx \mathbf{H}_{\mathbf{x}}\left\langle\boldsymbol{\Phi}_{\mathbf{x}} \boldsymbol{\Phi}_{\mathbf{x}}^{\mathbf{H}}\right\rangle \mathbf{H}_{\mathbf{x}}^{\mathbf{H}}
\end{aligned}
$$

Assuming that the modes are independent of each other, which generally will not be the case, the matrix $\left\langle\boldsymbol{\Phi}_{\mathbf{x}} \boldsymbol{\Phi}_{\mathbf{x}}^{\mathbf{H}}\right\rangle$ will become a diagonal matrix with full rank. By rewriting equation (3.26)

$$
-\underbrace{H_{\mathbf{e}} A_{\Phi}\left\langle\Phi_{\mathrm{x}} \Phi_{\mathrm{x}}^{\mathrm{H}}\right\rangle \mathbf{H}_{\mathrm{x}}^{\mathrm{H}}}_{\equiv P}=\underbrace{\mathbf{H}_{\mathrm{e}} \mathrm{S}_{\boldsymbol{\Phi}} \mathbf{H}_{\mathrm{y}}}_{\equiv \mathrm{S}} \mathbf{W}_{\mathrm{opt}} \underbrace{\mathbf{H}_{\mathbf{x}}\left\langle\Phi_{\mathrm{x}} \Phi_{\mathrm{x}}^{\mathbf{H}}\right\rangle \mathbf{H}_{\mathrm{x}}^{\mathrm{H}}}_{\equiv \mathbf{R}}
$$

it becomes obvious that the right-side division by the matrix $\mathbf{R}$ can be carried out if the row-vectors of the matrices $\mathbf{R}$ and $\mathbf{P}$ span the same subspace $\in \mathbb{R}^{N}$. In order 
to satisfy this condition the sensitivity of the sensor arrays $\mathbf{H}_{\mathbf{x}}$ and $\mathbf{H}_{\mathbf{e}}$ to the modes should be equal. Therefore it is reasonable to choose the same spacing and the same orientation of the sensors for both sensor arrays.

The case of a symmetric system, $J=M=2$ is considered, where the reference sensors are placed at $x=0, z= \pm \frac{d}{2}$ and the errors sensors at $x=l, z= \pm \frac{d}{2}$, where $d=\Delta z_{r}=\Delta z_{e}$ and $l=\Delta x_{r a}+\Delta x_{a e}$. Let the signal component $\Psi(x, z)$ be caused by the $n$ 'th mode to be characterized by a spanwise wavenumber $\beta$ with the corresponding streamwise wavenumber $\alpha(\beta)$ :

$$
\Psi_{n}(x, z)=\Phi_{0}\left(\beta_{n}\right) e^{i\left(\alpha\left(\beta_{n}\right) x+\beta_{n} z\right)},
$$

where $\Phi_{0}\left(\beta_{n}\right)$ denotes the initial modal amplitude of the $n$ 'th mode. Assuming that the sensors have the same sensitivity to each mode, the measured signals can be written as

$$
\begin{aligned}
& x_{1}=\sum_{n=1}^{N} \Phi_{0}\left(\beta_{n}\right) e^{i \beta_{n} \frac{d}{2}} \\
& x_{2}=\sum_{n=1}^{N} \Phi_{0}\left(\beta_{n}\right) e^{-i \beta_{n} \frac{d}{2}} \\
& e_{1}=\sum_{n=1}^{N} \Phi_{0}\left(\beta_{n}\right) e^{i\left(\alpha\left(\beta_{n}\right) l+\beta_{n} \frac{d}{2}\right)} \\
& e_{2}=\sum_{n=1}^{N} \Phi_{0}\left(\beta_{n}\right) e^{i\left(\alpha\left(\beta_{n}\right) l-\beta_{n} \frac{d}{2}\right)}
\end{aligned}
$$

Therefore the matrices $\mathbf{H}_{\mathbf{x}}$ and $\mathbf{H}_{\mathbf{e}}$ can be written as

$$
\mathbf{H}_{\mathbf{x}}=\left[\begin{array}{cccc}
e^{i \beta_{1} \frac{d}{2}} & e^{i \beta_{2} \frac{d}{2}} & \cdots & e^{i \beta_{N} \frac{d}{2}} \\
e^{-i \beta_{1} \frac{d}{2}} & e^{-i \beta_{2} \frac{d}{2}} & \cdots & e^{-i \beta_{N} \frac{d}{2}}
\end{array}\right]
$$

and

$$
\mathbf{H}_{\mathbf{e}}=\mathbf{H}_{\mathbf{x}}\left[\begin{array}{cccc}
e^{i \alpha\left(\beta_{1}\right) l} & 0 & \cdots & 0 \\
0 & e^{i \alpha\left(\beta_{2}\right) l} & \ddots & \vdots \\
\vdots & \ddots & \ddots & 0 \\
0 & \cdots & 0 & e^{i \alpha\left(\beta_{N}\right) l}
\end{array}\right]
$$

Hence, the assumption that the matrices $\mathbf{R}$ and $\mathbf{P}$ span the same subspace $\in \mathbb{R}^{N}$ is satisfied for the case where all $\alpha\left(\beta_{n}\right)$ are equal, i.e. no dispersion exists, or if the condition $\alpha\left(\beta_{n}\right) l \ll 1$ is satisfied, which means that the streamwise distance between 
the sensor arrays is negligible. Furthermore, it is also conceivable that the neglected noise component $\mathbf{n}$ can additionally endanger this condition by either hiding modes at the reference sensors which become visible at the error sensors due to amplification, or by spoiling of the estimates.

\subsubsection{Observability}

As pointed out earlier, the main precondition to be satisfied, in order to guarantee the observability of all modes present in the disturbance wave field, is the spatial sampling theorem. Therefore, the maximum permissible lateral spacing of the sensors is given by half the minimum lateral wavelenght found in the wavenumber spectrum, the Fourier transform of the lateral correlation function. This determines $\beta_{N y}$, the so-called Nyquist wavenumber of the sensor array. Fulfilling this precondition assures that no aliasing effects occur in the mappings from the disturbance wave field to the obtained signals, see equations (3.21), (3.22). The matrices $\mathbf{H}_{\mathbf{x}}$ and $\mathbf{H}_{\mathbf{e}}$ must not become singular for any frequency in the relevant frequency range. If entries in the matrix $\mathbf{H}_{\mathbf{e}}$ become close to zero for a specific frequency, the control system will not observe the wave field at this frequency, which can obviously decrease the performance of the system drastically, see equation (3.26). If the entries in the matrix $\mathbf{H}_{\mathbf{x}}$ become small for any frequency, the resulting $\mathbf{W}_{\text {opt }}$ becomes large if the obtained error signals are non-zero, see equation (3.27). If this is the case one has to take precautions by using an adaptation algorithm limiting the control power, but nevertheless the performance will be poor for this frequency.

\subsubsection{Controllability}

The controllability of the disturbance wave field is determined by the condition of the matrix $\mathbf{S}=\mathbf{H}_{\mathbf{e}} \mathbf{S}_{\boldsymbol{\Phi}} \mathbf{H}_{\mathbf{y}}$, i.e. that is invertible. Considering the simple case of a symmetric system with $J=K=M=2$ under symmetric conditions, the matrix

$$
\mathbf{S}=\left[\begin{array}{ll}
S_{11} & S_{12} \\
S_{21} & S_{22}
\end{array}\right]
$$

is invertible, if the streamwise components are not equal to the diagonal elements: $\underbrace{S_{11}=S_{22}}_{\equiv S_{d}} \neq \underbrace{S_{12}=S_{21}}_{\equiv S_{n d}}$. Hence, the inverse of $\mathbf{S}$ is simply obtained by

$$
\left[\begin{array}{cc}
S_{d} & S_{n d} \\
S_{n d} & S_{d}
\end{array}\right]^{-1}=\frac{1}{S_{d}^{2}-S_{n d}^{2}}\left[\begin{array}{cc}
S_{d} & -S_{n d} \\
-S_{n d} & S_{d}
\end{array}\right]
$$


Thus, the difference between the diagonal and off-diagonal elements determines the invertibility of the considered transfer matrices and therefore depends on the streamwise distance $\Delta x_{a e}$ and the spanwise distance $\Delta z_{e}$ (as defined in Fig. 3.3).

In order to avoid also aliasing-effects when the wave field at the location of the error sensors is sampled, the actuators must not produce any components with wavenumbers beyond $\beta_{N y}$ of the error sensor array; in other words the spanwise distribution of the generated cancellation wave must be sufficiently smooth. This demand may be fulfilled either by the design of the actuators and/or by tailoring the relation between the signals that drive the actuators. Nonetheless the distribution of the generated cancelling disturbances within the sampled wavenumber range should be flat and zero elsewhere, to allow the cancellation of all sampled wavenumber components. Similar requirements have to be fulfilled for the time-frequency domain. Hence, the frequency distribution has to be designed adequately smooth within the relevant frequency range.

In order to obtain an accurate estimate of the remaining disturbances, the error sensors should not be placed to close to the actuators, i.e. in the near field, because evanescent modes could spoil the detected signals. Assuming a linear generation process, this condition can be satisfied quite easily due to the rather strong decay in streamwise direction of all modes $u(y)(\beta=0)$ with discrete eigenvalues, except of the least stable one. The kinetic energy of the other discrete modes $u(y)$ decreases by more than a factor of 1000 within the characteristic wavelength (Boiko, Grek, Dovgal, and Kozlov 2002).

\subsection{The constraint of causality}

Due to the random character of the disturbances to be controlled the constraint of causality applies to the control system ${ }^{2}$. The constraint imposes a control strategy to be causal, which means that the control system must not be expected to respond to a reference signal that has not yet been measured. In other words the response of the control system can not occur ahead of its excitation, and thus it guarantees that the system is physically realizable in the time-domain.

The minimum streamwise distance between the reference sensors and the actuators is determined by the maximum group velocity of the disturbances, which is for the Blasius boundary layer within a range of $c_{g r}=0.35 \cdots 0.45 \times U_{\infty}$, where $U_{\infty}$ denotes the free-stream velocity. Therefore, to fulfill the required causality the electric delay of the counter-phase signal, which is mainly caused by the necessary anti-aliasing and reconstruction lowpass filters, has to be smaller than the minimum time the disturbances

\footnotetext{
${ }^{2}$ Whereas in the case of harmonic disturbances, causality is not a constraint, since future signals are a repetition of past signals.
} 
need to travel from the reference sensors to the actuators.

\subsection{Consideration of the dispersion characteristics}

This section summarizes the results concerning the consideration of the dispersion by the control system.

The streamwise distance $\Delta x_{r a}$ between the reference sensors and the actuators determines the influence of the dispersion characteristics $\alpha(\omega, \beta, \operatorname{Re}(x))$. In fact, the dynamics of the part of the boundary layer between the reference sensor array and the array of actuators is described by the transfer function $e^{i \overline{\alpha(\omega, \beta, R e(x))} \Delta x_{r a}}$ wherein $\overline{\alpha(\omega, \beta, R e(x))}$ is an appropriate average of $\alpha(x)$ and is modelled by a network of digital filters as described by the matrix $\mathbf{W}$. The dependence on $\omega$ is accounted for by the impulse responses of the filters, and it can be expected that the length of these impulse responses increases with the distance $\Delta x_{r a}$. The dependence on $\beta$ affects the number of different modes that have to be treated by the filter network, and it can be anticipated that the connectivity of the network has to grow with increasing distance $\Delta x_{r a}$. In other words if $\alpha(\omega, \beta, \operatorname{Re}(x))$ could be considered to be independent of $\beta$, only one single streamwise transfer function would be sufficient to model the propagation of the TS-waves, i.e. the transfer matrices will degenerate to a single transfer function. Another way of achieving this simplification may be to introduce a spatial filtering, e.g. by the actuators, such that it has the same dependence on $\beta$ as the dispersion of the part of the boundary layer. The Toeplitz-form of the spatial transfer process that becomes obvious from the discussion carried out in section 3.1, may be exploited by algorithms to simplify the inversion of the relevant matrices.

\subsection{The real-time constraint to the controller}

The real-time constraint to the controller is mainly a limitation to the complexity of the system model, i.e. to the algorithms estimating the optimum set of transfer functions, which describe the propagation of the wave field. In order to get a proper analogdigital and digital-analog conversion of the signals the temporal sampling theorem has to be fulfilled, i.e. the sampling frequency has to be more than twice the highest relevant frequency component occurring in the signal. Therefore the sampling period determines the cycle time, within each sensor signal sample is recorded and the output signal sample for the actuators has to be computed. 


\section{Chapter 4}

\section{Adaptive signal processing methods}

In order to model the amplification and the propagation of the disturbances both of which depend on frequency and spanwise wavenumber, adaptive filters are applied to model the relevant transfer functions, as well as to compensate for the frequency responses of the sensors and the actuators and thereby for differences in the efficiency of the actuators and in the sensitivity of the sensors.

This chapter gives a short introduction to adaptive filters, followed by a summary of the system identification and active wave cancellation schemes. Particularly, the multi-input-multi-output system and its stability is discussed in detail at the end of the present chapter.

\subsection{Adaptive filters}

\subsubsection{Digital filters}

In every discrete time step $n$ a digital filter generates an output sample $y(n)$ from an input time series, in vector notation:

$$
\mathbf{x}[n]=(x[n], x[n-1], \ldots, x[n-N+1])^{\mathbf{T}} .
$$

A signal vector of this kind represents a digital signal, which is obtained from the original signal by periodic sampling at the times $n \cdot \Delta t . x[n]$ denotes the current sample and $x[n-i]$ the sample recorded $i$ time steps earlier. In general, linear, causal filters can be described by the equation:

$$
y[n]=\sum_{i=0}^{N-1} a_{i} x[n-i]+\sum_{j=1}^{M} b_{j} y[n-j],
$$


where the first sum describes the transversal part of the filter while the second sum determines the recursive part.

In order to obtain the transfer function $H(z)$ of the filter, the Z-transform is applied to equation (4.2), and after some rearrangement

$$
H(z)=\frac{Y(z)}{X(z)}=\frac{\sum_{i=0}^{N-1} a_{i} z^{-i}}{1-\sum_{j=1}^{M} b_{j} z^{-j}}, \quad z=e^{i 2 \pi f \Delta t}
$$

is obtained, where

$$
X(z)=\sum_{n=0}^{\infty} x[n] z^{-n} \quad \text { and } \quad Y(z)=\sum_{n=0}^{\infty} y[n] z^{-n}, \quad z \in \mathbb{C} .
$$

By factorization of the transfer function, a representation by poles $d_{j}$ and zeros $c_{i}$ in the complex plane results, which is unique except for a constant factor.

$$
H(z)=\mathrm{const} \cdot \frac{\prod_{i=0}^{N-1}\left(1-c_{i} z^{-1}\right)}{\prod_{j=1}^{M}\left(1-d_{j} z^{-1}\right)}
$$

In order to obtain a causal and stable filter, in the sense that a bounded input generates a bounded output, all poles $d_{j}$ have to be within the unit-circle. The impulse response, i.e. the output $y[n]$ of equation (4.2) for $x[n]=[1,0,0, \ldots, 0]$, is a second way to describe the filter. If all coefficients $b_{j}$ equal zero the impulse response will be determined by the transversal coefficients $a_{i}$ and is finite in time, which names this class of filters as Finite Impulse Response filters (FIR). In all other cases, i.e. if the filter is recursive, the impulse response will have infinite length, naming IIR-filters. The evaluation of the Z-Transform $H(z)$ yields the frequency response function, equivalent to the Fourier transform of the impulse response. In general each transfer function can be approximated by IIR-filters or FIR-filter, but the number of coefficients needed by FIR-filter approximation can be enormously higher. This disadvantage is often accepted in the application of adaptive filters to avoid the risk of instability by moving poles outside the unit-circle. Therefore only FIR-filters are applied in the present investigation.

\subsubsection{Least-mean-square (LMS) algorithm}

Figure 4.1 shows a block diagram of an adaptive filter consisting of an FIR-filter and an algorithm for the adaptation of its coefficients. Generally, the algorithm adapts the coefficients by minimizing a chosen cost-function.

By noting the filter-weights in vector-notation

$$
\mathbf{w}=\left(w_{0}, w_{1}, \ldots, w_{N-1}\right)^{\mathbf{T}},
$$




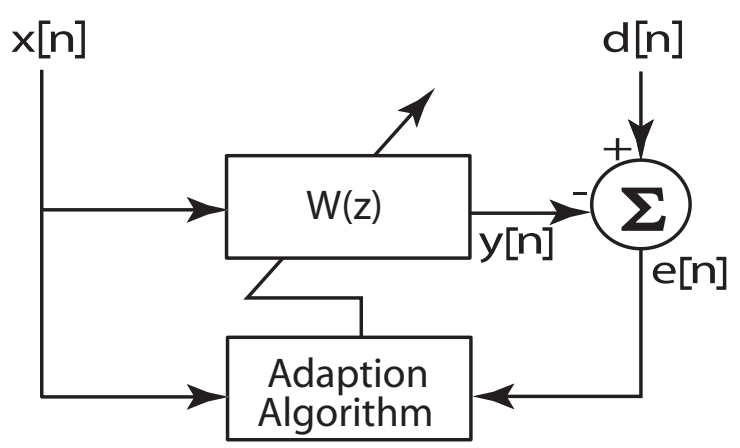

Figure 4.1: The principal scheme of an adaptive filter.

the output signal $y[n]$, obtained by the discrete convolution of the input signal $x[n]$ and the filter vector, can be expressed as the inner product:

$$
\begin{aligned}
y[n] & =\sum_{i=0}^{N-1} w_{i} x[n-i] \\
& =\mathbf{w}^{T} \mathbf{x}[n]=\mathbf{x}^{T}[n] \mathbf{w} .
\end{aligned}
$$

Hence, the error signal $e[n]$ can be written as the difference between the output signal $y[n]$ and the desired signal $\mathrm{d}[\mathrm{n}]$,

$$
\begin{aligned}
e[n] & =d[n]-y[n] \\
& =d[n]-\mathbf{x}^{T}[n] \mathbf{w} .
\end{aligned}
$$

There are different possible choices of the cost function, which is to be minimized, to find the optimum weight vector $\mathbf{w}^{\mathbf{o}}$. A frequently used reasonable choice is to minimize the expected mean squared error.

$$
\begin{aligned}
J[n] & =\left\langle e^{2}[n]\right\rangle \\
& =\left\langle d[n]^{2}\right\rangle-2 \mathbf{w}^{T}\langle d[n] \mathbf{x}[n]\rangle+\mathbf{w}^{T}\left\langle\mathbf{x}[n] \mathbf{x}^{T}[n]\right\rangle \mathbf{w}
\end{aligned}
$$

$\left\langle d[n]^{2}\right\rangle-2 \mathbf{p}^{T} \mathbf{w}+\mathbf{w}^{T} \mathbf{R} \mathbf{w}$ with the crosscorrelation vector

$$
\mathbf{p}=\langle d[n] \mathbf{x}[n]\rangle,
$$

and the autocorrelation matrix

$$
\mathbf{R}=\left\langle\mathbf{x}[n] \mathbf{x}^{T}[n]\right\rangle
$$


is a positive definite quadratic form of the filter weights. Searching the well-defined global minimum

$$
\nabla J[n]=2 \mathbf{R w}-2 \mathbf{p} \stackrel{!}{=} 0,
$$

obtains the discrete form of the Wiener-Hopf equation

$$
\mathbf{R} \mathbf{w}^{\mathbf{0}}=\mathbf{p}
$$

The autocorrelation matrix $\mathbf{R}$ of a stationary process is Toeplitz and is therefore positive semi-definite. In particular for a spectrally rich signal $x[n], \mathbf{R}$ becomes positive definite and thus is guaranteed to be invertible, and the optimum filter, the Wienerfilter, is obtained from equation (4.16):

$$
\mathbf{w}^{\mathbf{0}}=\mathbf{R}^{-1} \mathbf{p} .
$$

By applying the Fourier transform to equation (4.17), it becomes obvious that the spectral density of the input signal $S_{x, x}(f)$ must not contain any zeros,

$$
W^{0}(f)=\frac{S_{x, d}(f)}{S_{x, x}(f)}
$$

Even though there are efficient algorithms for the inversion of Toeplitz matrices, for real-time control applications the computational burden may still be too high, especially for non-stationary processes to be modelled. This means, depending on the degree of non-stationarity, it might be necessary to invert $\mathbf{R}$ even in every time-step.

Due to the quadratic form, i.e. due to the well-defined minimum of the cost-function $J[n]$, a steepest-descent gradient method can be used to find the minimum iteratively. This leads to the following update-algorithm for the filter weights:

$$
\begin{aligned}
\mathbf{w}[n+1] & =\mathbf{w}[n]-\frac{\mu}{2} \nabla J[n] \\
& =\mathbf{w}[n]-\mu \mathbf{R} \mathbf{w}[n]+\mu \mathbf{p} .
\end{aligned}
$$

Even though the inversion of the autocorrelation matrix needs not to be calculated explicitly, $\mathbf{R}$ and $\mathbf{p}$ have to be estimated. The expectation value of the squared error $\left\langle e^{2}[n]\right\rangle$ is replaced by the actual squared error $e^{2}[n]$ in the cost-function, yielding

$$
\nabla J[n]=-2 \mathbf{x}[n] e[n] .
$$

This simplification leads to a stochastic gradient approach of the optimum weight vector, well-known as the least-mean-square algorithm LMS as introduced by Widrow (1970):

$$
\mathbf{w}[n+1]=\mathbf{w}[n]+\mu \mathbf{x}[n] e[n] .
$$


A closer look to this formula shows that the weights are updated by an estimated crosscorrelation between the last $N$ time-shifted samples of the input signal $\mathbf{x}[n]$ and the error signal $e[n]$. Therefore the output signal $y[n]$ of the filter contains such components of the error signal which are correlated with the input signal. To obtain the error $e[n]$, these components will be subtracted from the original signal $d$. This procedure will therefore successively decorrelate the input signal and the error signal, leading to a decrease of latter. Thus the filter is adapting as long as a correlation between both signals exists.

The choice of the convergence parameter $\mu$ is crucial to the performance. Particularly, the speed of convergence, the stability, and the remaining error are determined by the choice of $\mu$. In order to guaranty the convergence of the LMS-algorithm, $\mu$ has to be chosen below a certain limit which depends on the power of the input signal vector $P_{x}=\left\langle x^{2}[n]\right\rangle$ :

$$
0<\mu<\frac{2}{N P_{x}}
$$

The highest possible speed of convergence depends on the statistics of the input signal, the eigenvalue spread of $\mathbf{R}$ governs the highest possible speed of convergence. The eigenvalue spread can be estimated from the ratio of the highest and the smallest component in the spectrum of the input signal $|X(f)|^{2}$ :

$$
1 \leq \frac{\lambda_{\max }}{\lambda_{\min }} \leq \frac{\max |X(f)|^{2}}{\min |X(f)|^{2}}
$$

However, in practice, no simple way of finding the optimum $\mu$ is known. For this reason a lot of research in pre-whitening technics has been carried out in the last decade. Nevertheless the LMS-algorithm is very popular for real-time applications because of its low computational cost of $O(2 \mathrm{~N})$ and its robustness. Therefore it is used in the present study for the wave cancellation experiments, whereas for the system identification the stabilized fast transversal filter algorithm (sFTF) (Slock and Kailath 1991) has been used because of its significant time savings. This algorithm is a modified recursive least squares algorithm (RLS), which inverts $\mathbf{R}$ recursively and therefore is independent on the input signal statistics. The sFTF algorithm reduces the numerical cost $O\left(N^{2}\right)$ of the RLS-algorithm by forward and backward prediction methods to $O(8 N)$. Depending on the complexity of the problem, the sFTF-algorithm is also used in real-time control applications, e.g. Opfer and Guicking (1999). Detailed derivations and descriptions of the specific properties of the mentioned algorithms can be found in the literature, e.g. Haykin (1996), Alexander (1986), and Kuo and Morgan (1996). 


\subsection{Active wave cancellation (AWC)}

This section describes the application of adaptive filters with regard to the requirements of the present study. Starting with the procedure of system identification, the configuration of a single-input-single output (SISO) system is extended to a multipleinput-multiple-output (MIMO) set-up.

\subsubsection{System identification}

The linear system identification is a task an adaptive filter is obviously able to handle. The general principle is that the system is excited by means of a disturbance generator or an actuator, which is supplied by a random white noise signal $x[n]$ and the excitation is measured at the output of the system, e.g. a sensor. The obtained signal serves as the desired signal $d[n]$ and $x[n]$ as input signal of the adaptation algorithm. Fig. 4.2 shows the system identification scheme in order to estimate the secondary path $\hat{S}(z)$. The adaptive filter models hereby the physical transfer function $P(z)=S^{\prime}(z)$ of the system including the transfer functions of the generation process $H_{a}(z)$ and of the measuring process $H_{e}(z)$,

$$
W(z)=H_{a}(z) P(z) H_{e}(z) .
$$

In the system investigated here the transfer functions $H_{a}(z)$ and $H_{e}(z)$ will imply a number of processes. $H_{a}(z)$ contains the DA-conversion, the reconstruction lowpass filters, the amplifiers, the actuator, and the receptivity of the boundary layer with respect to the excited wall-normal fluid fluctuations. $H_{e}(z)$ implies the sensitivity of the sensor, the CTA, the amplifiers, the anti-aliasing lowpass filters, and the AD-conversion. By a modification, the system identification scheme can also be used to estimate the physical transfer functions alone. For this purpose the influence of the transfer functions linked to the processes of generation and measurement is considered first, namely by minimizing the distance between the sensor and the disturbance generator and estimating these transfer functions by means of a first system identification. In fact, the sensor is placed directly above the disturbance generator yielding the adapted filter $W_{0}(z) \approx \hat{H}_{a}(z) \hat{H}_{e}(z)$. Once this estimate is obtained, it can be taken into account in the system identification to obtain an estimate of the physical transfer path, exclusively. The input signal $x[n]$ is pre-filtered by the estimate $W_{0}(z)$ for this purpose, and the adaptive filter

$$
W(z)=H_{a}(z) P(z) H_{e}(z) / W_{0}(z) \approx P(z)
$$

is obtained. Depending on the accuracy of the estimates $\hat{H}_{a}(z)$ and $\hat{H}_{e}(z)$, the optimum weights of the adaptive filter $\mathbf{w}^{\mathbf{o}}$ represent an approximation of the physical transfer function $P(z)$. In particular in the case of an FIR-filter, regarding the example shown 


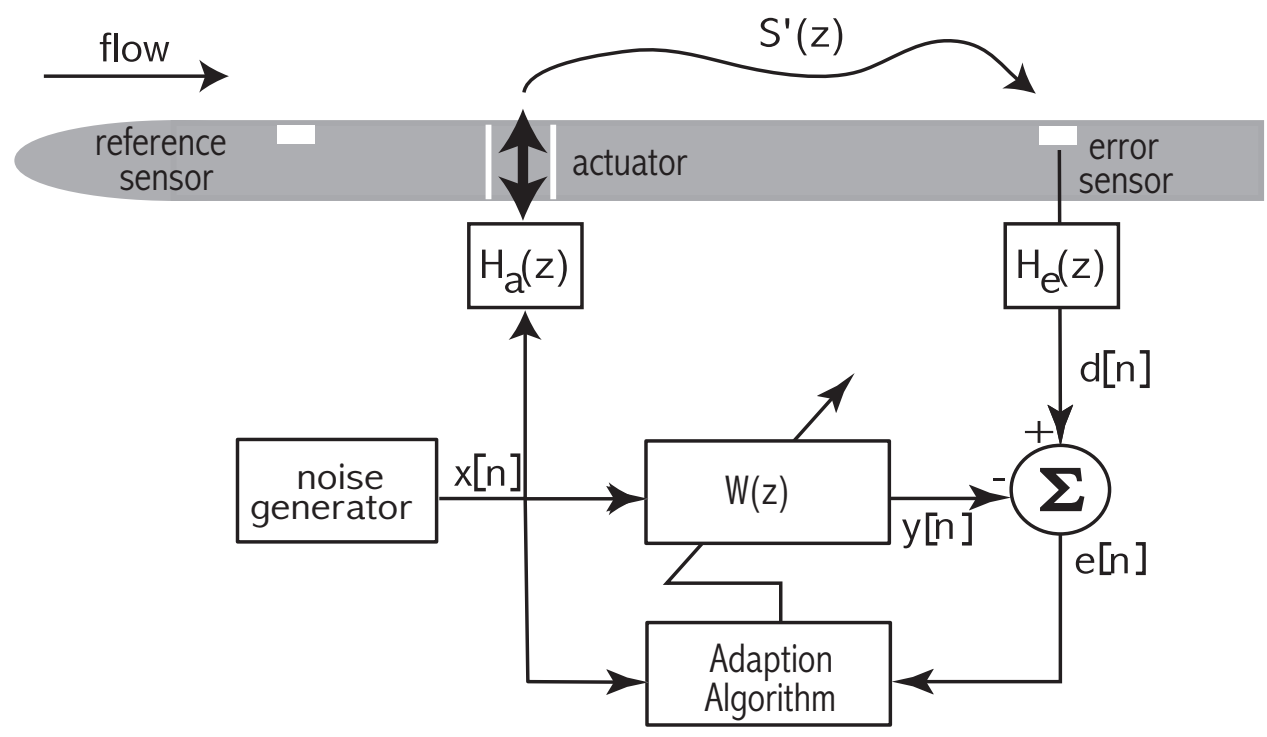

Figure 4.2: The system identification scheme for estimation of the secondary path.

in Fig. 4.2, the weights immediately represent the impulse response of the boundary layer between the actuator and the error sensor.

\subsubsection{Single-input-single-output (SISO) system}

To explain the principles of the active wave cancellation methods, it is reasonable to start with a single-input-single-output system which means a system with a single reference sensor, an actuator, and a single error sensor, as shown in Fig. 4.3. The boundary layer disturbance of whatever origin is detected by the reference sensor. While the disturbance propagates downstream to the actuator, the so-called primary path $A(z)$, the measured signal $x[n]$ is the input to the adaptive filter that generates an output signal $y[n]$ driving the actuator to produce a second artificial disturbance. In order to cancel the primary disturbance by superposition of a counter-phase wave, the adaptive filter has to be adjusted according to

$$
W(z)=\frac{A(z)}{H_{r}(z) H_{a}(z)}
$$

and the sign of the output signal $y[n]$ has to be inverted. 




Figure 4.3: The active wave cancellation scheme for a single reference sensor, actuator, and error sensor.

In order to decorrelate the reference signal and error signal, the reference signal has to be aligned to the error signal such that the delay of the filter-output signal, caused by the transfer functions $H_{a}(z), S^{\prime}(z)$, and $H_{e}(z)$, is taken into account. Initially, the overall transfer function is estimated via the described system identification scheme yielding the estimate $\hat{S}(z)$ of the secondary path $S(z)=H_{a}(z) S^{\prime}(z) H_{e}(z)$. This estimate is considered in a further modification of the adaptation algorithm, namely due to pre-filtering of the input signal with $\hat{S}(z)$ (see Fig. 4.4). The resulting filterupdate formula

$$
\mathbf{w}[n+1]=\mathbf{w}[n]+\mu\{\hat{\mathbf{s}} * \mathbf{x}\}[\mathbf{n}] \mathbf{e}[\mathbf{n}]
$$

is known as the filtered-X-LMS and was independently derived by Widrow, Shur, and Shaffer (1981) and Burgess (1981). Herein the sign $*$ denotes the discrete convolution between the reference signal and the filter vector of the secondary path estimate

$$
\hat{\mathbf{s}}=\left(\hat{s}_{0}, \hat{s}_{1}, \ldots, \hat{s}_{N-1}\right)^{\mathbf{T}} \text {. }
$$

The derivation is based on the theory of linear time invariant (LTI) systems, in particular on the property that the sequence of subsystems can be changed $(\{\hat{s} * x\}=\{x * \hat{s}\})$, which is described in an intuitive way by Evert (2000). The filtered-X-LMS algorithm 
will precisely compensate for the phase shift introduced by the secondary path. If $\hat{S}(z) \neq S(z)$, the secondary-path estimate has to be at least accurate enough to match the phase within 90 degrees for the algorithm to be stable; however, the accuracy of the estimate does effect the performance of the adaptive controller in terms of convergence speed (Snyder and Hansen 1994).

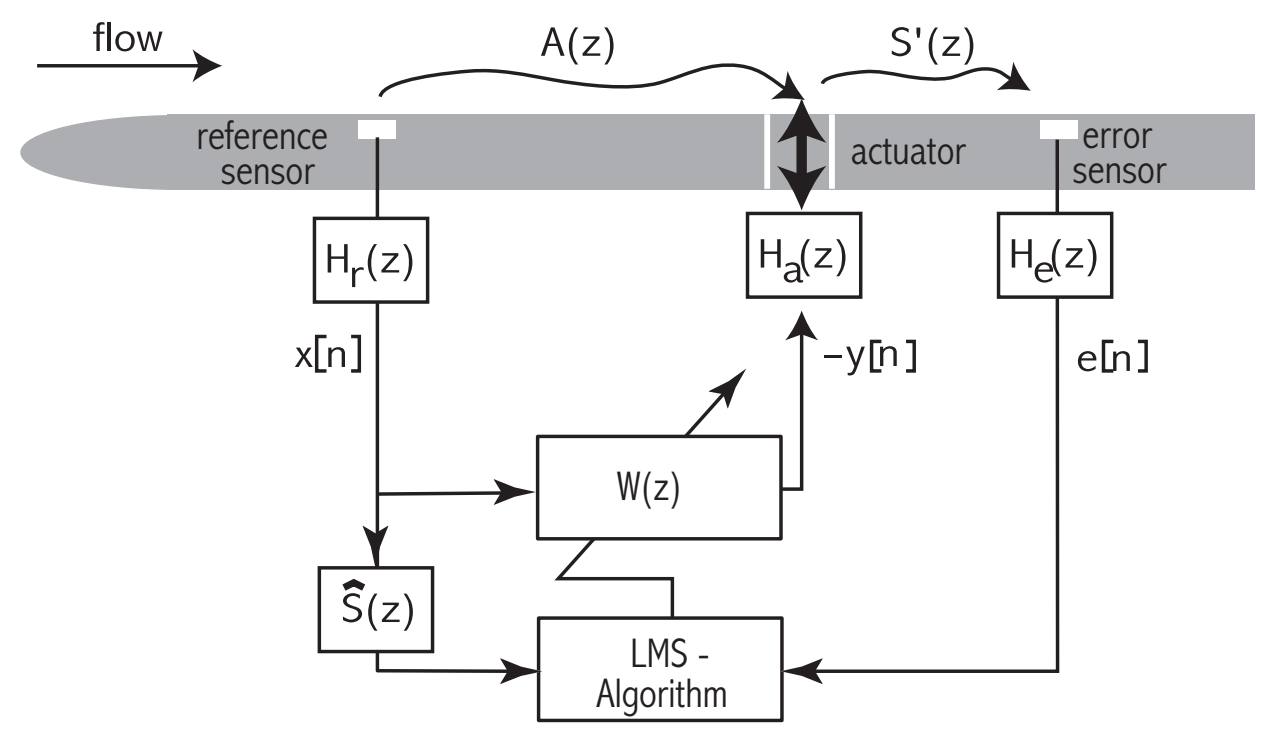

Figure 4.4: The block diagram of the filtered-X-LMS algorithm for active wave cancellation.

In praxis, uncorrelated noise will be present in the measured signals. Therefore the remaining power of the error sensor signal after adaptation, and herewith the performance of the system, can be estimated beforehand by the coherence function

$$
C_{x d}(f)=\left|\gamma_{x d}(f)\right|^{2}=\frac{\left|S_{x d}(f)\right|^{2}}{S_{d d}(f) S_{x x}(f)}
$$

measured between the reference signal $x$ and the desired signal $d$. The spectrum (PSD) of the remaining error signal is given by

$$
S_{e e}(f)=\left[1-C_{x d}(f)\right] S_{d d}(f),
$$

where $C_{x d}(f) S_{d d}(f)$ describes the part of the spectral density correlated to the reference signal. The relation between this part and the original desired signal spectrum 
determines the maximum possible reduction of the error sensor spectrum

$$
-10 \log _{10}\left[1-C_{x d}(f)\right]
$$

\subsubsection{Multiple-input-multiple-output (MIMO) system}

A system of $J$ reference sensors, $K$ actuators, and $M$ error sensors will be considered. In order to find the appropriate algorithm that eliminates all correlations between the reference and the error signals, one may start by comprising the measured signal vectors into generalized vectors to simplify the notation.

The error signal vector ${ }^{1}$

$$
\mathbf{e}[n] \equiv\left(e_{1}[n], e_{2}[n], \ldots, e_{M}[n]\right)^{T}
$$

measured by the $M$ error sensors can be expressed as the difference between $M$ desired output signals summarized in the vector

$$
\mathbf{d}[n] \equiv\left(d_{1}[n], d_{2}[n], \ldots, d_{M}[n]\right)^{T}
$$

and the cancelling signal vector $\mathbf{y}^{\prime}[n]$ :

$$
\mathbf{e}[n]=\mathbf{d}[n]-\mathbf{y}^{\prime}[n] .
$$

$\mathbf{y}^{\prime}[n]$ can be calculated at each of the $M$ error sensors by the summation of all $K$ components generated by the actuators. Equivalently, the actuator signal vector

$$
\mathbf{y}[n] \equiv\left(y_{1}[n], y_{2}[n], \ldots, y_{K}[n]\right)^{T}
$$

being the input of the $K$ actuators can be filtered by the $M \times K$ matrix of the secondary path transfer functions

$$
\mathbf{S}=\left[\begin{array}{cccc}
\mathbf{s}_{11}[n] & \mathbf{s}_{12}[n] & \cdots & \mathbf{s}_{1 K}[n] \\
\mathbf{s}_{21}[n] & \mathbf{s}_{22}[n] & \cdots & \mathbf{s}_{2 K}[n] \\
\vdots & \vdots & \ddots & \vdots \\
\mathbf{s}_{M 1}[n] & \mathbf{s}_{M 2}[n] & \cdots & \mathbf{s}_{M K}[n]
\end{array}\right]
$$

containing all $M \times K$ impulse responses $\mathbf{s}_{m k}[n]$ (denoting the response of the $m$ 'th error sensor to the $k^{\prime}$ th actuator). Thus, $y^{\prime}[n]$ can be written for each element separately as

\footnotetext{
${ }^{1}$ Note that the signal vectors are here (in the MIMO-case) defined differently, namely comprising the current samples of each array-component (sensor,actuator) and the index denotes the component.
} 
the summation over the outputs of the convolution of the each signal $y_{k}[n]$, driving the $k$ 'th actuator, with the corresponding secondary transfer function to $m$ 'th error sensor:

$$
\mathbf{y}_{m}^{\prime}[n]=\sum_{k=1}^{K} \mathbf{s}_{m k}[n] * y_{k}[n], \quad m=1,2, \ldots, M .
$$

This can be written in vector notation as

$$
\mathbf{y}^{\prime}[n] \equiv \mathbf{S}[n] * \mathbf{y}[n]
$$

$\mathbf{y}[n]$ can be obtained in a similar way by the convolution, and the subsequent summation, of the reference signals with the corresponding adaptive weight vectors driving a specified actuator.

To start with, $\mathbf{y}[n]$ can be written in vector form as

$$
\mathbf{y}[n]=\mathbf{X}^{T}[n] \mathbf{w}[n] .
$$

where

$$
\mathbf{X}[n] \equiv\left[\begin{array}{cccc}
\mathbf{x}[n] & 0 & \cdots & 0 \\
0 & \mathbf{x}[n] & \ddots & \vdots \\
\vdots & \ddots & \ddots & 0 \\
0 & \cdots & 0 & \mathbf{x}[n]
\end{array}\right]
$$

is a $J K N \times K$ matrix containing the $J N \times 1$ generalized reference signal vectors $\mathbf{x}[n]$, and $\mathbf{w}$ is the $J K N \times 1$ generalized adaptive weight vector. The generalized reference vector

$$
\mathbf{x}[n] \equiv\left(\mathbf{x}_{1}^{T}[n], \mathbf{x}_{2}^{T}[n], \ldots, \mathbf{x}_{J}^{T}[n]\right)^{T}
$$

comprises the $J$ signal vectors of each of the reference sensors

$$
\mathbf{x}_{j}[n] \equiv\left(x_{j}[n], x_{j}[n-1], \ldots, x_{j}[n-N+1]\right)^{T}, \quad j=1,2, \ldots, J
$$

that are arranged one after the other. The $J K N \times 1$ generalized adaptive weight vector $\mathbf{w}$

$$
\mathbf{w} \equiv\left[\mathbf{w}_{1}^{T}, \mathbf{w}_{2}^{T}, \ldots, \mathbf{w}_{K}^{T}\right]^{T},
$$

is formed by the $K$ subvectors

$$
\mathbf{w}_{k} \equiv\left(\mathbf{w}_{(k, 1)}^{T}, \mathbf{w}_{(k, 2)}^{T}, \ldots, \mathbf{w}_{(k, J)}^{T}\right)^{T}, \quad k=1,2, \ldots, K .
$$


Each of the $K$ subvectors contains all $J$ weight vectors driving a specified actuator , each of the form

$$
\mathbf{w}_{(k, j)} \equiv\left(w_{0}, w_{1}, \ldots, w_{N-1}\right)^{T}
$$

Using these notations equation (4.35) can be written as

$$
\begin{aligned}
\mathbf{e}[n] & =\mathbf{d}[n]-\mathbf{S}[n] * \mathbf{y}[n] \\
& =\mathbf{d}[n]-\mathbf{S}[n] *\left\{\mathbf{X}^{\mathbf{T}}[n] \mathbf{w}\right\} .
\end{aligned}
$$

Assuming the weight vector changes slowly, the theory of linear time invariant systems may be applied and thus the term $\mathbf{S}[n] *\left\{\mathbf{X}^{\mathbf{T}}[n] \mathbf{w}\right\}$ may be rewritten as $\mathbf{X}^{\prime \mathbf{T}}[n] \mathbf{w}$, where

$$
\begin{aligned}
\mathbf{X}^{\prime}[n] & \equiv \mathbf{S}^{\mathbf{T}}[n] * \mathbf{x}[n] \\
& =\left[\begin{array}{cccc}
\mathbf{s}_{11}[n] * \mathbf{x}[n] & \mathbf{s}_{12}[n] * \mathbf{x}[n] & \cdots & \mathbf{s}_{1 K}[n] * \mathbf{x}[n] \\
\mathbf{s}_{21}[n] * \mathbf{x}[n] & \mathbf{s}_{22}[n] * \mathbf{x}[n] & \cdots & \mathbf{s}_{2 K}[n] * \mathbf{x}[n] \\
\vdots & \vdots & \ddots & \vdots \\
\mathbf{s}_{M 1}[n] * \mathbf{x}[n] & \mathbf{s}_{M 2}[n] * \mathbf{x}[n] & \cdots & \mathbf{s}_{M K}[n] * \mathbf{x}[n]
\end{array}\right] .
\end{aligned}
$$

denotes the $J K N \times M$ matrix of the filtered reference signals.

As in the single-input-single-output case, there are different possible choices of the cost function $J[n]$, that is to be minimized, in order to find the optimum set of weight vectors $\mathbf{w}^{o}$. A reasonable choice is minimizing the sum of the expected mean squared errors, measured at the $M$ error sensors.

$$
\begin{aligned}
J[n] & =\sum_{m=1}^{M}\left\langle e^{2}[n]\right\rangle=\left\langle\mathbf{e}^{\mathbf{T}}[n] \mathbf{e}[n]\right\rangle \\
& =\left\langle\mathbf{d}^{\mathbf{T}}[n] \mathbf{d}[n]\right\rangle-2 \mathbf{w}^{T} \underbrace{\left\langle\mathbf{X}^{\prime \mathbf{T}}[n] \mathbf{d}[n]\right\rangle}_{\equiv \mathbf{p}_{\mathbf{m}}}+\mathbf{w}^{T} \underbrace{\left\langle\mathbf{X}^{\prime \mathbf{T}}[n] \mathbf{X}^{\prime}[n]\right\rangle}_{\equiv \mathbf{R}_{\mathbf{m}}} \mathbf{w} \\
& =\left\langle\mathbf{d}^{\mathbf{T}}[n] \mathbf{d}[n]\right\rangle-2 \mathbf{w}^{T} \mathbf{p}_{\mathbf{m}}+\mathbf{w}^{T} \mathbf{R}_{\mathbf{m}} \mathbf{w}
\end{aligned}
$$

$\mathbf{p}_{\mathbf{m}}$ contains all cross-correlations between the $J$ reference signals filtered by the secondary transfer functions and the $M$ error sensors. $\mathbf{R}_{\mathbf{m}}$ denotes the autocorrelation of the filtered reference signals. The quadratic nature of the error surface can now be clearly identified, and it can be confirmed that the surface has a unique global minimum by examining the positive definiteness of $\mathbf{R}_{\mathbf{m}}$. By setting the differential of this expression with respect to $\mathbf{w}$ to zero, the optimum Wiener set of coefficients may be obtained (Elliott, Stothers, and Nelson 1987):

$$
\mathbf{w}^{\mathbf{0}}=\mathbf{R}_{\mathbf{m}}{ }^{-1} \mathbf{p}_{\mathbf{m}} .
$$


Knowing that the cost function $J[n]$ has a unique minimum one can apply a steepestdescent gradient method to find the minimum by iteration. This leads to the updatealgorithm for the generalized filter weights:

$$
\mathbf{w}[n+1]=\mathbf{w}[n]-\frac{1}{2} \mu \nabla J[n]
$$

Assuming that each mean square error component is approximated by its instantaneous squared error the gradient is calculated with respect to the $k^{\prime}$ th weight subvector $\mathbf{w}_{\mathbf{k}}[n]$ at time $n$, using the equations (4.48) and (4.47):

$$
\begin{aligned}
\nabla_{k} J[n] & =\nabla_{k} \sum_{m=1}^{M} e^{2}[n] \\
& =-2 \sum_{m=1}^{M}\left\{\mathbf{s}_{m k}[n] * \mathbf{x}[n]\right\} e_{m}[n] \quad k=1,2, \ldots, K
\end{aligned}
$$

Combining this equation and the equation (4.51) leads to the multiple-inputmultiple-output filtered-X algorithm as introduced by Elliott, Stothers, and Nelson (1987):

$$
\mathbf{w}[n+1]=\mathbf{w}[n]+\mu \mathbf{X}^{\prime}[n] \mathbf{e}[n]
$$

The components can be expressed as

$$
\begin{array}{r}
\mathbf{w}_{k j}[n+1]=\mathbf{w}_{k j}[n]+\mu \sum_{m=1}^{M}\left\{\mathbf{s}_{m k}[n] * \mathbf{x}_{j}[n]\right\} e_{m}[n] \\
k=1,2, \ldots, K \quad j=1,2, \ldots, J .
\end{array}
$$

In real applications the matrix $\mathbf{S}$ is usually not available, therefore it is substituted by an estimate $\hat{\mathbf{S}}$. To illustrate the obtained algorithm, Fig. 4.5 shows a block diagram of a two-input-two-output system. For this case the update formula (4.54) can be written as:

$$
\begin{aligned}
& \mathbf{w}_{\mathbf{1 1}}[n+1]=\mathbf{w}_{11}[n]-\mu\left[\left\{\mathbf{s}_{11}[n] * \mathbf{x}_{\mathbf{1}}[n]\right\} e_{1}[n]+\left\{\mathbf{s}_{21}[n] * \mathbf{x}_{\mathbf{1}}[n]\right\} e_{2}[n]\right] \\
& \mathbf{w}_{\mathbf{2 1}}[n+1]=\mathbf{w}_{21}[n]-\mu\left[\left\{\mathbf{s}_{12}[n] * \mathbf{x}_{\mathbf{1}}[n]\right\} e_{1}[n]+\left\{\mathbf{s}_{22}[n] * \mathbf{x}_{\mathbf{1}}[n]\right\} e_{2}[n]\right] \\
& \mathbf{w}_{\mathbf{1 2}}[n+1]=\mathbf{w}_{12}[n]-\mu\left[\left\{\mathbf{s}_{11}[n] * \mathbf{x}_{\mathbf{2}}[n]\right\} e_{1}[n]+\left\{\mathbf{s}_{21}[n] * \mathbf{x}_{\mathbf{2}}[n]\right\} e_{2}[n]\right] \\
& \mathbf{w}_{\mathbf{2 2}}[n+1]=\mathbf{w}_{22}[n]-\mu\left[\left\{\mathbf{s}_{12}[n] * \mathbf{x}_{\mathbf{2}}[n]\right\} e_{1}[n]+\left\{\mathbf{s}_{22}[n] * \mathbf{x}_{\mathbf{2}}[n]\right\} e_{2}[n]\right]
\end{aligned}
$$






Figure 4.5: Scheme of the MIMO active wave cancellation system consisting of 2 reference sensors, 2 actuators (sharing one slit), and 2 error sensors. The 4 adaptive filters (marked in green) are optimized by the 2-input/2-output filtered-X-LMS algorithm.

\subsubsection{Convergence and stability of the MIMO-filtered-X-LMS}

The behavior of the MIMO filtered-X-LMS for an arbitrary wideband signal, as derived above, is difficult to analyze. The optimum steady state Wiener filter given in equation (4.50) shows that the condition of the autocorrelation matrix of the filtered input signal, which contains temporal and spatial characteristics, will play a key role.

However, for narrowband signals it is possible to analyze some aspects of the algorithm convergence (Elliott, Boucher, and Nelson 1992). The spatial distribution of the actuators and the error sensors will have two principal consequences that effect the convergence of the algorithm: the eigenvalue structure of the relevant matrices and the delay caused by their physical separation.

Following the analysis of a single frequency component, which can be considered a constant value $X=1$ in the frequency domain, the error signal vector can be expressed in the frequency domain by ${ }^{2}$

$$
\mathbf{E}=\left[E_{1}, E_{2}, \ldots, E_{M}\right]^{T}
$$

\footnotetext{
${ }^{2}$ Note the deviation from the convention of notation so far using bold letters for matrices.
} 


$$
=\mathbf{D}-\mathrm{SW},
$$

where $\mathbf{D}$ and $\mathbf{W}$ denote the vectors comprising desired signal and adaptive weights in the frequency-domain. By choosing the well-known cost-function

$$
J[n]=\sum_{m=1}^{M} E_{m}^{2}=\mathbf{E}^{\mathbf{H}} \mathbf{E}
$$

and taking the complex gradient $\nabla_{\mathbf{W}^{\mathbf{H}}} J$, the MIMO frequency-domain-filtered-X-LMS algorithm is obtained:

$$
\mathbf{W}[\mathbf{n}+\mathbf{1}]=\mathbf{W}[\mathbf{n}]+\mu \mathbf{S}^{\mathbf{H}} \mathbf{E}[\mathbf{n}] .
$$

The steady state optimum weight solution in the Wiener sense is given by

$$
\mathbf{W}_{\text {opt }}=\left(\mathbf{S}^{\mathbf{H}} \mathbf{S}\right)^{-1} \mathbf{S}^{\mathbf{H}} \mathbf{D} \text {. }
$$

By comparison to the mean weight convergence of the SISO time-domain LMS algorithm, see equation (3.19), we see that the matrix $\mathbf{S}^{\mathbf{H}} \mathbf{S}$ plays the same role as the reference signal autocorrelation matrix in the former case. Thus, the eigenvalues of $\mathbf{S}^{\mathbf{H}} \mathbf{S}$ determine the convergence characteristics of the MIMO frequency-domain filtered-X-LMS algorithm. It is noteworthy that disparate eigenvalues arise here because of the spatial nature of the secondary-path matrix $\mathbf{S}$. This occurs even though the temporal characteristics of the reference signal are well conditioned, in fact it was shown that the effects of the temporal characteristics are usually much smaller than the spatial ones (Elliott, Boucher, and Nelson 1992).

Thus, convergence of the general broadband MIMO filtered-X-LMS would be expected to be limited by both the spatial and temporal characteristics of the system (Kuo and Morgan 1996).

In order to enhance the robustness of the MIMO filtered-X-LMS, the cost-function (equation 4.49) can be modified in a way that the output of the controller is penalized,

$$
J[n]=\left\langle\mathbf{e}^{\mathbf{T}}[n] \mathbf{e}[n]+\beta \mathbf{y}^{\mathbf{T}}[n] \mathbf{y}[n]\right\rangle
$$

which results in the introduction of a leakage-factor $\gamma$, with $0<\gamma<1$, in the filterupdate formula 4.54:

$$
\begin{gathered}
\mathbf{w}_{\mathbf{k j}}[n+1]=\gamma \mathbf{w}_{k j}[n]-2 \mu \sum_{m=1}^{M}\left\{s_{m k}[n] * \mathbf{x}_{\mathbf{j}}[n]\right\} e_{m}[n] \\
k=1,2, \ldots, K \quad j=1,2, \ldots, J .
\end{gathered}
$$

Herein, the leakage-factor is determined by $\gamma=1-\mu \sigma^{2} \beta$, where $\sigma$ denotes the estimated power of filter-taps, see e.g. Nelson (1994). This equation is in the form of the leakyLMS algorithm as introduced by Widrow and Streans (1985), where the factor $\gamma$ ensures 
that the algorithm continuously searches for the least effort solution by slightly reducing the value of all the tap weights at each iteration. The introduction of the leakage factor has not been necessary in the present investigation, but may become so in other systems for the above mentioned reasons.

The effect of secondary-path modelling errors, $\hat{S}(z) \neq S(z)$, on the performance of a MIMO control system was also investigated by Elliott, Boucher, and Nelson (1992) for a single reference signal in the single-frequency case. They found that convergence is assured for step-size parameter $\mu$ if

$$
\left|1-\mu \lambda_{k}\right|<1
$$

for all $k$ where $\lambda_{k}$ is the $k$ th eigenvalue of $\left(\hat{\mathbf{S}}^{\mathbf{H}} \mathbf{S}+\mathbf{1}\right)$. This condition is met if

$$
\mu<\frac{2 \Re\left(\lambda_{k}\right)}{\left|\lambda_{k}\right|^{2}}
$$

for all $k \mathrm{~s}$. 


\section{Chapter 5}

\section{Experimental set-up}

In order to choose a proper experimental environment where the application of active wave cancellation methods to the so-called naturally occurring TS-waves in the boundary layer can be investigated, a number of considerations have turned out to be important. As mentioned above the naturally occurring TS-waves are excited due to the receptivity of the boundary layer to external perturbations. In the case of wind tunnel experiments these perturbations can be considered as disturbances which are generated by acoustical noise and the free-stream turbulence in the wind tunnel as well as vibrations of the wind tunnel model itself. Therefore it is necessary to choose a wind tunnel environment where a certain amount of those perturbations is present. On the other hand, to prevent Bypass-transition, the perturbations must not be too strong. Thus, it is necessary to choose an environment with an intermediate level of perturbations.

Furthermore, it is reasonable to choose a flat plate as a wind tunnel model because of the well known properties of its boundary layer. This choice will additionally reduce the number of parameters influencing the results and therefore it becomes easier to generalize the obtained results. Among others this study aims at the investigation of effects caused by the angle dependence of dispersion, which, in turn, depends significantly on the pressure gradient and even disappears when imposing an adverse pressure gradient on the boundary layer, as described in chapter 2.3. Thus conditions close to Blasius flow have been striven for.

Concerning the design of the active wave cancellation device, it has to be mentioned that some of the requirements to such a system as presented in chapter 3 resulted from the experimental investigations presented below and have therefore not been clear from the beginning. 

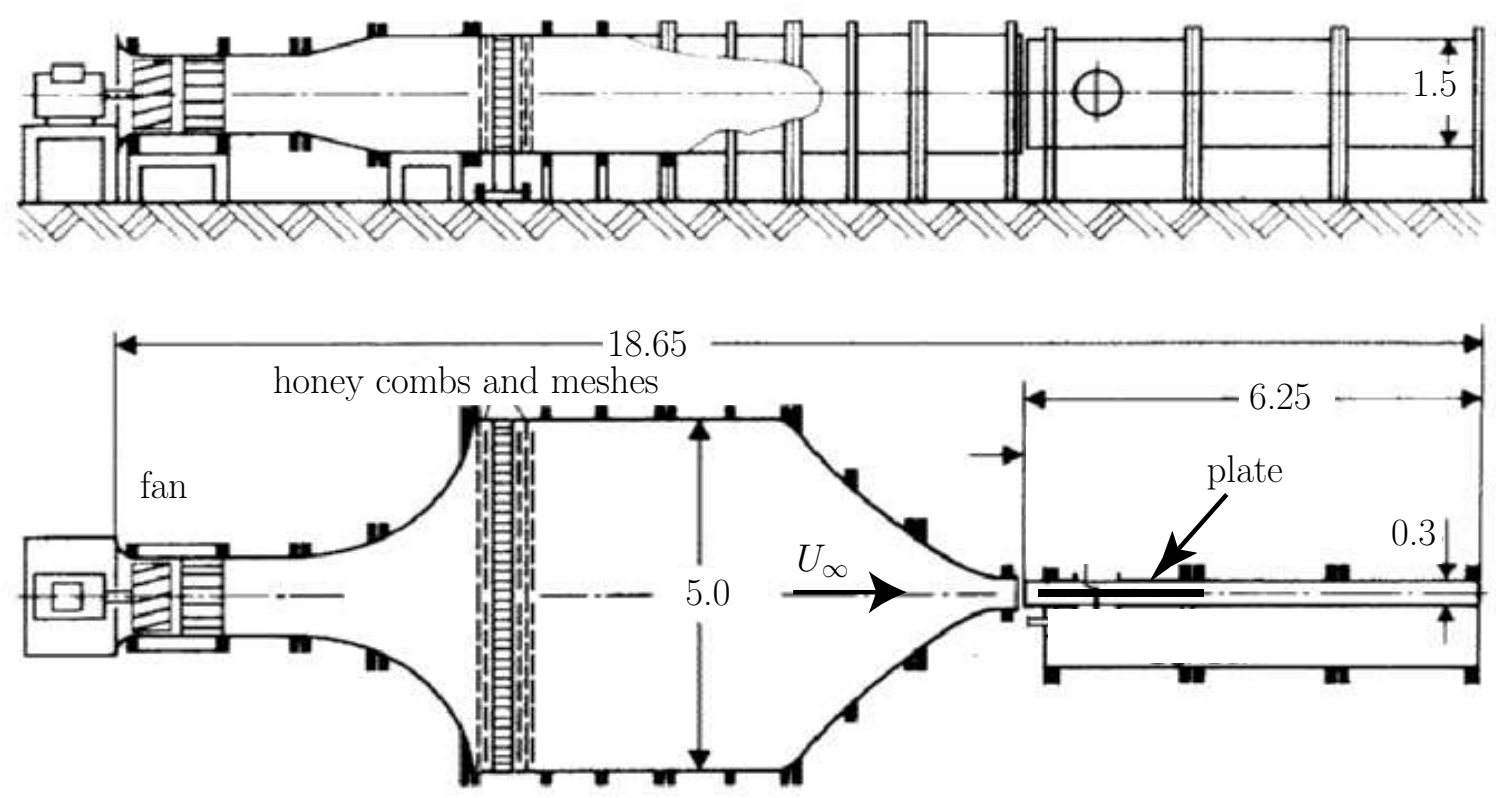

Figure 5.1: The low-turbulence wind tunnel TUG of the DLR Göttingen (side view and top view, all dimensions in meters).

\subsection{The wind tunnel and the experimental equip- ment}

The experiments of the present study were carried out in the low-turbulence wind tunnel TUG of the DLR in Göttingen (Fig. 5.1). The TUG is a continuously operating low speed wind tunnel of Eiffel type with a closed test section and open return through the test hall. The fan is driven by a $80 \mathrm{~kW}$ DC-motor. The velocity in the $1.50 \times 0.3 \mathrm{~m}^{2}$-test section can be varied in the range $4 \mathrm{~m} / \mathrm{s}<U_{\infty}<45 \mathrm{~m} / \mathrm{s}$. Due to the high contraction ratio of 16:1 between the settling chamber and the measurement section and a series of meshes and honey-combs a low turbulence level of $0,065 \%$ of the free stream velocity can be achieved at low speeds in the TUG (Fischer 1994). At higher speeds the TUG becomes quite noisy (about $101 \mathrm{~dB}$ at $U_{\infty}=18 \mathrm{~m} / \mathrm{s}$ ), thus degrading the low-turbulence conditions. 


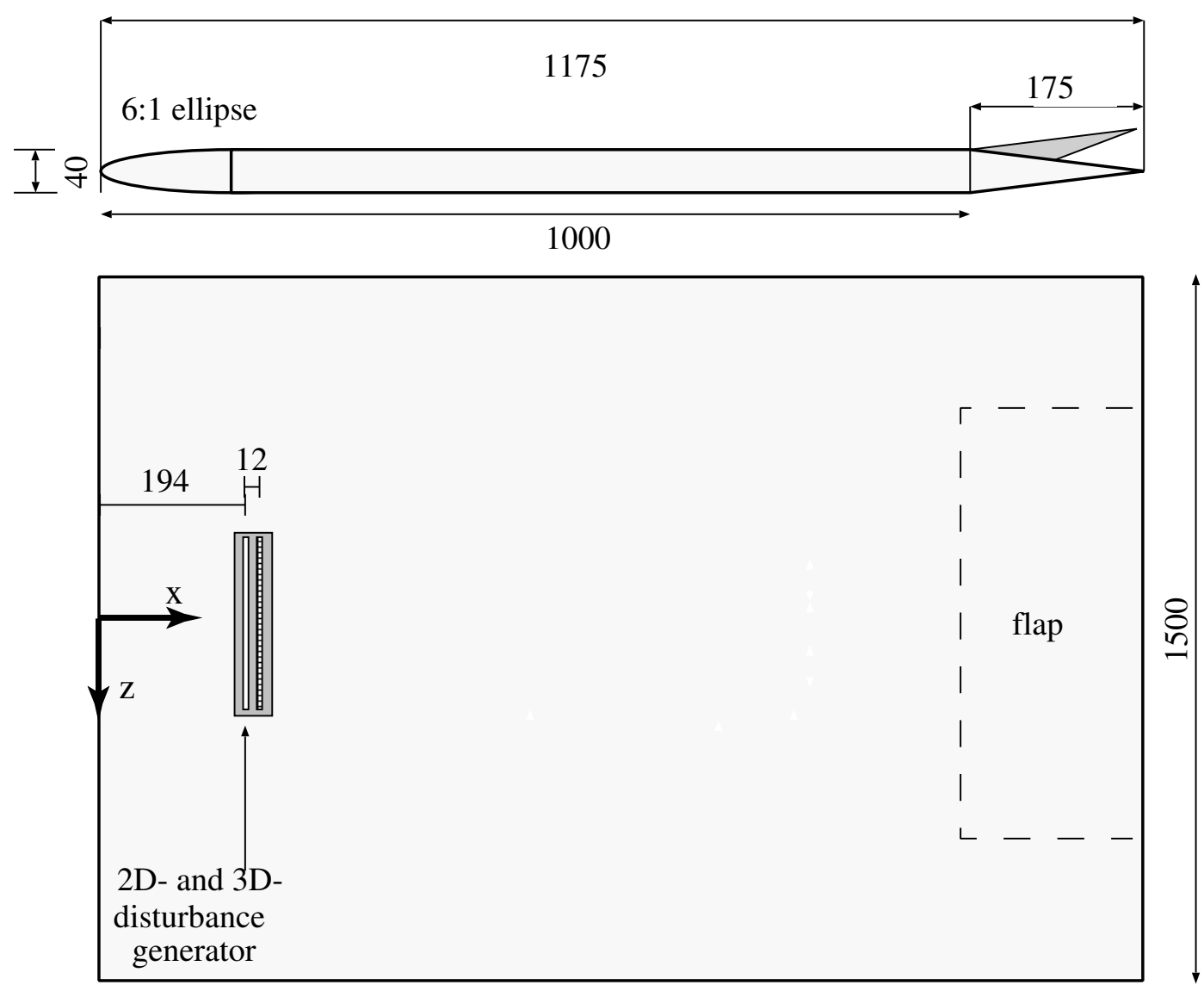

Figure 5.2: Side view and top view of the plate, all dimensions are given in millimeters.

To develop an active wave cancellation device, a flat plate has been chosen as a wind tunnel model, which has been successfully used for a number of investigations in transition research, e.g. (Wiegel 1997; Fischer 1994; Sundaram et al. 1997). The dimensions of the plate are given in Fig. 5.2. The plate was made from plexiglass because of its high surface quality. The averaged degree of roughness is given as $R_{z}=0,2 \mu \mathrm{m}$.

The model is mounted vertically in the test section. External probes can be adjusted close to the surface by introducing the probe holder through a manhole in the wind tunnel wall. This manhole restricts the accessible measurement area in the streamwise coordinate to $100 \mathrm{~mm} \leq x \leq 750 \mathrm{~mm}$ and in the spanwise coordinate to $-210 \mathrm{~mm} \leq z \leq+210 \mathrm{~mm}$, where the origin is chosen to be the center of the leading edge.

In order to prevent boundary layer separation at the leading edge the plate has an elliptic leading edge of ratio $6: 1$, which was made from aluminium. The elliptic 
shape also minimizes the acoustic receptivity of the leading edge (Wlezien, Parekh, and Island 1990). This was seen as an advantage in previous experiments carried out with this plate, because these investigations focussed on artificially introduced disturbances considering naturally occurring TS-waves as irritating side effects. The stagnation point at the leading edge can be shifted by a flap built into the trailing edge. Also the streamwise pressure gradient, which can be used for fine tuning purposes, is influenced by the flap setting.

The plate was already equipped with a $2 \mathrm{D}$-disturbance generator. It consists of a pressure chamber loudspeaker connected via plastic tubes to a chamber in the plate that is connected to the surface of the plate by a $41 \mathrm{~cm}$ long spanwise slit of width $0.3 \mathrm{~mm}$. The acoustic pressure generated in the chamber leads to wall-normal velocity fluctuations in the slit, and the boundary layer above that are known to be effective generators of Tollmien-Schlichting waves.

Additionally, a 3D disturbance generator has been built into the plate just $9 \mathrm{~mm}$ further downstream. It is constructed in a similar way but consists of 40 spanwise slits (10 $\mathrm{mm}$ long and separated by $0.5 \mathrm{~mm}$ wide bridges) each connected to a separate chamber which can also be driven independently by a loudspeaker. Wiegel (1997) used this device to generate oblique TS-waves by varying the phase between the driving signals.

\section{$5.2 \quad$ Hot-wire measurements}

Hot-wire anemometry is often applied to the measurement of velocity fluctuations in the sub- and supersonic range ${ }^{1}$. The method is based on the measurement of the heatloss per time $\dot{Q}$ of an overheated thin metal-wire exposed to the flow. The heat loss is related to the mass flow of the surrounding fluid. In incompressible flows ( $\rho=$ const.) it is possible to obtain the velocity component $u_{\perp}$ perpendicular to the hot-wire surface via the knowledge of the temperature difference $\Delta T$ between the probe and the fluid. This relation is described by King's law:

$$
\dot{Q}=\left[A+B\left(\rho u_{\perp}\right)^{1 / n}\right] \Delta T
$$

where $A, B, n$ are constants. In order to obtain the heat-loss $\dot{Q}$, the temperature difference $\Delta T$ is kept constant by fast resistance measuring bridges. Thereby the necessary electric power can be determined due to measuring the heating voltage. This method is known as Constant Temperature Anemometry (CTA). The output voltage $E$ of the CTA can be expressed as

$$
E^{2}=A^{*}+B^{*} u_{\perp}^{1 / n}
$$

\footnotetext{
${ }^{1} \mathrm{~A}$ detailed description can be found in (Eckelmann 1997).
} 
where the constants $A^{*}, B^{*}, n$ can obtained by calibration. This was done before and after each profile-measurement to compensate for any drift in temperature during the measurement. The hotwire has been positioned near a Prandtl tube outside the boundary layer to determine the free-stream velocity $U_{\infty}$ which is equal to $u_{\perp}$ provided proper orientation of the probe. The output voltage of the CTA was measured for a series of different free-stream velocities in order to obtain a set of tupels $\left(E, u_{\perp}\right)$, which were fitted to equation (5.2) in a least-squares sense.

The adjustment of the movable hotwire probe, i.e. its orientation and its distance from the wall, has been checked by a telescope set up in the settling chamber. The probe-holder was mounted at the 3-axis-traversing mechanism outside the wind tunnel, which allows $1 \mu \mathrm{m}$ steps in the wall normal direction $y$ and $10 \mu \mathrm{m}$ steps in streamwise $(x)$ and spanwise $(z)$ direction.

Before the measurements of the spanwise traverses presented below, the wall-normal position of maximum amplitude of the TS-waves has been determined at $z=0 \mathrm{~cm}$. The traverse coordinates have been adjusted such that the deviation from the mean-velocity became a minimum.

In the present experiment the output signal of the Dantec 56C17 CTA-bridge was amplified and adjusted by subtracting an off-set voltage in order to exploit the dynamic range of the 12-bit analog/digital converter $( \pm 5 \mathrm{~V})$. The relevant frequency range extended from $0 \mathrm{~Hz}$ to $1000 \mathrm{~Hz}$, which is far below the frequency limit $100 \mathrm{kHz}$ of this CTA. The circuit board which carried out the AD/DA-conversion is equipped with $64 \mathrm{kB}$ memory per channel and therefore allows to record a signal of up to 32000 samples at a sampling frequency of $2 \mathrm{kHz}$. The anti-aliasing lowpass filters have been adjusted to the chosen sampling frequency. The recorded time-signals have been evaluated in the PC using the MATLAB-Software.

\subsection{The real-time signal processing unit}

The real-time control algorithms have been implemented on a conventional PC hardware, operated by a Intel Pentium III $550 \mathrm{MHz}$, to save costs in comparison to commercial DSP-Boards and furthermore to profit from the frantic progress in this field. For this reason Dipl. Phys. Gero Timann developed a computer code ${ }^{2}$ which allows performing the necessary digital processing on a $\mathrm{PC}$ in real-time. The graphical user interface of this tool enables the user to plot the adapted filter coefficients or their frequency responses during use and to save them immediately. Additionally, the GUI can be adapted to personal preferences in order to obtain a better overview of the program procedures and the relevant parameters. A trigger signal (TTL) by any kind

\footnotetext{
${ }^{2}$ PPDSP, Pentium Pro Digital Signal Processing
} 
of hardware can initiate program procedures via the printer port of the $\mathrm{PC}$ and vice versa.

The programs of the adaptive filter algorithms have been assembled from two parts. The first part contained optimized assembler routines for the frequently used standard operations that are essential for real-time signal processing, like scalar product or vector gradient product. A detailed description of these routines ${ }^{3}$ (which belong to the standard of the so-called BLAS1-library) can be found on the Intel website (http://www.intel.com/software/products/mkl/index.htm). The second part can be seen as the composition of these routines to the algorithms, as well as the measurement procedures and the necessary input/output handling.

The DA/AD-conversion needed in the real-time processing has been carried out on a Crystal CS 4215 interface circuit board connected to 2 Fiji soundcards in the PC. Up to 16 DA/AD-channels can be operated by this device, simultaneously ${ }^{4}$. The signals that were processed by the real-time signal processing unit (e.g. the reference signals, the error signals, the signal of the moving hot-wire probe, the microphone signal etc.) were amplified for proper utilization of the 16-bit dynamic range $( \pm 0.1 \mathrm{~V})$ of the AD-converters. The wall hot-wire sensor signals were obtained as the output of Dantec 56C17 CTA-bridges.

Concerning the causality constraint (section 3.4) a problem arose due to the use of the Codec chips which cause a group delay of 38 samples per AD/DA-conversion by their internal filters (Evert 2000). Even though the group delay in milliseconds can be reduced by using a higher sampling frequency $f_{s}$, the remaining time for the computation will also decrease. Therefore a down\&up-sampling scheme was introduced to change the sampling rate, based on decimation and interpolation described e.g. in Oppenheim and Schafer (1999). With this scheme the sampling rate for the Codecchips have been 10 times higher than the actual data rate, which led to a reduction of the group delay to 8 samples.

Additionally, an 8-channel real-time FFT analyzer COMPASS by Gould Nicolet was used to analyze the measured signals. Especially the multiple coherence functions relevant for the multiple sensor configuration could be analyzed comfortably.

\footnotetext{
${ }^{3}$ SDOT, SAXPY etc.

${ }^{4}$ The interface card and the necessary low-level drivers have been developed and assembled in the electronics lab of the Third Physical Institute of the Georg-August University Göttingen
} 


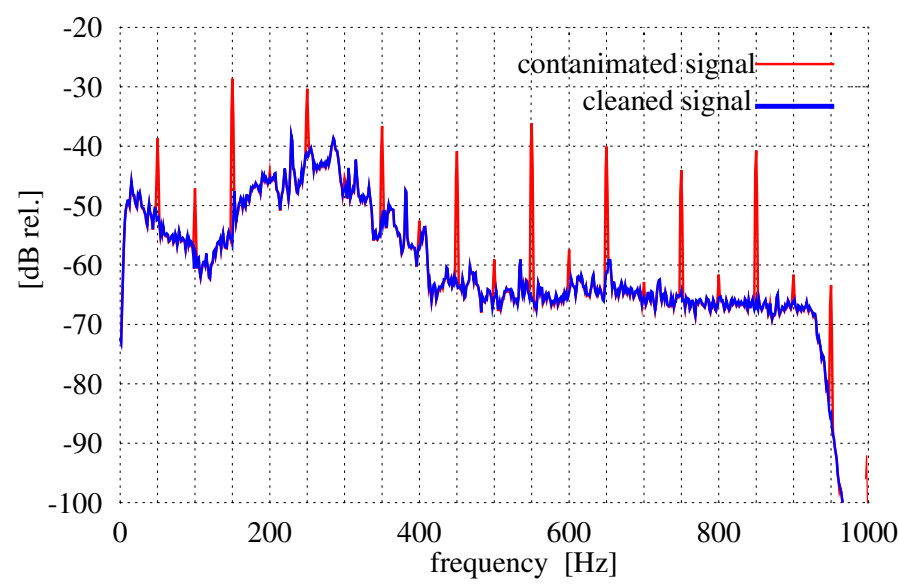

Figure 5.3: Spectrum (PSD) of a typical hot-film sensor signal showing the reduction of electrically induced noise by adaptive filtering.

\subsection{Digital reduction of uncorrelated electric con- tamination of the signals}

The contamination of the signals by induced electrical noise containing $50 \mathrm{~Hz}$ and its harmonics can cause problems in realizing an AWC system. If this electrically induced noise is present in the reference and error signals, the actuators will excite a counter-phase $50 \mathrm{~Hz}$ TS-wave to reduce the electrical noise correlation. This disturbs the desired cancellation process of flow instabilities.

Different efforts concerning the improvement of the analog signals have been successful, but could not fully solve this problem. Therefore the contamination has been reduced by means of adaptive filters. Hereby a transformer is used to obtain a nonsymmetric square-signal with fundamental frequency of $50 \mathrm{~Hz}$ and all of its harmonics. This signal is the reference signal $x[n]$ of the adaptive filter and the contaminated measured signal serves as the desired signal $d[n]$. The LMS-algorithm decorrelates these signals and therefore the remaining error signal $e[n]$ will be 'cleaned' from the induced electrical noise that is correlated to the reference signal. The efficiency of this method is illustrated by Fig. 5.3, which shows the spectrum of a hotfilm reference sensor, with and without the electric noise reduction (ENR) applied. 


\section{Chapter 6}

\section{Development of the active wave cancellation device}

The design of the active wave cancellation device has been a process that was determined by the actual knowledge of the demands to such a device (see chapter 3).

\subsection{Preliminary cancellation device (Type I)}

This section describes the design of the Type I AWC device and summarizes the results obtained with this device. These results showed some unexpected effects leading to improvements realized in the Type II AWC device which is investigated in more detail in the following chapters.

In order to reduce the complexity of the cancellation device by the usage of only a small number of sensors but still providing a sufficient characterization of the incident wave field a suitable spanwise distance of the sensors had to be chosen. A former investigation of the spanwise coherence of naturally occurring TS-waves using the same set-up determined the averaged spanwise coherence length, defined by a $50 \%$ decrease, for slightly varying streamwise pressure gradients as $9 \mathrm{~cm}$ (Sundaram et al. 1997). Based on this result the spanwise distance was chosen to be $10 \mathrm{~cm}$.

TS-waves have been measured at a free-stream velocity of $20 \mathrm{~m} / \mathrm{s}$ at a streamwise position of about $x=40 \mathrm{~cm}$. A SNR of about $15 \mathrm{~dB}$ has been observed in the obtained spectra. This streamwise position has been chosen for the reference sensors.

In order to comply with the causality constraint for this flow velocity, the streamwise distance between the reference sensors and the actuators had to be above a certain limit determined by the minimum time TS-waves need for propagation. Based on the maximum group velocity of the TS-waves, known as about $45 \%$ of the free-stream 
velocity, and the experience by former experiments (Evert 2000), the distance was chosen to be $26 \mathrm{~cm}$. These considerations resulted in the following sensor configuration with a Nyquist-wavenumber of $\beta_{N y}=0.314 / \mathrm{cm}: 4$ reference sensors were placed at $x=40 \mathrm{~cm}, z=-15,-5,+5,+15 \mathrm{~cm}$ and 4 error sensors were placed at $x=72 \mathrm{~cm}, z=-15$, $-5,+5,+15 \mathrm{~cm}$. The actuator array was mounted at a streamwise position $x=66 \mathrm{~cm}$ (slot). In the following this configuration is referred to as Type I-configuration.

Concerning the design of suitable actuators for an active wave cancellation device a number of requirements have to be met (see chapter 3 ).

First, the actuators must generate a reasonably flat disturbance spectrum in the relevant frequency range, because the control algorithm has to invert the transfer function of the actuators.

In order to generate vertical velocity fluctuations in the boundary layer a loudspeaker connected via a plastic tube to a slotted chamber has been used very often. Such an arrangement similar to the one of the 2D-disturbance generator can lead to strong resonances in the produced disturbance spectrum. The 2D-disturbance generator which is of this type generates resonance-peaks of up to $20 \mathrm{~dB}$ measured by a microphone $1 \mathrm{~mm}$ above the slot.

These resonance effects have been reduced by the direct implementation of the loudspeakers into the plate and thereby avoiding the plastic-tube-chamber system. This led to the design of the actuator sketched in Fig. 6.1. The actuator had to be a slim construction in order to fit into the $4 \mathrm{~cm}$ thick plate to avoid any additional blockage of the wind tunnel. A set of 4 loudspeakers has been built into an aluminium box each into a separate chamber. Monacor SPF 50 miniature loudspeakers were used due to their extremely small mounting depth. The chamber volume was $3 \times 100 \times 50 \mathrm{~mm}^{3}$.

The second requirement that has to be met by the actuators is that they only generate disturbances having a spanwise wavenumber spectrum such that the disturbances can be sufficiently sampled by the error sensors without aliasing. In order to smooth out the spanwise disturbance distribution the chambers were connected to the surface of the plate by a shared slit. Due to the limited space available for this device the depth of the $0.3 \mathrm{~mm}$ wide slit was limited to the maximum of $6 \mathrm{~mm}$. However, later this precaution turned out to be insufficient, which led to a rather broad wavenumber spectrum of this actuator. The measured spectrum is presented in the following section (Fig. 6.10).

Furthermore, this type of actuator had to show that it can be used to generate disturbances of an amplitude comparable to the one of the pressure chamber-tube actuators. Therefore microphone measurements without flow have been made $1 \mathrm{~mm}$ above the slot to estimate the normal velocity fluctuations via the moving media mass (Raguse 1998). The obtained maximum velocity was $0.2 \mathrm{~m} / \mathrm{s}$ and therefore was comparable with the pressure chamber speakers used as disturbance generator and as actuator by Evert 




Figure 6.1: Actuator Type I: Schematic spanwise pressure distribution and simplified top view (left). A cross section of the actuator in streamwise direction, all dimensions in millimeters (right).

et al. (1999). An obvious improvement in the frequency response has been reached in comparison to the 2D-disturbance generator, i.e. it did not show any strong resonances except for a weak resonance in the relevant frequency range (100-500 Hz) with a $6 \mathrm{~dB}$ maximum.

The first active wave cancellation experiments have been carried out at various flow velocities using this Type I device. It turned out that the coherence $C_{x d}(f)$ between reference and error sensors which is essential for the success of active wave cancellation was rather small, at maximum $C_{x d}(f) \approx 0.91$. Attempts to increase the coherence by increasing the Reynolds number in order to improve the SNR of the sensor signals led to already intermittent signals of the error sensors.

In order to increase the coherence in the experiment described next, a small adverse pressure gradient has been imposed on the boundary layer.

The results shown in Fig. 6.2 have been obtained at a flow velocity of $U_{\infty}=15 \mathrm{~m} / \mathrm{s}$ and a small adverse pressure gradient. A SISO-AWC system consisting of the reference sensor, the actuator and the error sensor all at spanwise position $z=-5 \mathrm{~cm}$ has been operated in order to cancel 'natural' TS-waves (left side of Fig. 6.2) and 'artificial' TSwaves. The artificial TS-waves have been excited by the $2 \mathrm{D}$-disturbance generator in addition to the present natural TS-waves. The disturbance generator has been driven by a white noise signal in order to excite TS-waves at all frequencies in the considered frequency range $(0-1000 \mathrm{~Hz})$.

The spectra of the error sensor are shown for both cases in Fig. 6.2 (a) \& (b). One can distinguish the spectra for the cases 'with AWC', 'without AWC' and 'the- 


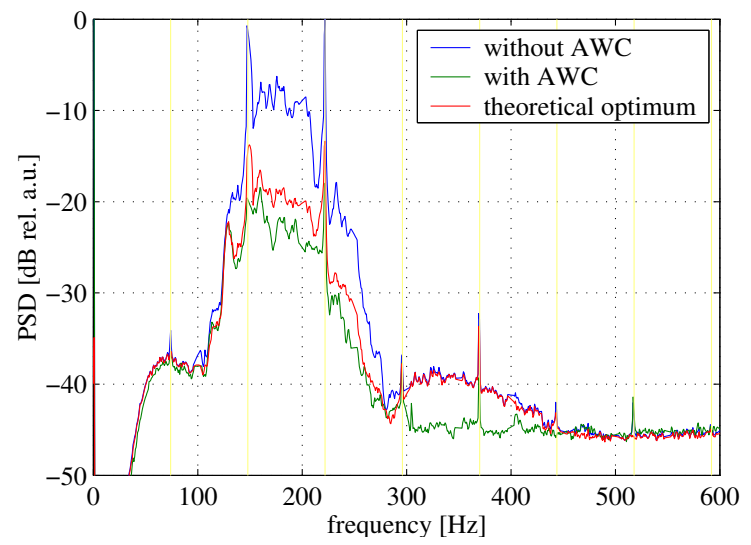

(a) Error sensor spectra with and without AWC.

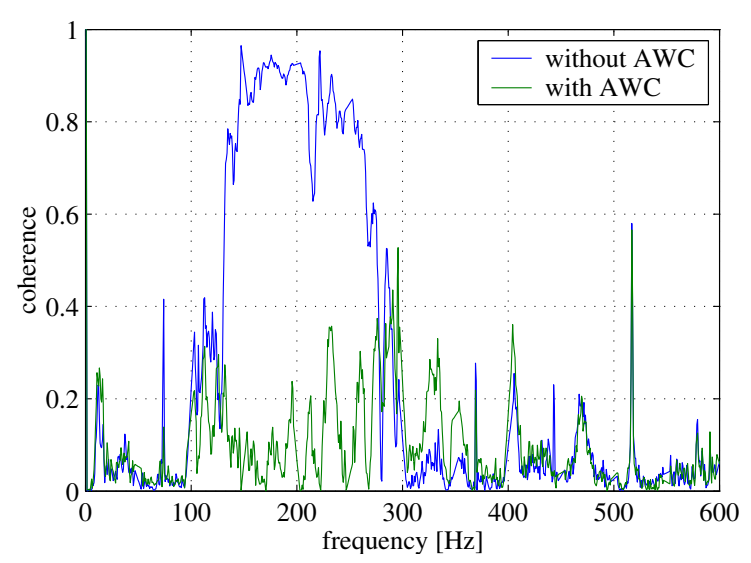

(c) Coherence between reference and error sensor, with and without AWC.

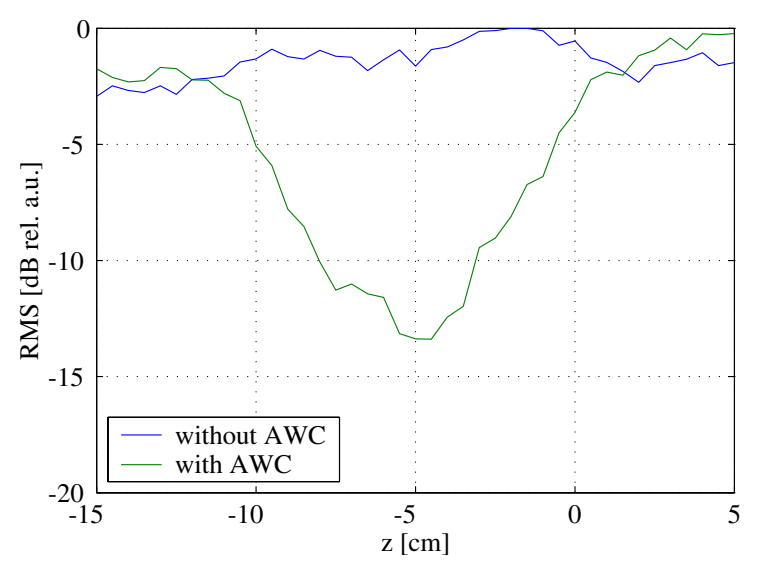

(e) Spanwise RMS-distribution (100-260 Hz) at $x=72 \mathrm{~cm}$, with and without AWC.

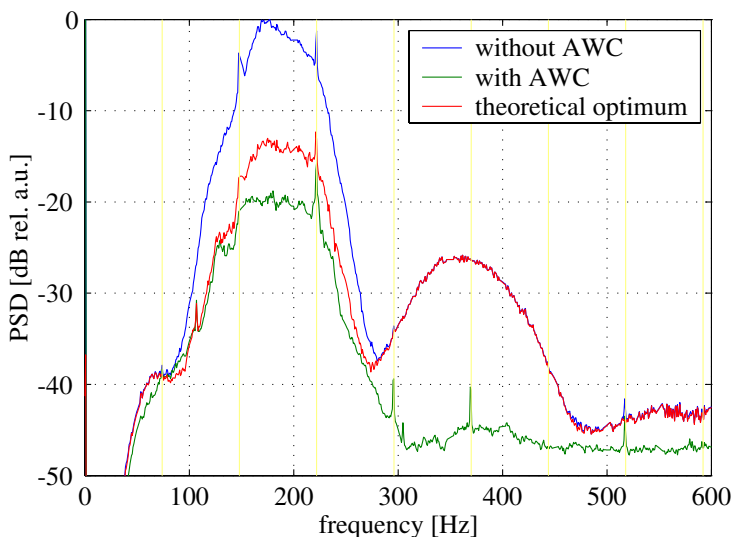

(b) Error sensor spectra with and without AWC.

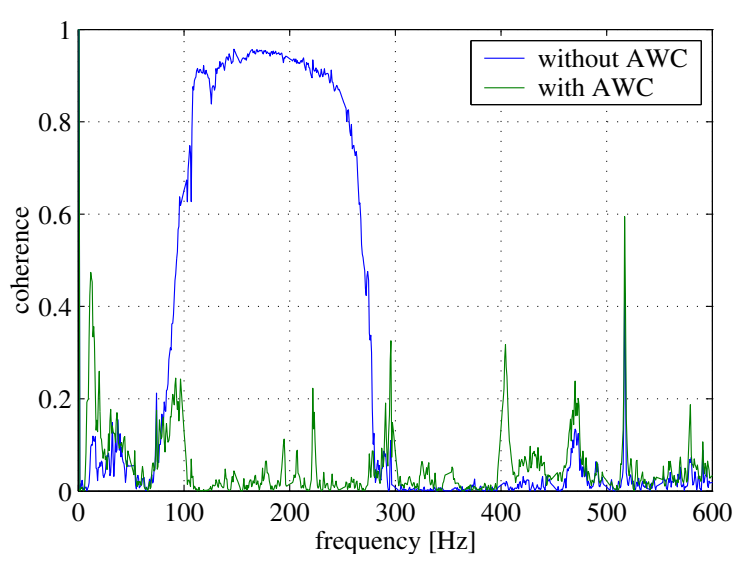

(d) Coherence between reference and error sensor, with and without AWC.

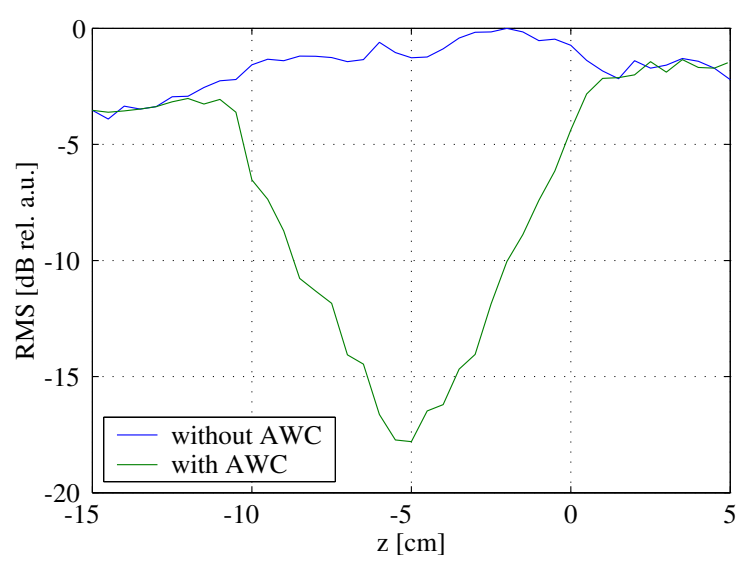

(f) Spanwise RMS-distribution (100-260 Hz) at $x=72 \mathrm{~cm}$, with and without AWC.

Figure 6.2: Comparison of SISO AWC system applied to 'natural' (left side) and 'artificial' (right side) TS-waves. 
oretical optimum'. The latter are determined by equation (4.32) from the measured streamwise coherence between the reference and error sensor without AWC shown in Fig. 6.2 (c) \& (d).

In both cases, the spectra indicate a higher reduction than expected from the 'theoretical optimum'. The reason for this has been found in nonlinear effects caused by the initially high amplitude of the TS-waves measured at the error sensors. This leads to a degradation of the streamwise coherence. By switching on the cancellation device the amplitude of the TS-waves is reduced at the streamwise position of the actuator such that the influence of the occurring nonlinearities will be decreased as well. Therefore the decisive measure to evaluate the reduction potential of the AWC system would be the coherence between the reference sensor and the actuator. A priori the latter is not available, therefore the coherence between the reference and error signal has been considered.

For the case of natural TS-waves as well as for the one of artificial TS-waves reductions of up to $20 \mathrm{~dB}$ have been obtained. In the first case the high reduction is achieved only for a single frequency component whereas in the second case the reduction is higher and spread over the whole frequency range of the fundamental TS-waves. It is obvious that the smoothness of the spectra depends on the frequency distribution of the excitation process. In the TS-frequency range the spectra 'with AWC' show in both cases remainders of about $20 \mathrm{~dB}$ above the noise level indicating a further reduction potential. Surprising is the fact that in the cases of natural TS-waves the absolute level of the remaining disturbances is lower than for artificial TS-waves.

The strong single frequency components visible in the spectra (marked by the yellow lines in Fig. 6.2 (a) \& (b)) are the blade-passing frequencies of the rotor. Similar to the electrical induced noise components (see section 5.4) these components have first been considered as artifacts and therefore have been removed from the sensor signals by means of adaptive filtering. For this purpose the reference signal was obtained by an inductive indicator that was mounted at the wall opposite of the rotor. Later, it turned out that the associated pressure fluctuations generate TS-waves and therefore have to be considered in the AWC. However, these frequency components have been removed from the figures presented in the following chapters for the sake of clarity.

The nonlinear effects during the propagation of the TS-waves cause the "harmonic" part of the spectra. This part is eliminated by the AWC even though only linear transfer functions are considered. The AWC system reduces the linearly correlated part of the spectrum and therefore the amplitude depending generation of the harmonic part is suppressed as well. A detailed study of nonlinear effects in the propagation of TS-waves and the consequences for an AWC is given by Evert (2000).

The coherence between the reference and the error sensor (see Fig. 6.2 (c) \& (d)) is reduced quite well for both cases due to the action of the cancellation system. Never- 


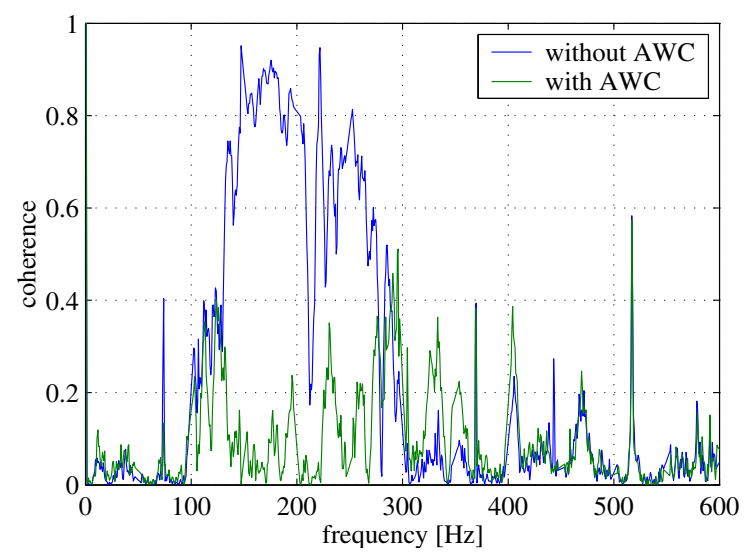

(a) Coherence between the neighboring reference sensor at $z=-10 \mathrm{~cm}$ and the error sensor.

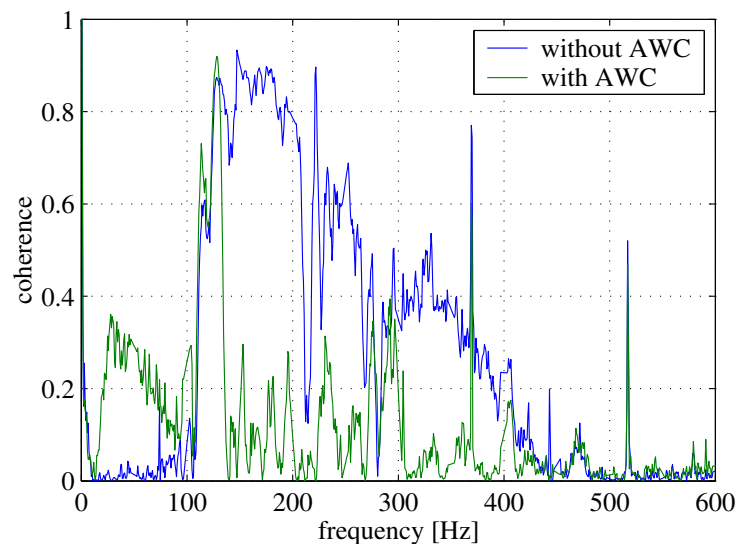

(b) Coherence between two neighboring error sensors $(z=-5,+5 \mathrm{~cm})$.

Figure 6.3: Lateral correlations of 'natural' TS-waves with and without the control by the SISO AWC system.

theless there are some remainders for certain frequencies that are higher for the case of 'natural' TS-waves. The origin of these effects will be discussed in chapter 9 .

The spanwise extent of the AWC is indicated in Fig. 6.2 (e) \& (f) for both cases. The figures show the spanwise RMS-distribution (100-260 Hz) that was measured by a hot-wire traversed $2 \mathrm{~cm}$ downstream of the error sensor. For the natural TS-waves the maximum reduction is about $12 \mathrm{~dB}$ whereas for artificial TS-waves $16 \mathrm{~dB}$ are achieved. In both cases the affected region can be characterized by a drop of the achieved reduction to $3 \mathrm{~dB}$ within a lateral distance of $5 \mathrm{~cm}$. Furthermore, in the case of natural TS-waves a small amplification is observed at spanwise distances greater than $7 \mathrm{~cm}$ from the error sensor. The discussion of this effect will also be postponed to chapter 9.

The potential of an additional consideration of lateral correlation in the AWC system can be estimated by the lateral correlations shown in Fig. 6.3 (a \& (b).

Fig. 6.3 (a) shows the coherence between the neighboring reference sensor $(z=-$ $10 \mathrm{~cm}$ ) and the error sensor used in the SISO-AWC system. It becomes obvious that this coherence is already reduced to low level by the AWC. The remaining part looks very similar to the remainder of the coherence in streamwise direction (Fig. 6.2 (c)).

Therefore one may think that no significant improvement is expected for the consideration of these correlations in AWC system. This conclusion cannot necessarily be drawn because the SISO-AWC system may reduce the correlations caused by 3D waves 


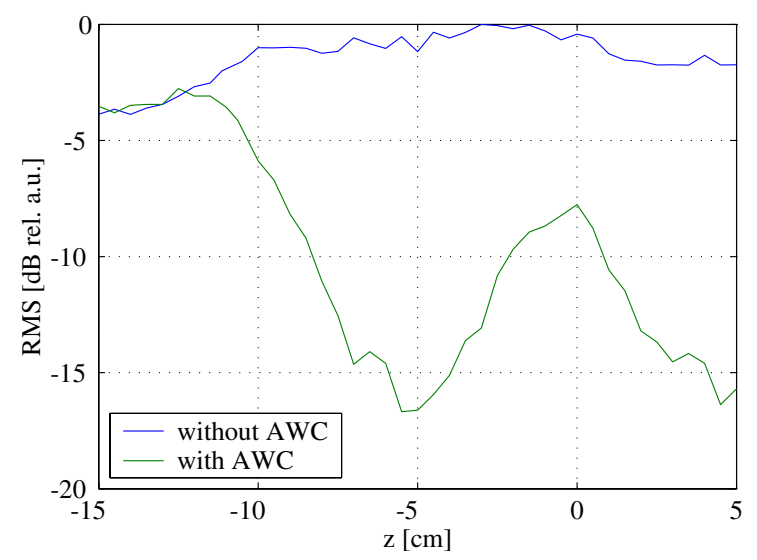

Figure 6.4: The spanwise RMS-distribution (100-260 Hz) of two spanwise independent SISO-AWC systems applied to 'artificial' 2D TS-waves (measured at $x=74 \mathrm{~cm}$ ).

locally at the error sensor by the generation of 2D-waves. This would lead to an increased remainder of the disturbances in between neighboring error sensors. However, this assumption cannot be clarified by the available data.

The action of the AWC also reduces the coherence between the neighboring error sensors at $z=-5,+5 \mathrm{~cm}$, shown in Fig. 6.3(b). However, there is one part of the coherence that is increased by the AWC system, the subharmonic part. This effect turned out later to be caused by the excitation process of the slot-actuator, this has also been observed in other studies (Sturzebecher and Nitsche 2002). Also remarkable is the high coherence between 110 and $130 \mathrm{~Hz}$ that has not been affected by the AWC.

In order to test whether a spanwise chain of SISO-AWC systems are sufficient in the case of a 2D dominated wave field, two independent SISO-AWC systems have been lined-up in spanwise direction and applied to artificial TS-waves excited again by the $2 \mathrm{D}$ disturbance generator. The spanwise RMS-distribution obtained $2 \mathrm{~cm}$ downstream of the error sensors is shown in Fig. 6.4. Whereas a reduction of $16 \mathrm{~dB}$ is obtained at the spanwise position of the error sensors $(z=-5,+5 \mathrm{~cm})$ only $7 \mathrm{~dB}$ of reduction are achieved in between. The origin of the remainders in between the sensors has not been fully understood. It is likely that one part of it is caused by $3 \mathrm{D}$ waves that have not been affected due to insufficient sampling. Another part may have been caused by the effect described above, that 3D waves are cancelled locally at the error sensors resulting in an enhancement of the remainders between the sensors.

A $2 \times 2$ MIMO system has also be applied but no significant difference has been observed. This may be explained by the inappropriate controllability of the used actuators, namely that these actuators were not capable to control the disturbances at the neighboring error sensors, as turned out later. 


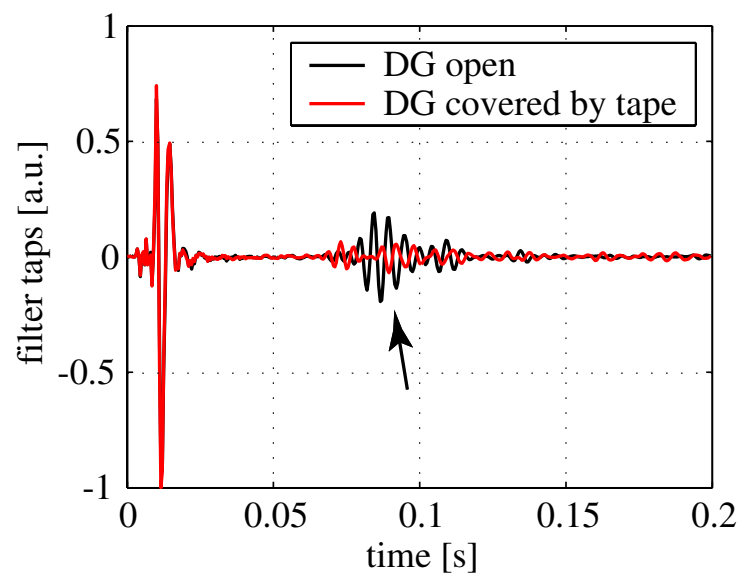

(a) Impulse response



(b) Magnitude of the transfer function $\mathbf{S}$

Figure 6.5: The influence of the roughness caused by the disturbance generator (DG) slot on the estimation of $\mathbf{S}, \Delta x_{a e}=6 \mathrm{~cm}$.

Furthermore an interesting effect has been observed in the modelled secondary path transfer functions $\mathbf{S}$ between the actuator $(z=-5 \mathrm{~cm})$ and the error sensor $(z=-5 \mathrm{~cm})$. It has been modelled by a larger number of filter taps (512) than needed for the AWC. The system identification scheme has been applied feeding the actuator by a white noise signal while an adaptive filter modelled the transfer function to the error sensor. A typical estimate of the secondary path transfer function in streamwise direction is shown in Fig. 6.5 (a). The impulse response represented by the filter taps shows beside the expected TS-pulse at a delay of 0.01 seconds also a second smaller pulse that arrives later ( 0.085 seconds) at the error sensor. Latter corresponds to the pulse propagation time between the disturbance generator, which can be estimated by the average group velocity of TS-waves known as about $40 \%$ of the free-stream velocity $\left(U_{\infty}=16 \mathrm{~m} / \mathrm{s}\right)$. Covering the slots of the disturbance generators (both, 2D \& 3D) by $0.1 \mathrm{~mm}$ thin tape reduces the pulse by about a factor of 3 .

Fig. 6.5(b) shows the magnitude of the transfer functions and indicates the interference pattern in the TS-frequency range which is decreased by taping the disturbance generators.

It turned out that the actuators also generate sound that travels upstream and excites TS-waves at the roughness of the disturbance generator slot. The remaining part, after taping the slots, is likely to be caused by the roughness from the edges of the tape. 


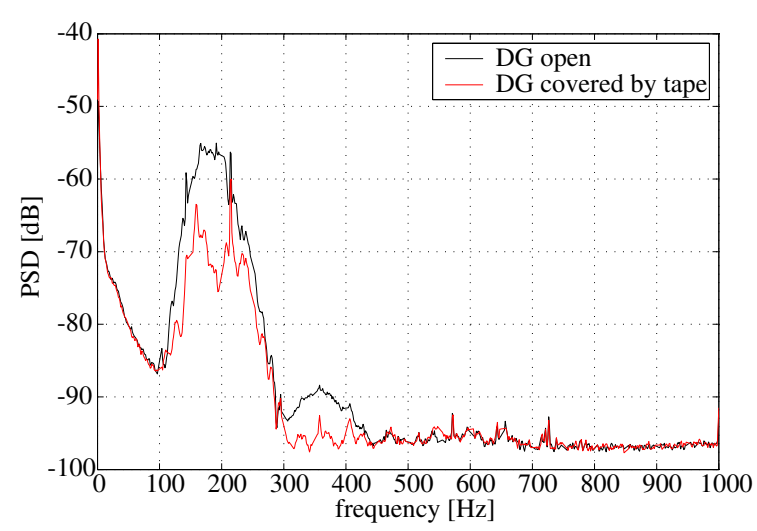

(a) Spectra of the error $\operatorname{sensor}(z=-5 \mathrm{~cm})$.

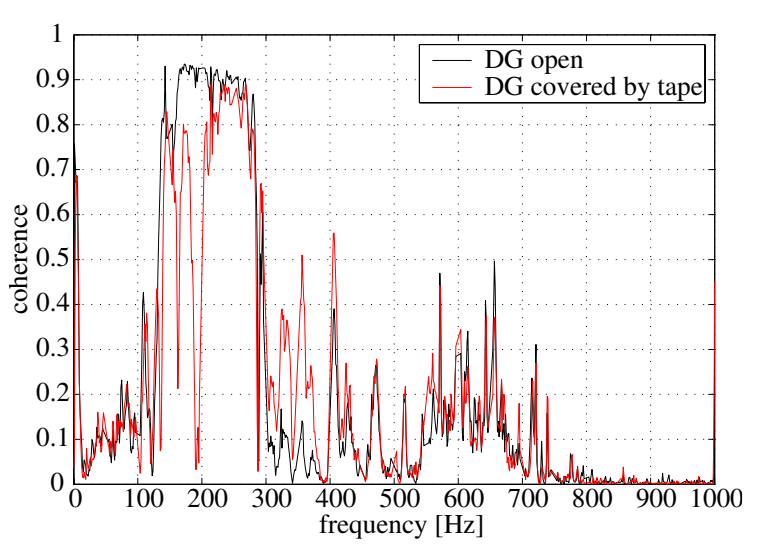

(b) The streamwise coherence between the reference and the error sensor.

Figure 6.6: Spectra and coherence measurements indicating the receptivity of the disturbance generator slot.

Furthermore, it becomes visible in Fig. 6.5 (b) that the disturbance spectrum of the actuator measured at the error sensor is still rather broadband and has not been shrunk to the TS-frequency band within the downstream distance of $6 \mathrm{~cm}$.

The acoustic receptivity of the disturbance generator slot also significantly influences the 'natural' disturbance spectrum. This can be concluded from the spectra and the streamwise coherence shown in Fig. 6.6. The error sensor spectrum is reduced by about $15 \mathrm{~dB}$ and the coherence decreases significantly as well. The increase of the coherence in the frequency range of $300-400 \mathrm{~Hz}$ can be explained by the vanishing of the nonlinear part in the error sensor signal.

What are the main conclusions from these experiments with the preliminary cancellation device concerning the design of an improved cancellation device?

- The Type I actuator shows an improved frequency response in comparison to tube-chamber actuators.

- Due to the large streamwise distance between the reference sensor and the actuator $(26 \mathrm{~cm})$, and the error sensor $(32 \mathrm{~cm})$ the degradation of the coherence is also quite high, mainly caused by nonlinearities.

- The 'natural' TS wave field is mainly generated by the acoustical noise in the wind tunnel. Furthermore, it is dominated by 2D components excited at the 2D roughness of the disturbance generator slots. 
- The spanwise distance $(10 \mathrm{~cm})$ between the sensors and between the actuators is too large to sufficiently sample and reconstruct the relatively weak 3D part of the disturbance spectrum.

- The actuators emit also sound that may generate TS-waves at upstream roughnesses.

- The streamwise distance between the actuators and the error sensors is probably too small to avoid any near field effects.

- The actuator generates a broad spanwise wavenumber spectrum that cannot be sampled sufficiently by the error sensors. The related spanwise spreading is too small to control the disturbances at neighboring error sensors (explained in the following section).

\subsection{Improved cancellation device (Type II)}

To overcome these problems a number of changes had to be made leading to a new sensor-actuator configuration (Type II).

In order to minimize the enforcement of $2 \mathrm{D}$ components in the natural TS-wavefield by the $2 \mathrm{D}$ roughness of the disturbance generators, these have been covered by tape. The edges of the tape have been smoothed out by a modelling paste in order to minimize the influence on the boundary layer and the acoustic receptivity. The disadvantage of this is that also the measured amplitudes of the natural TS-waves will be significantly smaller.

A spanwise $3 \mathrm{~mm}$ wide opening has been cut into the tape above the center slot of the 3D disturbance generator in order to use it as a 'point-source'.

In order to counteract the decrease in amplitude of natural TS-waves, the reference sensors have been placed $11 \mathrm{~cm}$ further downstream. Furthermore the hot-film sensors have been replaced by wall hot-wire sensors developed and manufactured at the TUBerlin (see Fig. 6.7). They have been found to improve the SNR by up to $10 \mathrm{~dB}$ due to the reduction of the heat transfer to the wall. This is achieved by spanning the hot-wire in a small cavity at the same level as the surface of the wall. Moreover these sensors can be operated with a higher overheat ratio than common hot-film sensors, which in the present work was chosen to be $\frac{R}{R_{0}}=1.7(R$ : the electric resistance of the overheated sensor, $R_{0}$ : the electric resistance at fluid temperature). The sensors have been operated in constant-temperature-modus (Dantec 56C17 CTA-bridge). The output signal is here a function of the wall shear stress $\tau_{W}$ (Baumann 1999; Eckelmann 1997). 


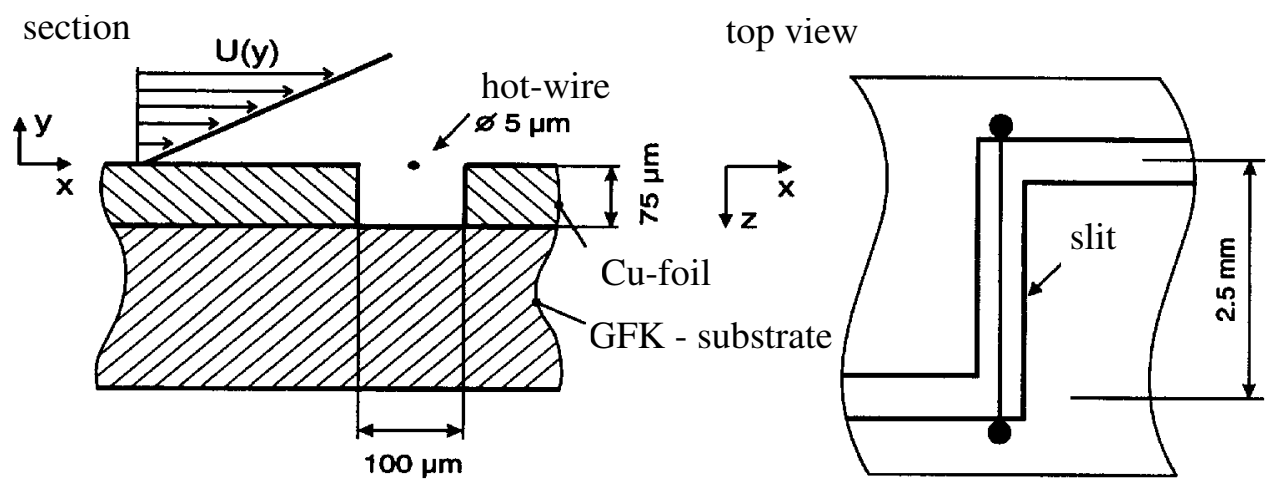

Figure 6.7: Wall-hot wire sensor as described in Baumann (1999).

In order to improve the spatial sampling of the disturbances, the spanwise distance of the sensors was halved, which leads to the Nyquist-wavenumber of $\beta_{N y}=0.628 \mathrm{~cm}^{-1}$. Also the streamwise distance between the reference sensors and the actuators was shrunk to improve the streamwise coherence.

Moreover the streamwise distance between the actuators and the error sensors was increased by $5 \mathrm{~cm}$ to reduce near-field effects.

These changes led to the following Type II configuration: 8 reference sensors placed at $x=50 \mathrm{~cm}, z=-17.5,-12.5,-7.5, \cdots,+17.5 \mathrm{~cm}$ and 8 error sensors placed at $x=72 \mathrm{~cm}$, $z=-17.5,-12.5,-7.5, \cdots,+17.5 \mathrm{~cm}$. The new position of the actuator slot was chosen to be $x=61 \mathrm{~cm}$ (see Fig. 6.8).

In order to check the wavenumber spectrum of the actuator, the directional characteristics have been measured at the streamwise location of the error sensor. This has been achieved by measuring the transfer functions to a hot-wire signal at various spanwise positions $(\Delta z=-10 \mathrm{~cm}$ to $+10 \mathrm{~cm})$. This procedure is described in section 7.2 in more detail. The obtained wavenumber spectrum of the Type I actuator is plotted in Fig. 6.10 (a). The contour-lines indicate the magnitude of the frequency response in the plane spanned by the dimensional frequency $f$ and the spanwise wavenumber divided by the Nyquist wavenumber of the sensor configuration (Type I) $\frac{\beta}{\beta_{N y}}$.

It shows a strong center maximum for $\left|\frac{\beta}{\beta_{N y}}\right| \leq 1$ in the amplified frequency range but there is only a small decrease of amplitude till the Nyquist wavenumber. Furthermore there are two side-lobes at $\frac{\beta}{\beta_{N y}} \approx \pm 3.1$ of a $10 \mathrm{~dB}$ smaller amplitude $^{1}$ that can be explained by the insufficient smoothing of the spanwise disturbance distribution. This behavior is similar to that of a sinc-function which is the Fourier transform of

\footnotetext{
${ }^{1}$ Not visible in the figure are further side-lobe maxima at $\frac{\beta}{\beta_{N y}} \approx \pm 6.3$.
} 

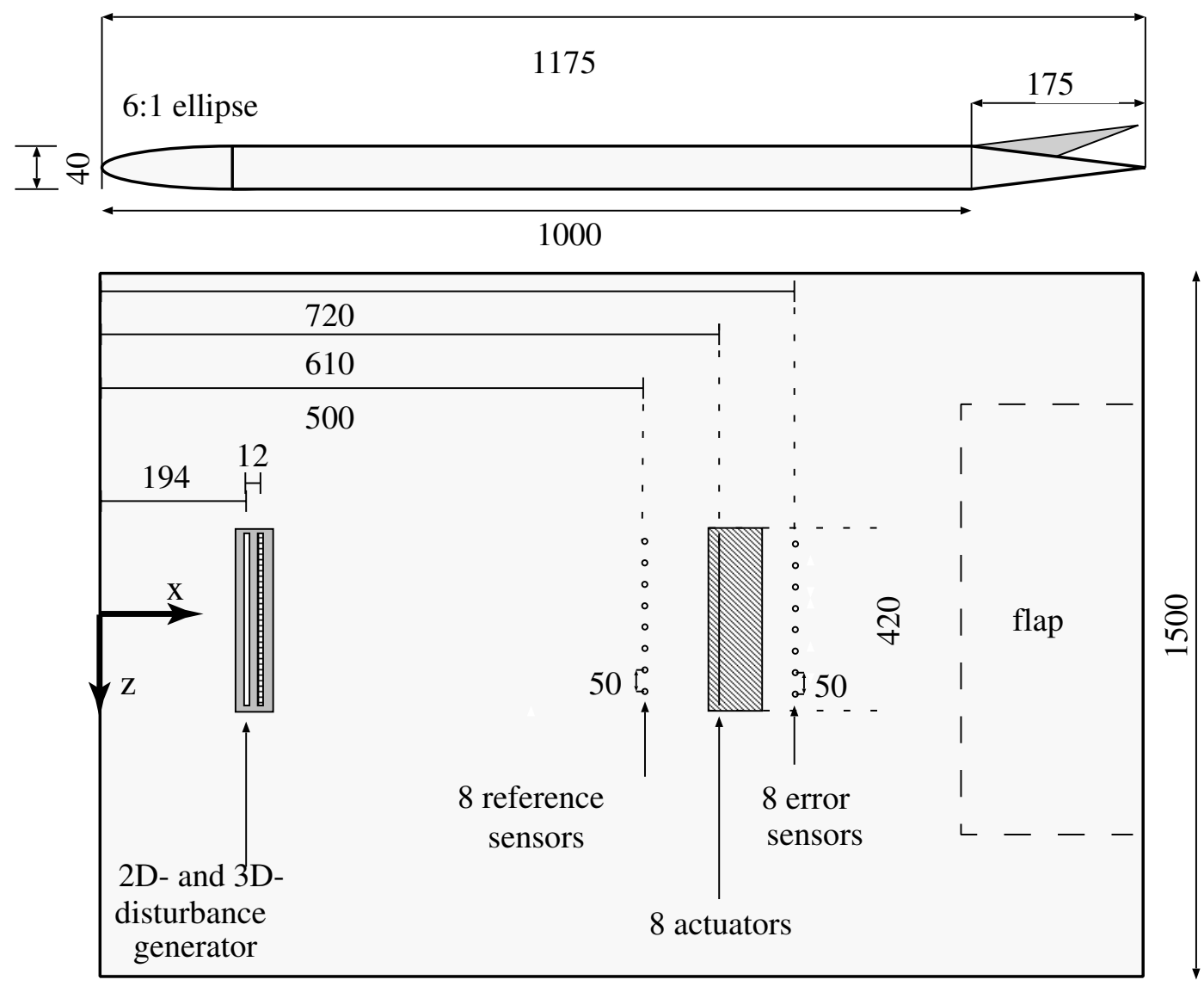

Figure 6.8: Side view and top view of the plate including the cancellation device (Configuration Type II), all dimensions are given in millimeters.

the rect-function. The latter is illustrated in Fig. 6.1 by the sketched spanwise disturbance distribution of rectangular shape. In real space this insufficient smoothing of the excitation distribution leads to cancellation waves that are concentrated in spanwise direction such that neighboring error sensors will not be affected much by them. Hence, the secondary path transfer matrix $\mathbf{S}$ becomes close to a diagonal matrix. Thus, the Type I actuator turned out to be inappropriate for the usage in a wave cancellation device aiming to take into account lateral correlations of the disturbance wave field.

For this reason the actuator was redesigned in order to generated a smoother spanwise disturbance distribution, see Fig. 6.9 (b).

As for the sensors, the spanwise distance between the actuators has also been halved and therefore 8 loudspeakers (Monacor SPF 45) have been assembled in an aluminium box of the same dimensions as in the Type I configuration. Due to the restricted space 


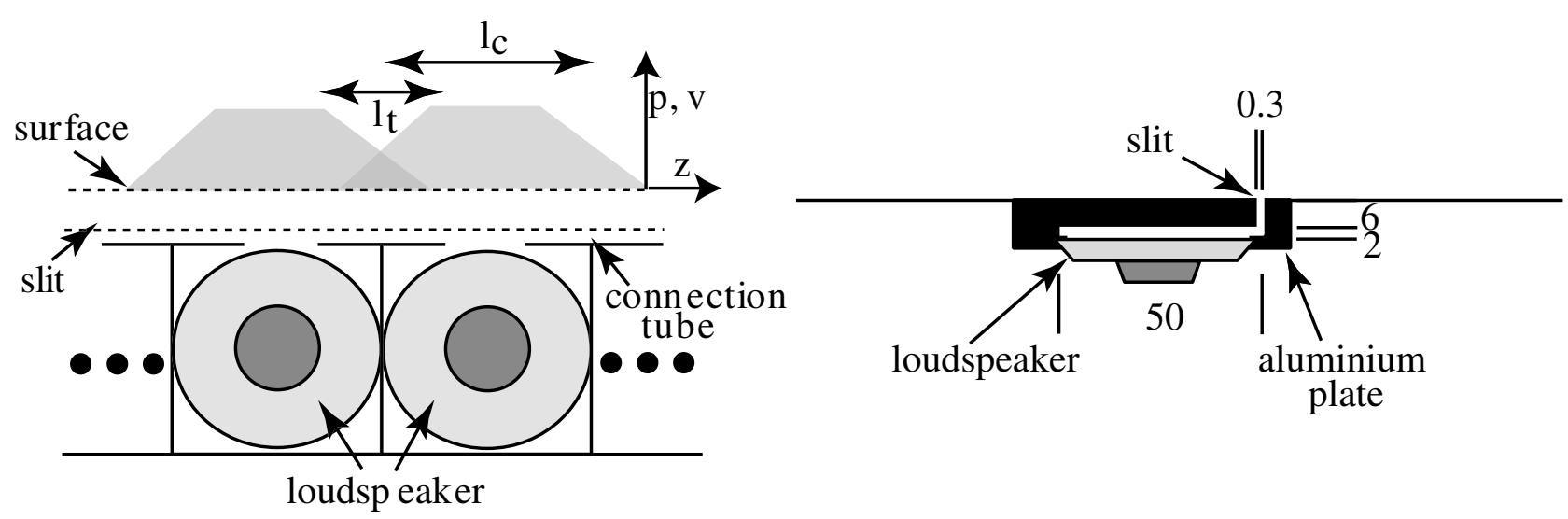

Figure 6.9: Actuator Type II: Schematic spanwise pressure distribution and simplified top view (left). A cross section of the actuator in streamwise direction, all dimensions in millimeters (right).

in the plate the depth of the slit could not be increased in order to achieve a smoother spanwise disturbance distribution. Therefore an additional connection between neighboring chambers was introduced. This has been modified by a wall, thereby forming a kind of square connection tube $(1 \times 2 \mathrm{~mm})$ which had an opening to the plate surface by the $0.3 \mathrm{~mm}$ slot.

If only one actuator is driven, a pressure difference between both ends of the connection tube will generate an oscillating flow. If the area of the slot is small in comparison to the cross-section area of the connection-tube, the pressure will decrease linearly. The resulting spanwise disturbance distribution can be described by the convolution of two rect-functions, one defined by the chamber width $l_{c}$ and the other one by the length of the slotted tube $l_{t}$. The trapezoidal form of the spanwise distribution (sketched on top of Fig. $6.9(\mathrm{a}))$ is obtained due to this convolution. The wavenumber spectrum of the disturbance is therefore obtained by the product of the two Fourier transforms, namely two sinc-functions. The ratio $\frac{l_{c}}{l_{t}}$ has been optimized in order to reduce higher wavenumber components. The best result was obtained for $\frac{l_{c}}{l_{t}}=0.5$, which leads to the cancellation of the first side lobe of the chamber sinc-function by the first zero of the tube sinc-function. Due to halving of the spanwise distance between the sensors the number of actuators was also doubled by halving their spanwise extent. This led to the spanwise extent of each actuator of $5 \mathrm{~cm}$ and thereby to an optimum length of the connection tube of $2.5 \mathrm{~cm}$.

In order to compare the wavenumber spectrum of this Type II actuator with the one of the Type I actuator again frequency responses have been measured for different spanwise positions at the same streamwise distance $(6 \mathrm{~cm})$ as before and for a similar 


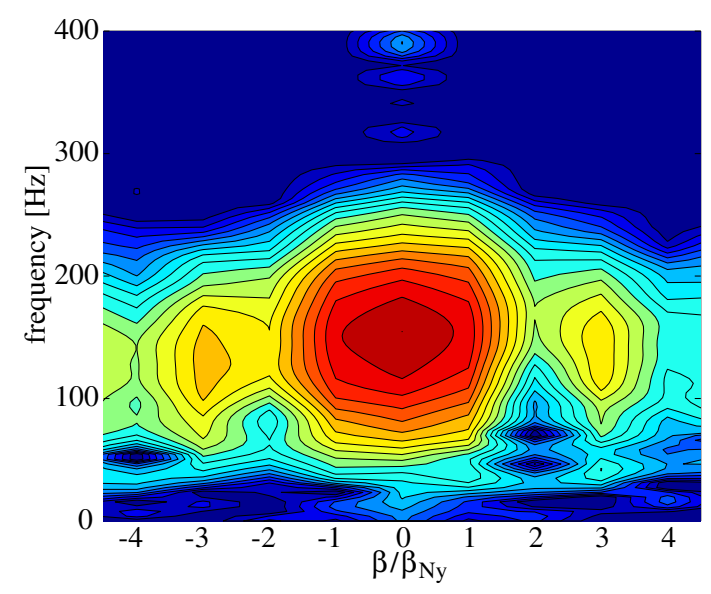

(a) Type I actuator, $\Delta \mathrm{x}=6 \mathrm{~cm}$, $U_{\infty}=16 \mathrm{~m} / \mathrm{s}$.

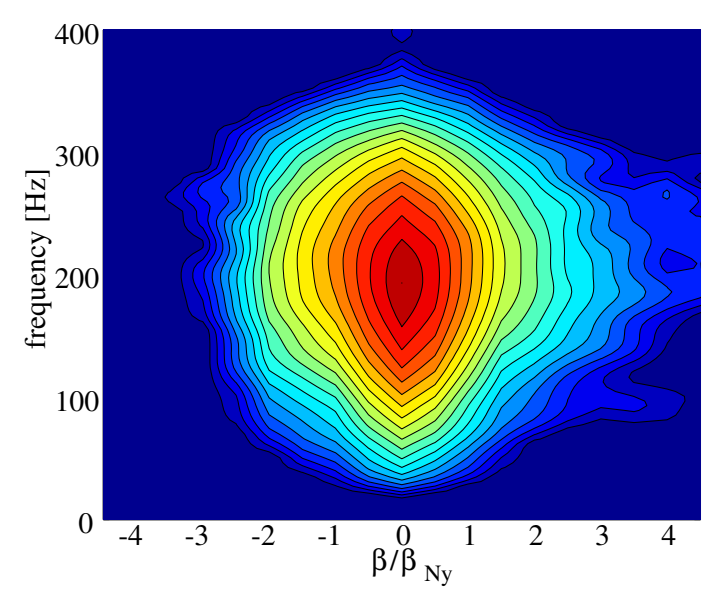

(c) Type II actuator, $\Delta \mathrm{x}=11 \mathrm{~cm}$, $U_{\infty}=18 \mathrm{~m} / \mathrm{s}$.



(b) Type II actuator, $\Delta \mathrm{x}=6 \mathrm{~cm}, U_{\infty}=16 \mathrm{~m} / \mathrm{s}$

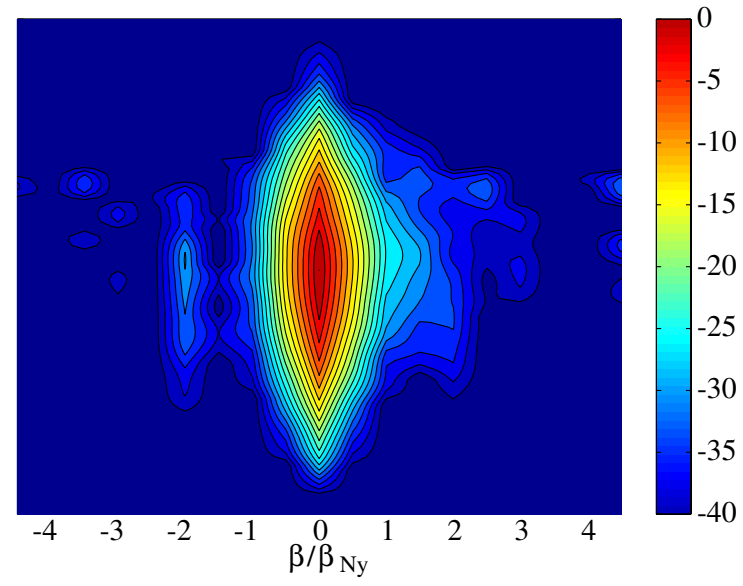

(d) Pseudo actuator, $\Delta \mathrm{x}=11 \mathrm{~cm}, U_{\infty}=18 \mathrm{~m} / \mathrm{s}$

Figure 6.10: Spatial frequency spectra for the different actuators. The spanwise wavenumber is divided by the Nyquist wavenumber of the sensor configuration and the colors indicate the logarithmic scale, given in decibels.

excitation amplitude. The obtained wavenumber spectrum is plotted in Fig. 6.10 (b). It shows obvious improvements in comparison to the Type I actuator such that the main part of the spectrum is approximately confined to the Nyquist-wavenumber range. 


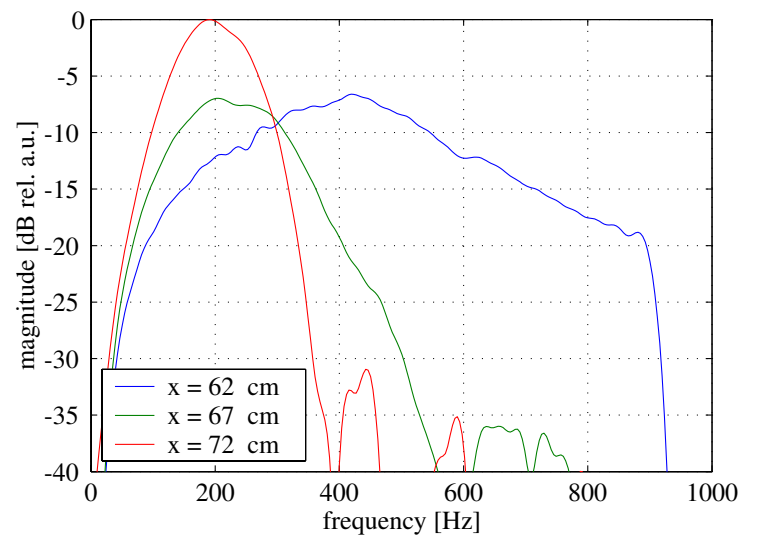

(a) Frequency spectrum for $\beta^{*}=0 / \mathrm{cm}$.

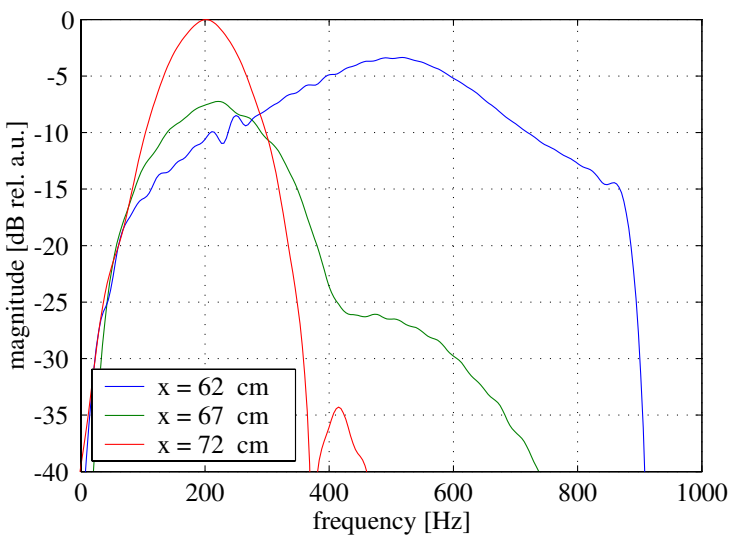

(b) Frequency spectrum for $\beta^{*}=0.63 / \mathrm{cm}$.



(c) RMS $\beta$ spectra (averaged $50-300 \mathrm{~Hz}$ )

Figure 6.11: Streamwise development of the actuator frequency and wavenumber spectrum, $U_{\infty}=18 \mathrm{~m} / \mathrm{s}$.

The side lobes are strongly suppressed (by $10 \mathrm{~dB}$ ) and the wavenumber distribution decreases by approximately $6 \mathrm{~dB}$ till the Nyquist wavenumber. This may not yet be sufficient to avoid aliasing effects in the error sensor signals.

If the spatial filtering reached by the design of the actuator is not sufficient, a second possibility to suppress high wavenumber components, may be to use the spatial filtering properties of the boundary layer itself. This may be achieved by increasing the streamwise distance between actuators and error sensors. 
This has been investigated by measuring the downstream development of the actuator spectrum for three different downstream distances $(\Delta x=1,6,11 \mathrm{~cm})$ in a similar manner. The result is shown in Fig. 6.11(a-c).

The figures $(a \& b)$ show the downstream development of the frequency spectra for the spanwise wavenumbers $\beta^{*}=0 \mathrm{~cm}^{-1}$ and $\beta^{*}=0.628 \mathrm{~cm}^{-1}$, latter being the Nyquist wavenumber of the sensor configuration. The shrinking of the spectra to the TS frequency range indicates that the downstream distance of $10 \mathrm{~cm}$ satisfies the far-field condition. Especially the harmonic part for $\beta^{*}=0.628 \mathrm{~cm}^{-1}$ is damped away slowly.

Fig. 6.11 (c) shows the streamwise development of the spanwise wavenumber spectra. It becomes obvious that apart from the streamwise amplification of the disturbances almost no change occurs concerning the shape of the spatial spectra. Therefore special care has to be taken on the initial wavenumber spectrum generated by the actuator.

In the case that the actuators used in the cancellation device do not produce a spatial spectrum of sufficient quality, an additional spatial filtering can be introduced by driving simultaneously the neighboring actuators. In the most simple way the tailoring of the driving signals can be realized by driving the neighboring actuators $K= \pm 1,= \pm 2, \cdots$ with the same signal but multiplied by a constant real factor $F_{p}^{K}$ and such forming a pseudo actuator ${ }^{2}$. This has been done for the Type II actuator for the weighting factors: $F_{p}^{0}=1, F_{p}^{ \pm 1}=0.7, F_{p}^{ \pm 2}=0.3$. The measured wavenumber spectrum shows a drop-off by about $15 \mathrm{~dB}$ up to the Nyquist wavenumber (see Fig. 6.10(d)) in comparison to $8 \mathrm{~dB}$ achieved by the single Type II actuator at the same Reynolds number (see Fig. 6.10 (c)). Thus this method of forming a pseudo actuator represents an option for fine-tuning the actuator wavenumber spectrum, which may be even improved further by a higher resolution of the actuators forming the pseudo actuator.

\footnotetext{
${ }^{2}$ This method may be refined by introducing any frequency depending filter matrix mapping the original driving signals (analytic signals) to the one of the pseudo actuator.
} 


\section{Chapter 7}

\section{Disturbance development in the boundary layer}

This chapter deals with the characterization of the boundary layer in which the experiments have been performed. In particular, the disturbance propagation within this boundary layer is of special interest with respect to the spatial wavenumber range of the propagating TS-waves.

\subsection{Blasius condition of the boundary layer}

The angle of incidence of the flat plate and the setting of the flap have been adjusted resulting in a pressure gradient close to zero in spanwise as well as in streamwise direction in order to obtain Blasius flow conditions.

Fig. 7.1(a) and (b) show the streamwise and spanwise variation of the pressure gradients $\frac{\partial c_{p}^{*}}{\partial x}$ and $\frac{\partial c_{p}^{*}}{\partial z}$. Herein $c_{p}^{*}$ denotes the pressure coefficient, given as

$$
c_{p}^{*}=\frac{p_{m}^{s}-p_{f}^{s}}{\frac{\rho}{2} U_{\infty}^{2}},
$$

where $p_{m}^{s}$ denotes the static pressure measured by a movable Prandl-tube. $p_{m}^{s}$ is compared by a sensitive pressure gauge to the detected static pressure $p_{f}^{s}$ of a Prandl-tube at a fixed streamwise position of $x=35 \mathrm{~cm}$. The moving probe was traversed outside the boundary layer in a distance of $4 \mathrm{~cm}$ from the wall.

As expected, the streamwise pressure gradient in Fig. 7.1 (a) is negative near the leading edge due to the accelerated flow. At $x=20 \mathrm{~cm}$, which is the position of the disturbance generator, some variations of the pressure gradient are observed. These 


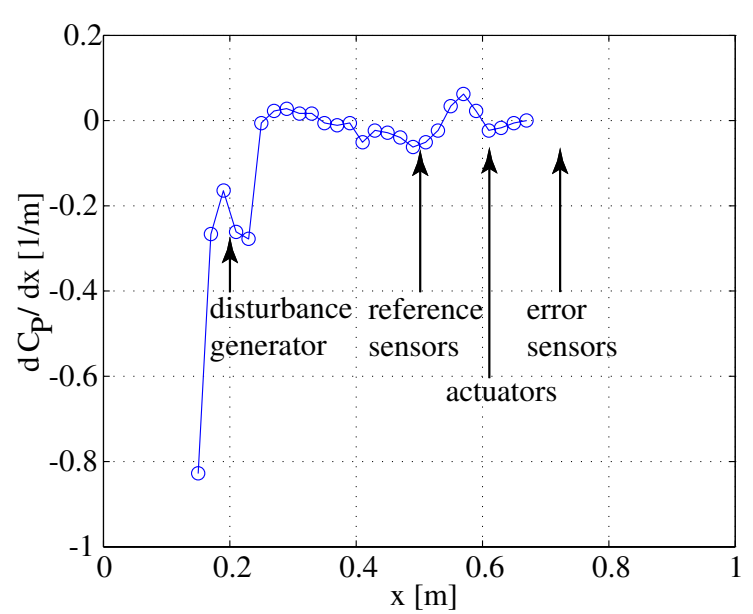

(a) Streamwise variation of $\frac{\partial c_{p}^{*}}{\partial x}$

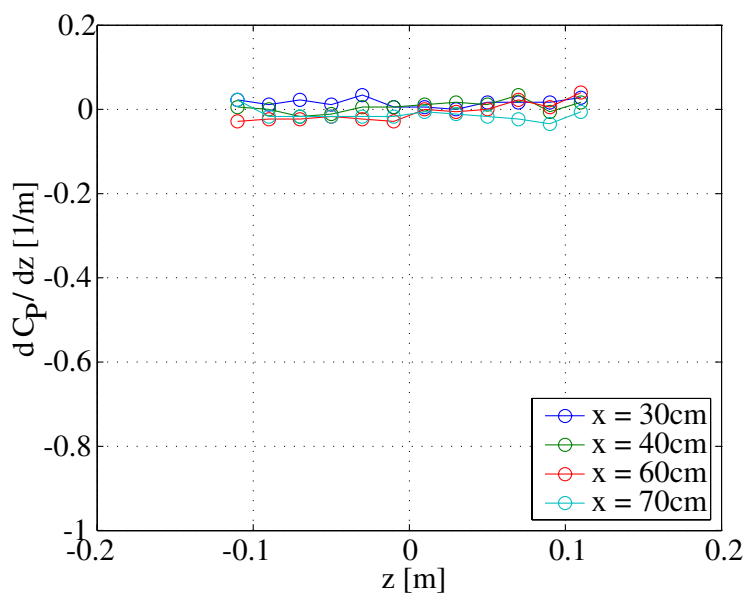

(b) Spanwise variation of $\frac{\partial c_{p}^{*}}{\partial z}$

Figure 7.1: Variation of the pressure gradient for $U_{\infty}=18 \mathrm{~m} / \mathrm{s}$.

are caused by the curvature of the gentle bump that has been generated by the $0.1 \mathrm{~mm}$ thick tape covering the actuator in order to reduce the acoustic effects mentioned in the previous chapter. The pressure gradient stays negative in this region therefore no separation of the boundary layer is expected. This has also been confirmed by boundary-layer profile measurements.

There are some variations of the pressure gradient visible in the region of the cancellation device. These may have been occurred due to changes of the surface curvature introduced by the implementation of the actuator box. Even though some deviations of the streamwise pressure gradient from zero have been observed, no obvious trend becomes visible. This applies also to the spanwise pressure gradient that is shown for some streamwise positions in Fig. 7.1(b).

Wall-normal profiles of the streamwise velocity component have been measured at various streamwise positions. These experiments have been carried out at the centerline of the $\operatorname{set}-u p(z=0 \mathrm{~cm})$. The obtained mean velocity profiles can be compared to the known Blasius solution in Fig. 7.2 ('theoretical'). Even though there is a visible deviation from the Blasius profile the agreement can be considered as good, taking into account the relatively large streamwise span of the measurements and the changes of the pressure distribution by the modifications of the plate (see Fig. 7.1). This is confirmed by the measured boundary layer parameters that are summarized for the different streamwise positions in table 7.1. Small deviations from the Blasius condition can be noticed by the slightly decreasing form-factor for streamwise positions greater 




(a) Streamwise mean velocity profiles in comparison to the Blasius solution.

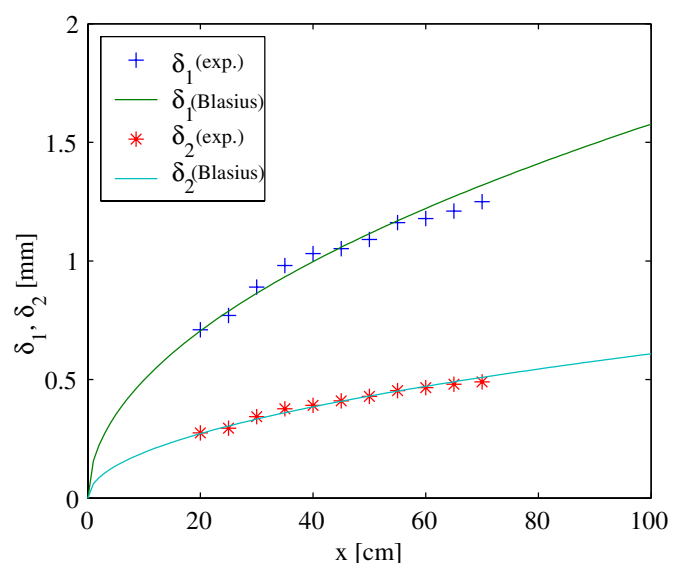

(b) The streamwise development of the experimentally obtained boundary layer thicknesses $\delta_{1}$ and $\delta_{2}$ compared to the Blasius solution.

Figure 7.2: Comparison of the experimental data to the Blasius solution.

than $x=45 \mathrm{~cm}$, indicating a slightly accelerated flow. However, the experimentally determined, averaged form-factor $H_{12}=2.57( \pm 0.04)$ agrees reasonably well with the one for Blasius-flow $\left(H_{12}=2.59\right)$. The experimentally obtained displacement thickness $\delta_{1}$ and the momentum thickness $\delta_{2}$ are plotted in Fig. $7.2(\mathrm{~b})$ versus the streamwise coordinate $x$. The Blasius solutions are plotted for comparison indicating also some deviations from the measured data. Because of these deviations no unique virtual leading edge could be determined which is often introduced to correct the Reynolds number considering the leading edge effect on the boundary layer.

Summarizing, the properties of the investigated boundary layer are close to the one of the Blasius solution.

Fig. 7.3 shows the stability diagram of Blasius flow. The curve of zero amplification separates the stable from the unstable region in the plane spanned by the nondimensional frequency $F$ and the Reynolds number Re based on the Blasius length $\delta$. Most experiments presented here have been carried out for a free-stream velocity of $U_{\infty}=18 \mathrm{~m} / \mathrm{s}$. Therefore the components of the wave cancellation device can be marked in the stability diagram. The theoretically expected frequency range of amplification is marked for each part of the device by the upper and lower bound of the curve of zero amplification.

It turns out that the measured disturbance spectra do not exactly reflect the ex- 


\begin{tabular}{||c|c|c|c|c|c|c||}
\hline $\mathrm{x}[\mathrm{cm}]$ & $\delta_{1}[\mathrm{~cm}]$ & $\delta_{2}[\mathrm{~cm}]$ & $H_{12}$ & $U_{e}[\mathrm{~m} / \mathrm{s}]$ & $R e_{\delta 1}$ & $R e$ \\
\hline \hline 20 & 0.71 & 0.28 & 2.58 & 18.53 & 871 & 488 \\
\hline 25 & 0.77 & 0.29 & 2.61 & 18.15 & 925 & 546 \\
\hline 30 & 0.89 & 0.34 & 2.59 & 17.75 & 1045 & 598 \\
\hline 35 & 0.98 & 0.38 & 2.60 & 18.09 & 1174 & 646 \\
\hline 40 & 1.03 & 0.39 & 2.64 & 17.86 & 1219 & 690 \\
\hline 45 & 1.05 & 0.41 & 2.57 & 18.07 & 1257 & 732 \\
\hline 50 & 1.09 & 0.43 & 2.54 & 18.23 & 1315 & 772 \\
\hline 55 & 1.16 & 0.45 & 2.56 & 18.14 & 1394 & 809 \\
\hline 60 & 1.18 & 0.47 & 2.53 & 17.96 & 1401 & 845 \\
\hline 65 & 1.21 & 0.48 & 2.52 & 18.25 & 1462 & 880 \\
\hline 70 & 1.25 & 0.49 & 2.55 & 17.73 & 1467 & 913 \\
\hline
\end{tabular}

Table 7.1: Boundary layer parameters: $\delta_{1}, \delta_{2}, H_{12}, U_{e}, R e_{\delta 1}$ and $R e$

pected frequency range, depending on the disturbance excitation. In general, however, the measured disturbance spectra are integral quantities of the excitation spectrum subjected to the dispersion relation while propagating downstream. This may be approximated by the superposition of frequency wavenumber components

$$
\mathrm{d} \tilde{u}(x, y, t)=\mathrm{d} \tilde{u}_{0}(y, \omega, \beta, R e(x)) e^{i\left(\int_{\hat{x}}^{x} \alpha\left(\omega, \beta, R e\left(x^{\prime}\right)\right) d x^{\prime}+\beta z-\omega t\right)},
$$

where $\alpha, \beta$ denote the streamwise and spanwise wavenumber and $\omega$ the circular frequency. Herein the dispersion relation $\alpha(\omega, \beta, R e)$ describes the dynamics of the boundary layer. Therefore the measured spectra depend on the excitation spectra and the streamwise location where the disturbances have been introduced into the boundary layer. This may explain the deviations in the presented spectra. Especially in the case of 'natural' TS-waves the excitation process and the initial frequency spectrum are unknown. The observed shift to higher frequencies can be explained by the large streamwise distance between the points of the large receptivity and the streamwise position where the measurements have been performed. 


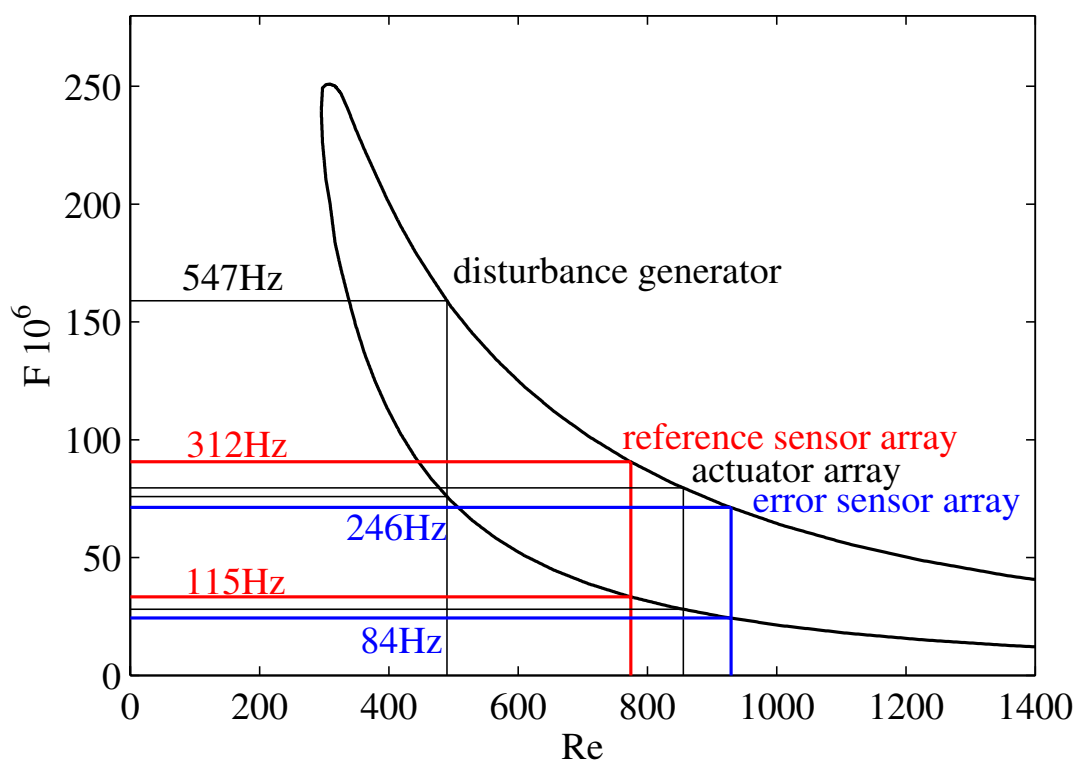

Figure 7.3: The stability diagram for 2D disturbances in Blasius flow, the device components are marked for $U_{\infty}=18 \mathrm{~m} / \mathrm{s}$

\subsection{Point source experiment}

Concerning the spatial development of wave-packets, first experimental investigations of good quality signal-to-noise ratio have been carried out by Gaster and Grant (1975). They excited a point-source by a series of short duration acoustic pulses and averaged the recorded hot-wire signal traces in a phase-locked manner. The hot-wire was traversed in spanwise direction at several streamwise locations. The obtained results have been compared to linear stability theory by Gaster (1975) and later to a direct numerical simulation adapted to the conditions of Gaster's experiment, (Konzelmann 1990).

In contrast to the aforementioned investigations, the present point source experiments on the dispersion relation of the actual boundary layer have been carried out considering linearly correlated signal components only. In these tests, a continuous white noise signal generated the suction and blowing through a narrow slit, $3 \mathrm{~mm}$ long in spanwise direction, and in such a way served as a continuously driven point source. The excited velocity fluctuations were measured by a hot-wire probe at various lateral and streamwise positions, starting above the disturbance generator and ending at the error sensor position, in $5 \mathrm{~cm}$ steps. The transfer functions to the positions of the hot-wire were measured by means of the system identification (see section 4.2.1).

These measurements have been carried out at the same free-stream velocity of 
$18 \mathrm{~m} / \mathrm{s}$ that has been preferably used in the active wave cancellation experiments described below. Therefore, also natural TS-waves have been present in the boundary layer generating maximum RMS-values of $0.6 \%$ of the free-stream velocity. The difficulty was to choose an appropriate excitation amplitude, preferably as small as possible in order not to change the boundary layer characteristics by nonlinear effects but large enough to obtain a proper signal-to-noise ratio.

In order to obtain the maximum achievable signal amplitude of the excited disturbances, measurements of the wall-normal profile of streamwise velocity fluctuations to determine the location of the TS maximum have been made before the main experiments. The measurements have been performed at each streamwise position at the centerline of the set-up $(z=0 \mathrm{~cm})$.

In contrast to the experiments by Gaster and Grant (1975) who preferred the flat outer maximum, the present measurements of the spanwise profiles have been made at the inner maximum of the wall-normal RMS-profile.

The flat outer maximum is a good choice to minimize errors caused by a drift of the wall distance while traversing. Due to the differing wall-normal TS-eigenfunctions for 2D- and 3D waves, the outer maximum will also lead to an underestimation of 3D components whereas the sharp inner maximum has got a higher amplitude than the outer maximum and therefore improves the SNR. With regard to 3D components, problems can occur due to the shift of the inner maximum away from the wall for increasing inclination angle of the 3D TS-wave. The theoretically expected wall-normal profile of the TS-amplitude is shown in Fig. 7.9 (b) for three different spanwise wavenumbers.

The streamwise development of the measured velocity fluctuations with and without excitation is illustrated by Fig. 7.4 (a-l). Besides the mean velocity profiles the RMSprofiles are plotted for the case with point-source excitation (marked by 'with PS') and for the case of natural TS-waves. The RMS-values are determined in the frequency range $60-1000 \mathrm{~Hz}$ and are normalized by the maximum local velocity $U_{e}$ at the boundary layer edge.

The RMS-profile ('with PS') in Fig. 7.4(a) is determined directly above the point source and therefore is a measure of the excitation amplitude. Within a downstream distance of about $15 \mathrm{~cm}$ from the point-source the formation of a typical TSprofile containing the outer maximum can be realized. This is caused by the dispersion related amplification of parts of the excitation spectrum. Also explained by this damping is the decreasing difference between the RMS-profiles of both cases.

With increasing downstream distance from the excitation source ('with PS') the velocity fluctuations grow, leading to nonlinear effects in the rear part of the measurement region. These are first indicated by the deformation of the signal time traces for $x \geq 50 \mathrm{~cm}$. At the far end of the measurement region $(x>60 \mathrm{~cm})$ the RMS-values grow over $>1 \%$ which is related to the occurrence of 'spikes' in the observed time traces. 
Singular 'spikes' occurred already at $x>55 \mathrm{~cm}$. Due to these nonlinear effects, not all of the results obtained in this region by the point source excitation may be applied directly to the case of naturally occurring TS-waves.

TS-waves generated by the excitation of the point-source disturbance generator with white noise have both a broad frequency spectrum and a broad wavenumber spectrum. In order to investigate the transfer properties of the boundary layer and thereby its dispersion relation the spatial disturbance evolution has been studied by means of transfer functions.

For each of the selected x-positions a series of 41 impulse responses corresponding to the transfer functions between the driving signal and the signal of the movable hotwire sensor has been measured, each at a different spanwise coordinate. So a lateral span of $20 \mathrm{~cm}$ was covered, centered around the location of the point source at $z=0 \mathrm{~cm}$. The spanwise series of impulse responses comprises the times which are needed by the various components of the pulse to propagate from the point-source where the pulse is excited to each of the spanwise positions at the streamwise location where it is detected by the hot-wire sensor.

The impulse responses have been determined by means of adaptive filters. The system identification scheme has been applied in the two configurations described in chapter 4 . However, the data, obtained by the configuration which takes into account the influence of the generation and the measurement process, turned out to be impaired by an echo in the estimate of transfer function $H_{P S}(z) H_{H W}(z)$. Therefore, the results of the standard system identification are presented here (see section 7.5).

The filter $W_{1}(z)$ has been adapted directly between the white noise signal sent to the actuator and the measured hot-wire signal. Besides the physical transfer function $P(z)$ the adapted filter contains also the transfer properties of the generation process in this case, namely the 'point-source' disturbance generator $H_{P S}(z)$, as well as the transfer function of the measuring act including the hot-wire sensor, the lowpass filter, cables etc., named by $H_{H W}(z)$. Hence, the adapted transfer function is given by

$$
W_{1}(z)=H_{P S}(z) P(z) H_{H W}(z)
$$

The sFTF-algorithm has been used for the adaptation of the filters because of its property that the time of convergence is independent of the input-signal statistics. The exponential time window was chosen large $(\lambda=0.9999995)$ to obtain reliable averages. If the adaptation error, defined via the change in filter taps, was below a certain limit for a number of iterations, the program saved the adapted filter taps, sent a trigger-signal to the traversing mechanism and the procedure was repeated at the next spanwise position. 


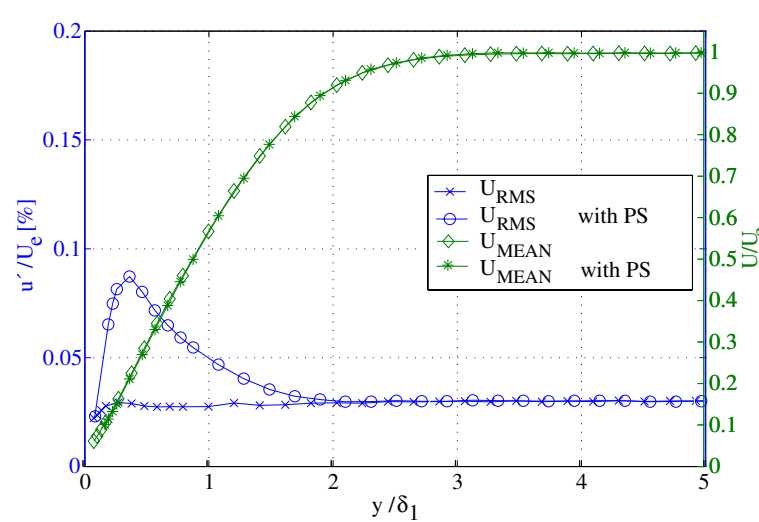

(a) $x=20 \mathrm{~cm}$

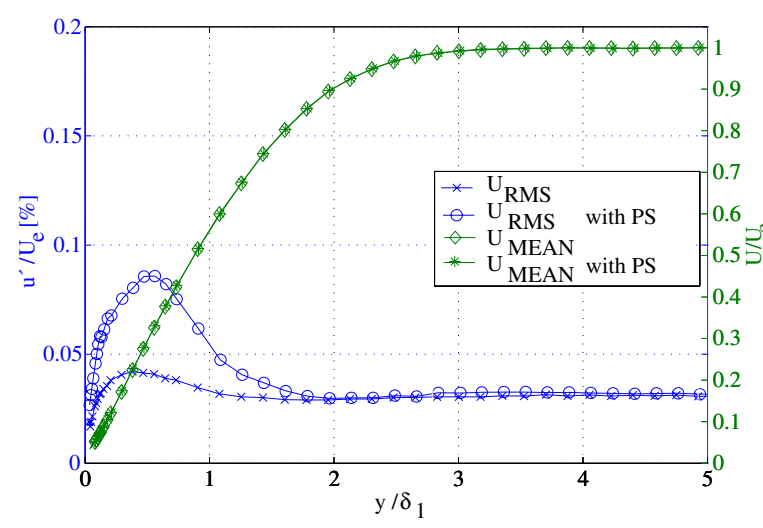

(c) $x=30 \mathrm{~cm}$

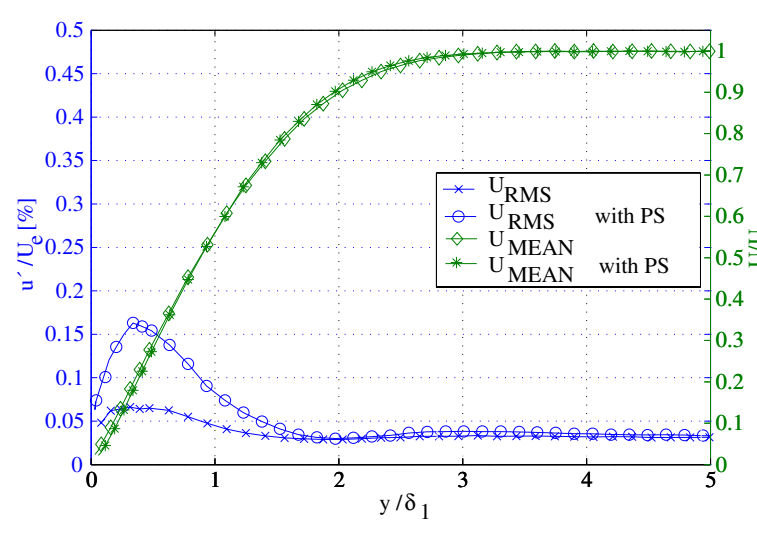

(e) $x=40 \mathrm{~cm}$

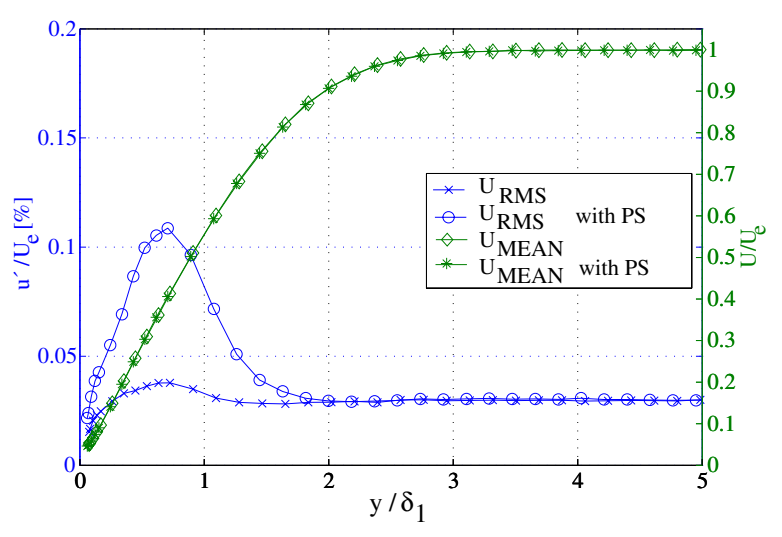

(b) $x=25 \mathrm{~cm}$

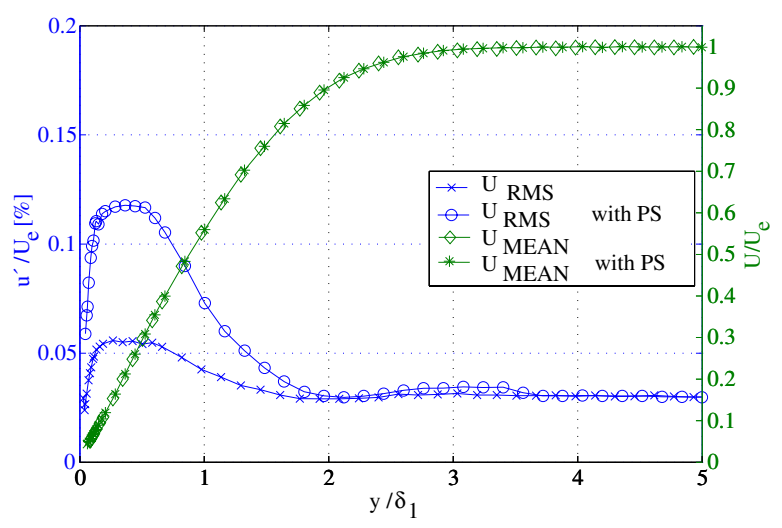

(d) $x=35 \mathrm{~cm}$

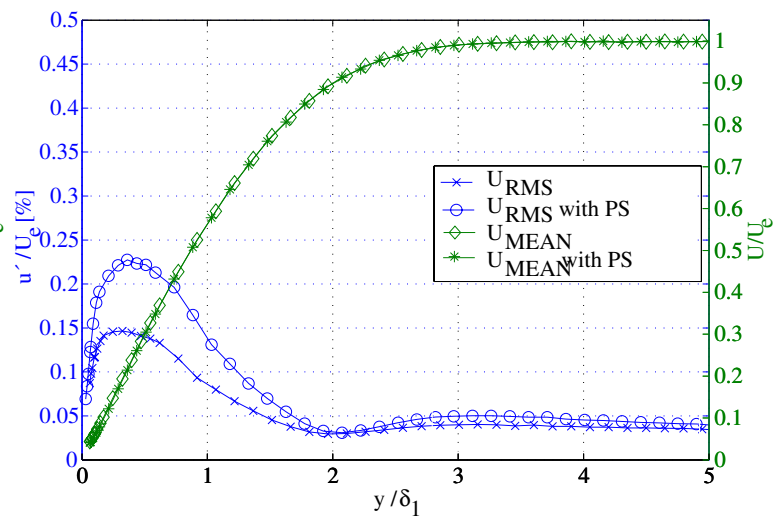

(f) $x=45 \mathrm{~cm}$ 


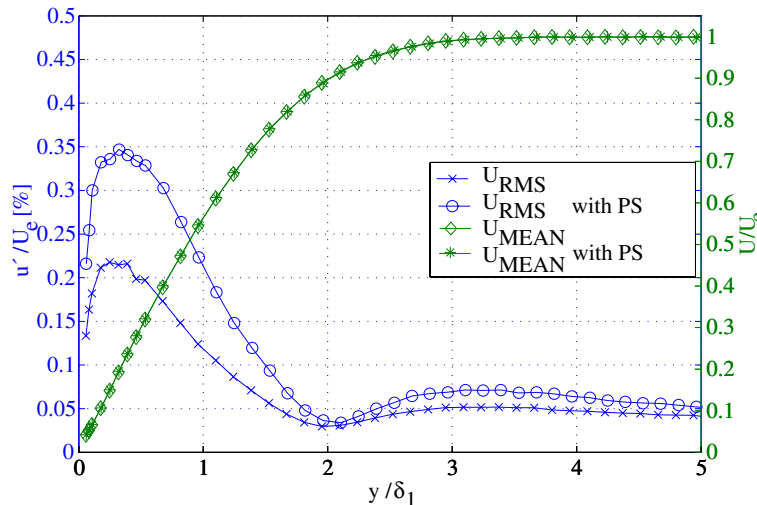

(g) $x=50 \mathrm{~cm}$

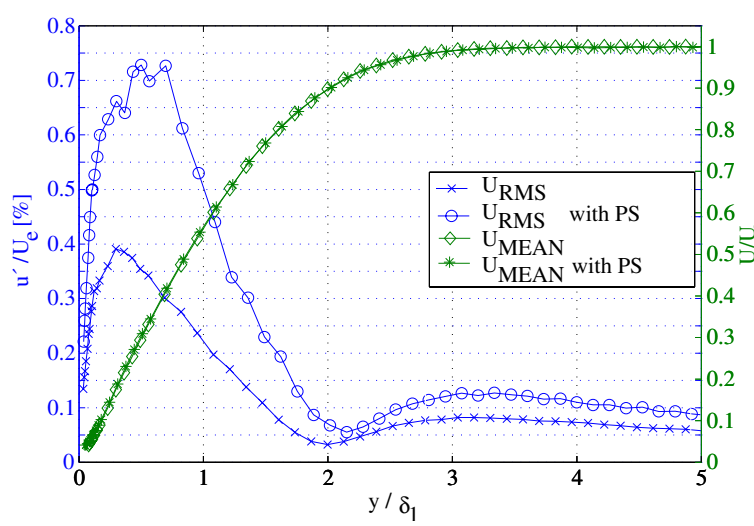

(i) $x=60 \mathrm{~cm}$



(k) $x=70 \mathrm{~cm}$

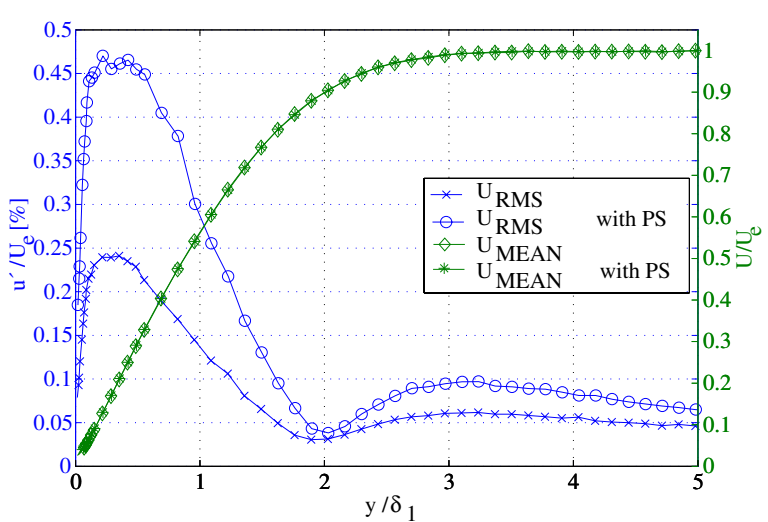

(h) $x=55 \mathrm{~cm}$

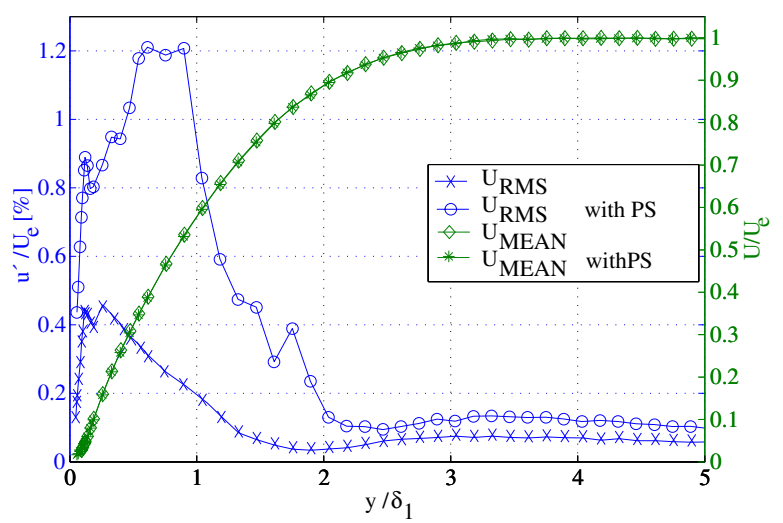

(j) $x=65 \mathrm{~cm}$

Figure 7.4: Streamwise development of the mean velocity profiles and the RMS-profiles for natural TS-waves and for the excitation by the point source (PS). The profiles are measured at the centerline $(z=0)$ and the wall normal coordinate is normalized by $\delta_{1}$. Note that the scales of $u^{\prime} / U_{\infty}$ differ between the diagrams for the different x-locations. 


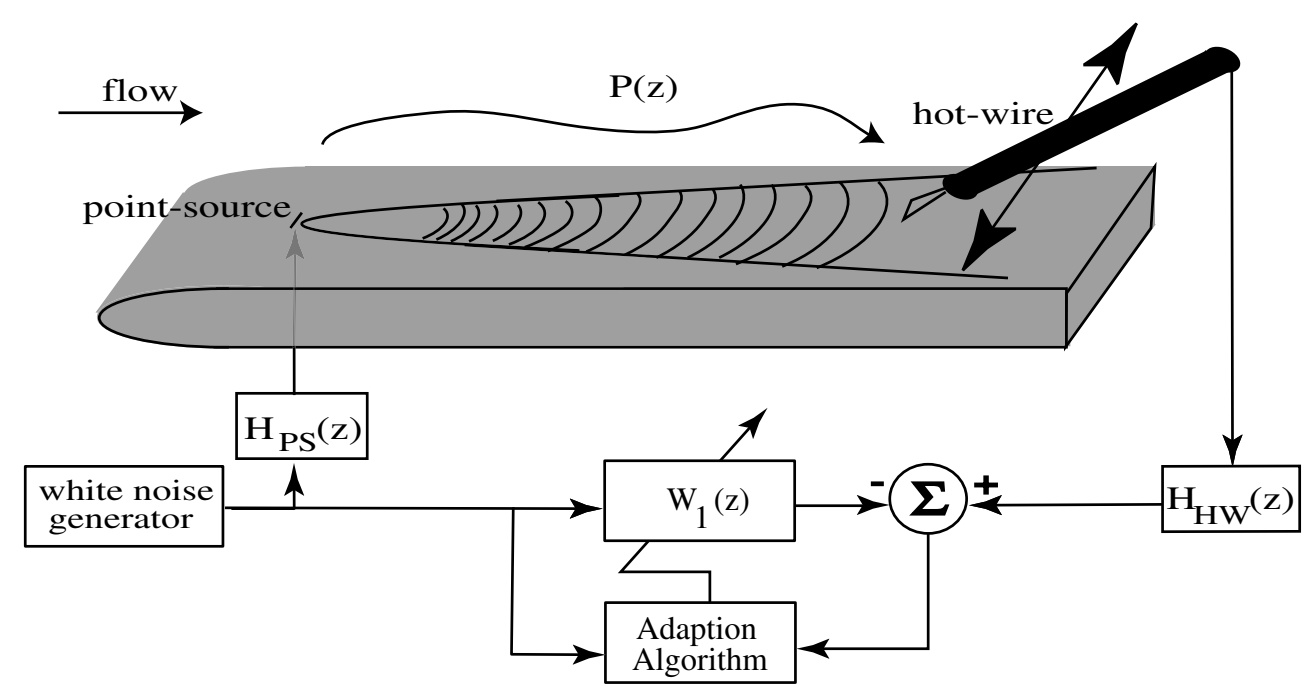

Figure 7.5: Set-up and system identification scheme in the point-source experiment.

In Fig. 7.6 the obtained impulse responses of $W_{1}(z)$ are lined-up for each streamwise position, thus forming a series of spanwise pulses that are separated by black lines. The amplitude of each pulse representing the linearly correlated part of the wave-packet is normalized to one, due to the large difference in amplitudes $(\approx 30 \mathrm{~dB})$. The point at $x=20 \mathrm{~cm}$ indicates the pulse directly above the source. By the increasing downstream distance between the point source and the hot-wire the propagation time of the pulse increases, too. The band-pass characteristics of TS-instability in frequency and wavenumber domain leads to the spreading of the pulse in time and spanwise coordinate. The smoothly contoured wave packet, which reaches its peak amplitude close to the center, is gradually distorted and far downstream two amplitude maxima are formed on either side of the center line.

Downstream of $x=55 \mathrm{~cm}$ nonlinear effects occur deforming the pulse. The pulses deviate from the bow-shape measured for smaller distances to the source, forming a structure that looks like footprints of lambda vortices. This assumption is supported by the observed 'spikes' in the signal traces and the strong sub-harmonic part of the monitored frequency spectra. ${ }^{1}$ In this context, it is remarkable that only linear correlations between the excitation signal and the hot-wire signal have been evaluated. This means that if these patterns originate from lambda vortices, they seem to be determined already by the excitation signal in a reproducible way.

However, in order to obtain a measure for the streamwise influence of the dispersion,

\footnotetext{
${ }^{1}$ Gaster made similar observations which have been confirmed by the DNS of Konzelmann.
} 
7.2 Point source experiment

79

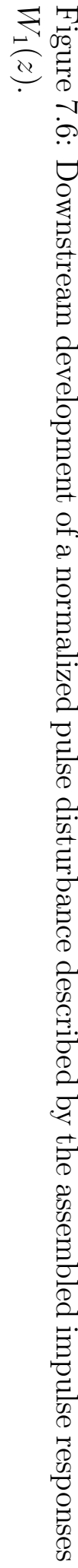




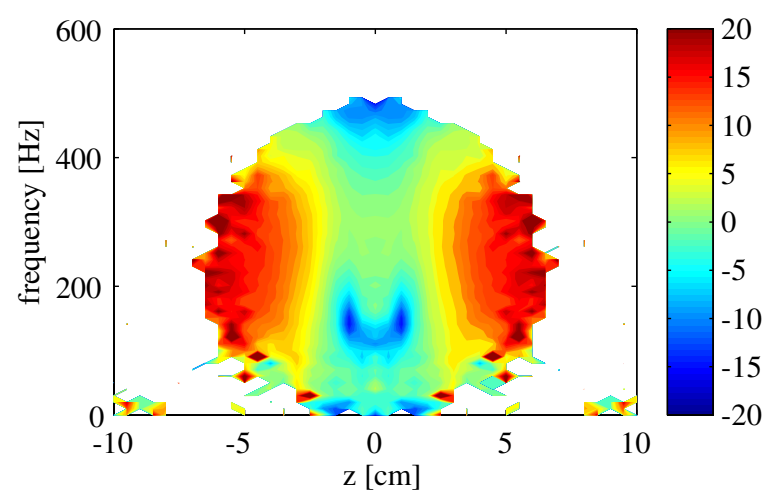

(a) $x=35-40 \mathrm{~cm}$



(c) $x=50-55 \mathrm{~cm}$

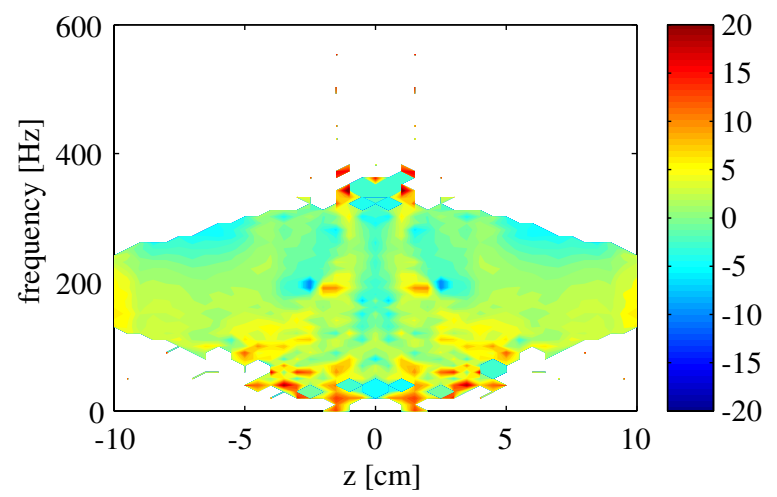

(e) $x=65-70 \mathrm{~cm}$

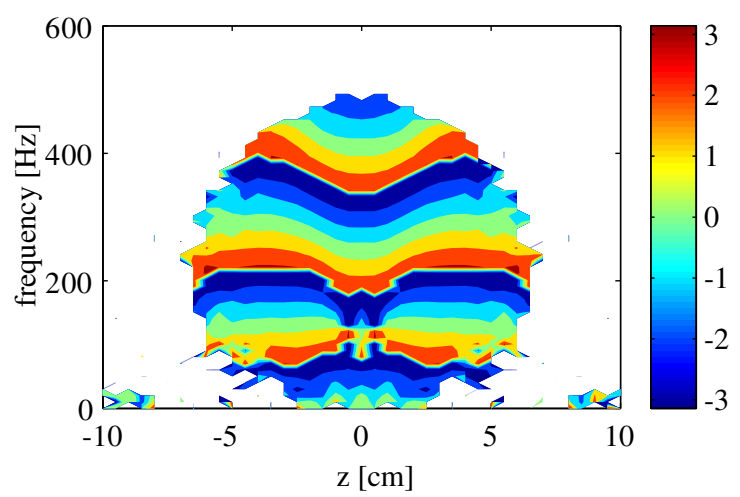

(b) $x=35-40 \mathrm{~cm}$

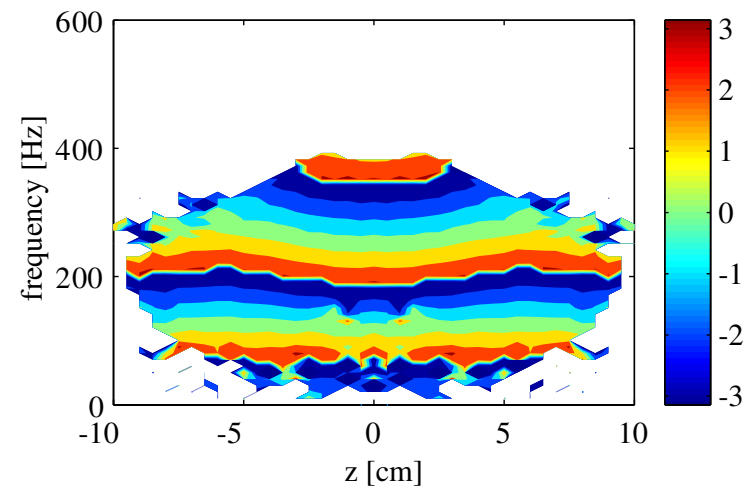

(d) $x=50-55 \mathrm{~cm}$

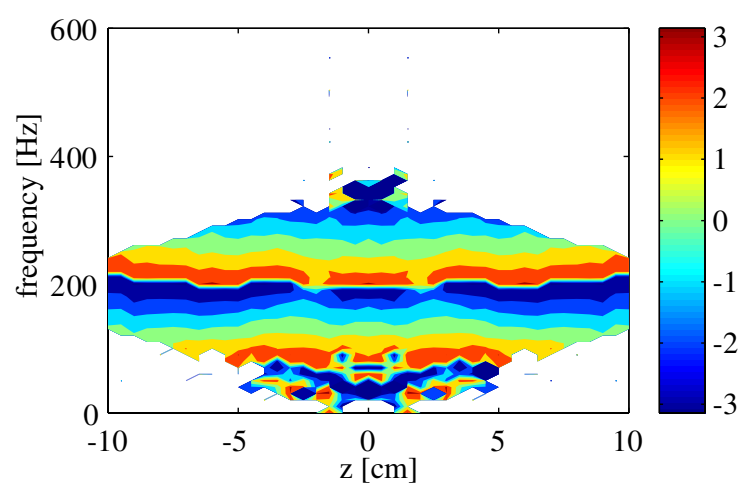

(f) $x=65-70 \mathrm{~cm}$

Figure 7.7: Spanwise magnitude (left) and phase (right) distribution of the transfer function between the indicated streamwise locations. 
the transfer properties of the boundary layer have been determined by calculating the flow transfer function $H^{12}(f)^{2}$ from one streamwise position $X_{1}$ to the following denoted by $X_{2}$. This can be achieved by dividing the transfer functions from the source to both considered positions. Hence, the transfer function $H^{12}(f)$ describing the propagation from $X_{1}$ to $X_{2}$ is given by

$$
H^{12}(f)=\frac{H_{X_{2}}(f)}{H_{X_{1}}(f)}
$$

It is reasonable here to consider only that part of the Fourier transforms which is less than $50 \mathrm{~dB}$ below the maximum component. The results are shown for three different streamwise positions in Fig. 7.7. On the left, the figure shows the spanwise distribution of the magnitude (color scale in $\mathrm{dB}$ ) and, on the right the spanwise variation of the phase is shown. The amplification at the spanwise edge of the pulse decreases with increasing downstream coordinate indicating a lower lateral pulse spreading. Also the variation in the spanwise phase distribution nearly disappears.

The frequency dependent phase velocity $c_{p h}(f)$ and group velocity $c_{g r}(f)$ of the pulse have been determined for the center position $(z=0)$. Theses have been calculated from the phase response $\arg \left\{H^{12}(f)\right\}$ via the associated phase delay

$$
\tau_{p h}(f)=-\frac{1}{2 \pi} \frac{1}{f}\left(\arg \left\{H^{12}(f)\right\}\right) .
$$

and the group delay

$$
\tau_{g r}(f)=-\frac{1}{2 \pi} \frac{\mathrm{d}}{\mathrm{d} f}\left(\arg \left\{H^{12}(f)\right\}\right)
$$

This has been evaluated for the center transfer functions in streamwise range $20 \mathrm{~cm} \leq$ $x \leq 50 \mathrm{~cm}$. The averaged result divided by the free-stream velocity is shown in Fig. 7.8. This agrees well with the one determined for 2D disturbances (e.g. in (Evert 2000)) except for the low frequencies, where even a crossing of both lines occurs. The error bars in this region indicate a high uncertainty. For comparison the envelope velocity has been determined from the time delay of the maximum of the pulse envelopes to $c_{e}=40.6 \%$ of the free-stream velocity $U_{\infty}$. This corresponds approximately to the group velocity at $200 \mathrm{~Hz}$.

In the following the streamwise development of the pulses $\left(W_{1}(z)\right)$ has been evaluated in the frequency-wavenumber domain leading to the wavenumber spectra shown Fig. $7.10(\mathrm{a}-\mathrm{k})$. Within a distance of about $15 \mathrm{~cm}$ downstream of the point-source the high-frequency and high-wavenumber components are damped away significantly, so that the disturbance spectrum is approximately confined to the range expected by linear stability analysis Fig. 7.9 (a). The Reynolds numbers pertaining to the downstream coordinate are given in the legend in Fig. 7.10.

\footnotetext{
${ }^{2}$ The variable is changed to the frequency $f$ based on the previously explained capability of digital filters to model continuous transfer functions.
} 


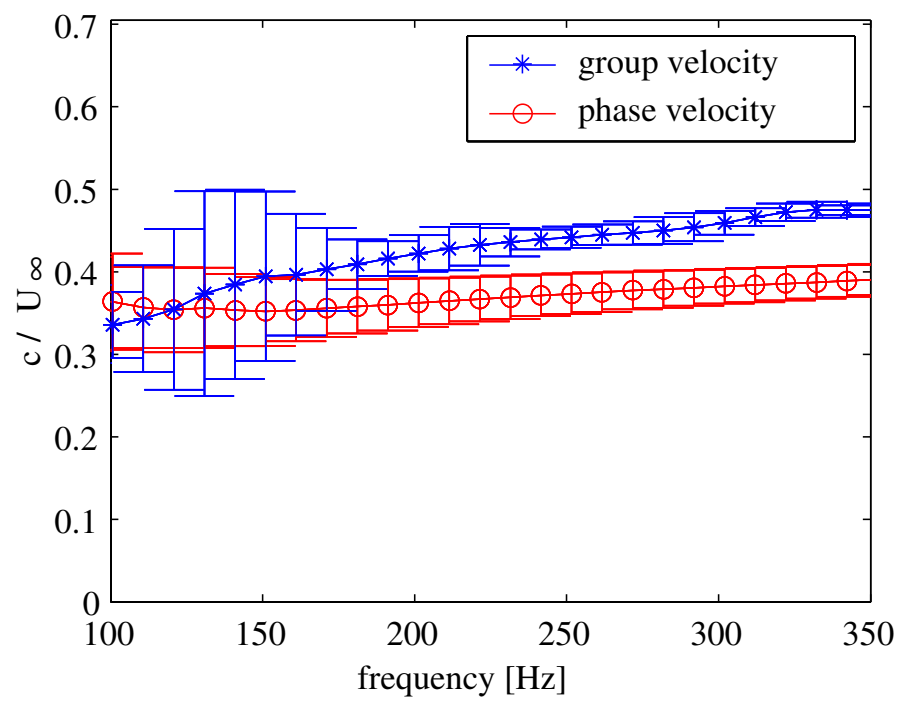

Figure 7.8: Phase and group velocity versus frequency. The error bars denote the standard deviation of the average calculated for the region $20 \mathrm{~cm} \leq x \leq 50 \mathrm{~cm}$.



(a) Stability diagram for different spanwise wavenumbers (PS: point-source, ES: error sensor).



(b) Eigenfunctions of the least stable mode for three different spanwise wavenumbers.

Figure 7.9: Spatial stability characteristics of the investigated boundary layer. 
The above mentioned nonlinear effects also become visible in the spectra by higher wavenumber components that are occurring outside the contiguous part of the spectrum as indicated in Fig. 7.10 by the arrow marked by the number '1'. The high wavenumber components turn up in the same frequency range as the fundamental part but at approximately twice the highest wavenumber component found in the fundamental part. Decreasing the excitation amplitude leads to the disappearance of these high wavenumber components as indicated by the RMS spectra, shown in Fig. 7.11, resulting from varying excitation amplitudes $(a)$. These nonlinear effects become visible for streamwise coordinates larger than $x=55 \mathrm{~cm}$.

Remarkable is again that these high wavenumber components are linearly correlated to the excitation signal according to the applied data evaluation method. Therefore they must have been generated phase-locked to the disturbances in the main part of the spectrum. Small portions of these components are also visible in the spectra evaluated by Gaster and later by Konzelmann. The difference between the present result and their result may be explained by the different wall-normal locations, i.e. by the disappearance of the outer amplitude maximum in the eigenfunction of the high spanwise wavenumber components.

The arrow labelled by the number ' 2 ' in Fig. 7.10 points to the dent in the contiguous part of the spectrum at low frequencies $(80-180 \mathrm{~Hz})$ and small spanwise wavenumbers $\left(-1.3\right.$ to $\left.+1.3 \mathrm{~cm}^{-1}\right)$. This can be explained by the occurrence of subharmonic components that have been observed in the monitored spectra but are not considered by the evaluation method used in the present study which considers linear correlations, only. In contrast, the data from Gaster and Konzelmann, which contain all measured velocity fluctuations, i.e. also nonlinearly generated second order components, show a subharmonic peak at this location of the frequency-wavenumber plane.

In general the agreement between the present results and the previously mentioned studies is good, taking into account the different experimental conditions and excitation amplitudes. This is confirmed by the qualitative comparison of the pulse with a result obtained by Konzelmanns DNS in Fig. 7.12 (a) \& (b). These pulses are both obtained in the region of the inner pulse maximum whereas Fig. 7.12 (c) shows the pulse at the outer maximum, also obtained by Konzelmann who adjusted the conditions to Gasters experiment. The agreement of the pulses at the inner maximum is surprisingly good considering the different sizes of the source and the unknown excitation amplitudes.

Coming back to the actual aim of this investigation, namely to obtain the dispersion characteristics the active wave cancellation system is exposed to, one may neglect the occurrence of these nonlinear effects. Doing so and taking into account the stability diagram from Fig. 7.9 (a), it can be concluded that in the region of the cancellation device disturbances are amplified in the spanwise wavenumber range of $-2 \mathrm{~cm}^{-1}<$ $\beta<2 \mathrm{~cm}^{-1}$ for linear propagation. In retrospect, the excitation amplitude should 


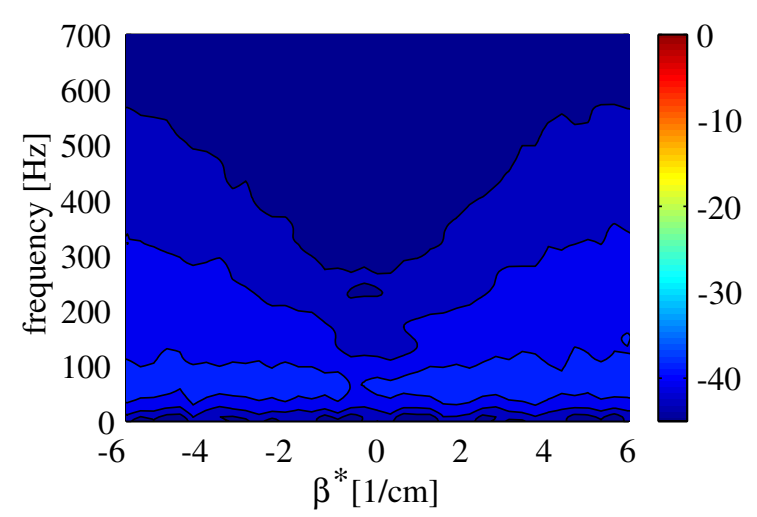

(a) $x=20 \mathrm{~cm}, R e=488$

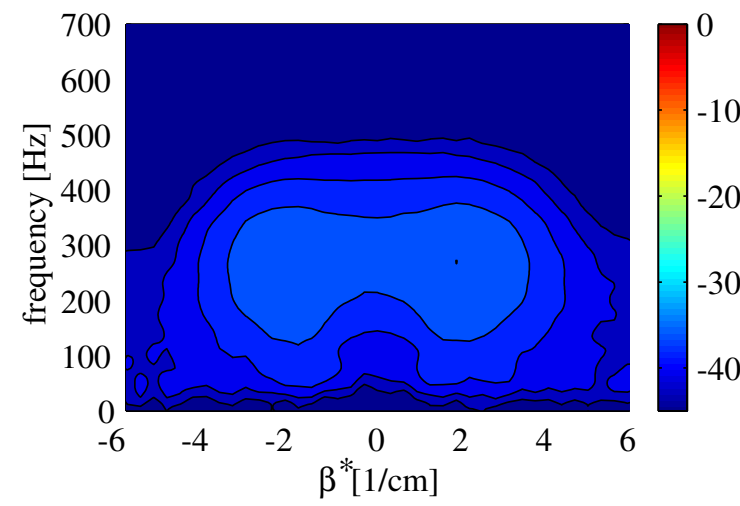

(c) $x=30 \mathrm{~cm}, R e=598$

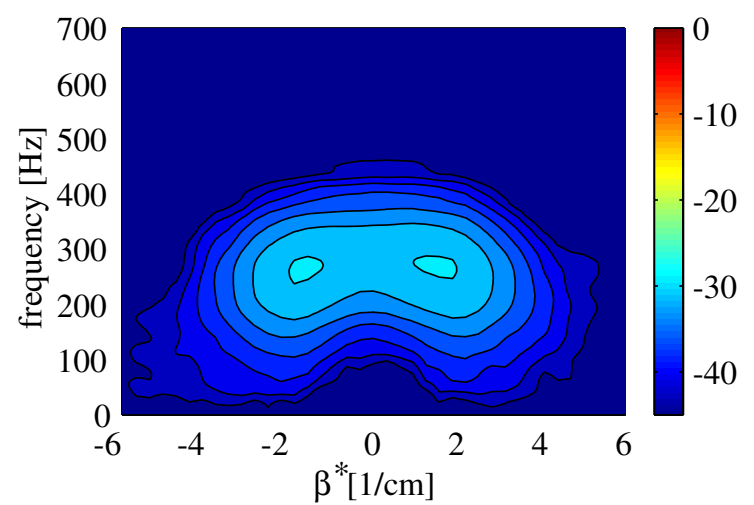

(e) $x=40 \mathrm{~cm}, R e=690$

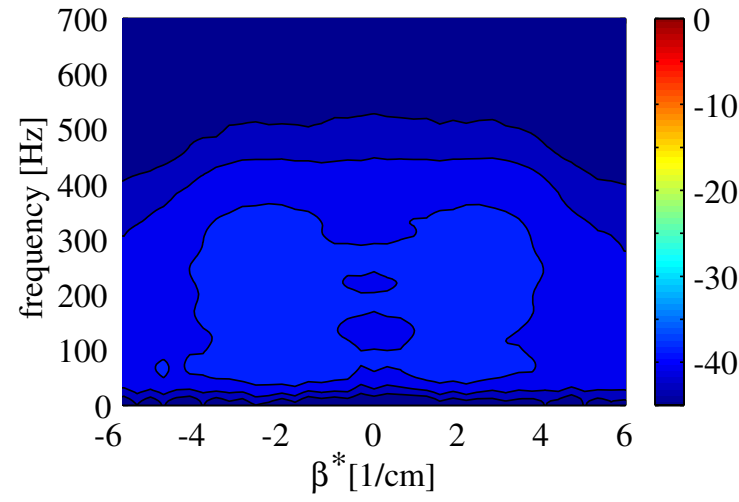

(b) $x=25 \mathrm{~cm}, R e=546$

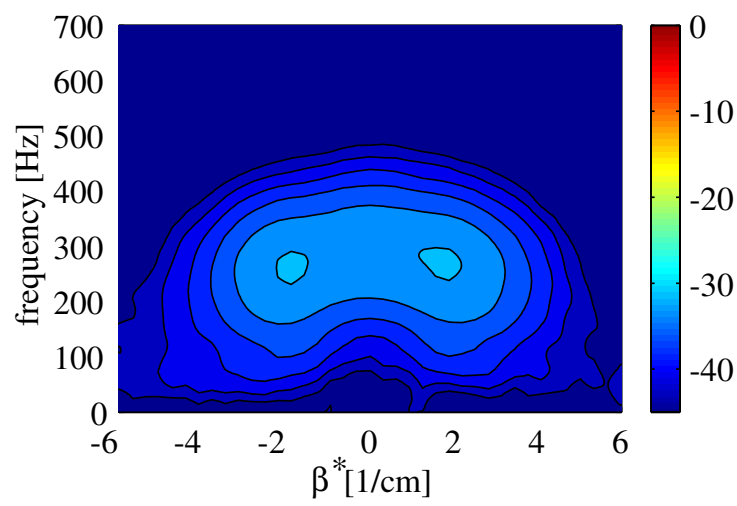

(d) $x=35 \mathrm{~cm}, R e=646$

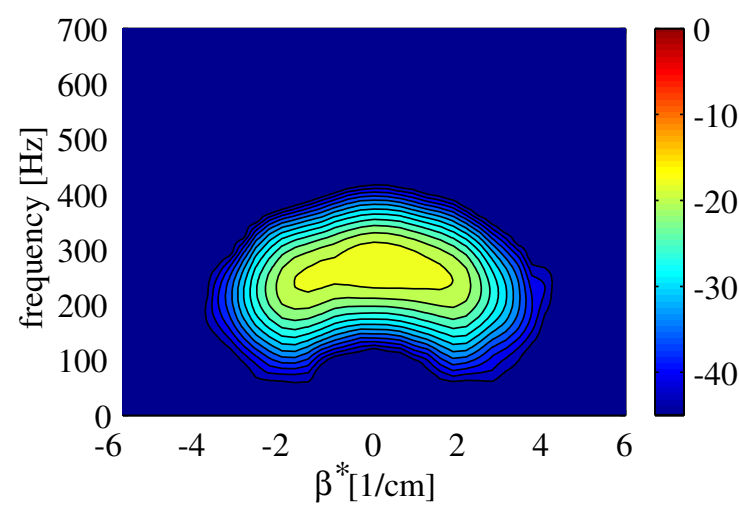

(f) $x=45 \mathrm{~cm}, R e=732$ 


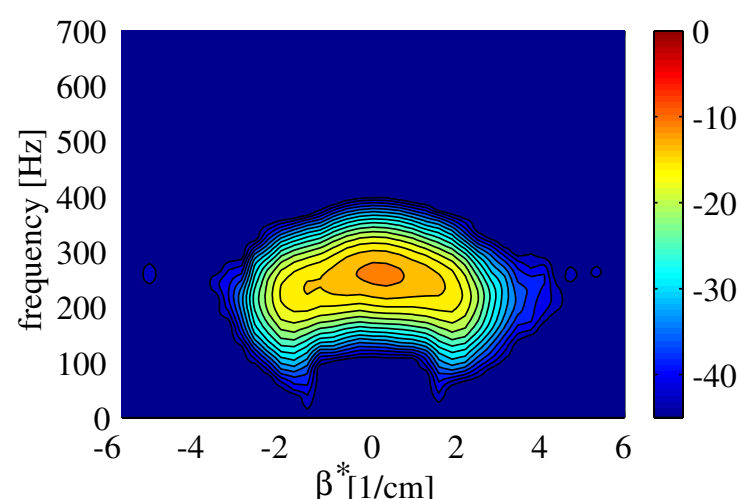

(g) $x=50 \mathrm{~cm}, R e=772$

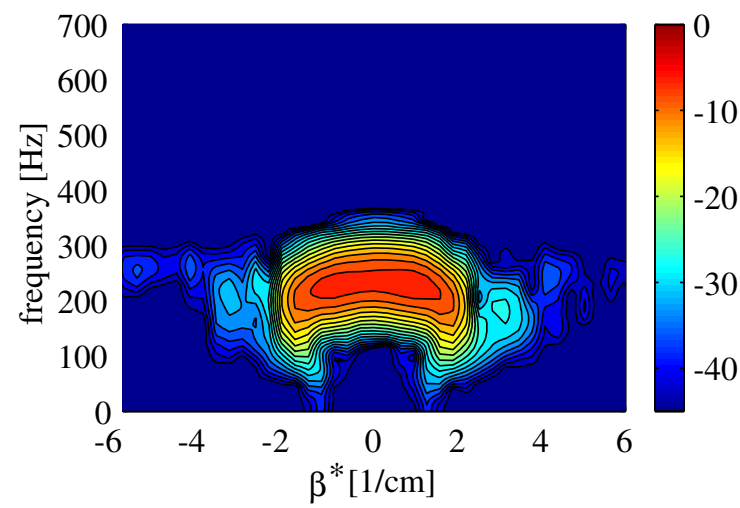

(i) $x=60 \mathrm{~cm}, R e=845$

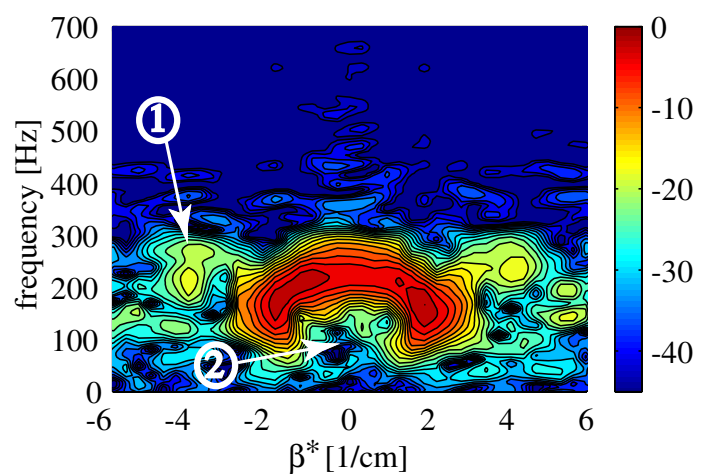

(k) $x=70 \mathrm{~cm}, R e=913$

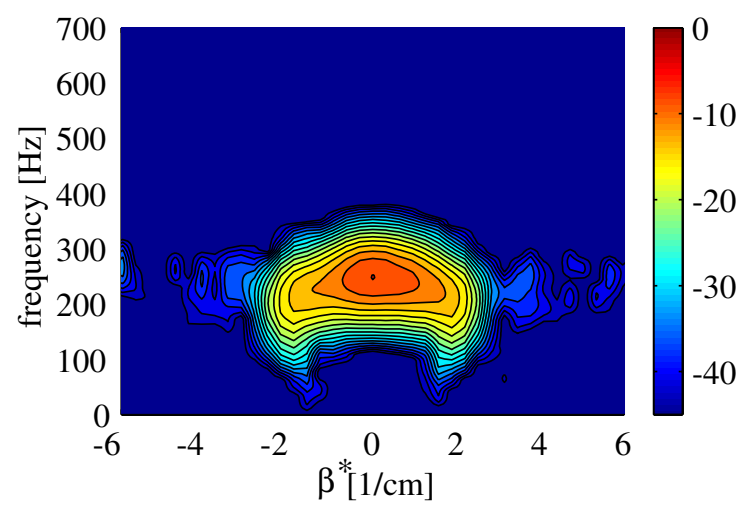

(h) $x=55 \mathrm{~cm}, R e=809$

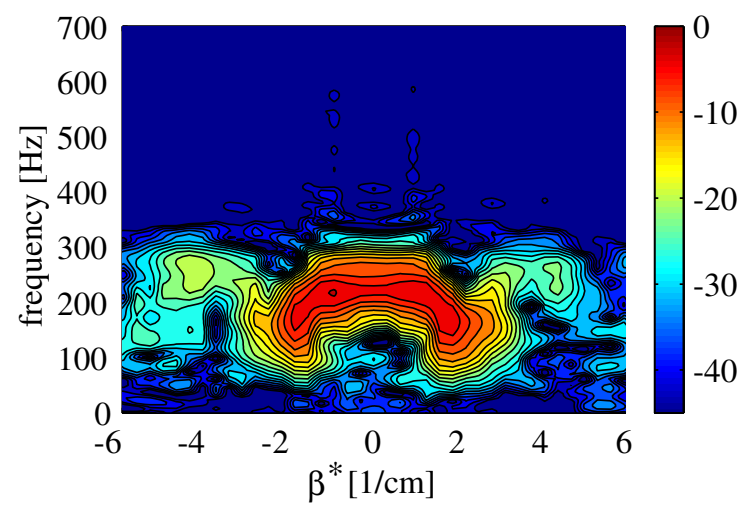

(j) $x=65 \mathrm{~cm}, R e=880$

Figure 7.10: Downstream development of a point-source disturbance pulse $\left(W_{1}(z)\right)$ in the frequency-wavenumber domain (for details see table 7.1). 


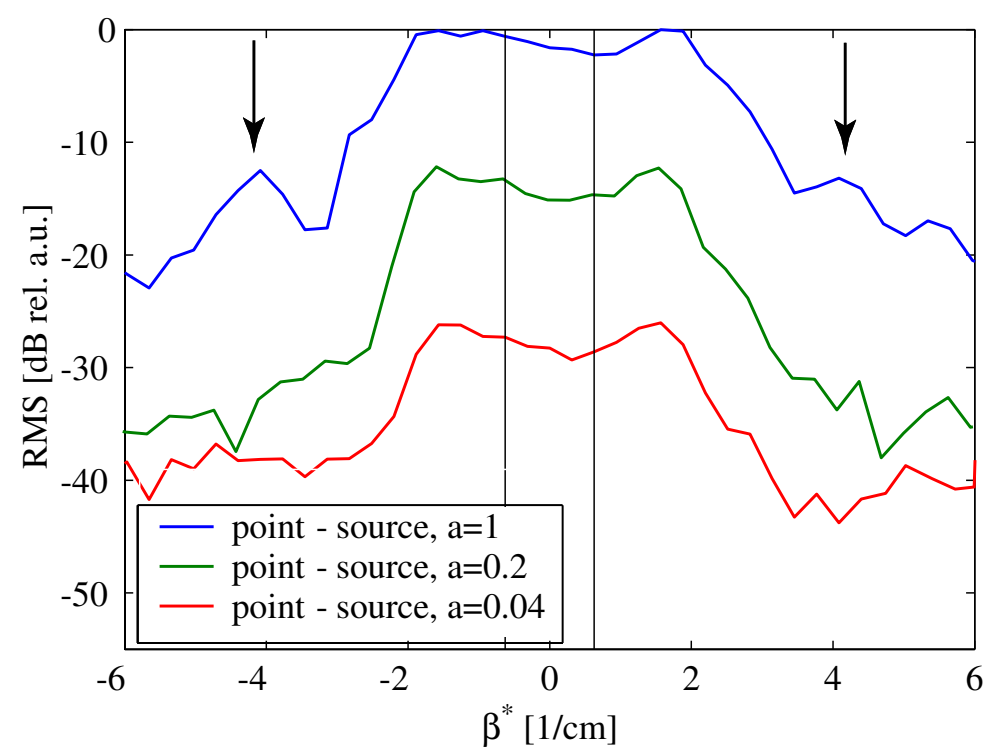

Figure 7.11: RMS-spectra of the point-source disturbance for different excitation amplitudes, at $x=72 \mathrm{~cm}$.

have been chosen at least $15 \mathrm{~dB}$ lower to achieve the aimed comparability to the wave cancellation experiments described below. Nevertheless some results of general kind can be applied to the AWC experiments: the designed AWC device will not be able to sufficiently sample all 3D-disturbances that may propagate in the boundary layer. Furthermore some quite interesting results in the nonlinear regime have been obtained by this new approach of data evaluation, namely considering only linear correlations. 




(a) $R e_{\delta_{1}}=1467$, wall distance $y / \delta_{99} \approx 0.27$. The coordinate ranges of $t$ and $z$ have been adapted to those resulting from Konzelmanns DNS.



(b) DNS: $R e_{\delta_{1}}=1457$, wall distance $y / \delta_{99} \approx 0.54$.

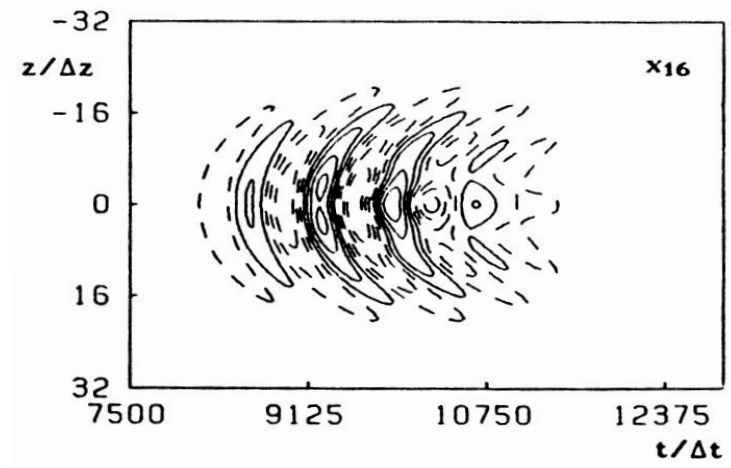

(c) DNS: $R e_{\delta_{1}}=1457$, wall distance $y / \delta_{99} \approx 1.1$.

Figure 7.12: Qualitative comparison between the correlated streamwise velocity fluctuations of the measured pulse and a pulse obtained by a DNS from Konzelmann (1990) (negative values are marked by the dashed isolines). 


\section{Chapter 8}

\section{Active wave cancellation experiments}

This chapter deals with the application of the designed AWC system (Type II). Two control modi are distinguished in order to investigate if the consideration of lateral correlations in the AWC system improves the performance of the system.

The first control modus considers only the streamwise correlation. This is realized by operating independently four SISO system lined up in spanwise direction. Thereby only the transfer functions of the diagonal in the transfer matrices are considered. This modus is therefore denoted as 'independent channels'.

The second modus additionally considers lateral correlations by modelling also the off-diagonal elements of $4 \times 4$ transfer matrices as introduced in chapter 3 . This modus is therefore designated as 'coupled channels'.

\subsection{Application of the MIMO-AWC system to nat- urally occurring disturbance}

Some preparatory work had to be carried out before starting the AWC- experiments. In all of the following experiments the real-time control has been operated at a samplingfrequency of $2 \mathrm{kHz}$. At this sampling rate only half of the possible components in the cancellation device could be used in the real-time control mode in order to avoid overtaxing of the computational power of the used hardware. Thus, the resulting cancellation device consists of 4 reference sensors, 4 actuators and 4 error sensors.

Electrically induced noise has been cleared from all eight sensor signals as described in section 5.4. Each of the eight necessary ENR filters (40 filter taps each) has been 
adapted off-line, i.e. before the real-time AWC was started.

Similarly, all necessary secondary path estimates $S_{i j}$ have been measured beforehand by the system identification scheme. Here, one after the other of the actuators has been fed a white noise signal and adaptive filters (256 filter taps each) modelled the propagation to the error sensors, including the electrical paths of the actuator and of the sensor. For its fast convergence in comparison to the LMS algorithm the sFTF algorithm was used to estimate the secondary path transfer functions. In the case of 'independent channels' only four estimates have been needed, whereas for the 'coupled channels' all 16 elements of the secondary path transfer matrix $\mathbf{S}$ had to be determined. A set of these 16 estimates $S_{i j}$ is presented on the left side of Fig. 8.1 at the preferred free-stream velocity of $U_{\infty}=18 \mathrm{~m} / \mathrm{s}$. The plotted filter taps collected in each of the figures represent the impulse responses between the indicated actuator and the set of error sensors. The impulse responses are divided by the maximum amplitude of the streamwise impulse response and shifted vertically for the sake of clarity. The lateral extent of the control 'pulse' amplitude indicates the ability of the device to control the disturbances at the neighboring error sensor, too. The neighboring error sensor signals are affected by approximately $1 / 3$ of the streamwise influence. This illustrates the achieved improvements of the controllability with respect to $3 \mathrm{D}$ disturbances in comparison to the Type I actuator where this factor has been only 1/10.

In the real-time AWC, 4 independently working filtered-X LMS have been used to model the streamwise transfer functions between the reference sensors and the corresponding actuators in the control modus of 'independent channels'. The complete transfer matrix $\mathbf{W}$ between the 4 reference sensors and the 4 actuators has been modelled by a $4 \times 4$ MIMO filtered-X LMS in the control modus of 'coupled channels'. The simultaneously adapted filter taps for the latter case are presented on the right side of Fig. 8.3. Even though the off-diagonal elements are obviously different from zero, this does not necessarily mean that they are needed to improve the performance of the system. Due to the simultaneous adaptation, the filters may be adapted by evaluating the same (e.g. streamwise) correlation and in fact simply share the work of minimizing this correlation by the adaptation of the filters. In retrospect, it would have been interesting to compare these filters with filters adapted one after the other starting from the diagonal elements.

Moving on to the application of such system to natural TS-waves and the attainable reductions of the velocity fluctuations in the boundary layer, both control modi have been applied to such disturbances for three different free-stream velocities $\left(U_{\infty}=15\right.$, 16.5 , and $18 \mathrm{~m} / \mathrm{s}$ ). A hot-wire traverse has been measured two centimeters downstream of the error sensors to examine the performance of the AWC and to compare the results of both control modi to the uncontrolled case.

The spanwise RMS-distributions for the three different velocities are presented in 


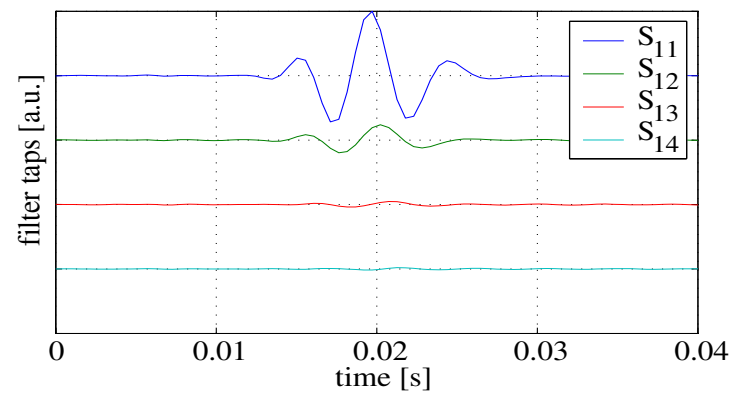

(a) $S_{1 j}$ : actuator at $z=2.5 \mathrm{~cm}$.

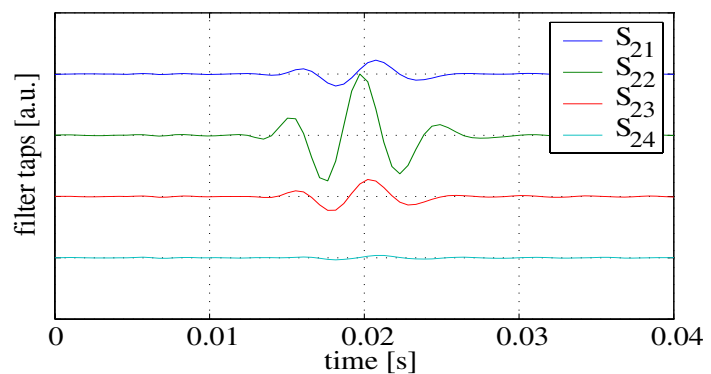

(c) $S_{2 j}$ : actuator at $z=7.5 \mathrm{~cm}$.

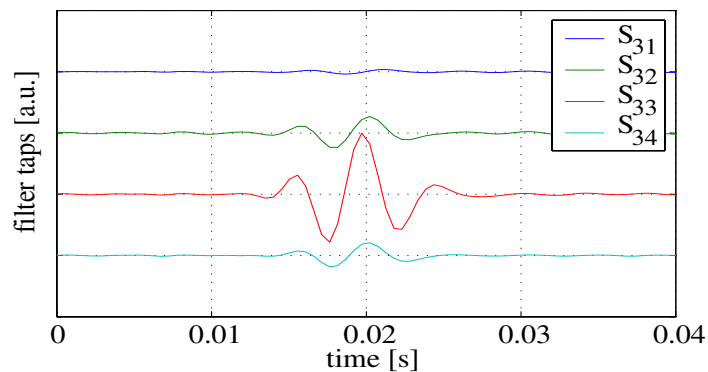

(e) $S_{3 j}$ : actuator at $z=12.5 \mathrm{~cm}$.

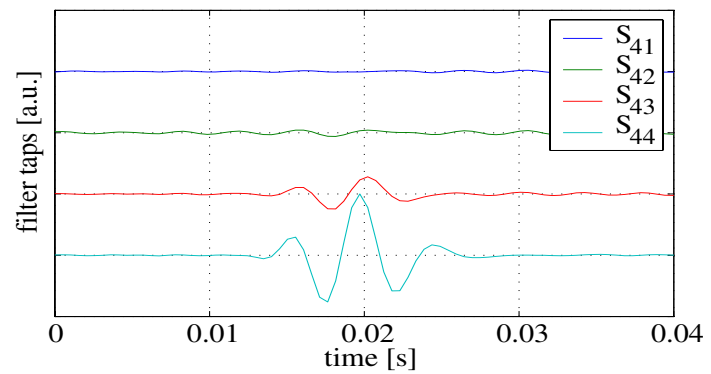

(g) $S_{4 j}$ : actuator at $z=17.5 \mathrm{~cm}$.

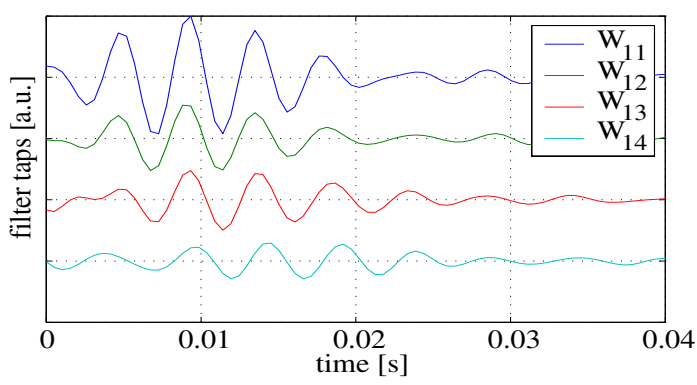

(b) $W_{1 j}:$ reference sensor at $z=2.5 \mathrm{~cm}$.



(d) $W_{2 j}$ : reference sensor at $z=7.5 \mathrm{~cm}$.

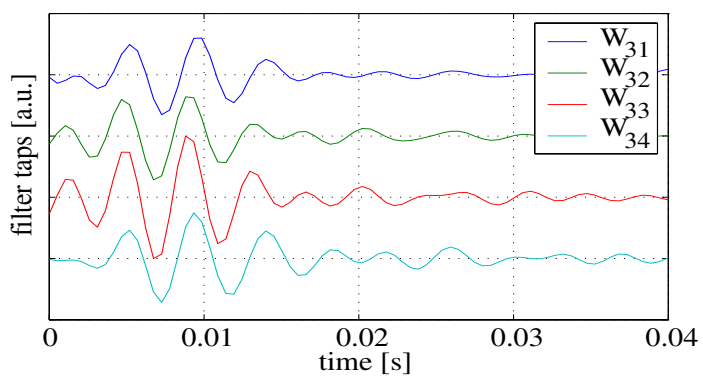

(f) $W_{3 j}$ : reference sensor at $z=12.5 \mathrm{~cm}$.

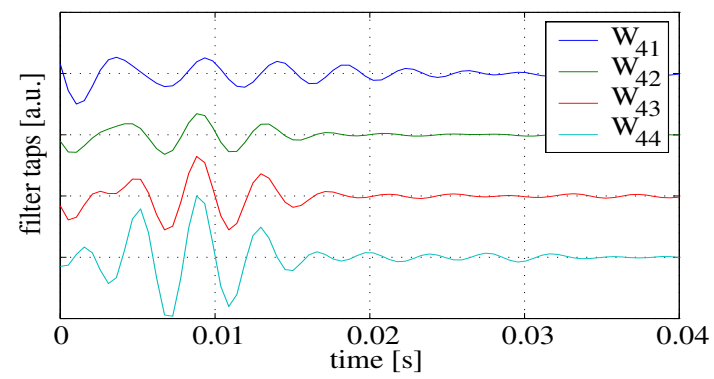

(h) $W_{4 j}$ : reference sensor at $z=17.5 \mathrm{~cm}$.

Figure 8.1: Adapted filters modelling the transfer matrix $\mathbf{S}$ between the actuators and the error sensors (left) and the transfer matrix between the reference sensors and the actuators (right) at $U_{\infty}=18 \mathrm{~m} / \mathrm{s}$. 


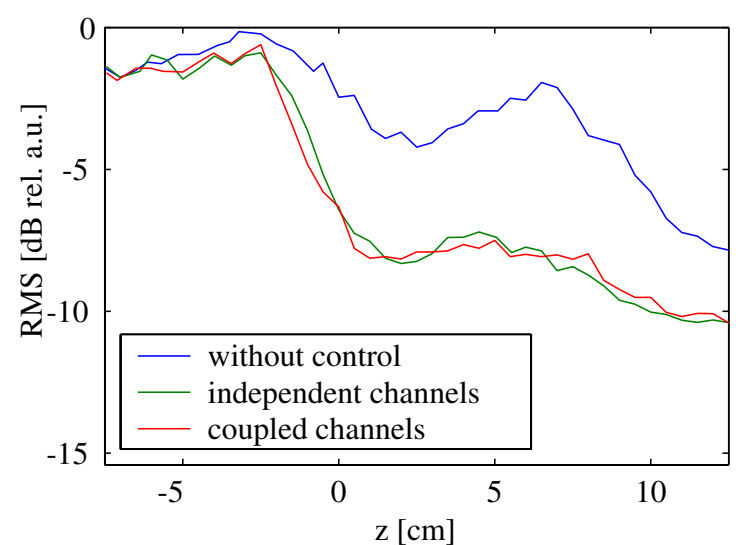

(a) Spanwise RMS distribution (100$210 \mathrm{~Hz})$ at $U_{\infty}=15 \mathrm{~m} / \mathrm{s}$



(c) Spanwise RMS distribution (120$280 \mathrm{~Hz})$ at $U_{\infty}=16.5 \mathrm{~m} / \mathrm{s}$

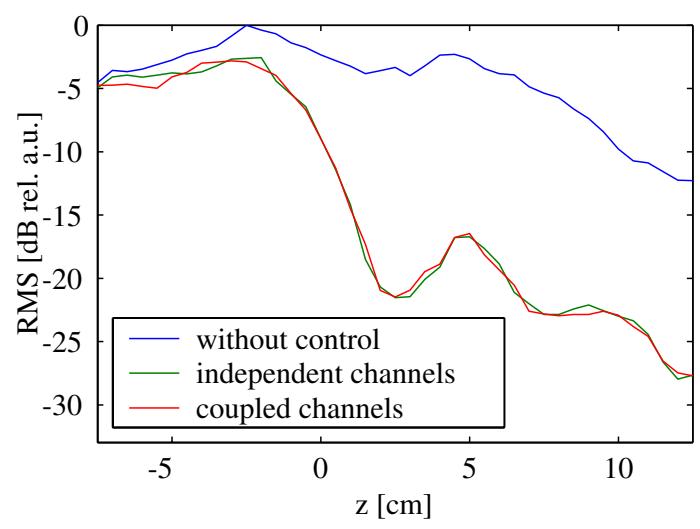

(e) Spanwise RMS distribution (200$300 \mathrm{~Hz})$ at $U_{\infty}=18 \mathrm{~m} / \mathrm{s}$

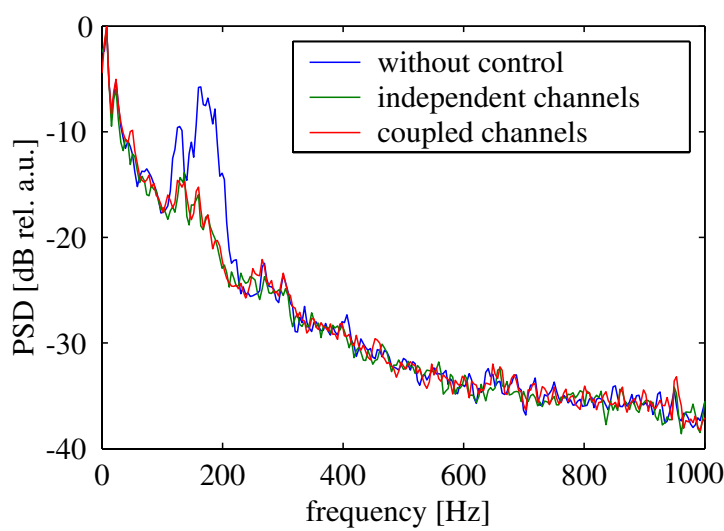

(b) Hot-wire spectrum at $z=2.5 \mathrm{~cm}$, $U_{\infty}=15 \mathrm{~m} / \mathrm{s}$

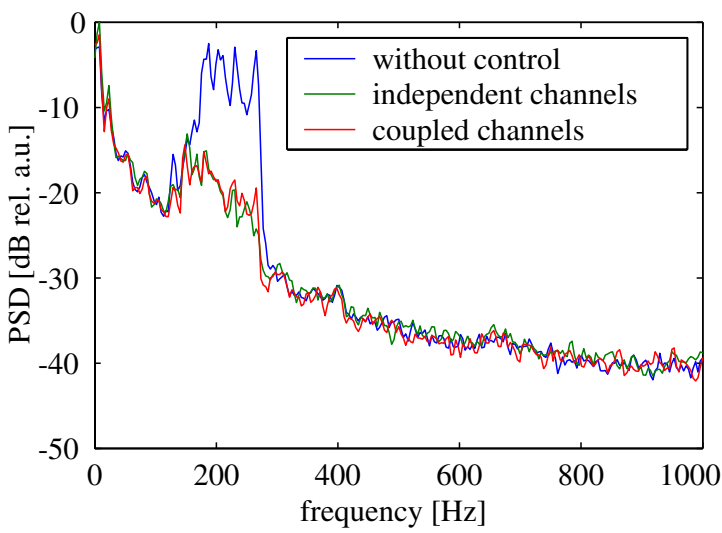

(d) Hot-wire spectrum at $z=2.5 \mathrm{~cm}$, $U_{\infty}=16.5 \mathrm{~m} / \mathrm{s}$

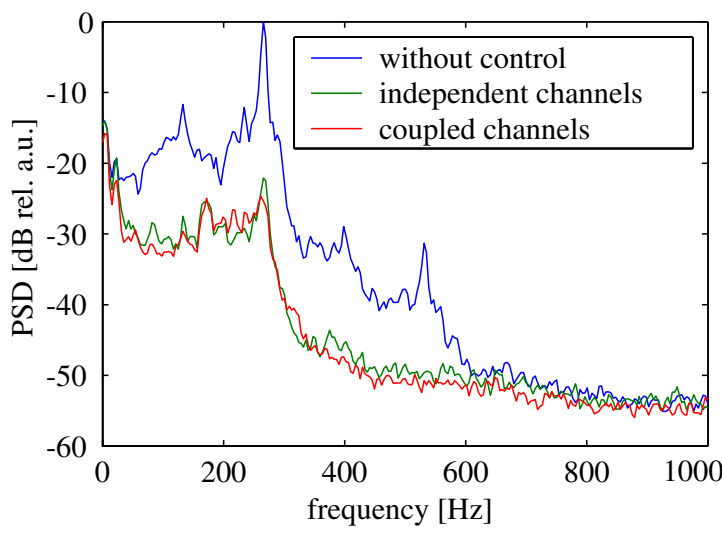

(f) Hot-wire spectrum at $z=2.5 \mathrm{~cm}$, $U_{\infty}=18 \mathrm{~m} / \mathrm{s}$

Figure 8.2: Application of the AWC system to naturally occurring TS-waves operating in two different control modi. 


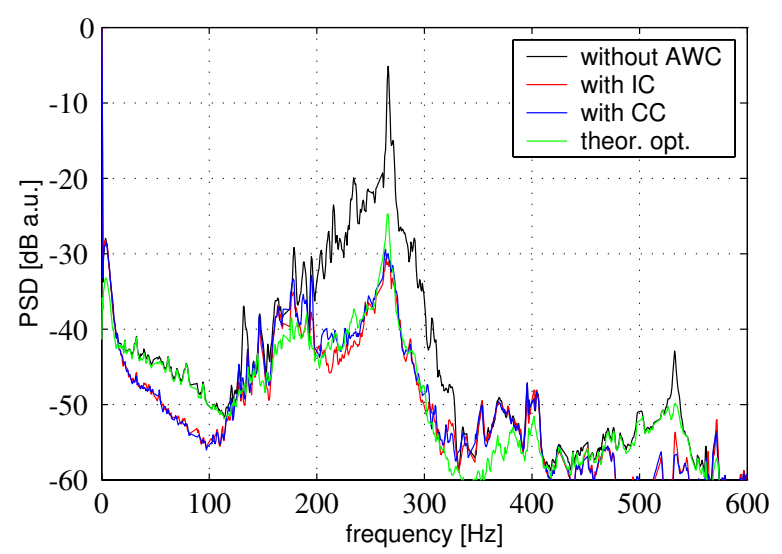

(a) Error sensor $(z=7.5 \mathrm{~cm})$ spectra.



(b) Streamwise coherence between the reference sensor and error sensor at $z=7.5 \mathrm{~cm}$.

Figure 8.3: Spectra and coherence showing the performance of the AWC applied to natural TS-waves.

Fig. 8.2(a, c, e). The error sensors used by the system are located at $z=2.5,7.5,12.5$, and $17.5 \mathrm{~cm}$. Thus, the left side of the figures $(z<0)$ show an uncontrolled region, where the spanwise extent of the AWC becomes visible. The left-most actuator ends at $z=0 \mathrm{~cm}$. The curved shape of the spanwise RMS-distributions is caused by the high receptivity of the point-source slot at $x=20 \mathrm{~cm}, z=0$. No significant differences between both control modi are visible in the spanwise RMS-distribution.

This is also true for the spectra obtained by the movable hot-wire sensor measuring velocity fluctuations at the spanwise position $z=2.5 \mathrm{~cm}$ (see Fig. $8.2(\mathrm{~b}, \mathrm{~d}, \mathrm{f})$ ). It becomes also obvious that the spectra are dominated by single frequency components that are likely to originate from acoustic or vibrational resonances to be discussed below. In the case of $U_{\infty}=18 \mathrm{~m} / \mathrm{s}$ a frequency component at $267 \mathrm{~Hz}$ dominates the whole spectrum. Due to its high amplitude a subharmonic $(134 \mathrm{~Hz})$, a first harmonic $(534 \mathrm{~Hz})$, and even a peak at $400 \mathrm{~Hz}(=267 \cdot 3 / 2 \mathrm{~Hz})$ is generated. One could assume that the propagation of TS-waves is dominated by nonlinear effects, so that the linear coherence between reference and error sensor is degraded. The coherence between the wall shear stress signals of the reference and error sensor at $z=7.5 \mathrm{~cm}$ is plotted in Fig. 8.3(b). It reaches values up to 0.99 in the case 'without AWC' and is reduced by both control modi in the range between 200-300 Hz. Nevertheless large parts of the coherence have not been affected by the AWC. The remainders are concentrated in the frequency range $100-200 \mathrm{~Hz}$ and $350-400 \mathrm{~Hz}$. Also the coherence of the $267 \mathrm{~Hz}$ component does not drop below approximately 0.8 , although the AWC is activated. 


\begin{tabular}{||c||c|c|c|c|c||}
\hline$\gamma_{r e}^{2}$ & 0.1 & 0.3 & 0.5 & 0.7 & 0.9 \\
\hline$\sigma\left(\gamma_{r e}^{2}\right)$ & 0.0232 & 0.0313 & 0.0289 & 0.0205 & 0.0077 \\
\hline
\end{tabular}

Table 8.1: The standard deviation for different values of the coherence function $\gamma_{r e}^{2}$ evaluated for $n_{a v}=300$.

The relatively large variations of the coherence function $\gamma_{r e}^{2}$ can partially be explained by the small number of averages $n_{a v}=300$ (corresponding to approximately 8 minutes of measuring time). The related standard deviation $\sigma\left(\gamma_{r e}^{2}\right)$ can be approximated by

$$
\sigma\left(\gamma_{r e}^{2}\right) \approx \sqrt{2}\left|\gamma_{r e}\right|\left(1-\gamma_{r e}^{2}\right) / \sqrt{n_{a v}}
$$

(Bendat 1978). This equation has been evaluated for different values of the coherence with $n_{a v}=300$. The result is shown in table 8.1. The standard deviation is large for moderate values while it becomes small for $\gamma_{r e}^{2} \approx 1$ and $\gamma_{r e}^{2} \approx 0$. Therefore the coherence plots presented here cannot be interpreted with respect to every detail. Nevertheless, some of the details were repeatable and thus can be interpreted. The spectra measured directly at the error sensor $(z=7.5 \mathrm{~cm})$ for $U_{\infty}=18 \mathrm{~m} / \mathrm{s}$ are presented in Fig. 8.3(a) and also indicate no significant difference between the control modi. The obtained reduction of the $267 \mathrm{~Hz}$ component as well as the subharmonic and the harmonic part is higher than expected from the theoretical optimum ('theor.opt.') that is calculated from the streamwise coherence only. These deviations can be explained by the degradation of the coherence between the reference and the error sensor, e.g. by the amplitude dependent nonlinearity; one should keep in mind that in this case the coherence between the reference sensor and the signal at the location of the actuator determines the highest possible attenuation.

To summarize the results from this section: The ability of the redesigned actuator (Type II) to control disturbances also at neighboring error sensors has been demonstrated. Furthermore, the AWC system has been operated in both control modi achieving a reduction of up to $22 \mathrm{~dB}$ (RMS), which however does not significantly differ between the two control modi.

A possible reason for this unexpected finding could be that the excited wavenumber spectrum of the natural TS-waves is dominated by a 2D-part and therefore no need exists to distinguish between different spanwise wavenumber components propagating in the wave field. Moreover the frequency depending parts of the coherence between reference and error sensor that remain in spite of the counter-action of the AWC may be related to the missing difference in the performance of both control modi. A further reason may have been the spatial sampling of the natural TS-wave field which has turned out to be insufficient for some of the components as found out by the pointsource measurements presented in the previous chapter. 


\subsection{The spatial spectrum of naturally occurring disturbances}

In order to investigate the natural disturbance wave field two-point correlation measurements have been carried out to determine the spanwise correlation of the disturbances the AWC system is confronted with. A movable hot-wire probe has been traversed $2 \mathrm{~cm}$ downstream of the reference sensor array. The coherence between each of the 4 reference sensors and the movable hot-wire probe has been measured at each spanwise position of the movable hot-wire. The resulting spanwise coherence function is shown in Fig. 8.4 (a). It becomes obvious that the spanwise coherence strongly depends on the temporal frequency. For some single frequency components the coherence does not significantly decrease within the reach of the measurement $(\Delta z=22.5 \mathrm{~cm})$. Similarly, the spanwise coherence function at the error sensor array has been obtained and is plotted in Fig. 8.4(b). The arrow labelled by '1' marks the frequency component of approximately $270 \mathrm{~Hz}$ which is highly correlated in spanwise direction and is the component that dominates the error sensor spectrum in Fig. 8.2 (f) and Fig. 8.3 (a). Even though this component is also present in the spanwise coherence at the reference sensor array, it is not as dominating as another component at a slightly lower frequency $(255 \mathrm{~Hz})$. As mentioned above these components are very likely caused by vibrations of the plate itself which will be confirmed later.

Furthermore, nonlinear effects become visible in the spanwise coherence indicated by

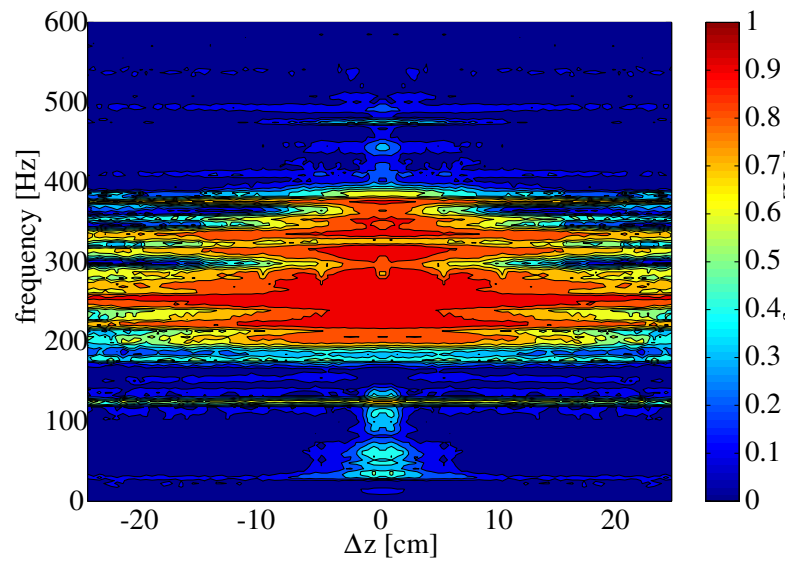

(a) Reference sensor

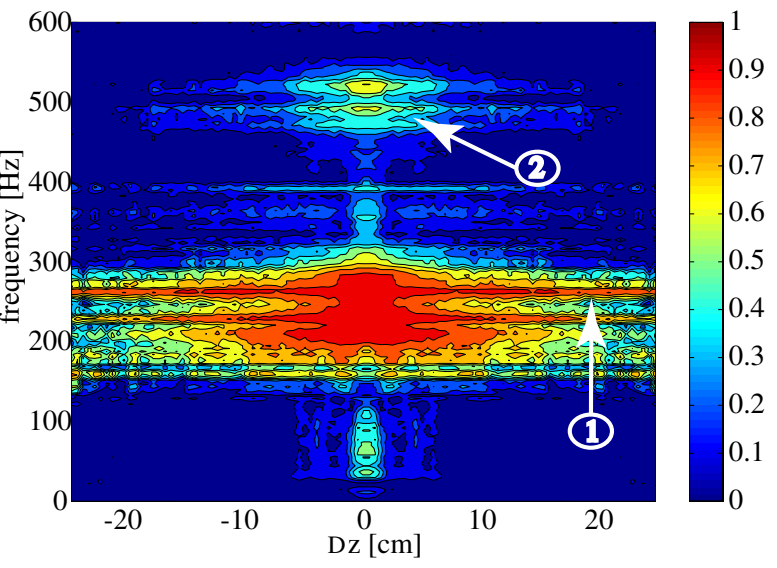

(b) Error sensor

Figure 8.4: Spanwise coherence of naturally occurring disturbances. 


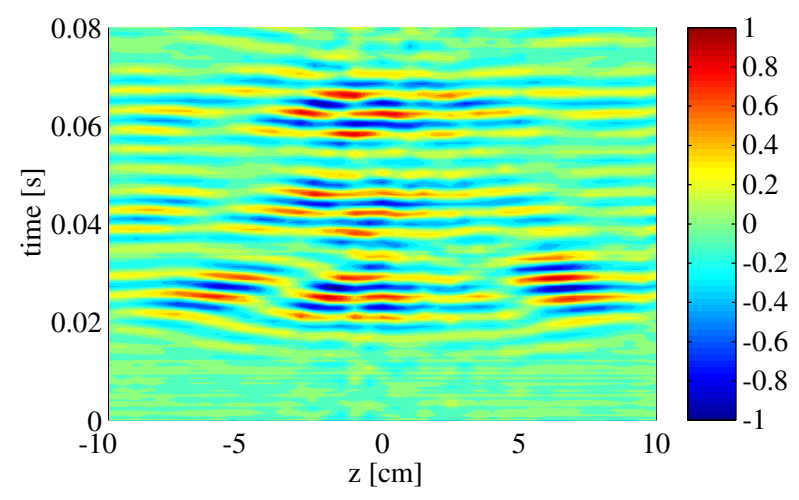

Figure 8.5: Spatial impulse response between the acoustic source in the settling chamber of the wind tunnel and a spanwise traversed hot-wire probe, illustrating the acoustic receptivity of the point-source slot $(z=0 \mathrm{~cm})$. The colors indicate the pulse amplitude.

the second-harmonic part marked by '2'. Three dimensional components generated by these nonlinear effects, similar to the one observed in the point-source experiment may be responsible for the decrease in lateral coherence when the disturbances travel further downstream. This is unexpected if one considers the spanwise spreading of disturbances that are governed by the linear dispersion relation as outlined in section 3.1. Apart from these artifacts impairing the present results, it can be concluded that the natural TSwaves the AWC system is confronted with are highly correlated in spanwise direction.

Assuming that these natural TS-waves are generated mainly by sound in the wind tunnel the following experiment may give some further insight into the disturbance spectrum the AWC system is exposed to. A loudspeaker has been placed in the settling chamber of the wind tunnel as excitation source. The source has been driven by a white noise signal and a sound pressure of about $112 \mathrm{~dB}$ has been generated as measured by a microphone close to the leading edge of the model plate.

Again, a movable hot-wire probe has been traversed $2 \mathrm{~cm}$ downstream of the error sensors to measure the impulse response that corresponds to the transfer function between the excitation signal of the loudspeaker and the hot-wire signal. This has been achieved by applying the system identification scheme similarly to the point source experiment described in the previous chapter. Again, the impulse responses obtained at the various spanwise positions have been assembled. They show a kind of scattered pulse indicating that such acoustic perturbations have been transformed to disturbances propagating within the boundary layer (see Fig. 8.5).

The pattern shows three pulses that are centered around $z=0 \mathrm{~cm}$, which is the spanwise position of the open slot of the point-source. This leads to the assumption that the high acoustic receptivity of the slot leads to the generation of these pulses 
driven externally by the sound pressure fluctuations. The appearance of three pulses is likely to be caused by acoustic echoes. Due to the complex geometry of the wind tunnel the acoustic echoes cannot be identified directly. However, the delays between the pulses match the order of magnitude expected from the dimensions of the wind tunnel. Additionally, the pulses are interfering with 2D-waves that are generated at a $0.1 \mathrm{~mm}$ thick technical 2D roughness caused by the tape covering the disturbance generators.

A comparison between the spatial Fourier transforms of the measured transfer functions and the one obtained in the point-source experiment is presented in Fig. 8.6.

The shapes of the spanwise wavenumber spectra of Fig. 8.6 (a) \& (b) exhibit a similarity of the affected region in the spectra. For sake of clarity, the white line indicates the silhouette of the spectrum in Fig. 8.6 (b) where the high wavenumber components are indicated as 'ears'. Due to the noise underground in the spectra, the color scale in the figures was limited to $-30 \mathrm{~dB}$. As a result of this limitation the aforementioned 'ears' are not visible in Fig. 8.6 (a), but they can be anticipated from the raw data.

The frequency spectra at the zero wavenumber component can be compared in Fig. 8.6 (c). The RMS wavenumber spectra of both experiments are shown in Fig. 8.6 (d). Both the spectra have a nearly identical broadband part that is caused by the acoustic receptivity of the point source which is driven by the internal loudspeaker and the external sound pressure, respectively. The strong 2D component superimposed on the spectrum resulting from the loudspeaker in the settling chamber originates mainly from the acoustic receptivity of the aforementioned 2D technical roughness.

The presented RMS-spectra therefore reveal that the broadband part that is to be attenuated by the AWC system, is burried in a $12 \mathrm{~dB}$ stronger $2 \mathrm{D}$ part. Another drawback that becomes obvious is the fact, that the broadband part of the wavenumber spectrum extends far beyond the Nyquist-wavenumber $\left(\beta_{N y}^{*}=0.628 \mathrm{~cm}^{-1}\right.$ in the Type II control system). So these disturbances have been insufficiently sampled and therefore could not be suppressed very efficiently even if the full 3D cancellation capacity of the system is switched on. 


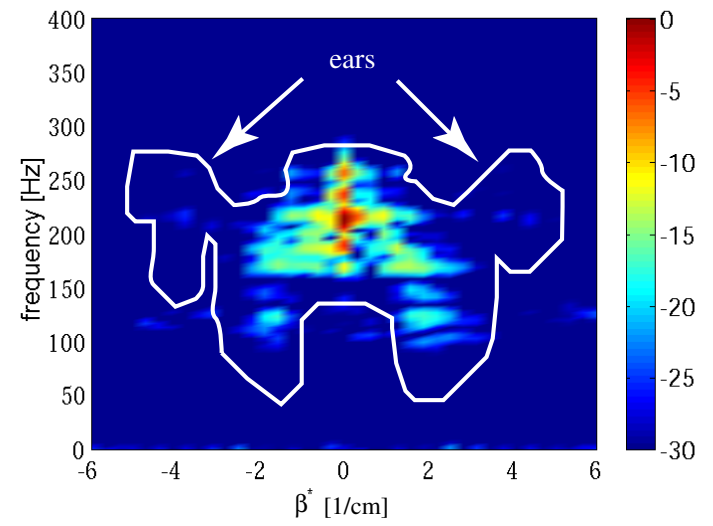

(a) Spanwise wavenumber spectrum of the correlated boundary layer disturbances generated by acoustic excitation. The white line indicates the silhouette of the spectrum in Fig. (b).

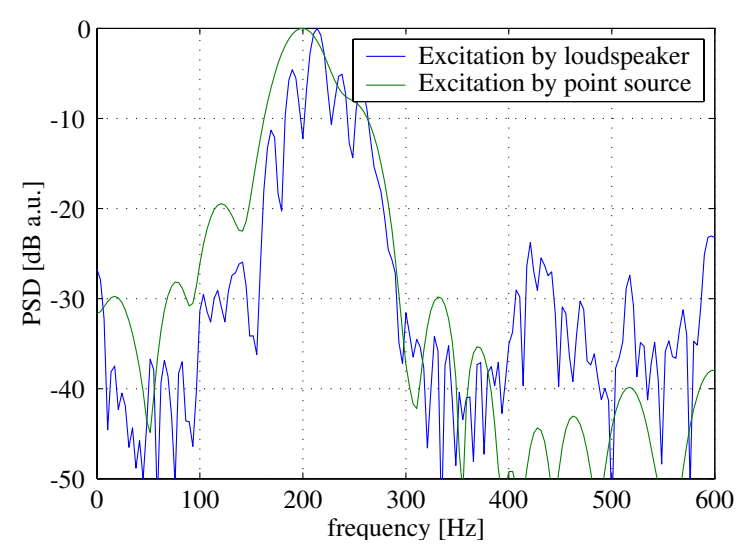

(c) Frequency spectra for $\beta^{*}=0$.



(b) Spanwise wavenumber spectrum of the correlated boundary layer disturbances generated by point-source excitation.

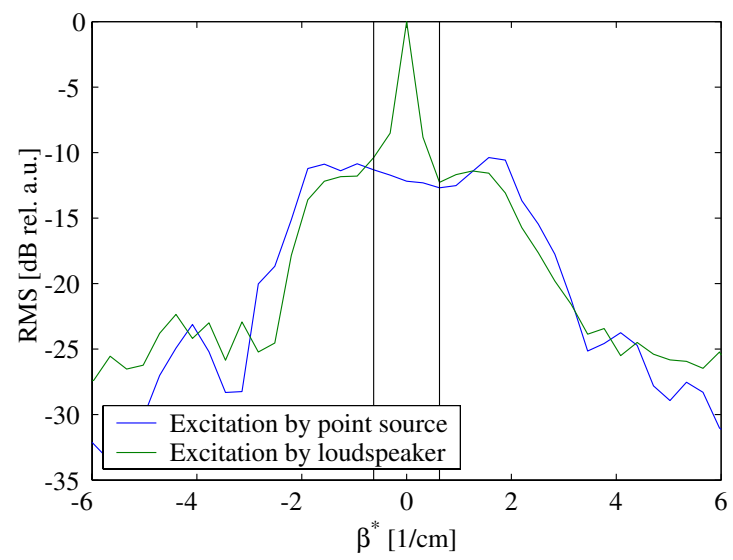

(d) RMS-wavenumber spectra (100-300 Hz)

Figure 8.6: Comparison between the excitation by the point source and the one by a loudspeaker. 


\title{
8.3 The application of the MIMO AWC system to broadband point-source disturbances
}

\begin{abstract}
Remark:
Due to some modifications of the set-up the trailing edge flap has been slightly misaligned. Therefore the experimental conditions in the experiments described below have not been exactly the same as before. The resulting favorable pressure gradient at the rear end of the plate had a slightly stabilizing influence on the boundary layer which is indicated by the lower disturbance level measured at the error sensors.
\end{abstract}

In order to gain deeper insight into the effects associated with the lateral coupling of the streamwise flow-control channels, another cancellation experiment was carried out: The disturbances to be cancelled were excited by the point-source, and again, the cancellation system was operated in the two different control modi and compared with the uncontrolled case. The impulse responses between the excitation signal and the hot-wire signal measured at a traverse $2 \mathrm{~cm}$ downstream of the error sensors are plotted in the figures 8.7 (a), (c) \& (e). The filters used in both control modi have been adapted simultaneously while exposed to the point-source disturbances.

The spanwise position of the point source is at $z=0 \mathrm{~cm}$, and the spanwise positions of the error sensors are given by the arrows. Because of the localized excitation of the disturbances the oblique waves with higher values of $\beta$ are found at higher spanwise coordinates. The system consisting only of independent streamwise channels reduced the amplitude of the pulse quite well in the inner region, whereas in the outer region, damping is found only close to the location of the right-most error sensor. Only a few millimeters aside the disturbances are even amplified. This can be explained by the fact that the system tries to cancel the $3 \mathrm{D}$ wave by a $2 \mathrm{D}$ wave. A closer look reveals that the affected spanwise position does not exactly match the position of the error sensor but is shifted by approximately $7 \mathrm{~mm}$ to the center. This can be explained by the difference in the wall-normal eigenfunctions between the 2D-wave generated to cancel the $3 \mathrm{D}$ wave and the 3D wave itself. The AWC system measures and tries to cancel only the wall-shear stress fluctuations detected at the wall-hot-wire sensors, whereas the presented impulse response is based on the measurement of velocity fluctuations in the boundary layer above.

The control system with coupled channels showed a wider region of damping at the error sensors but less damping in the inner region. The observed shift of the affected region can be found here as well, but is not as distinctive as in the case of the 'independent channels'.

The related wavenumber spectra for the three cases are shown in the figures 8.7 (b), (d) \& (f). The white lines in the figures indicate the Nyquist wavenumber 
of the AWC system. The wavenumber spectrum in the case of 'independent channels' reveals that a single component is reduced by approximately $25 \mathrm{~dB}$ whereas the remaining spectrum is much less affected. The affected component does not exactly match the zero-wavenumber component as expected, but has a slightly positive wavenumber. The reason for this is found in the asymmetry of the disturbance pulse that may be caused by a small spanwise variation in the pressure gradient.

The affected part of the wavenumber spectrum when the lateral coupling is switched on extends to the Nyquist wavenumber of the AWC system. However, an overall reduction of only $17 \mathrm{~dB}$ is obtained in this case. It could be assumed that this difference in maximum reduction might be due to insufficient sampling. Because of the decreasing sensitivity of the wall shear stress sensors to 3D waves of higher inclination angles, this is found to be unlikely. On the other hand, it can originate from the fact that the cancellation power of the system is concentrated to one single wavenumber component in the first case while it is distributed over a whole spectrum of wavenumbers in the second case. In any case the total power of the remaining disturbances as seen by the error sensors is decreased by the lateral coupling of the channels.

However, the presented transfer functions measured across the cancellation device revealed the capability of the designed control device to cancel real 3D disturbances containing all possible frequency and wavenumber components. The filters used in both control modi have been adapted simultaneously while exposed to the point-source disturbances. Due to the different spatial disturbance distribution in comparison to the natural disturbances the presented results can not be transferred directly to the case of natural TS-waves, but show the general capability of the designed cancellation device to deal with 3D disturbances. 


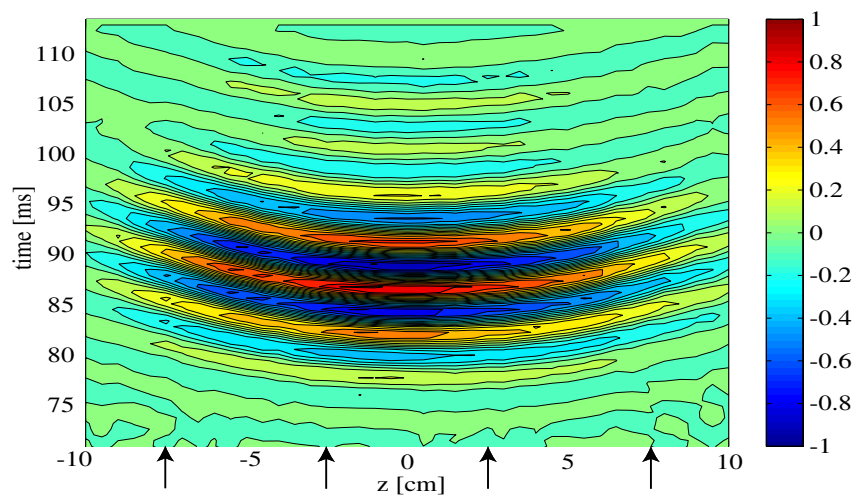

(a) Uncontrolled point source disturbance

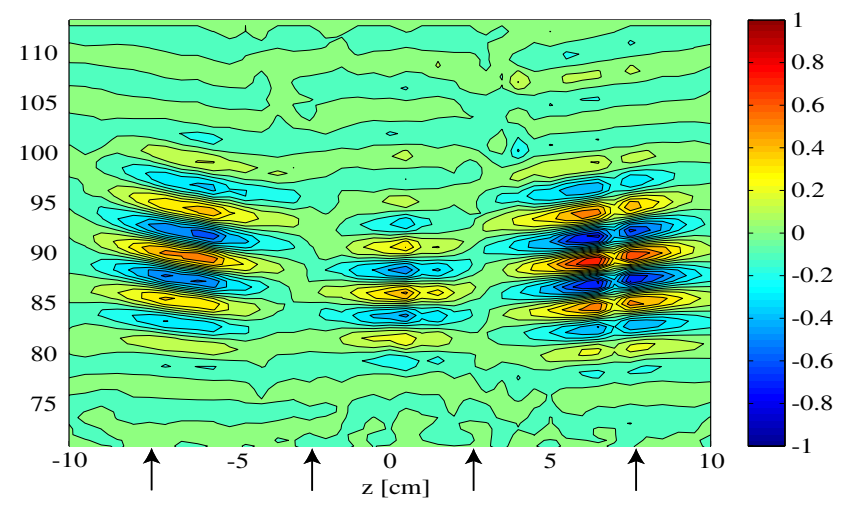

(c) Point source disturbance controlled by independent channels

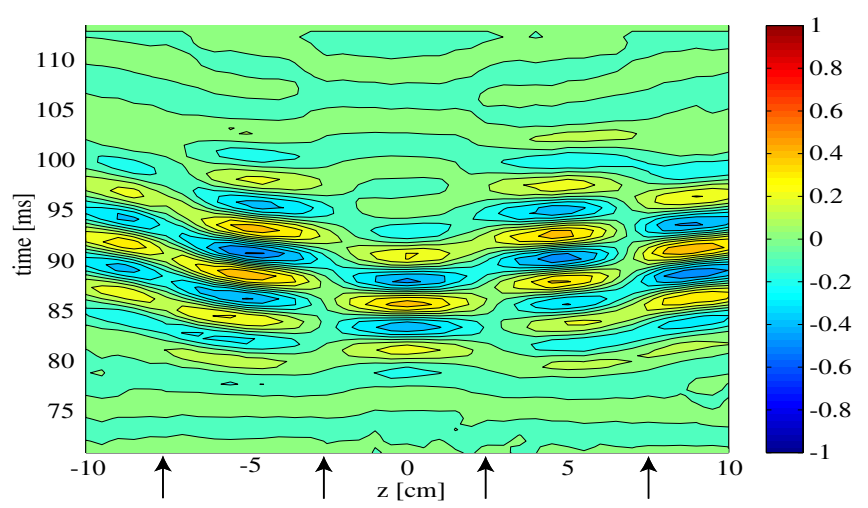

(e) Point source disturbance controlled by coupled channels



(b) Uncontrolled point source disturbance

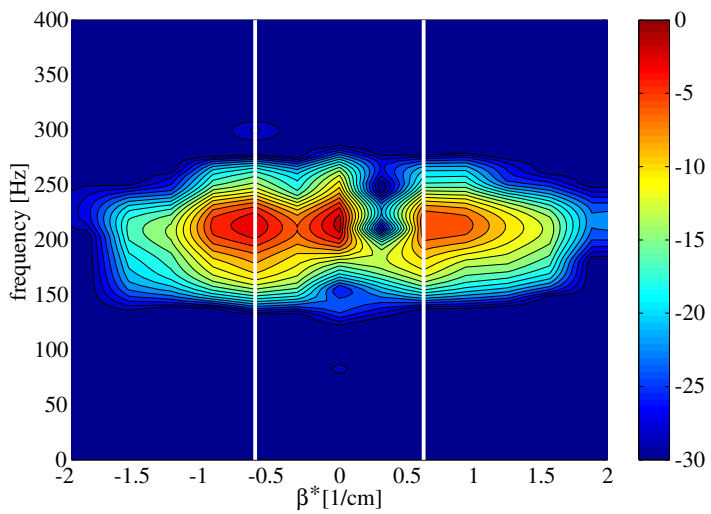

(d) Independent channels

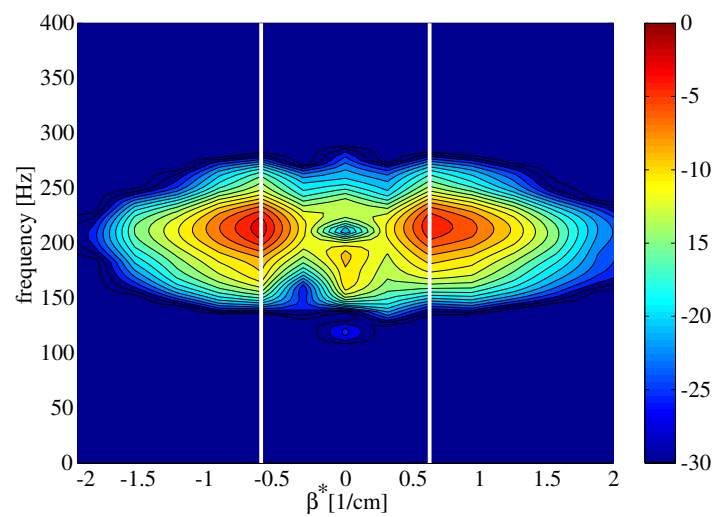

(f) Coupled channels

Figure 8.7: Left side: Spatial pulse of a point source disturbance measured at $x=74 \mathrm{~cm}$ for $U_{\infty}=18 \mathrm{~m} / \mathrm{s}$ uncontrolled (a), controlled by independent channels (c), controlled by coupled channels (e), and the related spanwise wavenumber spectra (right side). 


\section{Chapter 9}

\section{Acoustic and vibrational influences on the control system}

This chapter deals with acoustic effects on the active wave cancellation. It turned out during the investigations that the interactions between TS-waves, sound and vibrations lead to rather complex correlations between the considered signals. These strongly depend on the environmental conditions and will therefore change with varying experimental parameters e.g. flow velocity etc., and strongly differ between different experimental set-ups. Thus, the correlation data presented in this chapter will not be discussed in every detail, but the most relevant relations and physical processes leading to these correlations will be dealt with. Last but not least the resulting consequences for the AWC system will be presented.

However, there is also a practical relevance of theses effects. Due to e.g. wing vibrations and propeller or turbine noise, it is very likely that these effects play a key-role under free-flight conditions, too.

\subsection{Modification of the experimental set-up}

In order to simplify the identification of the relevant processes as well as to reduce the amount of data to be evaluated, the AWC system has been reduced to a SISO-system. This single-input-single-output system consists of one reference sensor, one actuator and one error sensor all placed at the same spanwise position $(\mathrm{z}=-2.5 \mathrm{~cm})$.

A microphone has been placed at the lower end of the measurement region outside the boundary layer to obtain data for the correlation between the acoustical pressure fluctuations and the sensor signals. The microphone was equipped with a wind-conus to avoid noise caused by flow separation and it was mounted at the streamwise position of 
the actuator slot. The spanwise distance has been chosen as $30 \mathrm{~cm}$ to avoid influences on the boundary layer at the AWC-device.

Furthermore an accelerometer has been glued to the plate close to the position of the microphone in order to evaluate also the correlation between the wall-hotwire signals and vibrations of the plate. The accelerometer has been insulated to perturbations caused by the flow by a non-contact cover and by fixation of the signal-cable to the plate.

All correlations between the signals of the reference and error sensor as well as the signals from the microphone and accelerometer have been evaluated by the 8-channel FFT-analyzer COMPASS (Gould-Nicolet), whereas the presented impulse responses are determined via the system identification scheme.

\subsection{SISO AWC system in a noisy environment}

In order to prepare this cancellation experiment, the secondary transfer function $S$ was estimated off-line by driving the actuator with a white noise signal and adapting a FIRfilter between the driving signal and the error sensor signal (ES) measured by the wall hot-wire. The obtained impulse response (as represented by the filter taps) is shown in Fig. 9.1 (a). Besides the TS-wave that is generated directly by the actuator (A) a second 20 times smaller pulse at a delay of about $0.084 \mathrm{~s}$ indicates the aforementioned sound induced TS-wave generated at the unevenness remaining from the former disturbance generator $(\mathrm{DG})$.

For comparison the impulse response $R$ measured between the actuator and the reference sensor $(\mathrm{RS})$ is plotted in the same figure. As expected, the sound induced TS-wave is here also visible as well. The estimated delay $\mathrm{d} T$ (marked in the figure) matches the difference between both propagation paths and equals therefore the time needed by TS-waves to travel along the streamwise distance $\mathrm{d} x=22 \mathrm{~cm}$ between the reference and error sensor (see No. 2 in Tab. 9.1).

The frequency dependent group velocity of TS-waves that has previously been estimated to approximately $40 \%$ of the free-stream velocity matches the envelope velocity determined to $40.6 \%$ quite well. Taking the caused delay into account and neglecting the delay caused by the sound propagation, the estimated delay $d T$ can be expressed in terms of a travelling distance and thus can be compared to the expected value. The results have been summarized in the table 9.1 for a variety of processes identified on the basis of the correlation data. The obtained results may slightly differ from the expected values due to a number of possible influences, e.g. the frequency dependence of the group velocity and possible changes due to the observed small changes in the streamwise pressure gradient (see Fig. 7.1). Furthermore the envelope method is not 


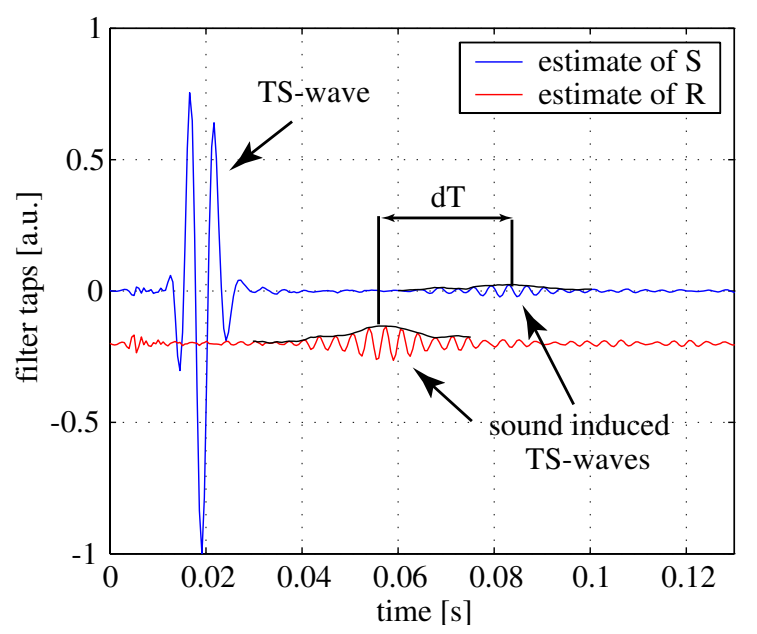

(a) The impulse responses of the estimated secondary and feedback transfer function $S$ and $R$ (shifted downwards by -0.2 and multiplied by 3 ).

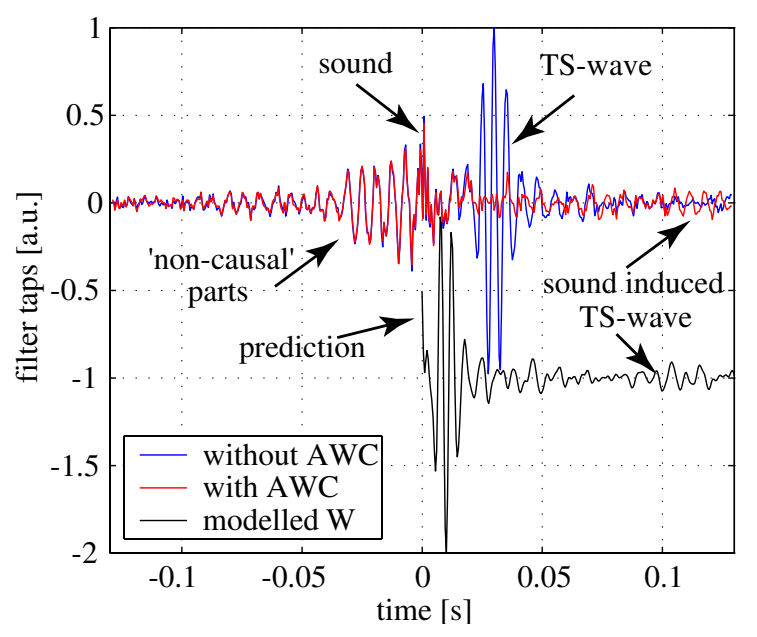

(c) Impulse responses between the reference and error sensor $H_{r e}$ with and without AWC. The modelled control impulse response $W$ is shifted by -1 .

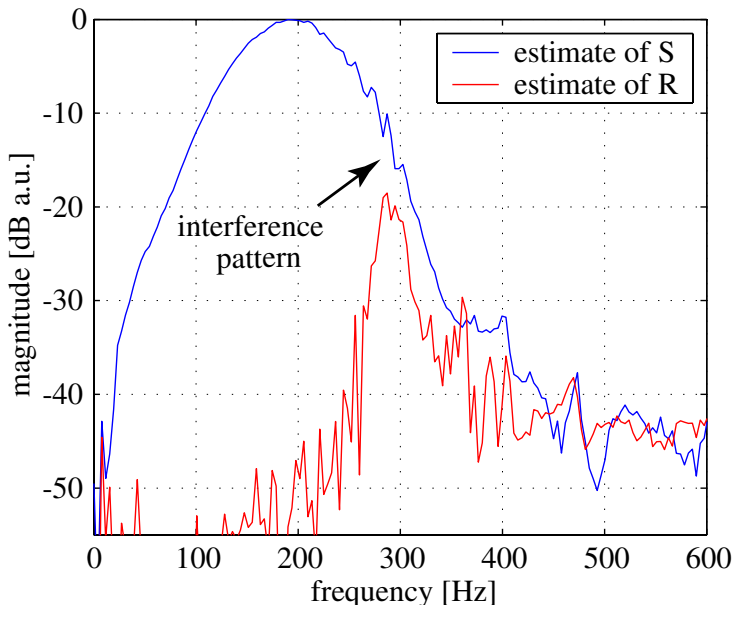

(b) Magnitude of the transfer functions $S$ and $R$.

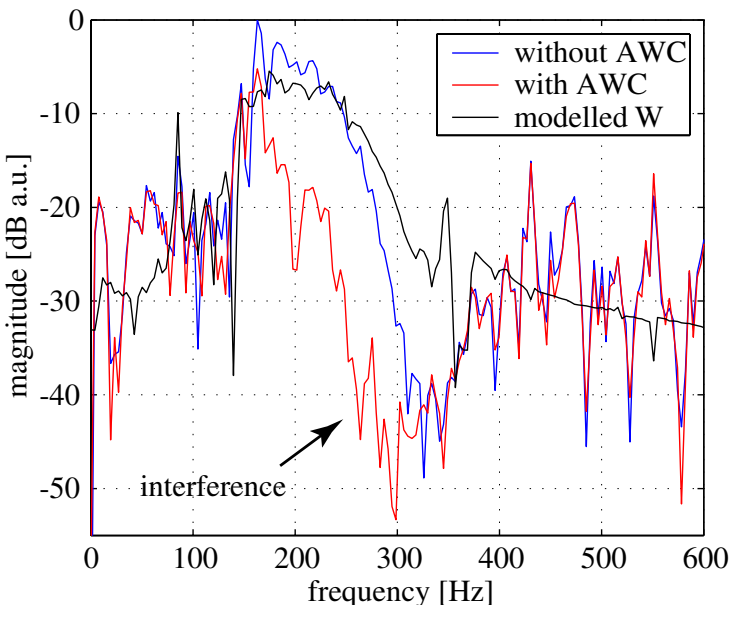

(d) The magnitude of the transfer functions $H_{r e}$ with and without AWC; and the modelled $W$.

Figure 9.1: The influence of sound and vibrations on the modelling by a SISO-AWC system. 
very precise for narrow-band 'pulses', e.g. the sound induced TS-waves mentioned above. Moreover interference between different processes may impair the results. Despite these error sources the estimated travelling distances agree quite well with the theoretically expected values.

Fig. 9.1 (b) shows the magnitude of the estimated transfer functions $S$ and $R$. The interference patterns marked in the figure indicate the frequency range $(250-320 \mathrm{~Hz})$ where this acoustically induced feedback may influence the AWC. The drop-off to lower frequencies observed for the magnitude of $R$ is likely to be caused by the decrease of sound radiation at low frequencies. In order to get a proper estimate $\hat{S}$ for the control model the FIR filter used has been shortened in the way that only the primary TS-pulse is modelled (0.04 s of the impulse response).

After the modelling of $\hat{S}$ the main filter $W$ was adapted. The impulse response is shown as the black curve in Fig. 9.1(c). In order to survey the performance of the control the impulse response $H_{r e}$ between the reference sensor (RS) and the error sensor (ES) has been measured and plotted into the same figure for comparison. Here also negative time units are considered to check whether correlated components are measured first at ES before they are detected at RS. The blue curve shows the $H_{r e}$ without AWC and the red one $H_{r e}$ with the AWC switched on.

The component caused by the TS-wave propagating downstream is therefore detected first at RS and 0.029 seconds later at ES (see No. 4 in Tab.9.1). This part is well modelled by the adaptive filter $W$ (counter-phase) and therefore reduced by more than $20 \mathrm{~dB}$. The difference in delay of the original and the modelled TS-impulse $(0.019$ seconds) is mainly caused by the delay of the lowpass filters.

Due to the sound radiation of the actuator during cancellation a smaller pulsecomponent turns up at a delay of 0.115 seconds which is caused by the sound induced TS-wave (see No. 4 in Tab. 9.1). This component is also modelled by $W$ but without sign reversal as expected, which becomes obvious from Fig. 9.1 (c) when $W$ (black) is shifted such that the main pulse corresponds to the one in $H_{r e}$ (blue).

However, it is not fully suppressed which may have been caused by interference due to other processes.

The part of $H_{r e}$ at delay zero is caused by sound detected by the sensors. This assumption is supported by other measurements presented later in this chapter.

The components at negative time units are denoted as 'non-causal' parts in the figure, because from the point of view of the feed-forward control system the effect is observed before its cause. The components at small positive time units may also be considered as 'non-causal' in this sense but they can be minimized by reducing the delay of the relevant lowpass filters. The modelled filter $W$ shows some kind of prediction behavior to cope with these 'non-causal' components by increasing the first 


\begin{tabular}{||c|c|c|c|c||}
\hline No. & $\begin{array}{c}\text { Source } \\
\text { wave } \\
\text { source } \stackrel{\text { Target }}{\longrightarrow}\end{array}$ & $\begin{array}{c}\text { Target } \\
\text { source } \stackrel{\text { wave }}{\longrightarrow} \text { target }\end{array}$ & $\begin{array}{c}\left\{\int c_{\text {gr }} \mathrm{d} t\right\}_{\text {exp }} \\
{[\mathrm{cm}]}\end{array}$ & $\begin{array}{c}\left\{\int c_{\text {gr }} \mathrm{d} t\right\}_{\text {theo }} \\
{[\mathrm{cm}]}\end{array}$ \\
\hline \hline 1 & $\mathrm{~A}$ & $\mathrm{~A} \stackrel{T S}{\longrightarrow} \mathrm{ES}$ & 10.3 & 11 \\
\hline 2 & $\mathrm{~A} \stackrel{\text { sound }}{\longrightarrow} \mathrm{DG} \stackrel{T S}{\longrightarrow} \mathrm{RS}$ & $\mathrm{A} \stackrel{\text { sound }}{\longrightarrow} \mathrm{DG} \stackrel{T S}{\longrightarrow} \mathrm{ES}$ & 21.9 & 22 \\
\hline 3 & $\stackrel{\text { sound }}{\longrightarrow} \mathrm{DG} \stackrel{T S}{\longrightarrow} \mathrm{RS}$ & $\stackrel{\text { sound }}{\longrightarrow} \mathrm{A} \stackrel{T S}{\longrightarrow} \mathrm{ES}$ & 18.3 & 19 \\
\hline 4 & $\mathrm{RS}$ & $\mathrm{ES}$ & 21.2 & 22 \\
\hline 5 & $\mathrm{RS}$ & $\mathrm{RS} \stackrel{W}{\longrightarrow} \mathrm{A} \stackrel{\text { sound }}{\longrightarrow} \mathrm{DG} \stackrel{T S}{\longrightarrow} \mathrm{ES}$ & 62.7 & 63 \\
\hline
\end{tabular}

Table 9.1: Schematic overview of several propagation processes identified in the correlation data. The experimentally determined $\left(\left\{\int c_{g r} \mathrm{~d} t\right\}_{\text {exp }}\right)$ and the theoretically expected $\left(\left\{\int c_{g r} \mathrm{~d} t\right\}_{\text {theo }}\right)$ travelling distances are compared for the different propagation processes. Abbreviations: DG: disturbance generator, RS: reference sensor, A: actuator, ES: error sensor.

coefficients.

Fig. 9.1(d) shows the magnitude of the transfer functions $H_{r e}$ with and without AWC as well as the one of the modelled $W$. Besides the reduction of $H_{r e}$ by the AWC mainly two properties become obvious. There is only a small reduction attained in the frequency range $(140-200 \mathrm{~Hz})$ centered at about $160 \mathrm{~Hz}$ and there is also a minus $15 \mathrm{~dB}$ depression in the frequency range $300-400 \mathrm{~Hz}$ which is already observed without the AWC switched on.

These observations rise a number of questions. Where do these 'non-causal' components come from? How do they influence the performance of the AWC system? Is it possible to consider them in the AWC model in order to minimize their influence? Similar questions may be asked for the sound induced TS-waves and the sound that is directly measured by the sensors. What causes the incomplete cancellation in the frequency range $140-200 \mathrm{~Hz}$ and what causes the $15 \mathrm{~dB}$ depression in the frequency range $300-400 \mathrm{~Hz}$ ?

The origin of one part of the 'non-causal' components became clear by measuring $H_{r e}$ with the actuator slot covered by thin tape and comparing it to the one with an open actuator slot as plotted in Fig. 9.2 (a\&b). It is assumed that the affected part in the impulse response is caused by sound generating one TS-wave at the disturbance generator (DG) and a second correlated TS-wave at the roughness of the actuator slot (A). Therefore the second TS-wave reaches the error sensor before the first one is detected at the reference sensor and this results in a 'non-causal' part in the impulse 


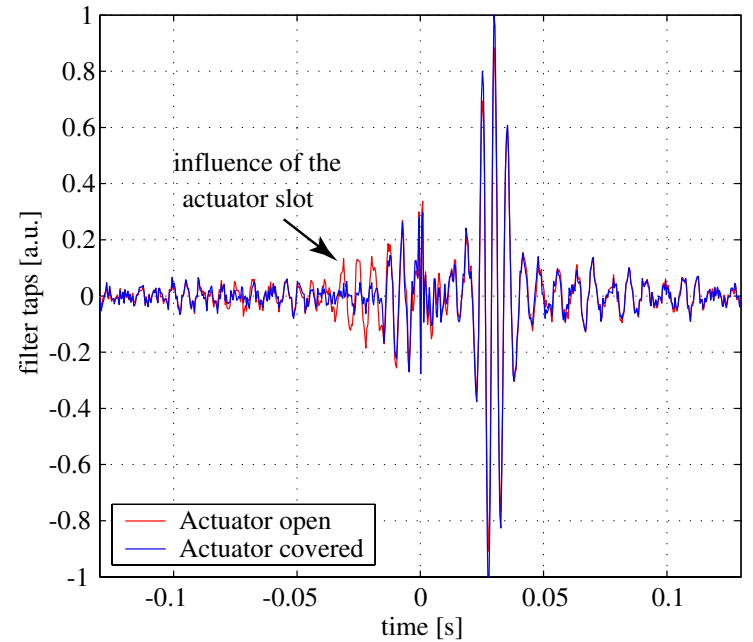

(a) The impulse response $H_{r e}$ between the reference and the error sensor with and without a covered actuator slot.

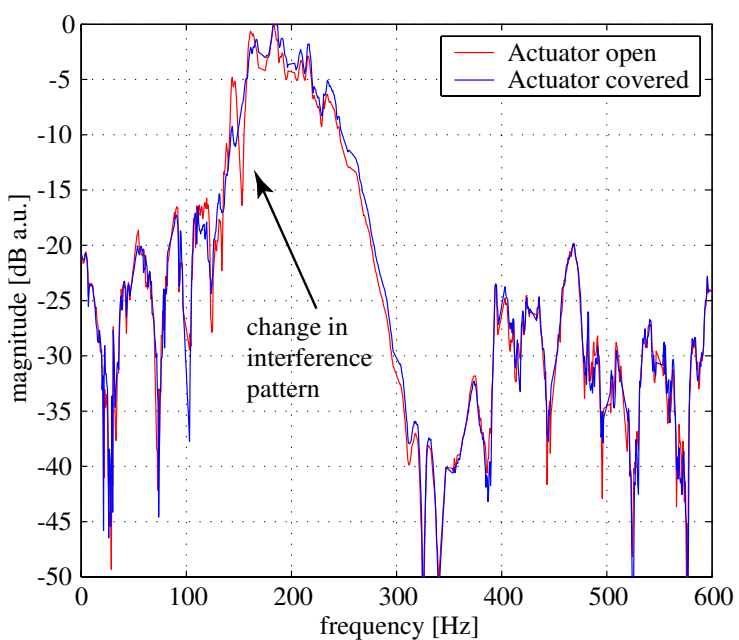

(b) The magnitude of the transfer function $H_{r e}$.

Figure 9.2: The influence of the roughness of the actuator slot on $H_{r e}$

response. This assumption is supported by the comparison of the travelling distances (see No. 3 in Tab. 9.1).

Further insight into the complex relation between the different signals can be gained by the analysis of the various spectra and correlations between the hot-wire sensors, the microphone and the accelerometer shown in Fig. 9.3 (a-e).

Fig. 9.3 (a) shows the PSD of the error sensor signal $S_{e e}$ with and without AWC as well as the theoretical optimum calculated from the coherence $C_{r e}$ between the reference and error signal. The latter is shown in Fig. 9.3 (b) for the cases with and without control ${ }^{1}$. $S_{e e}$ is reduced by up to $17 \mathrm{~dB}$ and the theoretical optimum matches the obtained $S_{e e}$ with AWC quite well with a few exceptions. The coherence is reduced by the AWC system in approximately the same frequency range, as expected. But there are obviously remainders in the frequency ranges $150-180 \mathrm{~Hz}$ and $300-400 \mathrm{~Hz}$ indicating a further reduction potential of the AWC system. A closer look at the Fig. 9.3 (a) reveals that the responsible disturbances are already at low level caused by the dispersion related damping. Furthermore they show a resonance pattern at $370 \mathrm{~Hz}$ revealing the influence of vibrations on the measured wall-shear-stress signals. This is supported by

\footnotetext{
${ }^{1}$ Qualitatively, the coherence matches the one obtained in the last chapter (see Fig. 8.3 (b)) thereby demonstrating that the slightly different experimental conditions in the present experiment did not fundamentally change the observed physics.
} 


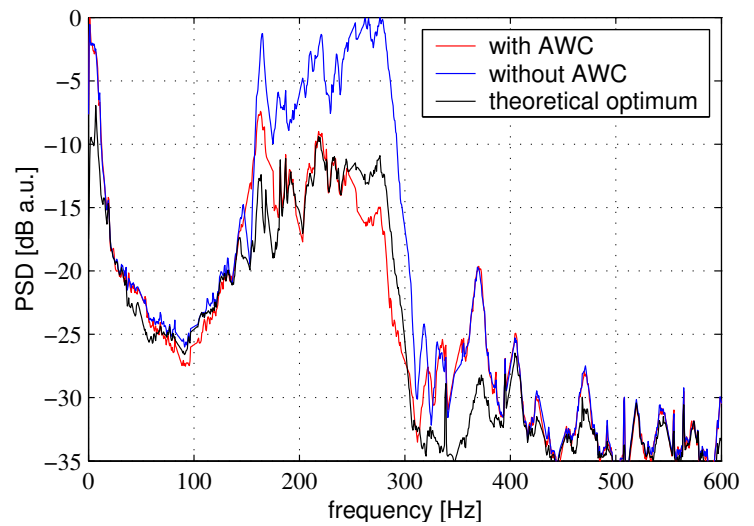

(a) The PSD of error sensor signal $\left(S_{e e}\right)$ with and without AWC and theoretical optimum calculated from the measured coherence.

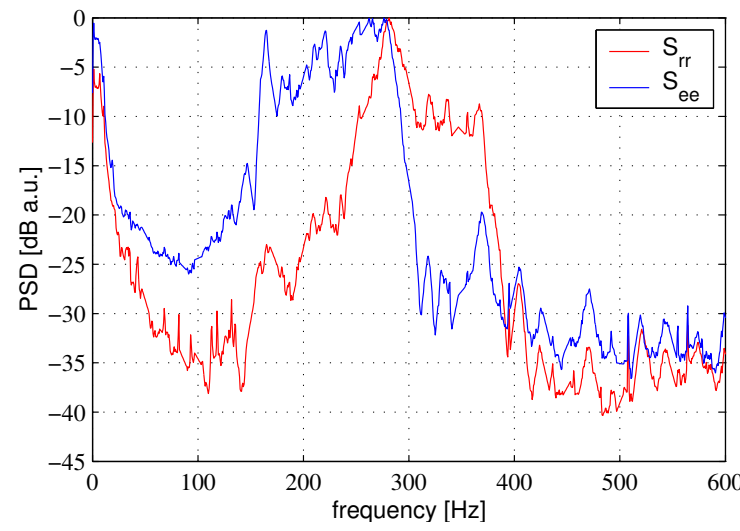

(c) The PSD of the reference $\left(S_{r r}\right)$ and the error $\left(S_{e e}\right)$ signal.



(b) The coherence between the reference and error sensor $C_{r e}$ with and without AWC .

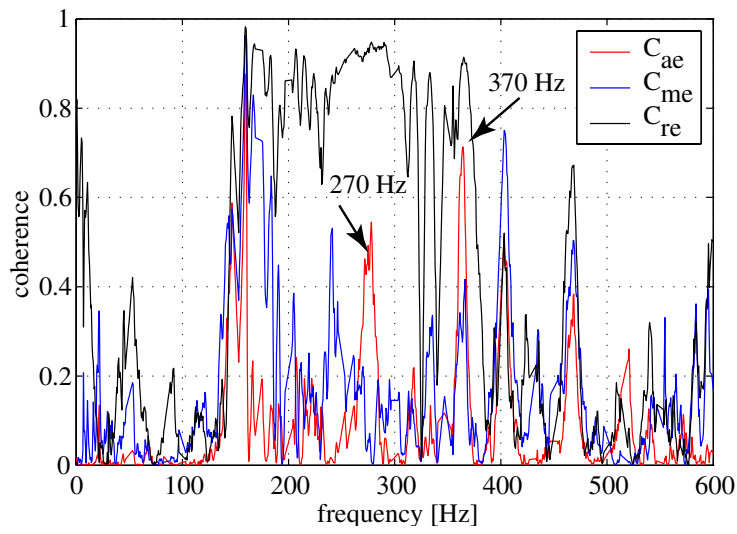

(d) The coherences between the mircophone $\left(C_{m e}\right)$ and the accelerometer $\left(C_{a e}\right)$ with the error sensor signal. For comparison the coherence between the reference and the error sensor $\left(C_{r e}\right)$ without AWC.

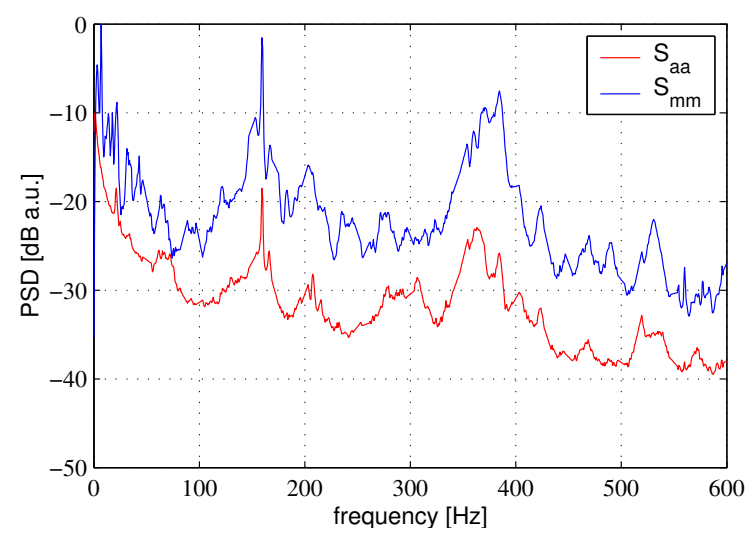

(e) The PSD of the microphone signal $\left(S_{m m}\right)$ and the accelerometer $\operatorname{signal}\left(S_{a a}\right)$.

Figure 9.3: Spectra and correlations for a SISO AWC system in a noisy environment. 
the coherence $C_{a e}$ between the error sensor and the accelerometer signal in Fig. 9.3 (d). Also presented in the same figure is the coherence $C_{m e}$ between the error sensor and the microphone indicating a small coherence of approximately 0.2 in the whole relevant spectrum. This may be the reason why some of the coherence $C_{r e}$ between the reference and the error signals cannot be eliminated by the AWC system (see Fig. 9.3 (b)). This is particularly the case in the frequency range $(150-180 \mathrm{~Hz})$ where the AWC system has only small effects on the spectrum of the error signal and the coherence remains as high as about 0.7. Hence, it can be concluded that this part is caused by sound generated TS-waves at the slit of the actuator. This also explains why the AWC system cannot effectively control the related disturbances which have been shown to be 'noncausal' from the point of view of the AWC system (see Fig. 9.2). Also explained by this fact is why the AWC does not reach the theoretical optimum at the mentioned frequency range. On the other hand the attained reduction near $270 \mathrm{~Hz}$ is even higher than the theoretically expected. This effect is caused by the streamwise degradation of the coherence by amplitude dependent nonlinear effects as mentioned previously. The high amplitude in this case is likely to be caused by a high level of excitation due to vibrations as indicated by the measured coherence $C_{a e}$ to the accelerometer signal.

Even though not all of the complex correlation between the presented data are completely understood there are some conclusions that can be summarized:

- Beside the TS-waves the wall-hot-wire sensors detect sound and vibrations of the plate.

- High correlation between TS-waves and sound is measured that is related to 'noncausal' components in the relevant transfer function. It turned out that the most prominent components of this correlation is due to the acoustic receptivity of the actuator.

- The actuator radiates sound while generating the counter-phase TS-wave that can generate TS-waves at upstream roughness and unevenness.

What are the consequences for the realizability of an active wave cancellation system under such conditions? How can theses effects limit the performance of the system?

\subsection{Acoustic feedback effects}

As discussed in the previous section a kind of acoustic feedback arises due to the sound that is radiated from the actuators while they excite the cancellation TS-waves (Opfer et al. 2002). Coherent TS-waves are generated by this means at roughness elements upstream of the actuators, and this can even destabilize the control system depending 


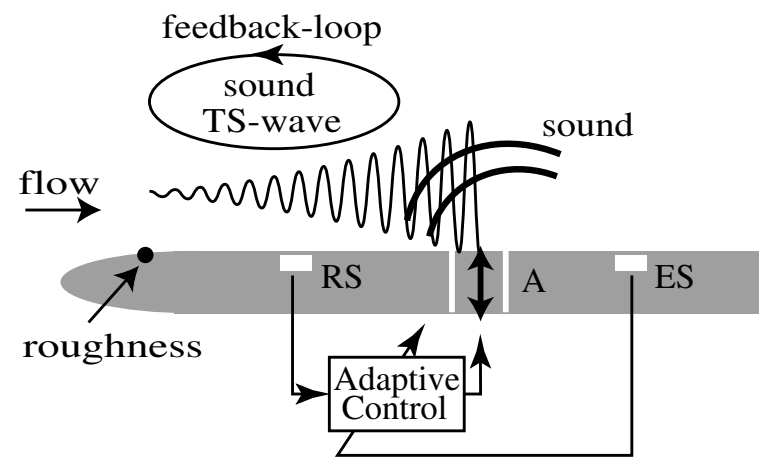

Figure 9.4: Schematic diagram of the acoustic feedback effect.

on the acoustic receptivity of the roughness and on the amplification of these waves while they propagate downstream. The feedback loop and the resulting destabilization of the control system is illustrated by Fig. $9.4^{2}$. The strength of the feedback has been decreased significantly by lowering the acoustic receptivity of the roughness due to covering the slot by thin tape. Increasing the positive streamwise pressure gradient led to an unstable control system caused by the higher amplification rates of the boundary layer.

\subsection{Sensitivity of the sensors to the sound particle velocity}

A second type of acoustic effects is caused by the sensitivity of the sensors to the sound particle velocity; this leads to a coherence of up to 0.7 between the hot-wire sensors and a microphone signal recorded far away from the wall. A whole of problems arises from this fact.

Assuming, e.g. that only the error sensors detect sound, the control system will minimize the error signal using both the excited TS-waves and the radiated sound field. This impairs the minimization of the amplitude of the remaining TS-waves.

Also non-causal parts in the measured transfer function between reference and error sensors can arise from correlated TS-waves that are generated at an upstream roughness and reach the reference sensor later than the sound signal that is detected at the error sensor.

\footnotetext{
${ }^{2}$ This effect is likely to be responsible for the observed destabilization of an AWC system in a different experimental set-up where the system was operated to cancel TS-waves in the boundary layer of a modified NACA 0008-profile. (private communication from D. Sturzebecher, TU-Berlin)
} 
Even more complicated become the effects if also the reference signals are contaminated by sound. Furthermore, there are TS-waves generated by acoustic pressure fluctuations at the slit of the actuator. These may reach the error sensor prior to the arrival at the reference sensor of the other correlated TS-waves that have been generated by the same acoustic events further upstream. This leads to non-causal parts in the relevant transfer function. The adapted filter taps of the control system show a kind of prediction behavior to cope with these non causal effects, but in general the performance of the system will be decreased.

In order to eliminate these effects attempts have been made to subtract the sound detected at the sensors digitally, by means of an adaptive filter modelling the transfer function between the microphone and the hot-wire sensor and therefore predicting the sound at the latter. However, possibly due to the relatively high correlation between the sound and the TS-waves generated mainly by the sound, it was not possible to attain a separation of the two parts. 


\section{Chapter 10}

\section{Influence of 3D effects on a nonlinear AWC.}

This chapter is devoted to effects that have been observed by Evert in an experimental study dealing with a nonlinear active wave cancellation system (Evert 2000).

Fig. 10.1 (a) shows a sketch of the experimental set-up, namely a SISO AWC system which consists of a reference sensor (RS), an actuator (A) and an error sensor (ES) built into a flat plate. Both sensors were wall hot-wire sensors of the same type as used in the present study.

In order to investigate nonlinear effects occurring in the propagation of TS-waves 'artificial' broadband 2D TS-waves were introduced into the boundary layer by blowing and suction through a spanwise slot, the disturbance generator (DG).

The experiments were carried out at a flow velocity $U_{\infty}=11 \mathrm{~m} / \mathrm{s}$ leading to the parameters that characterize the investigated Blasius boundary layer (given in the table in Fig. $10.1(\mathrm{~b}))$.

Besides the TS-waves expected from linear theory Evert observed subharmonic and harmonic parts in the measured spectra indicating nonlinearities in the propagation of the excited disturbances. Such components of the spectra can originate from quadratic frequency mixing generating frequency components at the difference and the sum of the primary frequencies. The degree of the occurring nonlinearity was governed by the amplitude of the disturbances and could therefore be chosen by the excitation amplitude. At very high excitation amplitudes such mixing products appeared also within the instability frequency range which may be explained by higher order nonlinearities of odd degree or by multiple nonlinear interactions.

In addition to a linear model based on adaptive filters Evert developed a nonlinear extension of the model based on Volterra-series. Thereby higher order correlations of 


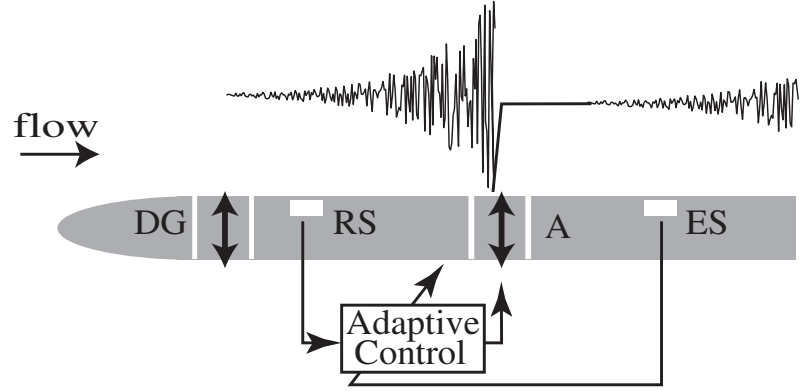

(a) The experimental set-up of the SISO AWC system.

\begin{tabular}{||c|c|c|c|c||}
\hline device & $\mathrm{x}[\mathrm{cm}]$ & $R e$ & $F_{\min }$ & $F_{\max }$ \\
\hline \hline DG & 18 & 332 & 168 & 238 \\
\hline RS & 26 & 409 & 107 & 196 \\
\hline A & 35.5 & 485 & 77 & 162 \\
\hline ES & 42 & 531 & 65 & 145 \\
\hline
\end{tabular}

(b) Boundary layer parameters: virtual leading edge distance, Reynolds number (based on the Blasius length), and lower and upper bounds of the region of amplification (non-dimensional frequency).

Figure 10.1: AWC of artificial broadband disturbances taking into account the occurring nonlinear effects.

the input signal were considered. For the implementation of the algorithm into a realtime controller it was necessary to reduce the computational cost. This was possible by the implementation of Kautz-filters which reduced the model order by making use of a priori knowledge, e.g. the band-pass character of the input signal. Thus, Evert was able to implement the algorithm in an adaptive nonlinear real-time controller. The degree of nonlinearity that was considered by the controller was limited to the order 2 by the real-time demand.

Fig. 10.2 (a) shows the results of the nonlinear wave cancellation experiment with a filter that could cope with quadratic nonlinearities. As expected, in addition to the reduction obtained with the linear part of the filter some further reduction in the spectrum of the error signal is achieved when the quadratic part of the filter is switched on.

Regarding the wall-normal profile of the flow velocity fluctuations in the boundary layer above the error sensor (Fig. $10.2(\mathrm{~b})$ ) it turns out however that the activation of the nonlinear part of the filter leads to an increase of the fluctuations rather than 
(a) PSD [dB rel. a.u.]

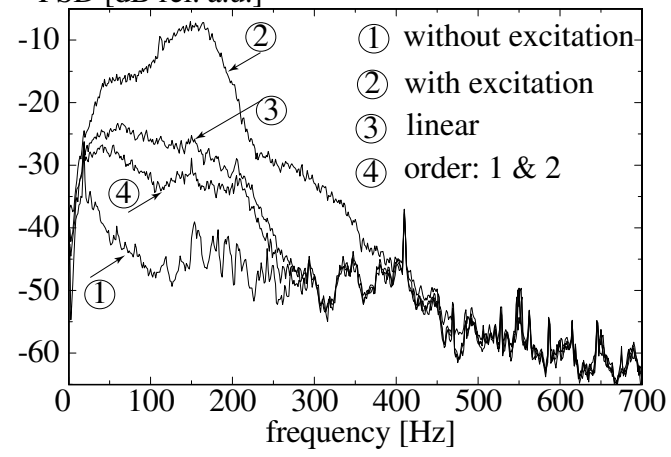

(b) $\mathrm{u}_{\mathrm{rms}} / \mathrm{U}[\%]$

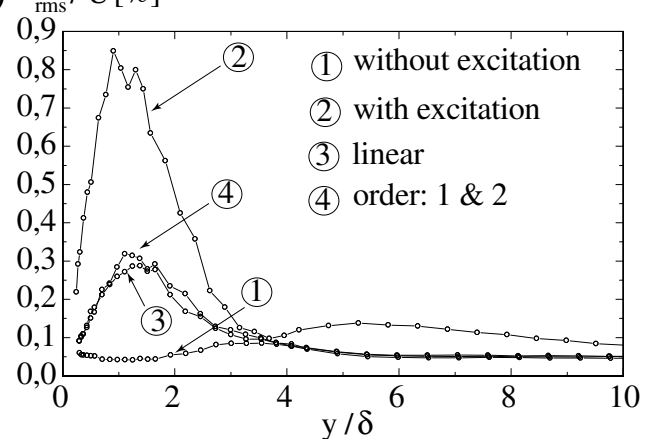

Figure 10.2: Nonlinear wave cancellation using an error signal detected at the wall. (from (Evert 2000))

to a further reduction. Remarkable is that the wall-normal profile of the remaining disturbances does not show the outer maximum as expected for 2D TS-waves.

Therefore a second experiment was performed with the error signal taken from a hotwire probe in the boundary layer at that distance from the wall where the fluctuations of the flow velocity are maximum. The result is shown in Fig. 10.3 (a) \& (b), and indeed, with this modified version of the AWC device a further reduction of the fluctuations in the inner boundary layer is achieved when the nonlinear part of the filter is activated. This is however connected with a significant increase of the fluctuations in the outer part of the boundary layer, in contrast with the result in Fig. 10.2 (b).

(a) PSD [dB rel. a.u.]

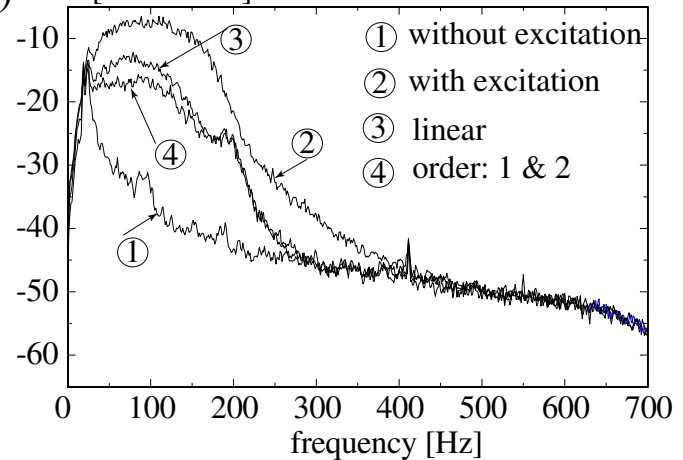

(b)

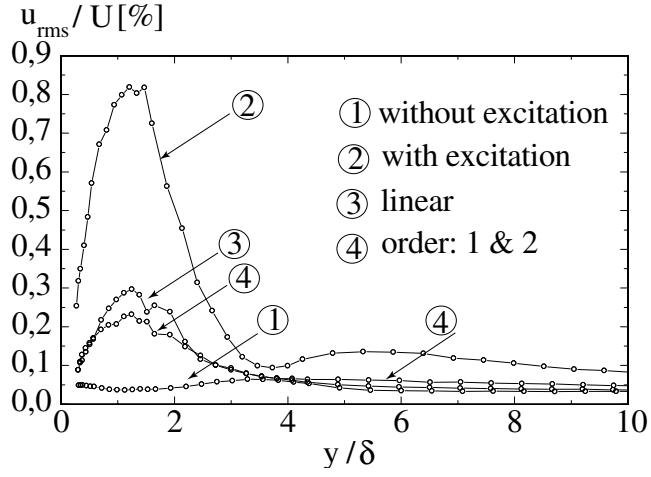

Figure 10.3: Nonlinear wave cancellation using an error signal detected in the boundary layer. (from Evert (2000)) 
The remaining disturbances that cannot be cancelled by the linear device but can be affected by the nonlinear device, have wall-normal profiles ((4) in Fig.10.2 (b) and Fig.10.3 (b), respectively) that differ between the two experiments.

What is the reason for this observation?

The stability diagram of the investigated boundary layer for $3 \mathrm{D}$ disturbances is plotted in Fig. 10.4 (a). The contour lines indicate the curves of zero amplification of the disturbances for the respective spanwise wavenumbers. The disturbance generator (DG) and the components of the cancellation device are marked to indicate the relevant region of the stability diagram.

Wall-normal profiles of the disturbance are presented in Fig. 10.4 (b) for different non-dimensional frequencies $F$. These have been computed for the Reynolds number $R e=531$ pertaining to the streamwise position of the error sensor. The frequencies are chosen according to the lower and the upper bound of the unstable region $(F=63.1$ and $F=141.8$, respectively). The third non-dimensional frequency $(F=93.5)$ corresponds to $120 \mathrm{~Hz}$ which was assumed to be a reasonable choice to represent a typical disturbance of the spectra and wall normal profiles in the figures 10.2 and 10.3. As expected, the main maximum of the profile is shifted away from the wall when the frequency is decreased, i.e. when the wavelength is increased. In addition, the outer maximum slightly increases with increasing frequency.

Fig. $10.4(\mathrm{c})$ presents wall normal profiles of the streamwise velocity component dependent on the dimensional spanwise wavenumber $\beta^{*}$. It becomes obvious that by increasing the inclination angle of the wave the main maximum also shifts away from the wall but additionally the outer maximum even disappears for very high spanwise wavenumbers as for $\beta^{*}=7.3 \mathrm{~cm}^{-1}$. The latter can be seen as an example profile that is not linearily unstable according to the stability diagram but may be generated by nonlinearities. Furthermore the gradient of such profiles directly at the wall is decreased by increasing the spanwise wavenumber. This gradient is proportional to the wall shear stress that is measured by the wall hot-wire sensors.

These properties of 3D disturbances may explain the results obtained by Evert.

When using the wall hot-wire sensor as error sensor detecting the wall shear stress 2D waves will cause higher signal components than 3D waves (Fig. 10.2 (a)). The shape of the profile of the remaining disturbances (Fig. $10.2(\mathrm{a})$ ) when linear control is applied resembles the one of $3 \mathrm{D}$ waves, i.e. missing the outer maximum, the inner maximum is slightly shifted away from the wall, and the velocity gradient at the wall becomes smaller.

These remaining disturbances are affected by the nonlinear control, resulting in a reduction of the wall shear stress but an increase in the measured velocity fluctuations. Therefore the 3D disturbances observed underlie a nonlinear process of propagation 


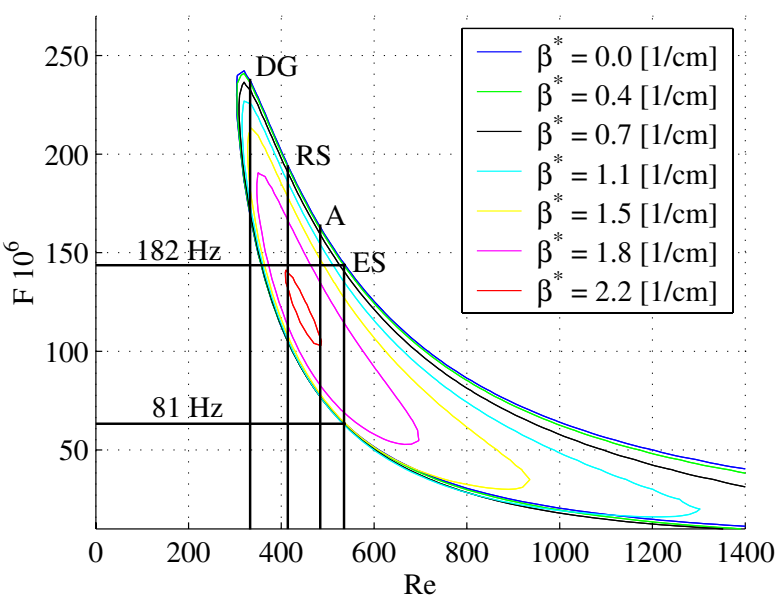

(a) The stability diagram of the investigated boundary layer for 2D and 3D TS-waves.

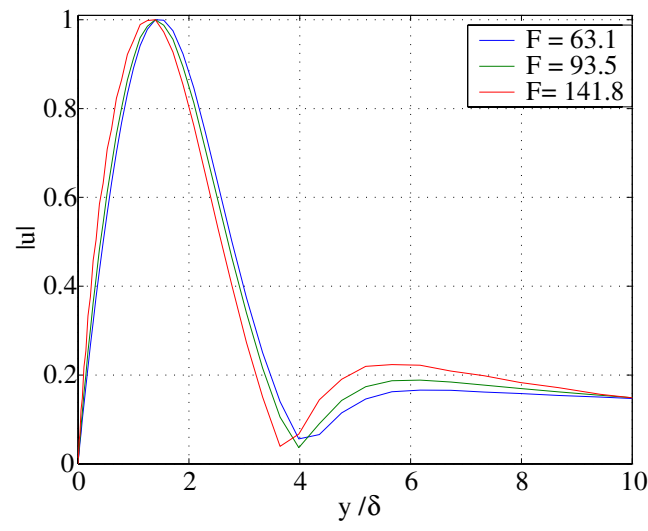

(b) Wall normal TS-profiles for different frequencies in the unstable region, $\beta^{*}=0 \mathrm{~cm}^{-1}$.

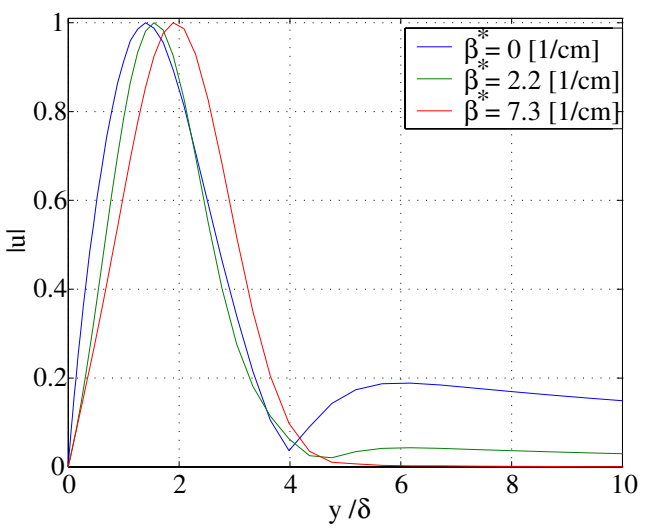

(c) Wall normal TS-profiles for different spanwise wavenumbers, $F=93.5$.

Figure 10.4: Linear stability characteristics of the investigated boundary layer concerning 3D disturbances. (obtained by a linear stability solver from A.V.Boiko described in Boiko (2000))

and/or their generation causes higher order correlation in the measured signals.

This connection between 3D disturbances and nonlinearities becomes even more obvious in the AWC experiment where the hot-wire probe in the boundary layer was 
used as error sensor(Fig. 10.3). Measuring the velocity, 3D components will result in stronger signal components than in the case of the wall hot-wire sensor measuring the wall shear stress.

When only linear control is applied the profile exhibits a stronger outer maximum as in Fig. 10.2 (b). Hence, it appears to be likely that the AWC (reducing the sum of all measured components in the signal) cancels besides the stronger 2D part also 3D components. Latter is possible only locally confined to the location of the error sensor.

On the other hand, if the wall hot-wire sensor is used as error sensor, higher amplitudes of the $2 \mathrm{D}$ cancellation waves are needed for the compensation of the detected nonlinear 3D-waves leading to a decrease of the inner maximum (as expected), however, to an increase of the outer maximum because of the unbalance between the 3D- and the $2 \mathrm{D}$ wave in the outer boundary layer. These 3D disturbances must have been generated by a nonlinear process because they cannot be influenced by the linear system.

The conclusion that can be drawn for the active wave cancellation in general is that due to the 3D disturbances that are generated by nonlinearities in the propagation of high amplitude 2D waves, it is necessary to consider both three-dimensionality and nonlinearity, if an efficient nonlinear AWC is to be implemented. For the development of suitable AWC devices and controllers that can cope with nonlinear effects it will be indispensable to distinguish between 2D and 3D disturbances to cope with nonlinear effects. The relation between quadratic frequency mixing and the occurring 3D effects needs some further investigation and may even lead to some general insight into the physics of transition and therefore also show ways to design a cancellation device dealing with $3 \mathrm{D}$ and nonlinear disturbances at the same time. 


\section{Chapter 11}

\section{Extension to the demands on an AWC system}

The demands to an AWC system, as described in chapter 3, have to be extended with regard to the experimental results obtained in this study. This chapter summarizes the findings influencing the demands, and some suggestions are made how to cope with the observed effects.

\subsection{Observability}

\subsubsection{Acoustic sensitivity of the sensors}

As explained previously the acoustic sensitivity of the sensors may lead to a number of complications concerning the AWC. Thus it is necessary to remove the acoustic part from the signal.

In general, this may be achieved by digital filtering. However, the attempts that have been made in the present study were not successful, probably because of the correlation between the sound and the TS-wave to be separated and the fact that there is no unambiguous relation between sound pressure and particle velocity.

A second approach may be to introduce a kind of wavenumber filtering by placing two sensors close to each other in streamwise direction. The preferred streamwise distance would match half of the streamwise TS-wavenumber and thus the sound component could be reduced by subtraction of both signals. The resulting difference signal would then be used as an input of the controller. In addition, this technique can be used to obtain a noise reduction in the signals when placing the sensors in a distance of approximately half the expected TS-wavelength. 


\subsubsection{Wall shear stress dependence of the modes}

The wall shear stress signals contain components of all modes present in the disturbance wave field, which may have significantly different wall normal eigenfunctions, resulting also in different wall shear stress components. The task the AWC system has to manage is to modally decompose the wave field in order to generate the appropriate counterphase modes that also reduce the velocity fluctuation in the boundary layer.

It became clear from the interpretation of the results of Evert (2000) that the AWC system controls the fluctuations of the wall shear stress and not of the velocity fluctuations directly. This may lead to a kind of local control that affects only the close surrounding of the error sensor.

The investigations dealing with a 3D AWC system exposed to point-source disturbances, containing many different modes, showed that the system was able to control the velocity fluctuations of the boundary layer in a whole range of spanwise wavenumbers. Thereby the system showed that it could decompose the wave field into a small number of modes which were treated differently.

This is useful for nonlinear cancellation devices as pointed out in the previous chapter.

\subsection{Controllability}

The experiences made in the development of actuators that are suitable for an AWC system have been discussed in detail in chapter 6 . This section summarizes the main conclusions.

Crucial to the task of developing a suitable actuator is the demand that the generated spanwise wavenumber spectrum has to be sufficiently sampled by the error sensor array.

The spanwise wavenumber spectrum of the Type I actuator clearly indicated that one cannot rely on the spatial filtering properties of the boundary layer. The high spanwise wavenumber components generated by this actuator propagate to the streamwise location of the error sensors. Thus the actuators have to generate a suitable wavenumber spectrum in the first place. If this cannot be achieved by constructional measures, the spatial filtering approach by a combined driving of neighboring actuators may become an alternative, to some extent, as previously discussed (section 6.2).

Furthermore the sound generation of the actuator has to be minimized to reduce the acoustically induced feedback. This may be achieved by using a kind of dipole actuator, where the fluid is sucked in through one slot while the fluid is blown out by a second slot, thus acting as an acoustic dipole. 
In order to minimize the effect of 'non-causal' parts in the relevant transfer functions that lead to uncontrollable disturbances, it is necessary to reduce the acoustic receptivity of the actuator to external perturbations. One way to achieve this, may be to use membrane actuators of the kind as Sturzebecher and Nitsche (2002) suggested. 


\section{Chapter 12}

\section{Summary, conclusions, and outlook}

The present study deals with the design of an active wave cancellation system to simultaneously cancel 2D and 3D Tollmien-Schlichting waves in order to delay the laminar-turbulent transition.

Tollmien-Schlichting waves may originate from a variety of different excitation processes, e.g. by scattering of long-wave-length sound waves at roughness elements or other non-uniformities of the wall surface, or by short-wavelength pressure fluctuations due to free-stream turbulence. Such 'natural' TS waves have been investigated in an flat plate boundary layer with the objective to cancel the resulting wave field by superposition of an artificially generated 'counter-phase' wave field.

To account for spanwise variations in amplitude and phase the wave field is spatially and temporarily sampled by a spanwise array of reference sensors in order to compute an appropriate set of signals that drive the spanwise array of actuators further downstream. For this purpose, essentially, the transfer matrix containing all the transfer functions between pairs of reference sensors and actuators has to be evaluated in order to simulate the physical process of propagation, including the dispersion relation which depends on the frequency, the Reynolds number, and the spanwise wavenumber. The modelling of the transfer matrix was implemented by means of a multiple-inputmultiple-output adaptive digital filter. The taps of the filter were adjusted in a least mean square sense to minimize the overall power at an array of error sensors that is placed downstream of the actuators. This has been implemented in real-time by means of a multiple-input-multiple-output LMS algorithm.

\section{The demands on an active wave cancellation device}

At first the demands to such a system coping with 3D disturbances have been workedout with the objective to design a suitable device for wind tunnel investigations. 
The lateral correlation of the disturbances depends on their origin as well as on their lateral spreading as they propagate downstream to the location of the cancellation device. The maximum number of degrees of freedom contained in the wave field is given by the product of the maximum spanwise wavenumber of the disturbance spectrum and its maximum spanwise correlation length and such determines the maximum number of degrees of freedom the control system must be able to model. By clustering modes that propagate similarly this number may be further reduced. The Nyquist theorem assures the complete discrete representation and in this way determines the minimum lateral spacing of the sensors and actuators; and furthermore, the maximum wavenumber component the actuators are tolerated to generate; in other words the spanwise distribution of the generated cancellation wave must be sufficiently smooth.

In order to model the transfer process of the piece of boundary layer between the reference sensor array and the actuator array, Wiener filter theory has been applied to find the optimum transfer matrix. It turned out that this matrix is degenerating to a single transfer function, if the streamwise distance between the reference sensors and the actuators tends to zero. However, due to the random character, the constraint of causality applies to the control system, requiring a minimum streamwise distance between these components. Thus, an alternative way to simplify the modelling of the propagation process was found to be the introduction of an additional spatial filtering, e.g. by the design of actuators. If this filtering contains the same dependence on the spanwise wavenumber as the dispersion relation of the boundary layer, again only a single transfer function is needed for the whole modelling. In case these strategies are not followed, one may exploit the Toeplitz-form of the relevant transfer and correlation matrices in order to simplify, not only the temporal inversion, but also the spatial inversion needed to obtain the optimum filter matrix.

\section{The design of the cancellation devices}

The spanwise distance between the sensors was chosen according to the lateral correlation of the 'natural' disturbances measured in a prior study. A slot actuator was designed and constructed that could be incorporated into the flat plate. Small loudspeakers in narrow enclosures have been used as actuators providing wall-normal suction and blowing through a $0.3 \mathrm{~mm}$ spanwise slot. In contrast to the pressure chamber-tube actuators used in prior studies this type of actuator generated a flat frequency spectrum of wall-normal velocity fluctuations at sufficiently large amplitudes. For the purpose of smoothing the spanwise excitation distribution, the neighboring actuators generated disturbances in a combined slot. In order to achieve the necessary signal-processing in real-time the existing hardware had to be upgraded and partially renewed. Similarly, the basic DSP software was adapted and the used algorithms were implemented in real-time versions. 
However, first experiments carried out with the designed experimental set-up showed that the design precautions were inadequate to assure a smooth spanwise disturbance distribution of the actuators. Measurements concerning the streamwise propagation of the excited disturbances showed that the spatial filtering properties of the boundary layer itself were too weak to compensate for the generated high wavenumber components. The associated small spanwise spreading of the disturbances constricted the demand of controllability, meaning that the neighboring error sensors were not affected much by the actuator. Furthermore, the streamwise span of the cancellation device turned out to be too large leading to a high degradation of the coherence which is essential for the success of the linear methods applied. Additionally, it turned out that a $2 \mathrm{D}$ roughness caused by the slot of a former disturbance generator was responsible for the main part of the natural disturbance wave field, which therefore was predominated by $2 \mathrm{D}$ disturbances. First active wave cancellation experiments were carried out with this device considering only streamwise correlations, because the restricted spanwise spreading of the cancellation disturbances did not allow to consider adequately lateral correlations, too. However, the spanwise distribution of the remaining disturbance wave field also indicated insufficient spatial sampling.

Based on these results a second cancellation device has been developed. The wavenumber spectrum of the actuator has been optimized in the design and improved constructionally. It could also be shown experimentally that tailoring the relation of the driving signals of neighboring actuators can result in a pseudo actuator, which generates a smoother disturbance distribution and thereby a wavenumber spectrum confined to the Nyquist wavenumber range. Therefore such pseudo actuator may become an option to the constructional design that can meet the demands of a specific boundary layer. To improve the spatial sampling of the disturbance wave field the spanwise distance of the sensors and the actuators was halved. This approach was limited by the size of the new actuators. Furthermore the streamwise span of the cancellation device has been shrunk in order to obtain a higher streamwise correlation. To avoid the predominance of $2 \mathrm{D}$ disturbances in the investigated wave field the mentioned 2D acoustic roughness has been minimized by covering the slots of the former disturbance generators.

It is of great practical interest in general and in the following experiments, to what extent such an active wave cancellation system has to distinguish between the different oblique wavenumber components of the disturbance wave field. Thereby the angle dependence of the two transfer functions between the reference sensor array and the actuator array comes into play, namely the transfer function for the boundary layer and the transfer function for the active wave cancellation system. In fact, if these two transfer functions exhibit the same dependence on the spanwise wavenumber, there is no need for the AWC system to distinguish between the wavenumber components. This is also true in the case that the disturbance field consists only of a single wave number 
component. Otherwise the off-diagonal elements of the mentioned transfer matrix have to be considered to treat the spanwise Fourier components differently. In any case the diagonal elements, which are the streamwise transfer functions, are the most important components of the cancellation system.

\section{Cancellation of natural TS-waves}

The improved cancellation device has been applied to natural TS-waves in two control modi. In the first case only the streamwise transfer functions have been considered, namely the diagonal elements of the transfer matrix designated as streamwise independent channels. In the second case the complete $4 \times 4$ matrix has been used to generate the cancellation wave field denoted as coupled channels. The measured velocity fluctuations in the boundary layer downstream of the error sensors were reduced by up to $22 \mathrm{~dB}$ for the strongest component in the spectrum. The lateral RMS-distribution in the relevant frequency range shows a reduction by up to $18 \mathrm{~dB}$. No significant improvement could be achieved by the addition of the off-diagonal elements of the transfer matrix to the controlling algorithm.

Two further experiments, namely the study of the TS wave field that was excited by a point-source, and the study of the acoustic receptivity of the boundary layer, provided an explanation why the performance of the cancellation device is not improved - under the given test conditions - by the coupling of the channels.

\section{Point source experiment and acoustic receptivity}

Unsteady suction and blowing through a short narrow slit served as a TS point source. The excited velocity fluctuations were measured by a hot-wire probe at various lateral and streamwise positions. Only the fluctuations correlated with the signal of the source were considered in the further analysis. The lateral wavenumber spectra show that oblique waves propagating within the streamwise span of the control system could not be sufficiently sampled.

A similar experiment has been performed with a loudspeaker in the settling chamber of the wind tunnel exciting the 3D waves to be investigated. It turned out that the obtained spanwise wavenumber spectrum had the same shape as in the point-source experiment, except for a strong zero-wavenumber peak that is superimposed on this spectrum. While the broadband part of the spectrum is caused by the acoustic receptivity of the point source, which is driven by the external sound pressure, the strong $2 \mathrm{D}$ component is generated at the a $0.1 \mathrm{~mm}$ thick technical roughness remaining from covering the former disturbance generators. 
Similarly, the natural disturbances which are supposed to originate mainly from the acoustical noise in the wind tunnel have a strong zero-wavenumber component. The damping of this $2 \mathrm{D}$ part cannot be improved by considering the off-diagonal elements in the transfer matrix. The broadband part of the spectrum aimed to be reduced by the system was found to be $12 \mathrm{~dB}$ below this $2 \mathrm{D}$ part and extends far beyond the Nyquist wavenumber of the system. Thus, an effective cancellation cannot be demonstrated, even if the full 3D capacity of the system is utilized.

\section{Cancellation of broadband point source TS-waves}

In order to gain a deeper insight into the effects associated with the consideration of the full transfer matrix, another cancellation experiment was carried out where the disturbances to be cancelled are excited by the point source. Again, the system has been operated in both control modi and the results were compared to the uncontrolled case. The performance has been evaluated by the spatial transfer function across the cancellation device, namely between the excitation signal and a movable hot-wire probe measuring the velocity fluctuations downstream of the error sensor array at different spanwise positions. Operating only the streamwise channels resulted in the reduction of a single wavenumber component in the spectrum by up to $25 \mathrm{~dB}$, whereas by the coupling of the channels only $17 \mathrm{~dB}$ of damping are achieved. However, by considering the complete transfer matrix the affected part of the spectrum is extended to the Nyquist wavenumber of the system. The difference in maximum reduction may be due to insufficient sampling, or caused by dispersing the cancellation power of the system over the whole spectrum. In any case the total power of the remaining disturbances as seen by the error sensors is decreased by the full 3D capacity of the system. Additionally, it turned out that the system operated by independent streamwise channels could not distinguish between 3D and 2D disturbances. Therefore a $2 \mathrm{D}$ cancellation wave was generated to cancel the primary $3 \mathrm{D}$ disturbance locally at the error sensor, namely the wall-hot-wire sensor, which resulted in a disturbance enhancement in the vicinity. In this context, it turned out that the dependence of the wall-normal profile of the streamwise velocity on the spanwise wavenumber needs also to be considered. Due to the decreased wall-shear stress in 3D waves the system will underestimate the amplitude of 3D waves leading to a systematic error in the reconstructed wave field.

\section{Acoustic and vibrational influences}

Furthermore a number of acoustic effects have been discovered impairing the action of the cancellation device.

A kind of acoustic feedback arises due to sound that is radiated from the actuators 
while exciting the cancellation TS-waves. Coherent TS-waves may be generated in this way at roughness elements upstream of the actuators, and this can destabilize the control system depending on the acoustic receptivity of the roughness and the amplification of these waves while they propagate downstream. In an extreme case, this may even lead to global instability.

A second type of acoustic effects is caused by the sensitivity of the sensors to the sound particle velocity; this leads to a coherence of up to 0.7 between the hot-wire sensors and a microphone signal recorded far away from the wall.

A number of severe problems arise from this fact. Assuming, e.g. that only the error sensors detect sound, the control system will minimize the error signal weighting both the excited TS-waves and the radiated sound field. This impairs the minimization of the amplitude of the remaining TS-waves. It turned out that also non-causal parts in the measured transfer function between reference and error sensors can arise from the correlated TS-waves that are generated at upstream roughness and reach the reference sensor later than the sound signal that is detected at the error sensor.

The effects become even more complicated if the reference signals are also contaminated by sound. Furthermore, it turned out that the acoustic pressure fluctuations may excite TS-waves at the actuator slit. These waves may reach the error sensors before the arrival of some other correlated TS-waves at the reference sensors which originate from a sound-roughness interaction further upstream.

The adaptive filter shows a kind of prediction behavior to cope with these non-causal effects, but in general the performance of the system is decreased.

In order to eliminate these effects attempts have been made to subtract the sound detected at the sensors digitally, by means of the adaptive filter modelling the transfer function between the microphone and the hot-wire sensor and therefore predicting the sound at the latter. Due to the relatively high correlation between the sound and the TS-waves generated mainly by sound it was not possible however to attain a separation of the parts in the hot-wire sensor signal.

\section{Influence of 3D effects on a nonlinear AWC}

The experimental results obtained by Evert (2000) who investigated a nonlinear AWC system, have been interpreted with respect to the observed disturbance remainders after control. The disturbances that could have been affected by the nonlinear AWC system showed a different wall-normal profile of velocity fluctuations compared to 2D TSwaves. Due to the similarity of the remaining disturbance profiles to the one of 3D disturbances the experimental observations made by Evert could be interpreted such that $3 \mathrm{D}$ disturbances may have been generated by nonlinearities in the propagation of high 
amplitude 2D disturbances. Therefore the resulting sensor signals could be related to each other by quadratic order correlation. Thus, the local reduction of the considered fluctuations (locally at the operated error sensor) can be explained by the cancellation of theses nonlinear 3D waves by artificial 2D-waves. This explains also the resulting changes in the wall normal profile depending on the location of the error sensor used in the control.

The conclusion that can be drawn for the active wave cancellation in general is that due to the 3D disturbances that are generated by nonlinearities, it is necessary to consider both effects at the same time in order to implement an efficient nonlinear AWC. For the construction of a suitable AWC device and controller, it will probably be inescapable to discriminate between $2 \mathrm{D}$ and $3 \mathrm{D}$ disturbances, in order to cope with these nonlinear effects.

\section{Outlook}

With respect to the complexity of the interactions between physical effects observed in this study, computer simulations are the choice to isolate the different influences. Thereby the strength of the discovered influences may be determined closer, and ways to cope with the resulting problems may be discovered.

Regarding the streamwise development of wave-packets, the computer simulations may lead to some further insight into the physics of transition to expand the method presented in this study with respect to the consideration of higher order correlations. Thus, the spatial structure of the correlation pulse could be traced into the highly nonlinear stages of transition, monitoring the evolution of temporal and spatial spectra. The resulting knowledge may give important hints, how to construct a future AWC system dealing with 3D disturbances of nonlinear origin, and may also explain the relation between quadratic frequency mixing and the occurring $3 \mathrm{D}$ effects mentioned in this study. 


\section{Bibliography}

Alexander, S. (1986). Adaptive Signal Processing. New York: Springer.

Baumann, M. (1999). Aktive Dämpfung von Tollmien-Schlichting Wellen in einer Flügelgrenzschicht., Volume 7:372 of Fortschr.-Bericht. VDI-Verlag. (in German).

Bendat, J. (1978). Statistical errors in measurement of coherence functions and input/output quantities. J. of Sound and Vibration 59(3), 405-421.

Bertolotti, F. (1991). Linear and nonlinear stability of boundary layers with streamwise varying properties. Phd thesis, The Ohio State Univ., USA.

Boiko, A. (2000). Receptivity of boundary layers to free stream axial vortices. Technical Report IB-223-2000 A10, German Aerospace Center (DLR), Institute of Fluid Mechanics, Göttingen.

Boiko, A., G. Grek, A. Dovgal, and V. Kozlov (2002). Origin of Turbulence in NearWall Flows. Springer-Verlag.

Burgess, J. (1981). Active adaptive sound control in a duct: A computer simulation. J. Acoust. Soc. Am. 70, 715-726.

Bushnell, D. (1990). Viscous Drag Reduction in Boundary Layers, Volume 123 of Progress in Astronautics and Aeronautics, AIAA Introduction. Bushnell, D.M. ; Hefner J.N.

Cousteix, J. (1992). Basic concepts on boundary layers, special course on skin friction drag reduction. Technical Report R-786, AGARD.

Eckelmann, H. (1997). Einführung in die Strömungsmeßtechnik. LAMM. Stuttgart: Teubner. (in German).

Elliott, S., C. Boucher, and P. Nelson (1992, May). The behaviour of a multiple channel active control system. IEEE Transactions on Signal Processing 40(5), 1041-1052.

Elliott, S., I. Stothers, and P. Nelson (1987, October). Multiple error LMS algorithm and its application to the active control of sound and vibration. IEEE Transactions on Acoustics, Speech and Signal Processing ASSP-35(10), 1423-1434. 
Evert, F. (2000). Experimentelle Untersuchungen zur dynamischen Beeinflussung einer Plattengrenzschicht. Ph. D. thesis, Math.-Nat. Fak. Univ. Göttingen. (in German).

Evert, F., D. Ronneberger, and F.-R. Grosche (1999). Dynamische Stabilisierung einer laminaren Plattengrenzschicht. ZAMM 79(Suppl.2), 603-604. (in German).

Evert, F., D. Ronneberger, and F.-R. Grosche (2000). Application of linear and nonlinear adaptive filters for the compensation of disturbances in the laminar boundary layer. ZAMM 80 (Suppl.1), 85-88.

Fischer, M. (1994). Untersuchung künstlich angeregter Instabilitäten in einer zweidimensionalen laminaren Grenzschichtströmung mit Hilfe der Particle Image Velocimetry. DLR-FB 93-58, DLR. (in German).

Gaster, M. (1975, Apr.). A theoretical model of a wave packet in the boundary layer on flat plate. Proc. R. Soc. Lond. 347(3), 271-289.

Gaster, M. and I. Grant (1975, Apr.). An experimental investigation of the formation and development of a wave packet in a boundary layer. Proc. R. Soc. Lond. 347(3), 253-269.

Gilyov, V. and V. Kozlov (1984). Excitation of Tollmien-Schlichting waves in the boundary layer on a vibrating surface. J. App. Tech. Mech. Phys. 25, 874-877.

Gmelin, C. (2001). Numerische Untersuchungen zur aktiven Dämpfung von Störungen im Vorfeld der laminar/turbulenten Transition. Ph. D. thesis, Institut für Aero- und Gasdynamik, Universität Stuttgart. (in German).

Haykin, S. (1996). Adaptive Filter Theory (Third edition ed.). Prentice Hall Information And System Sciences Series. Prentice Hall.

Henke, R. (1999). "A 320 HLF Fin" Flight Tests Completed. Air \& Space Europe $1(2), 76-79$.

Ivanov, A., Y. Kachanov, T. Obolentseva, and A. Michalke (1998). Receptivity of the Blasius-boundary-layer to surface vibrations. Comparison of theory and experiment. In Proc. of Int. Conf. on Meth. of Aerophys. Research, pp. 93-98. Inst. Theor. Appl. Mech. Novosibirsk.

Ivanov, A., W. Würz, S. Herr, S. Wagner, and Y. Kachanov (2001, April, 2-4). Experimental Investigation of $3 D$ Acoustic Receptivity due to a Surface Vibration on an Airfoil. Boundary-Layer Transition in Aerodynamics, EUROMECH Colloquium 423. Universität Stuttgart, Germany, Institut für Aerodynamik und Gasdynamik: Chairmen: S. Wagner, M. Kloker, U. Rist.

Joslin, R. (1998). Aircraft laminar flow control. Annu. Rev. Fluid Mech. 30, 1-29. 
Kachanov, Y. (1995). Three-dimensional instabilities in boundary layers. In J. v. I. R.A.W.M. Henkes (Ed.), Transitional Boundary Layers in Aeronautics, pp. 5570. Royal Netherlands Academy of Arts and Sciences.

Kachanov, Y. and A. Michalke (1994). Three-dimensional instability of flat-plate boundary layers: Theory and experiment. Eur. J. Mech., B/Fluids. 13, 401-422.

Kachanov, Y. and T. Obolentseva (1996). Development of three-dimensional disturbances in the Blasius boundary layer. 1. Wave-trains. Thermophysics and Aeromechanics 3(3), 225-243.

Kerschen, E., M. Choudhari, and R. e. a. Heinrich (1990). Generation of boundary layer instability waves by acoustic and vortical free-stream disturbances. In R. Arnal (Ed.), Laminar-Turbulent Transition, IUTAM Symp. 1989, pp. 477-488. Springer-Verlag Berlin-Heidelberg.

Klebanoff, P., K. Tidstrom, and L. Sargent (1962). The three-dimensional nature of boundary-layer transition. J. Fluid Mech. 12, 1-34.

Konzelmann, U. (1990). Numerische Untersuchungen zur räumlichen Entwicklung dreidimensionaler Wellenpakete in einer Plattengrenzschicht. Ph. D. thesis, Institut A für Mechanik der Universität Stuttgart. (in German).

Kuo, S. and D. Morgan (1996). Active Noise Control Systems - Algorithms and DSP Implementations. Wiley Series in Telecommunications and Signal Processing. Canada: JON WILEY \& SONS INC.

Ladd, D. (1990). Control of natural laminar instability waves on an axissymetric body. AIAA Journal 28(2), 367-369.

Ladd, D. and Hendricks E.W. (1988). Active control of 2-D instability waves on an axisymmetric body. Experiments in Fluids 6, 69-70.

Liepmann, H. and D. Nosenchuck (1982). Active control of laminar-turbulent transition. Journal of Fluid Mechanics 118, 201-204.

Liepmann, H. and G. Nosenchuk (1982). Control of laminar-instability waves using a new technique. J. Fluid Mech. 118, 187-200.

Mack, L. (1984). Boundary layer stability theory. Technical Report 709, AGARD. pp.3.1-3.81.

Milling, R. (1981). Tollmien-Schlichting Wave Cancellation. Phys. Fluids 24(5), 979981.

Morkovin, M. (1968). Critical evaluation of transition from laminar to turbulent shear layer with emphasis of hypersonical travelling bodies. Tech. Rep. 68-149, AFFDL. 
Neemann, K., S. Bake, A. Ivanov, and Y. Kachanov (2001, April, 2-4). Receptivity of Boundary Layers to 3D Wall Vibrations: Theory and Experiment. BoundaryLayer Transition in Aerodynamics, EUROMECH Colloquium 423. Universität Stuttgart, Germany, Institut für Aerodynamik und Gasdynamik: Chairmen: S. Wagner, M. Kloker, U. Rist.

Nelson, P. (1994). Active control of acoustic fields and the reproduction of sound. Journal of Sound and Vibration 177(4), 447-477.

Opfer, H. and D. Guicking (1999). Multichannel active noise control using in a rectangular duct using stabilized fast transversal filters. In Proceedings of the Sixth International Congress on Sound and Vibration, 5-8 July, Kopenhagen, Denmark, pp. 1597-1603.

Opfer, H., D. Ronneberger, and F.-R. Grosche (2002, July). Acoustic influence on the active control of 3D disturbances in a 2D boundary layer. Proc. of ASME FEDSM'02, No.31042, Montreal, Canada.

Oppenheim, A. and R. Schafer (1999). Discrete-time signal processing (Second edition ed.). Prentice Hall Information And System Sciences Series. Prentice Hall.

Pupator, P. and W. Saric (1989). Control of random disturbances in a boundary layer. AIAA Journal 89.

Raguse, A. (1998). Experimentelle Untersuchungen zur aktiven dynamischen Beeinflussung des laminar-turbulenten Umschlags in einer Plattengrenzschicht. Ph. D. thesis, Math.-Nat. Fak. Univ. Göttingen. (in German).

Schilz, W. (1965). Experimentelle Untersuchungen zur akustischen Beeinflussung der Strömungsgrenzschicht in Luft. Acustica 16(4), 208-223. (in German).

Schlichting, H. (1933). Zur Entstehung der Turbulenz bei der Plattenströmung. ZAMM 13, 171-174. (in German).

Schubauer, G. and H. Skramstad (1948). Laminar-boundary-layer oscillations and transition on a flat plate. Technical Report 909, National Advisory Comittee for Aeronautics.

Slock, D. and T. Kailath (1991). Numerically stable fast transversal filter of recursive least squares adaptive filtering. IEEE Transactions on Signal Processing 39(1), $92-114$.

Snyder, S. and C. Hansen (1994, Apr.). The effect of transfer function estimation errors on the filtered-x lms algorithm. IEEE Trans. Signal Processing 42, 950953.

Squire, H. (1933). On the stability of three-dimensional disturbances of viscous fluid flow between parallel walls. Proc. Roy. Soc. 142, 621-628. 
Stemmer, C. (2001). Direct Numerical Simulation of Harmonic Point Source Disturbances in an Airfoil Boundary Layer with Adverse Pressure Gradient. Ph. D. thesis, IAG, Fakultät für Luft- und Raumfahrt der Universität Stuttgart. Shaker Verlag.

Sturzebecher, D. and W. Nitsche (2002). Active Control of Tollmien-Schlichting Instabilities by Multi-Channel Sensor Actuator Systems, Volume 77 of NNFM, Contributions to the 12th STAB-Symposium, Stuttgart, Germany. Springer, Germany: Wagner, S.;Rist, U.;Heinemann, H.J.;Hilbig, R.

Sundaram, S., F.-R. Grosche, and H.-P. Kreplin (1997). Cross-correlation measurements in naturally occuring Tollmien-Schlichting waves. IB 223-97 A 37, DLR.

Thomas, A. (1983). The control of boundary-layer transition using a wavesuperposition principle. Journal of Fluid Mechanics 137, 233-250.

Thomas, A. (1990). Viscous Drag Reduction in Boundary Layers, Volume 123 of Progress in Astronautics and Aeronautics, AIAA Active wave control of boundary layer transition. Bushnell, D.M. ; Hefner J.N.

Tollmien, W. (1929). Über die Entstehung der Turbulenz. 1. Mitteilung, Nachr. Ges. Wiss. Göttingen, Math. Phys. Klasse 21-24. (in German).

Wehrmann, O. (1965). Tollmien-Schlichting waves under the influence of a flexible wall. Physi. Fluids 8, 1389-1390.

Widrow, B. (1970). Adaptive filters. In R. Kalman and N. DeClaris (Eds.), Aspects of Network and Systems Theory, pp. 563-587. New York: Holt, Rinehart and Winston.

Widrow, B., D. Shur, and S. Shaffer (1981). On adaptive inverse control. In Proc. of 15th Asilomar Conf., pp. 185-189.

Widrow, B. and S. Streans (1985). Adaptive Signal Processing. Engelwood Cliffs, N.Y.: Prentice Hall.

Wiegel, M. (1997). Experimentelle Untersuchung von kontrolliert angeregten dreidimensionalen Wellen in einer Blasius-Grenzschicht. Fortschritt-Berichte VDI, Reihe 7: Strömungstechnik 312, VDI. (in German).

Wlezien, R., D. Parekh, and T. Island (1990). Measurement of acoustic receptivity at the leading edge and porous strips. Appl. Mech. Rev. 43(5, Part 2). 


\section{Acknowledgements}

The present work would not have been possible without the guidance and support of mentors, colleges, and friends.

I am especially indebted to my committee chair Prof. Dr. D. Ronneberger and my advisor Dr. F.-R. Grosche, who initiated this study as a collaboration between the Third Physical Institute of the Georg-August University Göttingen and the Institute of Fluid Mechanics in the German Aerospace Center DLR in Göttingen. They supported my work in numerous ways, in particular, by many discussions dealing with experimental and theoretical aspects that have been decisive for the quality of this work. I also thank my co-chair Prof. Dr. H. Eckelmann for his support in many concerns and for refereeing this thesis.

I am truly thankful to Dr. Andrey V. Boiko for providing me with the linear stability solver as well as for extended discussions of the subject. Similarly, I am indebted to G. Timann and to the electronic lab of the Third Physical Institute of the Georg-August University Göttingen for the development of the DSP-system and support concerning the low-level software incorporated. I also thank D. Sturzebecher for the manufacturing of the wall-hotwire sensors and C. Gmelin for some linear stability calculations.

Many thanks to all of my colleges in the DLR as well as in the university, especially to D. Baumgarten and H.Denecke for some support concerning the experimental setup and to Dr. F. Evert, S. Hein, Dr. M. Hartmann, Dr. A. Poethke, Dr. W. Koch, Dr. G. Hoehler, Dr. H.-P. Kreplin for a lot of inspiring discussions.

The financial support of the Deutsche Forschungsgemeinschaft given as a part of the VSPP-"Transition und Turbulenz" under contract RO 369/25-(2,3) is gratefully acknowledged.

Last, but not least, I would like to thank my family and friends for their patience and understanding; a special word of appreciation goes to my fiance Svenja for her support, understanding, and encouragement. 



\section{Lebenslauf}

Zur Person

Name:

Holger Opfer

Geburtsdatum:

13. Dezember, 1970

Geburtsort:

Bad Hersfeld

Staatsangehörigkeit:

deutsch

\section{Schulbildung}

$1981-1987$

Gesamtschule Geistal in Bad Hersfeld

$1987-1990$

Modellschule Obersberg in Bad Hersfeld

Abschluss: Abitur

\section{Hochschulstudium}

1991 - 1994

1993

$1994-1995$

$1995-1998$

$1998-2002$
Physik-Studium an der Georg-August-Universität Göttingen Vordiplom

Akademisches Auslandsjahr am Trinity College Dublin

(Course: Physics)

Fortsetzung des Physik-Studiums

an der Georg-August-Universität Göttingen

Abschluss: Diplom

Thema der Diplomarbeit: "Mehrkanalige und modale aktive

Lärmkompensation in einem Modellkanal"

Aufbaustudium Physik an der Georg-August-Universität Göttingen

Göttingen, den 15.8.2002 